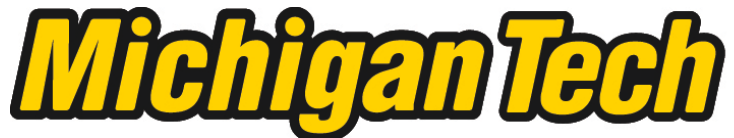 \\ Michigan Technological University Create the Future Digital Commons @ Michigan Tech
}

\section{Adapting health-risk communication to the specific cultural contexts of diverse populations : an assessment of malaria- treatment programs in Liberia}

Nathaniel Galarea Gbessagee

Michigan Technological University

Follow this and additional works at: https://digitalcommons.mtu.edu/etds

Part of the Rhetoric and Composition Commons

Copyright 2011 Nathaniel Galarea Gbessagee

\section{Recommended Citation}

Gbessagee, Nathaniel Galarea, "Adapting health-risk communication to the specific cultural contexts of diverse populations : an assessment of malaria-treatment programs in Liberia", Dissertation, Michigan Technological University, 2011.

https://doi.org/10.37099/mtu.dc.etds/85

Follow this and additional works at: https://digitalcommons.mtu.edu/etds

Part of the Rhetoric and Composition Commons 


\title{
ADAPTING HEALTH-RISK COMMUNICATION TO THE SPECIFIC CULTURAL CONTEXTS OF DIVERSE POPULATIONS: AN ASSESSMENT OF MALARIA- TREATMENT PROGRAMS IN LIBERIA
}

\author{
By \\ Nathaniel Galarea Gbessagee
}

A DISSERTATION

Submitted in partial fulfillment of the requirements for the degree of

DOCTOR OF PHILOSOPHY

(Rhetoric and Technical Communication)

MICHIGAN TECHNOLOGICAL UNIVERSITY

2011

Copyright (C) 2011 Nathaniel Galarea Gbessagee 
This dissertation, "Adapting Health-Risk Communication to the Specific Cultural Contexts of Diverse Populations: An Assessment of Malaria-Treatment Programs in Liberia," is hereby approved in partial fulfillment of the requirements for the Degree of DOCTOR OF PHILOSOPHY IN RHETORIC AND TECHNICAL COMMUNICATION.

Department of Humanities

Signatures:

Dissertation Advisor:

Committee Member:

Committee Member:

Committee Member:

Committee Member:

Department Chair:
R. Craig Waddell, Ph.D.

Marika Seigel, Ph.D.

Beatrice Smith, Ph.D.

Kurt Paterson, Ph.D.

Rima Carlson, M.D.

Ronald Strickland, Ph.D.

Date: 
I dedicate this dissertation to the memory of my son, Wydar, whose sojourn with me to the U.S. was not possible due to his untimely death in 1990, as a result of the 14year (1989-2003) civil war in my native Liberia. 


\section{Table of Contents}

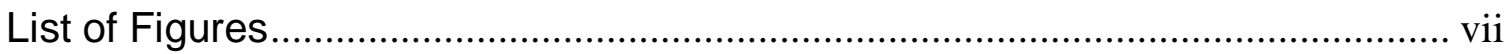

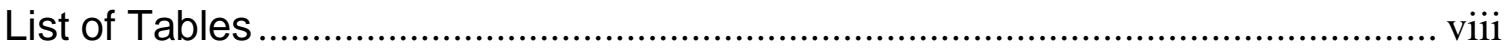

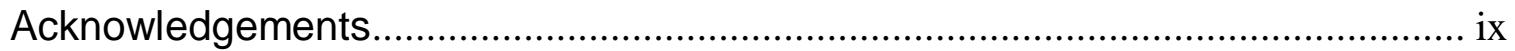

How I am Situated in this Project ..................................................................... xi

List of Abbreviations ..............................................................................................

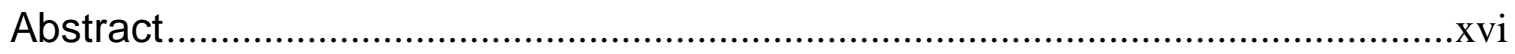

Chapter I: Risk Communication and Culture: An Overview......................................1

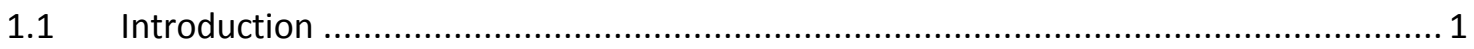

1.1.1 Definition and History of Risk (and Health-Risk) Communication ......................... 8

1.1.2 Ethnomedical Traditions in Risk Communication ........................................... 10

1.2 Why the Focus on Malaria-Treatment-and-Control Programs (MTCPs) ..................... 12

1.2.1 Malaria and Global Health Initiatives.................................................................. 15

1.2.2 Malaria Treatment and Control in Liberia.................................................... 17

1.3 Risk Communication and the Cultural Impact of Risk: A Review of the Literature ....... 23

1.3.1 Debates about Risk and Cultural Representation............................................ 23

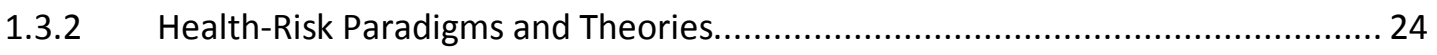

1.3.3 Worldviews as Defining Factors in Risk Communication .................................. 27

1.3.4 Effects of Health Beliefs and Culture in Risk Communication .............................. 31

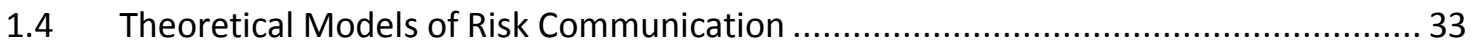

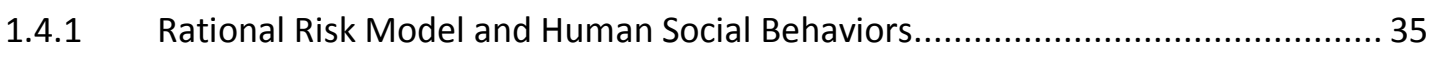

1.4.2 Uncertainty, Trust, and Credibility in Risk Communication ................................ 35

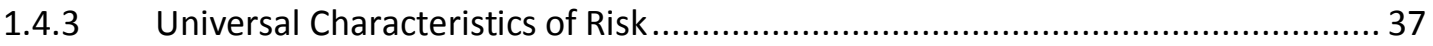


Chapter 2: Linguistic Culture and Risk Communication.

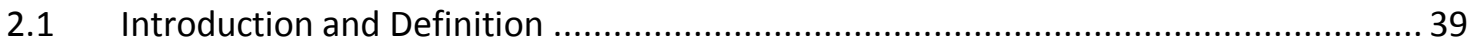

2.2 Folk Beliefs as a Consideration in Risk Communication............................................. 39

2.3 Importance of Language to Participants in Risk Communication .............................. 41

2.4 Linguistic Cultural Practices in Liberia ................................................................ 45

2.5 Diaglosia and the Territoriality of Risk Communication ............................................ 51

2.6 Dialectical Relationship of Risk Communication and Linguistic Culture .......................56

Chapter 3: Research Methods..................................................................60

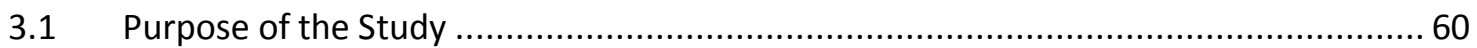

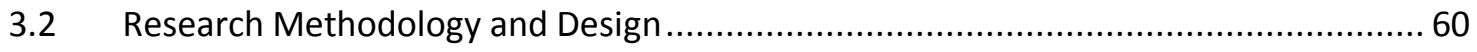

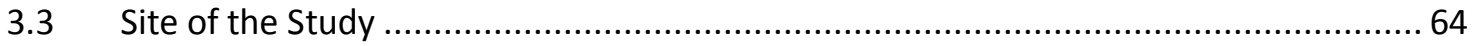

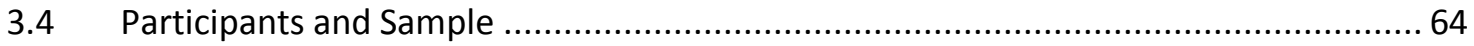

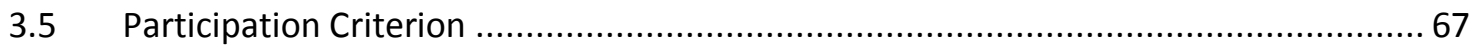

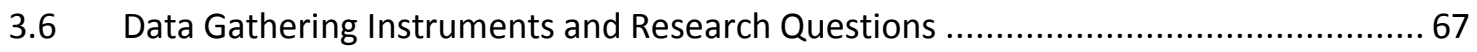

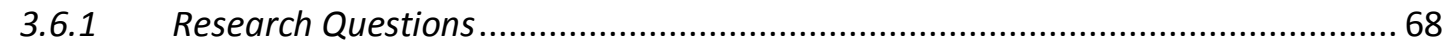

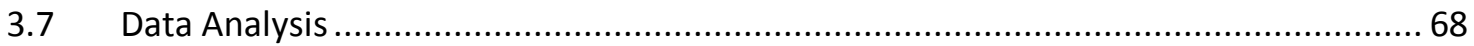

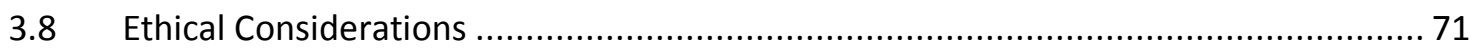

Chapter 4: PMI-Liberia and the Malaria Challenge: A Pentadic Analysis......................74

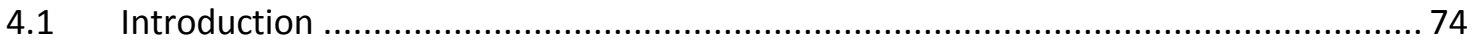

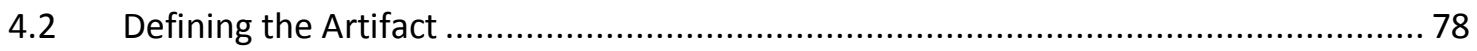

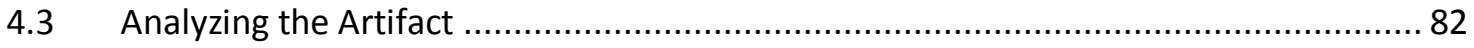

4.4 Viewing Categories of PMI-Liberia through the Pentad ....................................... 87

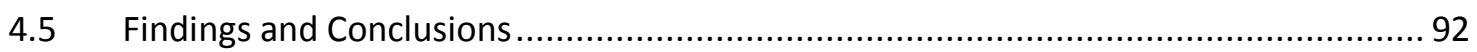

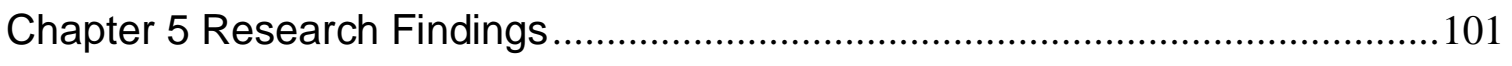

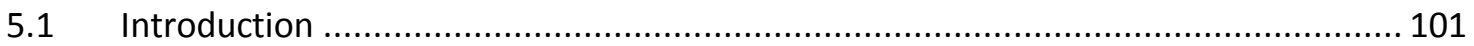

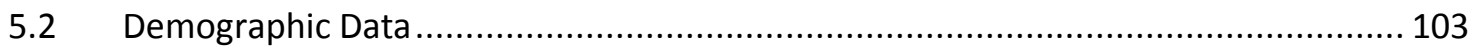

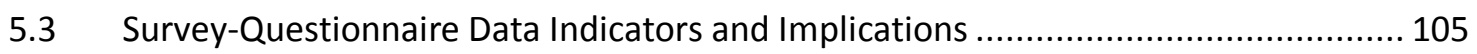

5.3.1 Respondents' Perceptions of Culture in Program Design .................................. 109

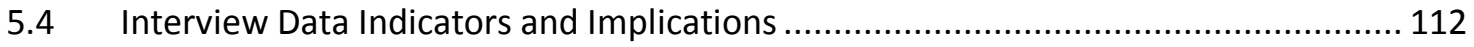


5.4.1 Participants' Knowledge and Perception of Bed Nets............................... 114

5.4.2 Participants' Perception of Language and Culture .................................... 117

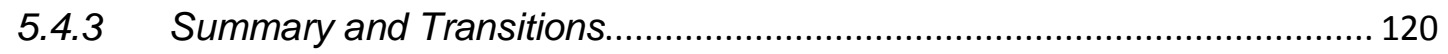

Chapter 6: Conclusions and Contributions to the Field....................................123

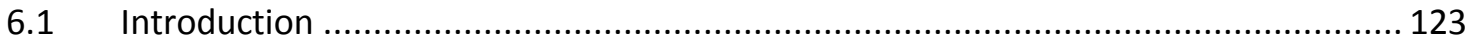

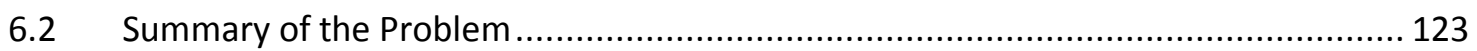

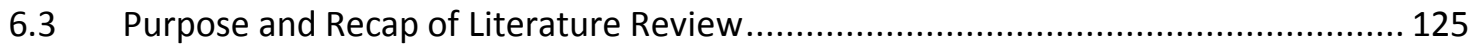

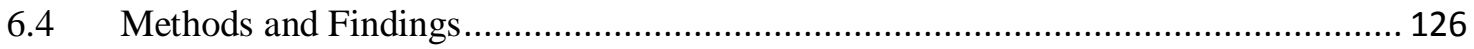

6.5 Effects of Local Culture and Traditions on Communicating Health Risks .................. 129

6.5.1 How the Design and Implementation of MTCPs Impact Malaria-Treatment

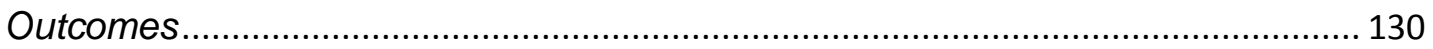

6.5.2 Funding and Other Constraints on Health-Risk Communication ............... 133

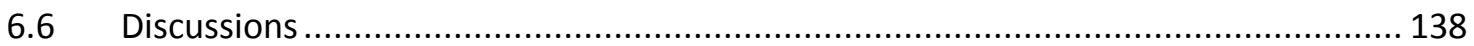

6.6.1 Dealing with Specific Cultural Contexts................................................... 143

6.6.2 Design as Rhetoric: Toward a Rhetoric of Convergence and Cultural

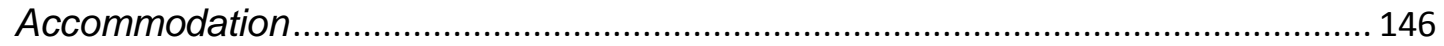

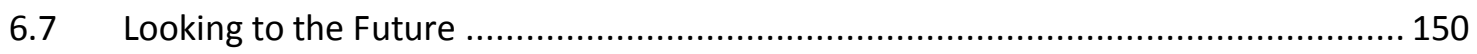

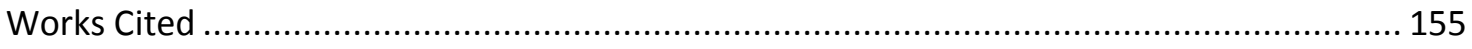

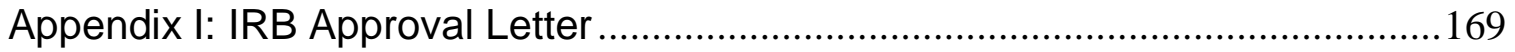

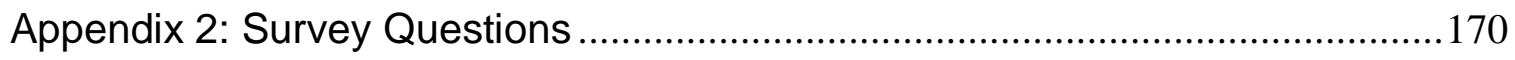

Appendix 3: Answers to Survey Questions ................................................173

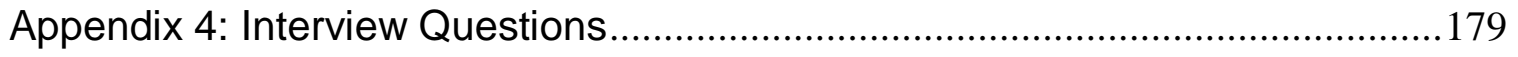

Appendix 5: Answers to Interview Questions.............................................181 


\section{List of Figures}

Figure 1.1: Regional Map of Liberia ........................................................ 6

Figure 1.2: Distribution Map of Malaria in Children in Liberia ................................ 18

Figure 2.1: Five Liberian Phonetic Scripts ...................................................... 46

Figure 2.2: New Testament Translated into Liberian Languages ............................. 47

Figure 2.3: Dialectical Graph Between Linguistic Culture and Risk Communication . 55

Figure 5.1: Ethnicity of Interview Participants ............................................. 101

Figure 5.2: Interview Participants by Community ................................................ 103

Figure 6.1: Researcher (right of briefcase) with Some Interview Participants .......... 139 


\section{List of Tables}

Table 4.1 RBM/PMI Estimates, Progress, and Target Gaps.................................... 95

Table 5.1 Respondents Discuss Cultural Inclusion in Message Design.................... 110

Table 5.2 Participants’ Perception of Bed Nets and Insecticides Use...................... 116

Table 5.3 Participants’ View of Language and Culture ........................................ 118

Table 6.1 Sample Bed Nets Distributed by NMCP Across Liberia in 2009............... 136

viii 


\section{Acknowledgements}

I want to acknowledge the invaluable contributions to my education, professional pursuits, and well-being by all persons who helped me along the way from childhood through adulthood. I want to especially thank Almighty God for giving me the wisdom, knowledge, and understanding to withstand all hardships, disappointments, and challenges placed in my way in my pursuit of higher education. I want to thank my wife, Sylvestine, and my daughters Nathlyne and Decontee, for their moral support, and my friends James N. Larsah, Isaac Saye Zawolo, Jr., Siahyonkron Nyanseor, and Bayo Omolola for timely feedback on my earlier drafts. I want to thank my dissertation adviser, Dr. Craig Waddell, for his insights, patience, and guidance in reviewing and giving me timely feedback on the many drafts encompassing the research proposal through the final dissertation.

I want to thank the staff of the National Malaria Control Program (NMCP) in Liberia, especially Communication Officer Mr. Daniel Somah and Deputy Program Director Mr. Tolbert G. Nyenswah for the invaluable assistance provided to me during my field research in Liberia. I was given ready access to the program's conference room and Internet services every time I was in Monrovia, and I had the occasion to travel to Bomi and Grand Bassa Counties with Mr. Somah and his public education and sensitization team. Several program staffers completed survey questionnaire for my research study, and I also received important supporting documents from the program to aid in my research.

My trip to Liberia would not have succeeded without the help of Mr. Moulton Kennedy, who for many weeks travelled to various offices in Monrovia to drop-off and pick-up survey questionnaires, and Mr. Kalifah Camara (KC), who doubled as driver and video cameraman during my field research in Buchanan, Grand Bassa County. I want to thank my brother Edward N. Gbessagee, Mr. Jeffery Welwean, and Mr. Solomon Murray who served as informants and tour guides for several communities across Buchanan. Mr. King also served as Bassa-language interpreter during several field interviews, as did Mr. Peter Gbater and Ms. Mamie K. Flomo. My gratitude also to 
my sister Baryo (Mrs. Edwina Baryo Hall-Mambu) for placing her car and driver (KC) at my disposal during my stay in Liberia. I owe a great debt of gratitude to all of you who have contributed so immensely to the successful completion of my research in Liberia and to my studies in general, including the late Mr. Thomas Yarjay, my private tutor in 4th grade, whose inspiration led me to pursue studies in communication and writing. 


\section{How I am Situated in this Project}

For several of my teenage years in Liberia in the late 1970s, I lived near a public landfill. No one in the community was spared the daily pungent scent and invading armies of flies, mosquitoes, and rodents. It was a miracle that the entire community was not stricken with illness, but many people did suffer constantly from malaria, severe headaches, and other fevers. It would take years before the swamp used as a dumpsite was finally filled with garbage and later covered with gravel. I was deeply concerned about my own health and about that of others in the community, but there was hardly anything anyone could do except move out. There was no public-health information or health-risk-communication action plan available to help the community cope with the effects of the dumpsite.

Later, as a practicing journalist in Liberia in the 1980s, I covered two national catastrophes that renewed my interest in health-risk-communication issues. The first catastrophe was a 1982 landslide in Liberia that resulted from a group of ruptured tailing dams at a mining facility. Mineral wastes from the tailing dams overran makeshift dwellings for miners in the adjacent valleys, and scores of miners were buried alive. The second catastrophe was an Air Liberia plane crash during an emergency landing in Khartoum, Sudan in 1983. Seven of the plane's eight occupants, mostly pilots and crewmen, were burnt to death after the plane ran into high-voltage electrical wires near the airport, crashed into a house, and caught fire. Indeed, as I walked through hospital morgues in Liberia and Sudan to report on the landslide and plane crash, and as I visited the original scenes of both incidents, I couldn’t help but imagine the lives that could have been saved in either case had there been a robust health-risk-communication system in place. The miners could have been persuaded not to construct makeshift dwellings in valleys near the tailing dams, and steps could have been taken to monitor the tailing dams for possible overflow. And the airport in Khartoum could have prevented low-hanging, high-voltage electrical wires and private dwellings from being constructed nearby, which would have avoided the catastrophe that befell the Air Liberia plane in what would have otherwise been a safe emergency landing. These 
incidents and a two-week, international-science-and-technology-reporting workshop I attended in Nairobi, Kenya in 1984 drew me to health-risk communication. The workshop — which included field trips to the Kenyan rift valley, the Nairobi National Museum, and the Kenyan Institute of Tropical and Infectious Diseases-focused on strategies for communicating health risks about the ozone layer, climate change, malaria, tuberculosis, African trypanosomiasis, and related health and environmental problems.

Moreover, as late as 2005, my colleagues and I in the U.S.-based Liberian History, Education, and Development, Inc. (LIHEDE) held a symposium on combating malaria in post-conflict Liberia. The symposium brought together health experts from USAID, the CDC, Malaria International, the Liberian Malaria Control Program, and other agencies to find ways to curb the high incidence of malaria in Liberia. Liberia is home to one of the deadliest strains of malaria—plasmodium falciparum — and a 14year civil war (1989-2003) shattered the country's healthcare-delivery system. Based on Liberia's health statistics, malaria now accounts for most in-patient and out-patient hospital visits and is the leading cause of deaths among infants, pregnant women, and adults. Therefore, the goal of the symposium was to rally international support to combat malaria in Liberia. The resolution from the symposium was shared with U.S. and Liberian government authorities and with international aid organizations. This was followed by a letter-writing campaign that resulted in Liberia becoming a recipientcountry of the President's Malaria Initiative (PMI), a U.S.-government aid program for fighting malaria in sub-Saharan Africa. As co-founder and secretary-general of LIHEDE, I contributed my time and resources to making the symposium and letterwriting campaign successful. These activities reinforced my interest in health-riskcommunication issues.

As a result, when I entered the PhD Program in Rhetoric and Technical Communication at Michigan Technological University, I decided to pursue risk communication and cultural studies as minor areas of study (composition being my major area of study) to get a better understanding of the cultural importance of the health-risk issues to which I have been exposed over the years. I took a risk 
communication course with Dr. Craig Waddell in 2008, and I did a year of directed readings on health-risk communication and rhetoric with Dr. Waddell in 2009 and 2010, as part of preparations for writing my dissertation proposal and dissertation. I also audited Dr. Waddell's classical rhetoric class in 2010, and in the summer of 2011, I taught an elective course in risk communication for undergraduate seniors and graduate students. Dr. Waddell later agreed to chair my dissertation committee, and I feel fortunate to have benefitted from his knowledge and expertise in structuring, researching, refining, and writing this dissertation.

In addition to my studies in risk communication and rhetoric, I did a graduate course in humanistic qualitative research methods with Dr. Patricia Sotirin in 2007, which helped me to prepare for and undertake a field study of malaria-treatment-andcontrol programs (MTCPs) in Liberia for this dissertation. Specifically, I drew on risk communication and rhetorical strategies_-such as Pascal's Wager, One and Two-Way Jeffersonian approaches, community involvement, expert disagreement, and degree of uncertainty - to assess how advocates of bed-net use have adapted their messages and programs to local populations and their cultures.

I undertook this research in order to engender better understanding of how the enormous socioeconomic burdens and strains that malaria places on societies could be mitigated. I not only looked at the impact of culture and traditions on communicating health-risk information, but also at constraints that have the potential to impede the success of any health-risk-communication initiatives, including inadequately trained personnel, funding, cultural resistance, corruption, and governmental bureaucracy. I believe that the healthcare-delivery systems in Liberia could be greatly improved if the design and implementation of health-risk programs were adapted to the culture and traditions of local populations. 


\section{List of Abbreviations}

\begin{tabular}{|c|c|}
\hline ACT & Artemisinin-based Combination Therapy \\
\hline $\mathrm{AQ}$ & Amodiaquine \\
\hline AS & Artesunate \\
\hline BCC & Behavior Change Communication \\
\hline CDC & Centers for Disease Control and Prevention \\
\hline DDT & Dichlorodiphenyltrichloroethane \\
\hline DHS & Demographic and Health Survey \\
\hline FBOs & Faith-based Organizations \\
\hline GFATM or Global Fund & The Global Fund for Aids, Tuberculosis, and Malaria \\
\hline GHI & Global Health Initiative \\
\hline GHR & Global Health Risks \\
\hline GOL & Government of Liberia \\
\hline HMIS & Health Management Information System \\
\hline IEC & Information, Education and Communication \\
\hline IPTp & Intermittent Preventive Treatment of Pregnant Women \\
\hline IRS & Indoor Residual Spraying \\
\hline ITN & Insecticide-treated mosquito net \\
\hline LDHS & Liberia Demographic and Health Survey \\
\hline LIHEDE & Liberian History, Education, and Development, Inc. \\
\hline LLIN & Long-Lasting Insecticide-Treated Bed Net \\
\hline LMIS & Liberia Malaria Indicator Survey \\
\hline M\&E & Monitoring \& Evaluation \\
\hline MCMOM & Malaria Case Management Operation Manual \\
\hline MSF & Médecins Sans Frontières or Doctors Without Borders \\
\hline MIP & Malaria in Pregnancy \\
\hline MOH\&SW & Ministry of Health and Social Welfare \\
\hline NMSP & National Malaria Strategic Plan \\
\hline
\end{tabular}


MTCPs

NDCL

NDS

NGOs

NMCP

PMI

$\mathrm{RBM}$

RDT

UNDP

UNICEF

USAID

WHO
Malaria Treatment and Control Programs

National Traditional Council of Liberia

National Drug Service

Non-Governmental Organizations

National Malaria Control Program

The U.S. President's Malaria Initiative

Roll Back Malaria

Rapid diagnostic test

United Nations Development Program

United Nations Children’s Emergency Fund

United States Agency for International Development

World Health Organization 


\section{Abstract}

Drawing on theories of technical communication, rhetoric, literacy, language and culture, and medical anthropology, this dissertation explores how local culture and traditions can be incorporated into health-risk-communication-program design and implementation, including the design and dissemination of health-risk messages. In a modern world with increasing global economic partnerships, mounting health and environmental risks, and cross-cultural collaborations, those who interact with people of different cultures have "a moral obligation to take those cultures seriously, including their social organization and values" (Hahn and Inhorn 10). Paradoxically, at the same time as we must carefully adapt health, safety, and environmental-risk messages to diverse cultures and populations, we must also recognize the increasing extent to which we are all becoming part of one, vast, interrelated global village. This, too, has a significant impact on the ways in which healthcare plans should be designed, communicated, and implemented.

Because communicating across diverse cultures requires a system for "bridging the gap between individual differences and negotiating individual realities” (Kim and Gudykunst 50), both administrators and beneficiaries of malaria-treatment-and-control programs (MTCPs) in Liberia were targeted to participate in this study. A total of 105 people participated in this study: 21 MTCP administrators (including designers and implementers) completed survey questionnaires on program design, implementation, and outcomes; and 84 MTCP beneficiaries (e.g., traditional leaders and young adults) were interviewed about their knowledge of malaria and methods for communicating health risks in their tribe or culture. All participants showed a tremendous sense of courage, commitment, resilience, and pragmatism, especially in light of the fact that many of them live and work under dire socioeconomic conditions (e.g., no electricity and poor communication networks). 
Although many MTCP beneficiaries interviewed for this study had bed nets in their homes, a majority (46.34 percent) used a combination of traditional herbal medicine and Western medicine to treat malaria. MTCP administrators who participated in this study rated the impacts of their programs on reducing malaria in Liberia as moderately successful (61.90 percent) or greatly successful (38.10 percent), and they offered a variety of insights on what they might do differently in the future to incorporate local culture and traditions into program design and implementation. Participating MTCP administrators and beneficiaries differed in their understanding of what “cultural incorporation” meant, but they agreed that using local indigenous languages to communicate health-risk messages was essential for effective health-risk communication. They also suggested that understanding the literacy practices and linguistic cultures of the local people is essential to communicating health risks across diverse cultures and populations. 


\section{Chapter I: Risk Communication and Culture: An Overview}

\subsection{Introduction}

In Risk, cultural studies professor Deborah Lupton talks about two dominant but complementary perspectives underpinning theories and practices in risk communication research: the cognitive science perspective and the sociocultural perspectives, which undergird the focus of this dissertation. Whereas proponents of the cognitive science perspective tend to look at risk by means of probability calculations based on the notion of risk as "the product of the probability and consequences (magnitude and severity) of an adverse event” (qtd. in Lupton 17-18), the proponents of sociocultural perspectives tend to believe that risk is socially and culturally constructed within specific contexts in such a way that it must be negotiated in order to be understood.

Sociocultural proponents also contend that "a risk is never fully objective or knowable outside of belief systems and moral positions: [because] what we measure, identify and manage as risks are always constituted via pre-existing knowledges and discourses” (Lupton 29). Nevertheless, Lupton indicates that in contemporary Western societies, 'the noun 'risk' and the adjective 'risky' have become very commonly used in both popular and expert discourses... [as a result of which] An apparatus of expert research, knowledge, and advice has developed around the concept of risk: risk analysis, risk assessment, risk communication, and risk management” (9). Therefore, risk communication and associated fields of research and practice, such as risk analysis and risk management, are generally "used to measure and control risk in areas as farreaching as medicine and public health, finance, the law, and business and industry” (Lupton 9).

Proponents of the cognitive science perspective use the psychometric paradigm as the main methodological approach, while proponents of the sociocultural perspectives use a combination of epistemological and methodological approaches drawn from structuralism, post structuralism, phenomenology, and psychoanalysis (Lupton 24-28). According to Lupton, the sociocultural perspectives encompass such other perspectives as the "cultural/symbolic" perspective led by Mary Douglas; the 
"risk society" theorists led by Ulrich Beck and Anthony Giddens; and the "governmentality theorists" who based their work on the writings of Michael Foucault (24). In Section 1.3.2 of this chapter, I discuss approaches affiliated with the cognitive science and sociocultural perspectives, and in chapter 2, Section 2.5, I discuss the governmentality approach.

Because language, culture, and literacy (either acting together or alone) canand do-play a crucial in how well we communicate across cultures , I increasingly find myself more situated within the realm of the sociocultural perspective than the cognitive science perspective. Mohan Dutta’s culture-centered approach not only makes culture the foundation of any form of human communication, including risk communication, but Lundgren and McMakin have also noted that "risk communication becomes more complicated when it crosses borders” (332) due to factors such as language and culture and “competing regulations and political climates” (333).

The influences of language and culture explain why human perceptions of risk (psychology); human cultural patterns, ways of life, and worldviews (anthropology/cultural studies); and human modes of communication and communicative practices and choices (communication) have become important considerations in the design of risk-communication messages throughout the modern world. For instance, Witte, Meyer, and Martell argue that because health-risk messages usually focus on helping people think about both potential and actual threats to their health and wellbeing, the "job [of risk communicators] should be to induce certain perceptions, because perceptions (or thoughts) are the basis of [every human] action” (20). Given this behavioral-change perspective, Thomas Abraham suggests that the primary goal of risk communicators, especially health-risk communicators, should be to determine how best "to transmit health [or risk] information clearly to stakeholders in ways that encourage behavioral changes to reduce the risk" (3).

Yet rapid developments in communication technologies (e.g., radio, cell phone, computer, Internet, and cable television) have not made human communication much easier for everyone. Many people still have difficulties communicating across cultures and with one another due to language barriers, cultural and religious practices, and 
general socioeconomic conditions. And, as the World Health Organization (WHO) suggests, mitigating local and global health risks in this 21st century demands "a shared responsibility, involving equitable access to essential care and collective defense against transnational threats" such as malaria, HIV/AIDs, and tuberculosis (GHR 6). Hence, communicating health risks across diverse cultures and populations calls for close global collaboration and understanding of the local cultures and traditions of individual nations and communities.

In this dissertation, I analyze Malaria-Treatment-and-Control Programs (MTCPs) in Liberia as part of a broader effort at understanding how best to adapt health-risk communication to the specific cultural contexts of diverse populations. I do this by reviewing policy documents (e.g., organizational plans, manuals, and annual reports) of selected MTCPs—and by interviewing MTCP beneficiaries and conducting a survey involving MTCP administrators - to determine the appropriate level of cultural adaptation for overall program design and implementation. Because my goal is to find out - if any - the extent to which local culture and traditions can be incorporated into program design and implementation, I approximate an appropriate level of cultural adaptation to mean the tailoring of health-risk messages and related MTCPs to the cultural and linguistic concerns of people at the national, ethnic group, or community level. Hence, while I use the word local (i.e., "local culture and traditions") to refer mainly to the cultural and linguistic practices of people of the indigenous segments of Liberian society who neither speak nor read and write English—English is the official language and medium of instruction in Liberia-I also contemplate that local will be understood to encompass the way of life of people in a country or geographic region. By defining "local” so broadly, I contemplate that both cultural literacy and language fluency will impact the way in which health-risk messages are designed and communicated within individual societies and across diverse cultures and populations. 
One of key insights that emerges from the sociocultural perspectives is how best to identify ways in which "underlying cultural structures, hierarchies and categories serve to define risk knowledges and practices” within a given society or community (Lupton 26). Mary Douglas argues that each culture is not only "being self-defined by opposition to the others," but also that "any community has several cultures, and that each culture defines itself by contrast with the others” to an extent that persons who share a culture often "maintain enthusiasm for it by charging the other cultures with moral failure” (7). This sort of enthusiasm for individual cultural values imposes a great challenge that demands tact and care in how we communicate with people from different cultures.

In undertaking a study to find out how and to what extent local cultures and traditions are incorporated into the design and implementation of MTCPs and healthrisk messages in Liberia, I endeavor to focus attention on a crucial-but often neglected-aspect of risk-communication research and practice that is germane to the success of current global efforts at effective malaria treatment and control. For example, in their comparative study of Cultural Theory and the psychometric paradigm, Marris, Langford, and O'Riordan lament that "Most risk perception studies have focused too much on 'the public' in the aggregate and not enough on differences between individuals and groups, analyzed within specific social and institutional contexts” (646). They argue that because risk-communication strategies formulated through this approach are "not necessarily adapted to the demands of different social groups," a need exists for developing new sets of risk-communication strategies "with both individualistic and cultural associations in mind” (646).

This appeal to individualistic and cultural perspectives in risk-communication research and practice by Marris, Langford, and O'Riordan aligns forcefully with the goal of this study to prioritize the "local" in the process of designing and implementing, MTCPs and designing and communicating health-risk messages in Liberia. For example, in his theory of intentionality, John Searle hypothesizes two types of background: deep background and local background, which he says influence intentionality. He associates biological skills and related human activities (e.g., eating, 
talking, and walking) with deep background and cultural skills (e.g., the ability to recognize specific cultural objects, situations, and behaviors) as local background (143144). In this context, deep background, local background, and local cultures and traditions exert tremendous influence not only on intentions, but also on how health-risk messages and programs are designed, implemented, and communicated.

Thus, if the goal of risk communicators is to use risk information to forestall or correct the spread of hazardous situations and materials and related health, safety, and environmental risks, then health-risk messages must be tailored to and disseminated among audiences based on the local cultures and traditions of these audiences. And because many nations of the modern world are multicultural, multilingual, multiethnic, and multi-religious entities, almost any risk-communication situation involves peoples of diverse backgrounds and cultures. Every human action-including individual dispositions toward risk or risk information-is influenced by culture in the sense that almost all human beliefs and social practices are byproducts of the culture to which each individual belongs.

In this first chapter, I explore the interconnections between risk communication and culture using MTCPs in Liberia as background. Liberia (see Figure 1.1) is a hotbed for malaria infestation due to its tropical rainforests. The country's healthcare system is also still recovering from the devastating effects of a 14-year civil war (1989-2003). Malaria kills millions of people annually, and information about its treatment and control could save so many lives. I also explore how risk communication and culture tie into the greater issues of ethno-medicine, global-health risks, and creation of health-risk messages across diverse cultural populations.

This chapter is subdivided into four sections: Sections 1.1 and 1.2 provide the background and rationale for the dissertation, and Sections 1.3 and 1.4 provide a review of relevant literature. The first two sections provide an overview of the definition and history of risk communication, ethnomedicine, global health risks, and the state of malaria control in Liberia. The last two sections provide a survey of current riskcommunication practices and theories and ongoing debates within the field, such as uncertainty, trust, credibility, health beliefs, and worldviews. 


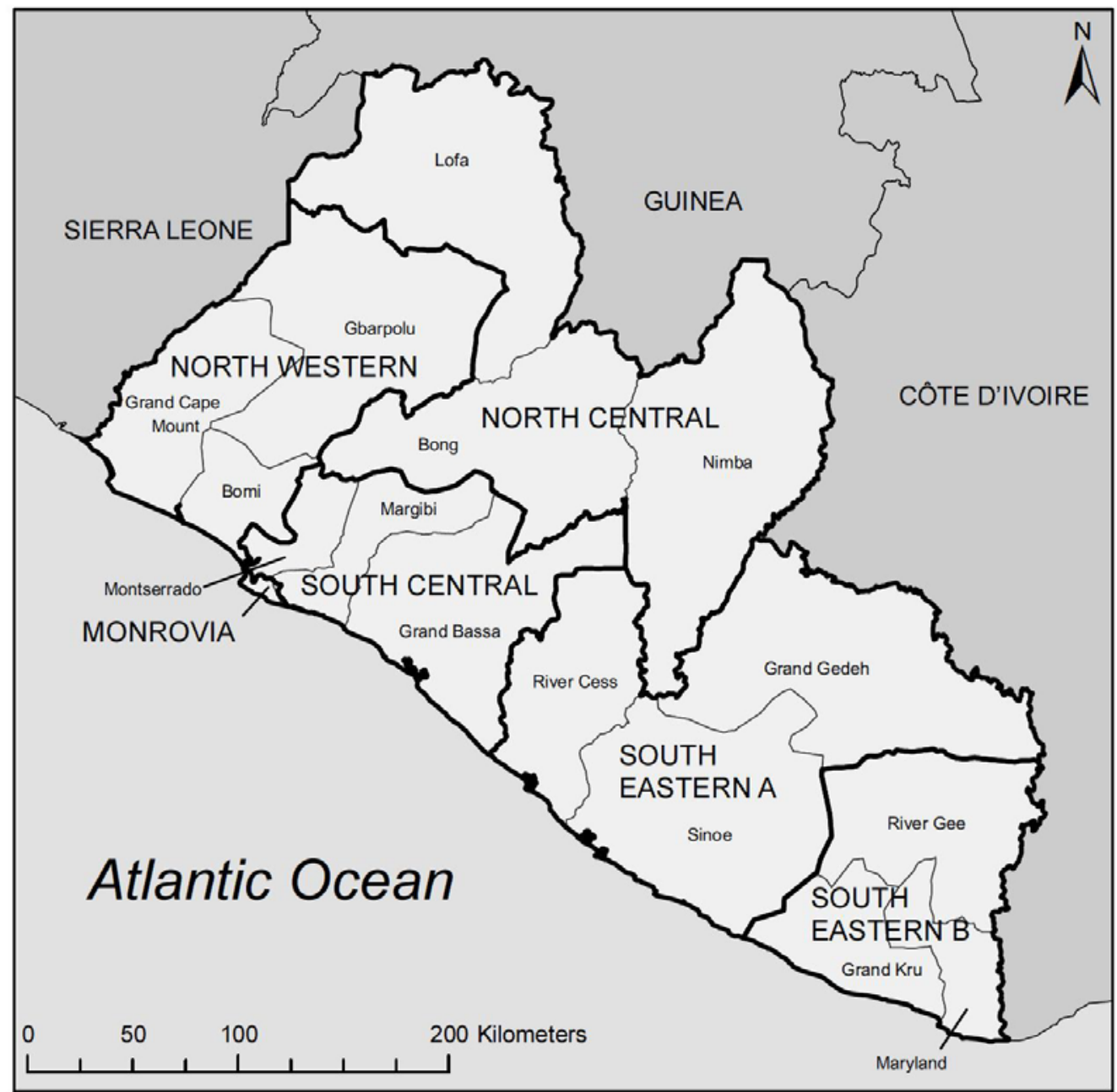

Figure 1.1: Regional Map of Liberia

In Chapter 2, "Linguistic Culture and Risk Communication,” I rely on Harold Schiffman's definition of linguistic culture to argue that language and culture are not only invaluable tools for constructing effective health-risk messages across cultures, but they also dictate how people within each society perceive and react to risks. The chapter further explores factors underpinning the collaborative use and application of language and culture in risk communication, including folk beliefs, linguistic practices, and language development.

In Chapter 3, "Research Methods,” I focus on the methodology and analytic strategies used for collecting and analyzing the research data. These strategies provide the contexts for participant selection and data collection and analysis; they also provide rationale for using rhetorical devices_rather than, for example, grounded theory-to 
analyze data collected through qualitative methods. While the traditional grounded theory, ethnography, and phenomenology are widely used with most qualitative studies such as this one, I opted to use analytic rhetorical tools such as pentadic criticism and generative criticism to analyze the research data because I wanted to emphasize the rhetorical appeals embedded in health-risk message design and human communication in general. In Chapter 4, "PMI-Liberia and the Malaria Challenge: A Pentadic Analysis,” I use pentadic criticism to analyze the 2008 President's Malaria Initiative (PMI)'s Malaria Operational Plan (MOP) for Liberia. PMI is one of the main pillars of malaria-treatment-and-control efforts in Liberia.

In Chapter 5, "Research Findings," I discuss the results of my field research in Liberia. In Chapter 6, “Conclusions and Contributions to the Field,” I provide a summary of the problem statement, recap the insights gleaned from the literature review, and explore and explain the findings and conclusions that emerged during this study. I conclude the chapter with recommendations for future study. 


\subsubsection{Definition and History of Risk (and Health-Risk) Communication}

The U.S. National Research Council (NRC) defines risk communication as "the interactive process of exchange of information and opinions among individuals, groups, and institutions concerning a risk or potential risk to human health or the environment” (NRC 1989). And the World Health Organization (WHO) defines health risk as "a factor that raises the probability of adverse health outcomes” (GHR 6). Accordingly, health-risk communication is a subset of risk communication that deals exclusively with the creation and dissemination of technical, informational, and educational messages that address specific health-risk problems and issues, such as malaria, tuberculosis, HIV/AIDS, and diarrhea. Throughout this dissertation, the terms "risk communication" and "health-risk communication" are used interchangeably in order to elaborate key concepts and theories about health and illness, with the goal of engendering understanding of how best to adapt health-risk communication to the specific cultural contexts of diverse populations.

Plough and Krimsky suggest that "although the study of risk communication [in 1986 was] considered new, the practice of it may be as old as human culture itself” (224). They argue that "public risk perceptions and individual behavioral responses to risks” (224) predate widespread discussions in the 1980s about risk communication as a theoretical framework in public policy in the areas of health and the environment (223). On the other hand, Professor Craig Waddell suggests that although "as a profession and a subject of scholarly discussion, risk communication is a fairly new field, the practice of communicating hazards is ancient, diverse, and ubiquitous, including everything from fairy tales to road signs” (“Defining” 207). Together, Plough, Krimsky, and Waddell point to a practice of identifying and attempting to control various categories of risks within individual cultures, which underpins the new but growing academic discipline of risk communication. Consistent with this view, Lundgren and McMakin indicate that since the 1980s, risk communication has evolved into a full-fledged academic discipline that "encompasses many types of messages and processes” dealing with human health, safety, and the environment (5). 
Because risk communication involves "multiple messages about the nature of risk and other messages, not strictly about risk, that express concerns, opinions, or reactions to risk messages" (NRC), it "can help people with differing perspectives and levels of expertise to share a common understanding of the level of risk (actual danger) involved in a particular activity” (Beecher et al. 2002). Beecher et al. also note that while risk communication seeks either to decrease or to increase the level of public concerns and perception about particular risk events, it should never substitute for risk management nor should it seek to hide something or to manipulate opinions (125): "Rather, its aim is to ensure that a diverse range of people share a common, accurate understanding of the level of [a particular] risk" (Beecher et al. 125). Lundgren and McMakin add that "The process of risk communication begins with a hazard, a potential or actual danger to the environment or human health or safety” (5), which they say mandates active community involvement and collaboration at every stage in containing health risks of any kind. Risk communication is (or should be) a participatory process that involves "people in all walks of life-parents, children, legislative representatives, regulators, scientists, farmers, industrialists, factory workers, and writers” (Lundgren and McMakin 5) as well as traditional chiefs, elders, and people in both rural and urban communities.

This participatory process also demands language proficiency—i.e., proficiency in the languages that are widely spoken in a given society—as a key communicative factor in adapting health-risk communication to the specific cultural contexts of diverse populations. As Schiffman suggests, "language is the primary vehicle of acculturation, of learning one's culture” (58). Hence, language is an indispensable tool of human communication in both traditional and non-traditional societies and cultures in that almost all person-to-person communication takes place through language. This concept of language as the quintessential tool of acculturation and communication-i.e., a society's "linguistic culture” (Schiffman 2004)—is explained in detail in Chapter 2. 
Since "health risks seem to loom around every corner, posing a constant threat to the public” (Conrad 422), the processes of creating and disseminating health-risk messages ought to be informed by the cultural perceptions of health and illness in each society. From a cultural perspective, Jan Nederveen Pieterse argues that in the modern world, "There are no fixed boundaries other than those of common social experience, therefore there are no territorial limitations to culture” (42). Pieterse also argues that "while borders and boundaries are a function of differentials of power, they are social constructions that are embedded and encoded in cultural claims” (117). Thus, Pieterse points to a new global reality in which interactive cross-cultural human relations and cooperation make communicating health risks to a diverse population a complex but challenging undertaking.

\subsubsection{Ethnomedical Traditions in Risk Communication}

Virtually all human behaviors and reactions are shaped by culture. People’s attitudes, actions, beliefs, and sensibilities about health and illness are embedded within specific cultural contexts and systems that differ from society to society, ethnic group to ethnic group, and individual to individual. This is why "the same 'disease' (such as tuberculosis) or symptom (such as pain) may be interpreted completely differently by two individuals from different cultures or social backgrounds and in different contexts” (Helman 83). Moreover, according to Huff and Kline, "Westerners often consider biomedicine the only reliable health resource, but people around the world-and many in the United States—use other healing systems as well” (164).

While no one can honestly undervalue the contributions of biomedicine toward sustaining many modern health systems around the globe, it is even more difficult for anyone to underrate the influence of lay theories and traditional practices of health and illness (i.e., alternative medicine, including herbal treatment and spiritual healing) in many developing nations of the world. This explains why in many Western societies, the onus for illness is usually placed on individual actions, while in most non-Western societies, the onus for illness is often placed on social actions attributed to "witchcraft, sorcery and the 'evil eye’” (Helman 93). Hence, in almost all human societies and 
cultures, "competing ethnomedical traditions coexist and form distinct health subcultures with unique beliefs, practices and organizations” (Huff and Kline 164).

These pluralistic ethnomedical systems — which may include "midwives, spiritual healers, diviners, faith healers, herbalists, root doctors, many others labeled as involved in magical, religious, or superstitious practices” (Huff and Kline 176-177) are found in every modern society, including the United States and Liberia, and they play pivotal roles in how health and related risks are defined, communicated, and handled. This is why many scholars in risk communication, medical anthropology, and global-health policy have tended to agree that how people perceive, communicate, and treat risks (i.e., health, safety, or environmental) depends largely on their cultural orientation and local socioeconomic conditions. Huff and Kline claim that "a particular population or targeted group will choose to participate in health promotion and disease prevention programs” due mainly to cultural considerations (3). Hence, individual and societal perceptions of risk, health, and illness vary greatly, and the ability of individuals to acknowledge and respect other people's cultures—as well as to learn how to interact with people of different cultures—is vital to the success of communicating health-risk to diverse populations and cultures. This is why in differentiating between perceptions of health and wellness in Western and non-Western societies, Helman says that attributing illness to witchcraft is "more common at times of social change, uncertainty and social conflict” (93). It is exactly these sorts of uncertainties in postconflict Liberia that necessitate the focus of this dissertation on the cultural aspects of MTCPs.

As a country still recovering from the residual effects of a devastating14-year (1989-2003) civil war, Liberia is an ideal environment for all sorts of uncertainties. However, these uncertainties are not about witchcraft and other forms of misfortunes; instead, they are about whether health programs designed and implemented by international NGOs and other aid agencies in Liberia to treat, prevent, or eradicate malaria can succeed without being adapted to the local culture and traditions. In this respect, two categories of MTCPs in Liberia are analyzed and complimented by ethnographic field research as part of a broader effort at understanding how to adapt 
health-risk communication to the specific cultural contexts of diverse populations: (1) ongoing or recently completed MTCPs developed on a Western model and implemented in Liberia pretty much "off the shelf,” and (2) those adapted, for instance, to a particular audience, such as a Liberian ethnic group, community, village, or extended family.

\subsection{Why the Focus on Malaria-Treatment-and-Control Programs (MTCPs)}

The focus on MTCPs rather than on other treatment-and-control programs, such as those on HIV/AIDS and polio, underscores the lingering effects of malaria as a curable but deadly disease that has afflicted humans for millennia. In 1880, French military surgeon Charles Louis Alphonse Laveran discovered the malaria parasite during laboratory trials after the disease had afflicted and killed hundreds of French troops (Malaria Site). According to Michael Finkel, only few civilizations in history have survived the ravages of malaria. Finkel reports that “A million Union Army casualties in the U.S. Civil War are attributed to malaria, and in the Pacific theater of World War II casualties from the disease exceeded those from combat" (Finkel 3). He also reports that malaria killed four popes and severely afflicted three U.S. presidents: George Washington, Abraham Lincoln, and Ulysses S. Grant (3). Finkel also claims that the death of Alexander the Great from malaria led to disintegration of the Greek (3).

Today, malaria continues to afflict millions of people worldwide, mostly in Africa, Asia, and the Middle East. As a result, strategic global-health initiatives—such as the U.S. President's Malaria Initiative (PMI), Rollback Malaria Partnership (RMP), and The Global Fund to Fight AIDS, Tuberculosis and Malaria (Global Fund)-have emerged as leading institutions and programs working to combat the global spread and menacing effects of malaria. Yet in spite of billions of dollars spent annually on the design and implementation of these initiatives, the WHO has acknowledged that malaria remains a major, world-wide killer. There are currently no vaccines on the commercial market against malaria. However, in October 2011, the results from clinical trials of 
phase III of the RTS, S vaccine among 15,460 children (ages 5 and 7 months) from Kenya, Burkina Faso, Gabon, Ghana, Malawi, Mozambique, and Tanzania found a 53 percent and a 65 percent reduction in the risk of malaria infection among children over eight months and over six months, respectively (Dathura). I n December 2011, the global malaria death rate fell from malaria killing a child every 30 seconds (2007 estimates) to every 45 seconds (2009 estimates) to every 60 seconds in 2011 (BBC). These new estimates and clinical trials hold up some promise in combatting malaria worldwide, but they have yet to compensate for the billions of dollars spent over the years on malaria treatment and control. A combination of indoor residual spraying, antimalarial drugs, and insecticide-treated mosquito nets (also known as bed nets) has become the mainstay of current global efforts at malaria treatment, control, prevention, and eradication, but these efforts have failed to eradicate malaria. According to a U.S. Pentagon official, in spite of taking antimalarial drugs, 80 of 200 U.S. troops who entered Liberia in 2003 during the Liberian civil war to secure the national airport and seaport in support of the West African Peace Keeping Force (ECOMOG) contracted malaria (NZ Herald).

The missing factor in all of these efforts is the extent to which the culture and traditions of local communities and townships across the world have been incorporated into the design and implementation of MTCPs. Hence, in addition to assessing MTCPs in Liberia to find out what effects local Liberian culture and traditions have on the process of communicating health risks to affected populations, I have reviewed current literature on health-risk communication and cultural adaptation theories in an effort to determine the underlying cultural and social factors that promote or hinder effective health-risk communication across cultures and populations. Because different MTCPs may report their results differently, in comparing program outcomes, a common standard of measurement (such as reduction in deaths from malaria per capita over the year following program implementation) was established. In addition, possible inaccuracies in program reports about goals and levels of cultural adaptation were evaluated, and attempts were made to control for confounding variables, such as dollars invested per person served and appeals to celebrity status. 
Each nation, culture, or ethnic group has unique "characteristics that affect how people perceive and communicate risks” (Lundgren and McMakin 330); hence, local cultural "perceptions, interests, and ways of life” (Hahn and Inhorn 10) ought to be key determining factors in designing and implementing any local, collaborative, health-andwellness schemes and global health initiatives. Assessing MTCPs in Liberia leads to further inquiries and discussions about global health, the role of NGOs and other aid agencies in designing and implementing global health policy, and the level of cooperation between these international entities and local governments and people.

This research suggests further questions, such as, How might cultural adaptation affect the outcomes of MTCPs that adapt health-risk communication to a particular culture or ethnic group? How do constraints—such as lack of adequately trained staff, limited funding, cultural resistance, corruption, and governmental bureaucracy-impact health-risk communication in Liberia? and How might MTCPs designed and implemented by international NGOs and aid agencies succeed without adaptation to the local culture and traditions of a host country?

Drawing on these cultural insights, this dissertation critically examines the design and implementation of selected MTCPs in Liberia that are funded and/or managed by international NGOs and other aid agencies. This will determine what effects local culture and traditions have on the process of communicating health risks and how health-risk communication can be adapted to the specific cultural contexts of diverse populations. Paradoxically, at the same time as we must carefully adapt healthrisk communication to diverse cultures, we must also recognize the increasing extent to which we are all becoming part of one, vast, interrelated global village. This, too, has a significant impact on the ways in which healthcare plans should be designed, communicated, and implemented. Clearly, modern humans live not only "in the midst of rapid cultural change and increasing intercultural connectedness” but also in the midst of "impressive development in communications and transportation technology [that] moves us closer to the vision of a 'global village’” (Kim and Gudykunst 299). Understanding the diversity and interconnectedness of peoples and cultures of the world 
as participants in a vast global cultural village provides the impetus for adapting healthrisk communication to specific cultural contexts of diverse populations.

\subsubsection{Malaria and Global Health Initiatives}

In the 2009 publication Global Health Risks (GHR), the WHO identifies malaria, AIDS, and tuberculosis as three transnational diseases and threats to human health whose treatment, control, and eradication require shared international responsibility and commitment. GHR defines health risk as any factor that "raises the probability of adverse health outcomes" (6), and it defines global health as the "goal of improving health and achieving equity in health for all people worldwide” (GHR 6). Accordingly, malaria is a global health risk of endemic proportions. More than 40 percent of the world's population—about 2.5 billion people in over 90 countries (MFI 1997) — is at risk of contracting malaria each year, and malaria causes the deaths of between one and three million people each year worldwide, including 850,000 children under five years of age (GHC Fact Sheet). Malaria also accounts for 10 to 30 percent of all hospital admissions worldwide each year (Helman 255). Hence, "malaria is arguably the most important disease in the world with an estimated 350-500 million clinical cases each year" (Asase and Oppong-Mensah 493).

In sub-Saharan Africa, malaria accounts for about " $40 \%$ of public health expenditure, $30 \%$ to $50 \%$ of inpatient admissions, and up to $50 \%$ of outpatient visits in places where malaria transmissions is intense” (qtd. in Hahn and Inhorn 35). The WHO adds that "Worldwide, Africa accounts for 9 out of every 10 child deaths due to malaria, for 9 out of every 10 child deaths due to HIV/AIDS, and for half of the world's child deaths due to diarrheal disease and pneumonia” (medicalnewstoday.com). The WHO also estimated that even though African nations spend close to US\$12 billion every year to combat malaria, malaria still kills one African child every 30 seconds (WHO Fact Sheet 94). Consequently, Africa accounts for 94 percent of the 850,000 children under five who die every year from malaria (GHC Fact Sheet). The WHO, however, attributes this high incidence of malaria and malaria-related deaths in Africa and other developing nations to severe socioeconomic factors and constant lack of sufficient economic 
resources. It made no mention, then, of the failure to adapt health-risk communication to the local culture- - which is the focus of this dissertation.

These gloomy statistics explain the endemic nature and effects of malaria on the socioeconomic development of many African nations, including Liberia. These statistics also show that global health risks are themselves in transition in that as the world's populations grow older, past successes against infectious diseases such as malaria and TB tend to dissipate (i.e., chloroquine use) or multiply in their effectiveness (GHR 6). For example, “30 years ago malaria had been eradicated or dramatically reduced in 37 countries...but this situation has been rapidly reversing, especially over the last decade” (MFI Fact Sheet, 1997). This changing trend explains why the WHO now sees global health risks as demanding the full support, participation, and cooperation of all peoples, nations, and cultures. It argues that health and wellness in the 21st century demand "a shared responsibility, involving equitable access to essential care and collective defense against transnational threats” (GHR). In the last ten years, several international cooperative efforts aimed at combating malaria and related diseases have been instituted in developing nations in sub-Sahara Africa, Asia, and other parts of the world. These efforts include PMI, the Global Fund, the World Bank Malaria Booster Program, and The Roll Back Malaria (RBM) Partnership. Today-as a result of concerted international efforts and funding commitments through such programs - the annual funding commitments for global malaria-treatment-and-control activities have risen from under US\$50 million prior 2000 to about \$1.5 billion in 2007 and to about $\$ 6$ to $\$ 7$ billion per annum beginning in 2009 for the next 10 years (Phillips 2010).

Moreover, in May 2009, the U.S. government launched The Global Health Initiative (GHI) to help reduce the burdens of malaria and other global health risks in communities across the world .The GHI is a six-year (2009-2014), \$63 billion funding commitment in support of global health activities, including \$51 billion for support of existing global HIV/AIDS, tuberculosis, and malaria programs and \$12 billion for maternal and child health and neglected tropical diseases programs worldwide (USAIDLantos-Hyde 5). Operating alongside GHI is PMI, originally a five-year (2005-2010), 
$\$ 1.2$ billion health initiative launched by the U.S. government to combat malaria in 15 countries in sub-Saharan Africa, including Liberia, that have high mortality rates from malaria. In 2008, the U.S. Congress passed the Tom Lantos and Henry J. Hyde Global Leadership against HIV/AIDS, Tuberculosis, and Malaria Act (or Lantos/Hyde Act), which increased PMI funding from the initial \$1.2 billion per year in 2005 to \$5 billion per year for fiscal years 2009-2013. PMI is one of the MTCPs in Liberia analyzed for this dissertation.

Notwithstanding these cooperative partnerships and huge financial commitments or spending earmarks, the key question that still evades both experts and laypeople is Why is a preventable and curable disease like malaria still topping the global health charts in lethality? There is certainly no easy answer to this question. As the United States Agency for International Development (USAID) notes in its malaria case management operations manual, "the relationship between knowledge and action is complex" (MCMOM 50) due to cultural and individual perceptions of risk. For even if two individuals or two cultures have identical knowledge about what a specific risk ought to be, how each individual or culture reacts to that risk is bound to differ greatly based on individual and societal perceptions of the severity of the specific risk. This is why in operationalizing risk communication — or any other form of oral, written, or visual communication aimed at behavioral change,- - the key strategies should include the targeting of community members, village leaders, youth leagues, women's groups, and civil and religious entities for active participation in the communication process.

\subsubsection{Malaria Treatment and Control in Liberia}

Due to its vast wetlands and tropical rain forests, Liberia is a hotbed for malaria. The country is home to the four, major, globally known malaria parasites, including the deadly Plasmodium falciparum. In 2005, the Liberia Malaria Indicator Survey (MIS) indicated that 66 percent of children under five in Liberia were infected with Plasmodium falciparum (MIS Update 2009, 1). The 2005 MIS also indicated that "only $3.2 \%$ of children under-5 with fever received first-line treatment for malaria within 24 hours (see Figure 1.2 for regional breakdown of percent of children infected with 


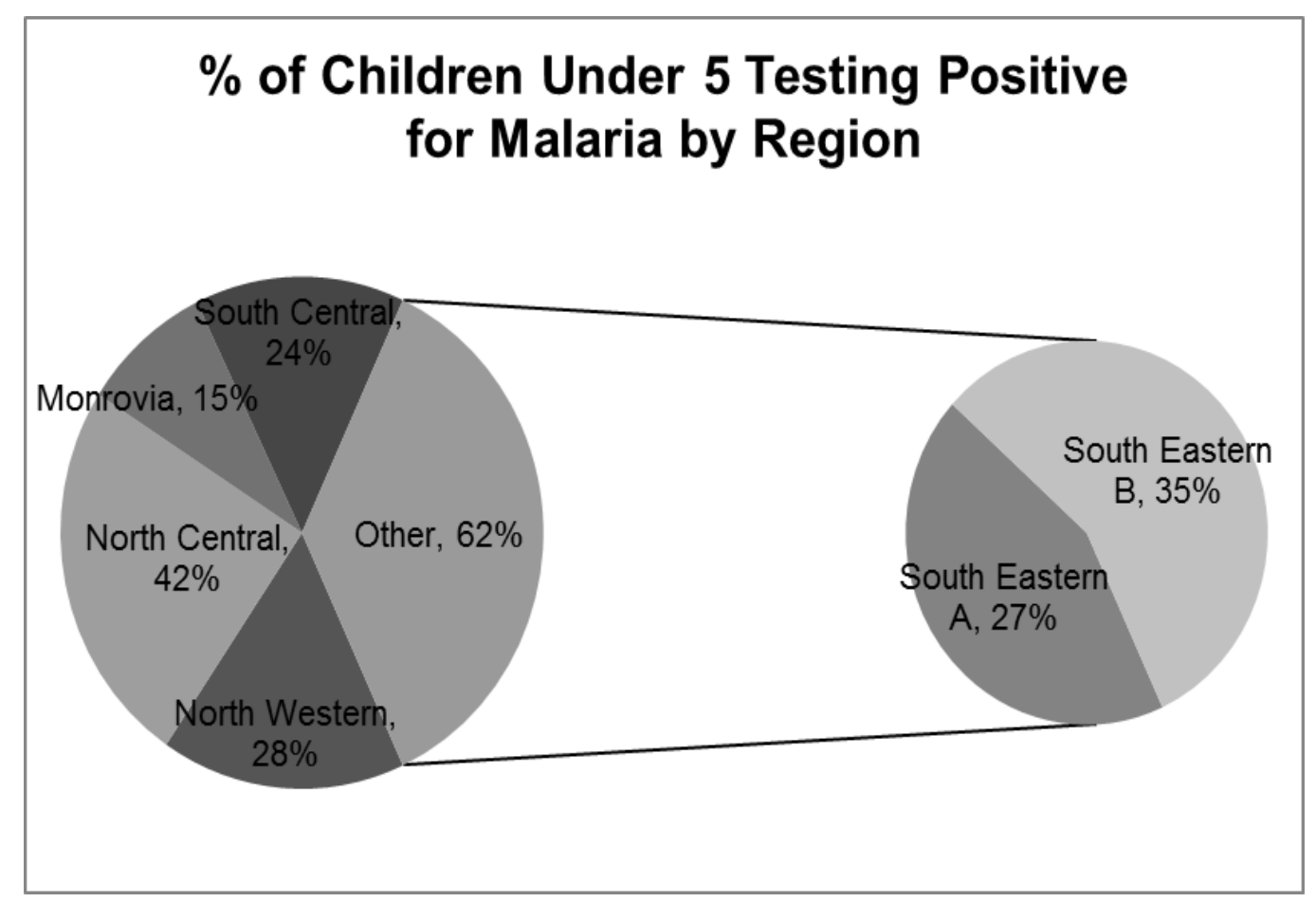

Figure 1.2: Distribution Map of Malaria in Children in Liberia

malaria), and only about 4\% of pregnant women received any kind of treatment during their pregnancy” (“2008 MOP” ). The MIS estimates that all of Liberia’s 3.8 million people are at risk of contracting malaria, including about " 565,000 children under five and 188,500 pregnant women” (“2008 MOP” ). Moreover, in 2008, Liberian Health Ministry officials disclosed that "in Liberia, malaria is the leading cause of morbidity and mortality, accounting for over 38 percent of all out patient consultations, 44.3 percent of inpatient deaths and is reported to cause approximately 21,000 deaths among children under five years of age” (The Analyst ).

These statistics alarming, and the situation is made all the more acute by Liberia's weak health infrastructure, shortage of health workers, lack of vehicles, and damaged infrastructure resulting from the country’s 1989-2003 civil war ("MOP” 2008). A 2007 report by the United Nations Development Assistance Framework in Liberia (UNDAF) presented the following description of healthcare delivery services in Liberia: 
The health care system and health workforce are fragmented, uneven and heavily dependent on programmes and international nongovernmental organizations (NGOs). Out of 389 health facilities considered "functional," almost 300 are supported by NGOs. With less than 10 per cent of Liberia's population having access to health care, revitalizing the health system is a major challenge facing the government. (UNDAF Report 23)

This bleak picture of current healthcare services in Liberia is largely accurate. At the end of the civil war in 2003, up to 95 percent of healthcare delivery services and infrastructures in Liberia were destroyed, disrupted, and/or degraded to the point that many hospitals and clinics were hardly functioning (BASICS). Dr. Joel Jones, head of Liberia's Malaria Control Program, confirmed this situation in his keynote address on the state of malaria control in Liberia at the 2005 Liberian History, Education, and Development, Inc. (LIHEDE) conference in Greensboro, North Carolina. Jones said Liberia was entangled in "a situation where when 50 patients entered a clinic, 25 of them are malaria infected," and that unless his agency collaborated with other agencies locally and internationally, "it will be a challenge to combat malaria in Liberia" because "The lack of adequate data collection has affected the record of adequate information for effective treatment programs” (LIHEDE Press Release). These shortages and forecasts also explain why by 2007, international NGOs and other aid agencies were providing more than 80 percent of healthcare services in Liberia (2008 MOP), and up to 2011 healthcare delivery services in Liberia were still under the virtual control of international NGOs and other aid agencies. All 15 counties or political subdivisions of Liberia still face a "year-long, stable, malaria transmission" problem (2008 MOP), which exposes the entire population to malarial infection.

This exposure to malarial infection has resulted in formal and informal approaches to malaria treatment and control in Liberia. During most of the 1960s, 70s, and 80s while I was still a child, teenager, or young adult in Liberia, the most common malaria treatment options consisted of Chloroquine tablets and injections, Fansidar tablets, and a concoction of various herbal substances, depending on geographical 
location. The well-known herbal substance for treating malaria and fever across Liberia at that time was jologbo, a bitter root, sold in the open markets. In those days, insect repellents such as mosquito chords and sprays were used in the homes before bedtime, and occasionally selected chemicals (e.g., DDT) were used as external treatments of mosquito-breeding sites, such as contaminated ponds and open-air dumpsites, in an effort to contain and prevent the spread of mosquito, the main malaria-carrying parasite. In post-civil war Liberia, some people still treat malaria with jologbo and other local and foreign herbal substances, such as Salsa leaves, pawpaw leaves, and ganagana. However, the emphasis has mostly shifted to a combination of "lifesaving drugs," mainly Artemisinin-based combination therapy (ACT), and such preventive measures as indoor residual spraying (IRS) with insecticides, insecticide-treated mosquito nets (ITNs) or bed nets, and intermittent preventive treatment for pregnant women (IPTp). The 2009 MIS Update notes this trend, in Liberia, especially among women: "When asked what drugs are used to treat malaria, half of women mentioned chloroquine and/or the 'new malaria drug,' Artesunate-amodiaquine combination (ACT). Onequarter of women mentioned quinine, while 20 percent mention aspirin, panadol, or paracetemol. Only a tiny proportion of women reported SP/Fansidar as a drug used to treat malaria" (43).

Under these treatment options, "since 2005, Liberia has received more than halfa-million ITNs or bed nets from donor agencies. Of this number, more $660,000^{1}$ bed nets were donated free of charge door-to-door, with target distribution of at least three ITNs per household or one ITN for each sleeping space” (2008 MOP). Private sellers are also involved with bed net distribution in Liberia, but the 2005 MIS found that although about 18 percent of Liberian households owned a bed net (not necessarily an ITN), only 2.6 percent of children under-five had slept under an ITN the previous night (2005 MIS 2). In contrast, the 2009 MIS indicates not only that a little over one-quarter of children (at least 26 percent) slept under an ITN the night prior to conduct of the

\footnotetext{
${ }^{1}$ The number of bed nets distributed since 2005 is higher than bed nets received, which might be that additional nets were received from other donors for the same period, or that the number is in error. The data is correctly quoted from the 2008 MOP in both cases of the bed nets received and comparison of ownership of ITNs and other types of bed nets.
} 
survey, but also that 49 percent of households in Liberia had at least one bed net (treated or untreated), and another 19 percent had more than one bed net (2009 MIS).

However, with 85 percent unemployment and a vulnerable population still recovering from the residual effects of civil war, Liberia is seriously lacking in healthcare delivery and related social services. As a result, the WHO, the Global Fund, the United States Agency for International Development (USAID), the United Nations (UN), and several other international NGOs and aid agencies are heavily involved with MTCPs and related healthcare services in Liberia. International NGOs and other aid agencies spend in excess of US\$40 million (an amount equaled to about 15 percent of Liberia’s 2009-2010 national budget of US\$371 million) each year on MTCP activities in Liberia in four core areas: IRS, ITNs, IPTp, and Lifesaving drugs or ACT. However, while it is important to recognize the invaluable role and humanitarian work of international NGOs and other aid agencies in facilitating healthcare-delivery-and-support services in post-conflict nations like Liberia, from a practical standpoint, it is not possible for these NGOs and aid agencies to deliver healthcare services in Liberia and other African countries forever. Therefore, the task of providing continued healthcare-delivery-and-support services will, in time, have to revert to the national governments and local populations. Hence, the strategies for communicating health risks to diverse populations in Liberia and elsewhere in Africa must depend largely on the active participation of the citizenry. This means that healthcare-delivery services that are not adapted to local culture and traditions will not achieve the desired results, regardless of the amount of money spent.

Despite the pressing need for cultural adaptation, evidence of the involvement of the local population and inclusion of local culture and traditions in the design and implementation of MTCPs is scanty or nonexistent. Although the National Malaria Control Program has recently begun to enlist the help of traditional leaders in the dissemination of malaria-control information in Liberia, the role of these traditional leaders has been limited to local language advocates/interpreters and instruments of behavior-change communication. This is the case because each of the four core areas of focus of MTCPs in Liberia is based on methods and procedures designed outside Liberia. The head of the National Traditional Council of Liberia, Chief Zanzar Karwor, bemoaned this trend as recently as January 2010, 
and warned that "no [MTCP] will succeed in [Liberia] without the input of traditional people” (Star Radio Liberia). In fact, the lack of active involvement of the local people in the design and implementation of MTCPs in Liberia has meant that many of these MTCPs have met with limited success. Furthermore, the MTCPs have lacked a strategic health-riskcommunication component that is essential to alerting the local population to existing or impending health risks in order to solicit their involvement in confronting and controlling such risks. That much is acknowledged in the 2009-2013 Malaria Strategic Plan for Liberia (20).

Hahn and Inhorn argue that "local populations, not the outsiders, are the experts on their own sociocultural environment" (9); hence, involvement of local populations in the design and implementation of global health services and programs is essential to program success. According to Hahn and Inhorn, in spite of the relative impact of globalization on public health in modern societies, local beneficiaries of global health programs have a tendency to "refashion or resist global forces" (15). Hence, to avoid unnecessary health risks, public outrage, and cultural resistance, it is important to ensure full participation of the local people in the design and implementation of healthcare programs. Incorporating the local people's cultural values, traditions, and mores into program design will minimize the cultural differences, taboos, and practices that have the potential to derail any health ventures.

Therefore, in examining MTCPs in Liberia, it was important not only to find out what effects local Liberian culture and traditions have on the process of communicating health risks to affected populations, but also to understand what underlying cultural and social factors promote or hinder the incorporation of local culture into strategies for designing and implementing MTCPs. As noted earlier, because different programs report their results differently, I evaluated program outcomes in light of possible inaccuracies in program reports and goals, confounding variables, and levels of cultural adaptation. In cases where there is resistance to MTCP antimalarial strategies (such as bed-net use) because traditional methods are believed to be adequate or superior, I have provided a record of these traditional, competing methods. 


\subsection{Risk Communication and the Cultural Impact of Risk: A Review of the Literature \\ Section Summary}

In this section, I review the current state of risk research (3.1), debates about risk and cultural representation (3.2), various health-risk paradigms and theories (3.3); the role of worldviews in risk communication (3.4), and the impact of health beliefs and culture in risk communication (3.5). This review provides a grounding in the issues, challenges, and prospects for designing and communicating health-risk information across diverse cultures and populations.

\subsubsection{Debates about Risk and Cultural Representation}

Lupton argues that "debates about risk always involve questions of cultural representation and meaning and political positions" (30). She explains that more often than not, "expert knowledges tend to contradict each other, resulting in debates over standpoints, calculation procedures, and results" (64). Lupton contends that questions of cultural representation and meaning become more profound in risk situations insofar as “what is deemed a 'danger' or 'hazard' in one historical or cultural context may not be so identified in another"(30). And she contends that this has wider implications for "how knowledges and understandings about risks are developed" in individual societies and cultures (30). Lupton acknowledges that "judgments about risk are not simply cultural interpretations of objective dangers and hazards" (30); instead, they are an exercise in magnitude, care, and caution that takes into account the idea that risks are not static but constantly changing phenomena (29). 
With respect to the questions of cultural representation and meaning of risk, Fischhoff et al. indicate that "Often the breadth of a hazard [or risk] category becomes apparent only after the decision has been made and its implications experienced in practice” (10). For the categorization of a hazard (i.e., a danger or any situation that could cause loss of life or injury) or risk (i.e., any probability of adverse outcomes) as potentially harmful or less harmful is based partly on the health and political interests of each society. In other words, it may not be prudent to dismiss outright as "respondents' ignorance” (Fischhoff et al. 28) the public's answer to a particular question intended to gauge the public’s perception or knowledge of a hazard. As Lupton notes, "the central tenet underlying cultural understanding of risk is that 'in all places at all times the universe is moralized and politicized" (46).

As Fischhoff et al. point out, "Unfortunately, the elicitation effects that bedevil the study of people's values may be just as potent in affecting their judgments of risk" (28). It is within the context of elicitation that specific theoretical perspectives, approaches, and paradigms become very important in discussing the cultural impact of risk on society. Four such approaches, theories, and paradigms dominate practices in health education and health-risk communication today: psychometric paradigm of Paul Slovic et al., the sociological perspective of Ulrich Beck, the psychosocial-epidemics paradigm of Philip Strong, and Cultural Theory (or grid/group analysis) of Mary Douglas et al.

\subsubsection{Health-Risk Paradigms and Theories}

The psychometric paradigm, which uses "psychophysical scaling and multivariate analysis techniques to produce quantitative representations of risk attitudes and perceptions" (Abraham 11), is the dominant paradigm on which "WHO's outbreak communication guidelines, as well as the national communication plans of many countries are based” (Abraham 1). This paradigm mainly tries to explain differences in risk perception between technical experts and the public by focusing both on how individuals in society perceive and react in risk situations and on how the judgments 
underlying such reactions are made in the face of the potential dangers of particular risks.

The sociological perspective looks at the aggregate of the social and political impacts of risk on society resulting from rapid modern technological advances. In this paradigm, Beck acknowledges the invaluable contributions of both a cognitive-science perspective (or scientific-objectivism) and a strong social-constructionist perceptive (or cultural relativism) to risk information gathering and distribution. However, Lupton argues that scientific-objectivism's quest for "neutral objectivity" may have failed "to recognize the ways in which 'scientific facts,' like other views on risk, are situated and interpreted in cultural and political contexts" (60). The sociological perspective also holds that factors of risk that concern "one social group in one historical era may not worry another” (Lupton 61) in a different historical era. For example, malaria, polio, and related diseases are still diagnosed and treated in contemporary Western and nonWestern societies, but they may no longer raise the same levels of fears, concerns, and confusions they once did due to advances in technology and the availability of treatment options, such as vaccines and drugs. The focus of Beck's work, however, is on the need for a new sociological perspective that accounts for the strengths and weaknesses of both the social-constructionist perceptive and the cognitive-science perspective in the process of risk analysis, management, and communication.

For Philip Strong, it is undisputable that various kinds of risks-technological, biological, and environmental risks_-pervade human society on a daily basis. However, what is important to Strong is the epidemic characteristics of risk. He examines the social and psychological impacts of risk on human society and consciousness to construct the psychosocial-epidemics paradigm, which breaks down infectious diseases into three distinct psychosocial, epidemic risk categories: the epidemic of fear, the epidemic of explanation, and the epidemic of action. The epidemic of fear considers issues of suspicions by and amongst friends, neighbors, relatives, professionals, and other members of society as to the origin of a particularly infectious disease. The epidemic of explanation looks at attempts by society to get a clear understanding of the causes, scope, and impact of a particular disease on society (Abraham 12). Finally, the 
epidemic of action looks at ways and means by which to control a particular disease and recommends steps by which the disease could be eradicated. This aspect of the epidemic of action primarily falls on public and private individuals, and secondarily on risk experts and other members of society.

A fourth paradigm, Cultural Theory or grid/group analysis, looks at the sociocultural aspects of risk in society. Cultural Theory holds in part that "distinctive conceptions of human follow from particular beliefs about the world," and that embedded within these beliefs are "distinctive preferences for different patterns of social relations” (Coughlin \& Lockhart 37). These social relations and beliefs are expressed in four distinct worldviews: fatalism, hierarchy, individualism, and egalitarianism. Each of these worldviews suggests unique ways of dealing with risk. Fatalists think whatever happens in life is preordained by some powerful, supernatural force, like the Christian God (Slavoc 694). Hierarchists think that society can maintain, create, and recreate itself through orderly, downward flow of commands from designated societal authorities (Slavoc 694). Individualists like to be left alone to act in ways that satisfy their best interests without any constraints, whether from government or other sources (Slovic 694). Egalitarians believe in free expression and think "natural and social environments are sufficiently robust to support some exploitation, but if humans press too hard, disaster is apt to follow" (Coughlin \& Lockhart 51). Egalitarians also tend to prefer a world in which power and wealth are more evenly distributed (Slovic 694).

The grid/group paradigm regards risk perception as beliefs situated in each of these worldviews. Through this appeal to the unique worldviews and cultural values associated with each group, "grid-group theory provides a structure for helping us understand varying patterns of attitudes in a way not possible within the confines of the unidimensional measure of political ideology” (Coughlin and Lockhart 51). Hence, political ideology plays a pivotal role in the articulation of cultural values in society, including cultural biases, which "are based on beliefs about the natural and social environments that rest ultimately on experience” (Coughlin and Lockhart 37). 
Given the roles of political ideology and individual experience in risk knowledge and analysis, Douglas believes that lay risk knowledge and corresponding reactions to risk should be acknowledged for their "use and value within a particular cultural context" (Lupton 37), as opposed to being seen as "erroneous or biased as if they differ from expert assessment” (Lupton 37). To Douglas and other proponents of the grid-group thesis both expert knowledge and lay knowledge are important to identifying, treating, preventing, and eradicating health risks, in spite of the different philosophical and cultural outlooks and approaches. Lupton seems to suggest that risks should be regarded "as shared conventions, expectations and cultural categories...founded on clear cultural functions and responsibilities" (38), in that every society uses particular cultural frames to decide which risks should be acted upon with "anxiety, fear, and anger" (39) and which should be neglected or ignored.

\subsubsection{Worldviews as Defining Factors in Risk Communication}

Because worldviews influence individual risk perceptions, how each individual manages risk has an impact on that individual's quality of life (Sellnow et al. 3). Hence, Sellnow et al. suggest, that in order for risk communication to reach its potential it must consist of an interactive process that gives all parties access to multiple messages representing all relevant worldviews (17). Differences in worldviews and cultures, however, "can and do present major barriers to effective health care intervention” (Huff and Kline 6).These differences may also result in adverse impacts on health-risk communication, especially in instances where health practitioners might "overlook, misinterpret, stereotype, or otherwise mishandle their encounters with those who might be viewed as different from them in their assessment, intervention, and evaluation planning processes” (Huff and Kline 6). These complexities in risk perceptions and interpretations best explain why scholars like Wilkelman believe that the concepts of cultural awareness, cultural difference, cultural sensitivity, and cultural competence (20) are germane to minimizing the challenges posed to health-risk communication by differences in risk perceptions, cultures, and worldviews. 
According to Wilkelman, cultural awareness is an individual's acknowledgement of the existence of a culture other than his or her own; culture difference is how well each person is able to cope with another person's culture; cultural sensitivity is the ability of peoples of two cultures to recognize and respect the symbolic differences in their body language and manner of speaking; cultural competence is how a person from one culture is able to function effectively within another culture; and cultural proficiency is the ability to transfer cultural knowledge and skills to others outside of one's culture (20). In essence, cultural awareness, sensitivity, competence, and proficiency speak to the varying degrees and levels at which peoples from different cultures are able to interact with one another with clear understanding, respect, and appreciation of one another's cultural values and mores. The ability to acknowledge and respect other people's cultures as well as to learn to interact with people of different cultures in spite of obvious cultural differences is also vital to the success of communicating health risks to diverse populations and cultures.

The impact of the diversities in language, culture, ethnic, and religious outlooks on health and wellness across societies is emphasized by communication professor Mohan Dutta, who argues that "How and what we communicate about health is embedded in our taken-for-granted assumptions about what it means to be healthy, what it means to be ill, and how we approach disease and illness" (2). Hence, each society or culture has some intrinsic values that shape its socioeconomic, political, health, educational, and moral direction and wellbeing. These factors are emphasized in the strong thesis of the social-constructionist perspective, which, like cultural relativism, holds that "cultures (the systems of beliefs, values, and norms of behavior found in all societies) are more or less coherent, systematic, and rational within their own contexts" (Hahn and Inhorn 8). Culture provides the framework for a much broader understanding of the social forces of society and their interactions, and it orients an individual member of society toward accepting risk messages only if that individual's "cultural construction of the disease [or hazard underlying the risk message] is similar to that of the communicator" (Abraham 3). Hence, respect for individual and societal perceptions 
of risk is essential to the success of any risk communication scheme, especially when evaluated in terms of the cultural impact of risk.

According to the authors of a 2000 Canadian Food Inspection Agency (CFIA) report on risk communication and government, "the relationship between the source of the communication and the recipient must be acknowledged as an important factor in effective risk communication, if not the most important factor”(Chartier and Gabler). And according to Dutta, "as a material entity, culture ...offers a defining framework for the articulation of cultural identities, beliefs, values, and practices” (80). Therefore, given the complex nature of culture-especially in terms of articulation (i.e., "the way that different things [values, feelings, beliefs, practices, structures, organizations, ideologies, and so on) come into connection or relation at a particular historical conjuncture”] (Slack 225)—risk communication has been beset by problems with culture and human perceptions of risk since it emerged as an academic discipline in public policy in the health and environmental sciences in the mid-1980s .

In their attempts to explain the relationship between risk communication and culture, and the intervening cultural and linguistic factors that influence risk and risk perception in individual societies, Lundgren and McMakin note that "Laws about what constitutes a risk, and how to communicate them, vary from country to country” (333). Country-specific risk laws and related cultural practices and preferences highlight the complexities, challenges, and prospects for communicating health-risk messages across diverse cultures and populations. Every human is born into a particular culture, and it is through this culture that he or she cultivates a worldview, explores the environment, and identifies health risks. Our culture affects the way in which we communicate with people of other cultures and how we make choices about "what we eat, how we protect and expose ourselves, [our] patterns of sex and procreation, our hygienic practices, how we bond together, and [our] life-style behaviors” (124). In essence, culture provides the “core conceptual framework” (Winkelman 20) for understanding all human health and related risk behaviors, and it is the primary driving force behind how risk is perceived, communicated, and treated in individual societies. Indeed, how we look at and think of the world is influenced greatly by the cultural patterns that construct our individual 
beliefs, values, and norms. For culture, according to Winkelman, is "vested with an inherent power to demarcate risk perception among individuals in society in spite of what each culture considers harmful or unharmful” (7). The attempt to understand this inherent power of culture over matters of risk and risk perception has given rise to such theoretical approaches as Dutta's culture-centered approach and Hochbaum, Rosenstock and Kegels' Health Belief Model.

In articulating his culture-centered approach to risk communication, Dutta says, "communicating about health involves the negotiation of shared meanings embedded in socially constructed identities, relationships, social norms, and structures” (55). These identities and shared meanings are constructed mainly through "active participation of community members" (Dutta 55) who more often than not draw on their shared experiences about health and wellness in society for that purpose. This approach assumes communication about health to be "culturally situated and ... simultaneously tied with structural processes” (Dutta 76). Therefore, in order to understand the health experiences of members of each cultural community, one must first endeavor to understand the relationships and "interactions between structure and culture" within each society (Dutta 76). This is why Kim and Gudykunst suggest that "interpretive approaches to culture and communication need not, and probably should not, adopt a relativist ethical stance in assessing the quality of communication across cultures" (41). For while culture is dynamic rather than static, more often than not, each culture is dynamic in terms of effecting change only insofar as permitted by local socioeconomic conditions and politico-cultural contexts.

In these respects, the culture-centered approach seeks to engender understanding of "the ways in which the health experiences of the cultural members are marginalized, constructed, as deviant or abnormal" in the face of such constraints as limited access to appropriate healthcare resources (Dutta 85). Underlying the culture-centered approach is the notion that issues of health risks, risk perception, and risk behaviors are dependent more on practices within individual cultures than on the preferences of individual persons. 
Kim and Gudykunst claim that "every communication event is to some degree intercultural” (37), insofar as at any given time, a communicator's behavior is reflective of his or her "experiential background," which ultimately "shapes the attributes of his or her internal meaning” of things and perception of the world (49-50). The communicator's background is shaped greatly by culture in the sense that culture defines "the logic of communication itself" and provides the "rules, schemas, scripts, and values used in communication” (Kim and Gudykunst 49-50).

By this token, culture becomes indispensable to any communication process, especially in the case of "direct, face-to-face communication encounters between and among individuals with differing cultural backgrounds” (Kim and Gudykunst 13). In other words, communicating health-risk messages to peoples of diverse cultures and populations requires some understanding of the cultural contexts and complexities of the interaction. Such an understanding is necessary because "Those who interact with foreign cultures have a moral obligation to take those cultures seriously, including their social organization and values” (Hahn and Inhorn 10). Every society has a set of sociocultural value and organizational structure that governs its wellbeing. Accordingly, it behooves everyone who interacts with people of a foreign culture to learn to recognize and respect those different cultural perspectives and values whenever they are engaged in intercultural exchanges and interactions that involve the gathering of risk information and the promotion of risk avoidance.

\subsubsection{Effects of Health Beliefs and Culture in Risk Communication}

Today, the WHO and other international health organizations and funding agencies usually recommend the use of bed nets and ACT as treatment options for malaria control. But elements of culture are always present in any health-risk communication situation, whether explicitly or implicitly. Hence, the cultural backgrounds, beliefs, and perspectives of decision-makers in these international organizations and funding agencies are more likely to tip the balance in favor of what health-promotion and risk-communication strategies to pursue. For example, almost any health-promotion and risk-communication programs designed in the United States and 
other Western nations are bound to pursue a strategy geared toward behavior change based on the influences of health promotion theories such as the Health Belief Model (HBM), which was first developed in the United States in the 1950s to confront cultural and related practices affecting health behaviors in the United States.

As part of the psychometric-risk-analysis paradigm under the cognitive-science perspective, Lupton describes HBM as dominating "ideas about risk perception in the fields of health promotion and health education" (21). She contends that HBM relies on individual action in the recognition and reduction of risk in the sense that in order to act in particular risk situations, "individuals must find themselves vulnerable to the [risk or] threat, they must perceive the threat as having serious consequences, they must believe that taking preventive action will be effective and they must believe that the benefits of the action will outweigh the cost” (Lupton 21). Thus, understanding the role of individual beliefs, choices, and preferences in the creation and dissemination of risk information and health-risk messages is essential to adapting health-risk communication to the special cultural contexts of diverse populations.

While the HBM provided "an overarching framework for promoting preventive behaviors” (Witte, Meyer, and Martell 36), it still reflected the cultural perspectives of the theorists who conceived of it based on their experiences with health practices in the United States at the time. HBM is regarded as "the grandmother of most modern health education theories” (Witte, Meyer, and Martell 36), and its principles have been wholly embedded in many risk-message models in the contemporary world. The incorporation of HBM principles in crafting risk messages makes HBM essential to the cultural impact on risk communication because HBM attempts to explain and predict healthy behaviors by focusing on individual and societal attitudes and beliefs regarding individual health choices and related risks. HBM originally focused on four primary constructs—perceived susceptibility, perceived severity, perceived benefits, and perceived barriers - which together sought to engender understanding of perceived threat and its associated net benefits to both an individual and society. These four constructs dominated discussions about health-promotion practices and individual or societal reactions and feelings toward health-risk issues until the introduction of two 
new constructs in the 1990s: cues to action and self-efficacy. Cues to action looks at an individual's readiness and ability to act in any health-risk situation, while self-efficacy looks at the level of confidence that an individual is likely to act in a health-risk situation and why and how he or she takes one action rather than another in each situation (utwent.nl).

\subsection{Theoretical Models of Risk Communication}

According to Covello et al., "Risk communication is based on four theoretical models that describe how risk information is processed, how risk perceptions are formed, and how risk decisions are made”(6): risk perception, mental noise, negative dominance, and trust determination. The Risk Perception Model looks at individual, organizational, or societal perceptions of risk based on 15 common risk factors: voluntariness, controllability, familiarity, catastrophe potential, dread, ethical/moral nature, benefits, understanding, equity, reversibility, trust in institutions, personal stake, human versus natural origin, and uncertainty (Covello et al. 16). Each of these factors not only contributes to but also helps determine issues of fear, worry, anxiety, hostility, or outrage at the individual, community, or societal levels, in terms of the degree to which risk is perceived, evaluated, and mitigated. Together these risk factors are called “outrage factors” (Slovic and Weber 17), which are fundamental to Peter Sandman's formulaic expression, "risk = hazard + outrage" (Sandman).

The Mental Noise Model looks at "how people process information under stress and how changes in how information is processed affect their actions" (Slovic 17). The Negative Dominance Model looks at how "negative and positive information in highconcern situations” is processed (Slovic 17). The Trust Determination Model looks at the level of trust that is projected and maintained throughout any risk-communication situation, since trust is not only a "common thread in all risk communication strategies" (Slovic et al. 17), but also a key ingredient for any person-to-person communication. 
While these four theoretical models speak directly to how risk perceptions and decisions are formed and how risk information is processed, much risk communication, especially in the health and environmental fields, is also rooted in the rational model of risk. According to Alaszewski, "Communication grounded in the rational actor model emphasizes the role and position of experts such as doctors who have the ability to identify relevant risk knowledge” (103). One of the most basic assumptions of this model is that risk communication can be treated as a linear process in which "the flow of knowledge from the knowledgeable doctor to the uninformed patient” (Alaszewski 11) or from the knowledgeable expert to the uninformed layperson is sufficient to mitigate a risk arising from natural disasters and related health outbreaks. Hence, under this model, "When patients appear to be making irrational or harmful decisions, for example, continuing to smoke, choosing not to vaccinate a child against measles, mumps and rubella, or not complying with medication, the professional's response is to work harder to convey the risks” (103). This model assumes that expert knowledge of risk is more accurate than and preferable to corresponding lay knowledge. This is the sort of social interaction or exchange of information that Waddell describes as the OneWay Jeffersonian model, which assumes that "the public has a right to participate in decisions that affect its wellbeing, but that it should be empowered to do so, simply and unproblematically, through a one-way transfer of expert knowledge” (“Defining” Waddell 207). Both the rational model and One-Way Jeffersonian model seem to postulate that the public and other laypeople are "obtuse, uninformed, ignorant, and histerial about issues of risk” in terms of risk knowledge (Sellnow et al. 24), even though the public's reaction to risk may be a consequence of legitimate concerns arising from individual past experiences and related cultural, religious, or philosophical beliefs and practices. 


\subsubsection{Rational Risk Model and Human Social Behaviors}

The rational model and One-Way Jeffersonian model contain assumptions about human social and cognitive behaviors that fail to acknowledge that laypeople and other members of society have legitimate concerns that should not be subordinated to expert knowledge. Members of society always want to "feel safe and protected” (atsdr 5) about the particular risk message or health information they receive. As Hahn and Inhorn have observed, risk information may be rejected by members of a society either because they do not understand the information or because they do not prioritize the information and its potential benefits (10). In this context, Alaszewski believes the rational model is flawed in many respects, in part because "Risk knowledge cannot actually be used directly by patients to inform their decision making” (11). He says that while many experts treat risk knowledge as "a single uncontested source of knowledge that is relatively easy to access... in reality risk knowledge is often a complex matter” (16).

This reality is manifested through expert disagreements over risk findings and potential risks impacts, which are commonplace in risk communication practices. Scientific risk assessment is more an expression of probability than an act of certainty, since the goal of such a research is to generate epidemiological data about the risky behaviors of people or the probable harmful effects of particular risks on particular populations without necessarily accounting for uncertainty, the unknown variable that usually tips the balance in how people perceive risks or choose to react to risk in particular risk situations.

\subsubsection{Uncertainty, Trust, and Credibility in Risk Communication}

Embedded within the concept of uncertainty, of course, is the dual issue of trust and credibility. Trust often deals with whether or not the source of a piece of information is reliable, but according to Bennett et al., in risk communication, trust also deals with issues of "perceived competence, objectivity, fairness, consistency, and goodwill” (17). Credibility, on the other hand, deals with the relevant subject knowledge and authority of the source of a piece of information. Hence, trust and 
credibility are essential components of all person-to-person communication, but Alaszewski says, "trust is central to risk communication” because humans are not passive but rather active beings who "actively seek information on risks from many different sources [i.e., friends, relatives, medical advisors, or the Internet], especially when they are aware that they are facing a crucial decision” (16).

Trust and credibility are somehow intertwined in terms of person-to-person communication because how people choose to react to a piece of information (healthrisk or otherwise) depends on how trustworthy and credible they believe the source of that information to be. Otherwise, inaction is certain, even with an abundance of information. Hence, since basic human communication demands a two-way interaction, and the exchange of health and risk information involves not only doctors and patients or experts and laypeople but also a diversity of people with complex backgrounds, both the rational model of risk and the One-Way Jeffersonian model seem to take for granted the issues of individual risk perception and the elements of individual trust and credibility. For many people-whether based on educational, racial, socioeconomic, or cultural factors and linkages or affiliations - the messenger rather than the message is the most important thing. Research indicates that individuals from some cultures prefer that risk and related information come from "trusted cultural agents rather than from technical experts and other providers of expert knowledge” (Sellnow et al. 23). Hence, as people seek risk information, they also tend to "make conscious decisions to avoid certain forms of information” (Alaszewski 16). As a result, Alaszewski says, "there is little evidence that risk communication based on the rational actor model shapes an understanding or behaviour in ways in which health-policy makers and public health experts want” (17).

Renn et al. acknowledge the varieties of "risk estimates among individuals and social groups” (9) along with corresponding but competing risk priorities influenced by individual sociocultural values and societal interests. Nevertheless, they argue that "The gap between experts and the public turned out to be transformed into numerous gaps among experts and among publics” (9). As a result, they claim that risk managers and other technical experts are beginning "to abandon the idea of public input altogether and 
to return to the safe haven of technical expertise” (9). Renn et al. do not predict how the question of expert knowledge of risk versus lay knowledge of risk will be settled, but they believe "a new era of expert domination in risk policies” (9) is well underway. Many social researchers and scientists have also begun to advocate that "social research on risk should be based on theoretical frameworks that risk managers and natural scientists would also find attractive” (Renn et al. 9).

Despite the current disputes about expert versus lay perceptions of risk, Slovic and Weber still do not see any significant "differences in opinions about acceptable levels of risk” between experts and laypeople (11). They believe the misunderstandings about expert knowledge and lay knowledge lie mostly in differences in "definitions of the concept of risk and...assessments of the magnitude of the riskiness of a given action or technology” between experts and laypeople (11). Drawing on the psychometric paradigm of health-risk communication, Slovic and Weber say many psychometric studies have shown not only that "perceived risk is quantifiable and predictable," but also that the concept of risk can mean "different things to different people” (11), including experts (e.g., expert disagreement) and laypeople (e.g., personal identity and experience). For while experts tend to rely on technical data (e.g., annual fatalities) to make judgments about risk, laypeople tend to make judgments about risk based on any of the 15 common risk factors discussed earlier. Hence, the whole notion of expert knowledge versus lay knowledge of risk becomes cumbersome upon the realization that the concept of risk is a human invention intended to help humans understand and cope with the dangers and uncertainties of life (Slovic 690).

\subsubsection{Universal Characteristics of Risk}

To Slovic, there is no such thing as "real risk" or “objective risk" (690) because risk is a bonafide part of human societies and cultures rather than an independent entity residing somewhere in the wilderness outside human involvement and control. Slovic says that because people differ in their social, cultural, and political attitudes and judgments about what constitutes risk and what does not, one would be lucky to find any universal set of characteristics for describing risk (692). This unpredictability about 
the universal characteristics of risk is best explained by earlier studies in risk perception that found that while experts and laypeople might agree about the fatalities that a certain technology produces on average in a given year, they are more likely to disagree about the degree of risk emanating from such a technology (Fischhoff 139). Fischhoff argues that while lay knowledge may not be organized as systematically as expert knowledge, the lack of systematic organization does not preclude people from having "some insight into where they go, how deeply they breathe, what they eat and drink, how long they shower, when they wash their hands, and so on” (143). He says that regardless of the source of a risk, exposure to any kind of risk is the result of human activities (143), so care should be taken to reduce some of the biases against laypeople as it pertains to risk perceptions.

Fischhoff suggests that in evaluating the strengths and weaknesses of lay beliefs and perceptions about risk, some key questions should be: 1) What does it mean when they [laypeople] perceive health effects that science has not established? 2) How should one treat their skepticism about an elaborate official evacuation or inspection or training plan [based on expert knowledge]? 3) Might they know something about what motivates people like themselves? 4) Do they have an independent perspective on what motivates technical experts, possibly clouding their professional judgment?” (143).

These questions seek to demystify the ongoing debates about expert knowledge versus lay knowledge in risk communication, so it is understandable why Fischhoff believes that communication should "be crafted to fill gaps, reinforce correct beliefs, and correct misconceptions - with some assurance that the messages are to the point and can be comprehended by recipients" (140). He also says communication should not tell people only those things that they need to know, but also take into account the specific details and circumstances of the recipients (140). He believes that understanding how certain kinds of risks are defined and treated in each culture and understanding how to structure a piece of risk communication to address the specifics of risk in each culture are essential to communicating health risks effectively. 


\section{Chapter 2: Linguistic Culture and Risk Communication}

\subsection{Introduction and Definition}

In this chapter, I look at linguistic culture and its effects on the delivery of health-risk messages to diverse cultural populations. Harold Schiffman first used the phrase linguistic culture in the late 1970s while studying the assimilation of GermanAmericans through instruction in English. Schiffman defines linguistic culture as "the set of behaviors, assumptions, cultural forms, prejudices, folk belief systems, attitudes, stereotypes, ways of thinking about language, and religio-historical circumstances associated with a particular language” (5). Among other things, linguistic cultures dictates how people within each society perceive and react to risks.

As a discipline devoted to various forms of health, safety, and environmental risks, risk communication addresses a wide range of global issues. Individual healthrisk messages, however, are locally produced and culturally situated, and they often reflect "the set of behaviors, assumptions, cultural forms, prejudices," etc. of specific communities. The various elements of linguistic culture listed by Schiffman interact with one another and help to facilitate and sustain social communication, including risk communication. Hence, such interactions are also explored in this chapter.

\subsection{Folk Beliefs as a Consideration in Risk Communication}

One of the key elements in Schiffman's definition of linguistic culture is folk belief systems, and it is essential to consider the role of such systems in the construction of health-risk messages. Folk beliefs play a central role in how health-risk information is crafted and disseminated among people in any society. Folk beliefs are also an integral part of linguistic culture because they are the superstitious hub of every society, and they provide the necessary local contexts for explaining the causes of illhealth and associated risks in society. For example, as part of the folk-belief system in contemporary Western cultures, "every death, every accident and every misfortune is chargeable to someone’s account” (Lupton 45); hence, someone is always made the 
scapegoat, found at fault, or held accountable under new and existing insurantial risk laws. Also, in 16th to 17th-century England, witches were seen as a source of misfortune in society and were blamed for causing all forms of illness and misfortune. Consequently, they were publicly burnt to death in attempts to abate such misfortunes (Helman 93).

Many studies have sought to establish a causal relationship between ill-health and folk beliefs. One study found that some people in the southern United States associated low blood pressure with "eating too many acid or astringent foods, such as lemons, vinegar, pickles, olives and sauerkraut” (Helman 92). Another study among low-income African-Americans found a tendency to attribute ill-health to the evil-eye or a sorcerer (Helman 93). Helman also claims that in developing nations, folk beliefs often associate poor health with low income per se, rather than with the conditions that generally accompany poverty, such as "the sort of food, water, clothing, sanitation, housing and medical care the people are able to afford” (4). Hence, folk beliefs about ill-health and other misfortunes are firmly grounded in the linguistic culture of each society, and they often exert tremendous influence on how the people of those societies identify and react to risks.

Folk beliefs about ill-health in Liberia are similar to those that Schiffman describes. For example, the 2007 Liberia Demography and Health Survey (LDHS) found a local folk belief that HIV was contracted through "mosquito or other insect bites... or by witchcraft or other supernatural means” (LDHS 2). Folk beliefs about how malaria and related diseases are transmitted are commonplace in Liberia, including beliefs that excessive exposure to the sun and excessive consumption of oranges can cause malaria. In spite of their lack of scientific validity, folk beliefs are shared and cherished traditions in many societies.

According to cultural theorist Raymond Williams, "Every human society has its own shape, its own purposes, [and] its own meanings” (4), which are transmitted or expressed through the language, arts, and institutions of each society. Reminiscing about his childhood experiences in a farming valley alongside the mountains, Williams writes: "To grow up in that country [valley] was to see the shape of a culture, and its 
modes of change," and "to grow up in that family was to see the shaping of minds: the learning of news skills, the shifting of relationships, the emergence of different language and ideas” (6). Williams’ notion of culture having special shapes, purposes, and meanings is consonant with Schiffman's idea of linguistic culture, which can also be described as the sum of the communicative and cultural practices of a given human society. Williams’ characterization of culture also suggests that understanding folk beliefs and related elements of a society's linguistic culture is key to effective communication within that society.

\subsection{Importance of Language to Participants in Risk Communication}

As human beings, we can use our senses of sight, smell, taste, touch, and hearing to create and acquire knowledge through observation and interpretation, but we cannot easily communicate with one another and exchange ideas and risk information without the benefit of language (spoken, written, or sign). Edward Sapir suggests that we usually "see and hear and otherwise experience very largely as we do because the language habits of our community predispose certain choices of interpretation” (69). Unraveling these language habits and choices of interpretation has become the preoccupation of critical and interpretative communication theorists and scholars. While interpretative theorists may feel contended with the ability to interpret a particular historic event or human activity, critical theorists are usually "not satisfied to develop interpretations of events; instead they imagine ways in which change and improvement can be attained" in order to extrapolate how "certain values are brought to bear on interpretation” (10). Interpretative and critical theorists believe that "language creates a world of meaning within which the person lives and through which all experience is interpreted” (Littlejohn 13).

Unlike interpretative and critical theorists, structural and functional theorists are more concerned with the "unintended consequences rather than purposeful outcomes" of human action and interpretations (Littlejohn 11). They believe that "the language and symbol systems used in communication have a life of their own apart from the people 
who employ these tools” (Littlejohn 11). In spite of their philosophical differences about what the role of language is and ought to be in society, all four categories of theorists generally see language as central both to human communication and to the creation of meanings.

The language we use "to communicate complex scientific issues" and risk information to one another, or to diverse audiences, is essential to our understanding of that specific issue or risk information (Bennett et al. 34). We are also able to "dissect nature [including health and environmental risks] along lines laid down by our native languages” (Whorf 1940, 213-4). Hence, whether or not we believe as individuals or as groups that risk is perceived and acted upon based on individual worldviews (see chapter 1), one of the key theses of the Cultural Theory of Mary Douglas, Aaron Waldavsky, et al. is the categorization of humans into four distinct worldviews: egalitarians, individualists, hierarchists, and fatalists. Each of these groups has demonstrable characteristics that impact how each group member identifies and reacts to risks and risk situations. Douglas claims that people are more likely to react to risk in particular ways because "each type of society has its custom-built ethical system" (qtd. in Lupton 38), through which certain dangers are selected for special attention by a society and entitled "risks" (Lupton 39).

This sort of selectivity makes sense to each cultural group based on the group's shared values and concerns. For instance, in order for one to communicate risk information to the fatalists, one must first endeavor to understand the values that fatalists hold dear and then tailor risk messages to those values. Consequently, fatalists would be more likely to respond positively to these risk-communication messages.

Usually, people's experiences with a particular form of illness are colored by much wider cultural and social forces within their society, such as folk beliefs, societal prejudices and stereotypes, and information offered by print and electronic media (Helman 88). Language is central to many of these communication channels: It is through language that we are able to exchange greetings with one another; share our innermost thoughts and feelings of love, hate, joy, and happiness with one another; and make laws that govern society at large. Hence-given its unique role in every human 
culture-language has both a special meaning and a life of its own. As philosopher Martin Heidegger says, "while language is not the only form of world view developed by human subjectivity, it is that form to which we must ascribe a special authority in the history of man's development by virtue of its formative power at each given time” (119). Heidegger argues that "In order to be who we are, we human beings [must] remain committed to and within the being of language, and [we] can never step out of it and look at it from somewhere else” (134). In other words, language is a situated byproduct of a specific culture, and the worldview of people within that culture is formulated to a certain extent by and through their language and the local geographic region and linguistic setting in which they are situated. Language enables humans to act in certain ways because "we do not merely speak the language-we speak by way of it" (Heidegger 124).

If we don't merely speak a language but speak by way of language, then the language we speak will ultimately determine how we communicate risk information. Yet the ability to speak a language may not be enough to facilitate understanding because "It is impossible for us to understand and interpret things without the mediation... of tradition, shared values, personal predispositions, and creative imagination" (Canagarajah 18). Tradition is the fulcrum on which language pivots, and speaking a language without understanding its shared traditions leaves much room for misinterpretations. Hence, in its 2009 Malaria Case Management Operation Manual (MCMOM), the WHO mandates that all national malaria-control programs ensure that all behavioral-change information and health-risk messages "be adapted to the situation and needs of the target groups and should be prepared in all the appropriate languages for the [targeted] country” (MCMOM 52).

The WHO mandate explicitly acknowledges the role of language and culture in the creation and dissemination of health-risk and related messages. However, in many countries, the use of local language and culture in the creation of health-risk messages has yet to receive much attention. For example, in their final report, the authors of the 2007 Liberia Demography and Health Survey (LDHS) rationalized their failure to translate survey questionnaires into local languages: "Given that there are dozens of 
local languages in Liberia, most of which have no accepted written script and are not taught in the schools, and given that English is widely spoken, it was decided not to attempt to translate the questionnaires into vernaculars" (LDHS 2). The claim that most local languages have "no accepted written script" is highly debatable: Vai and other local languages discussed in the next section ("Linguistic-cultural practices in Liberia") do have phonetic scripts for writing and related scholarly purposes. The absence of one or more local languages for disseminating public information and communicating health-risk messages in Liberia may have public policy implications far beyond the scope of this dissertation.

The effective use of shared language, folk beliefs, and worldviews in risk communication cannot be overemphasized. Since time immemorial, individuals, groups, and communities within human society have had to wrestle with various forms of risks for their own survival and well-being. Risk is sometimes regarded as "a socially constructed interpretation and response to a real danger that objectively exists" in each society (Lupton 39); however, the extent to which "real danger" or "real, objective risk" exists is highly debatable. Slovic (see chapter1, Section 1.4.3), rules out any possibility of the existence of "real risk" or "objective risk" in society, in that it is each society that identifies and defines what constitutes a risk and its severity or lack thereof.

On the other hand, Fischhoff, Watson, and Hope say that many technical experts in the field of risk communication recognize the existence of "objective" and "subjective" risks. They describe "objective risk" as "the product of scientific research, primarily public health statistics, experimental studies, epidemiological surveys, and probabilistic risk analyses," and "subjective risk" as "non-expert perceptions of that research, embellished by whatever other considerations seize the public mind” (31). Derby and Keeney argue not about objective versus subjective risk, or real versus artificial risk, but about acceptable risk. To them, what is most important is not whether risk is objective or subjective, but the extent to which society accepts and copes with particular risks and risk factors. They explain that "acceptable risk is not necessarily the level of risk with which we are happy” as a people, community, or society, but that which we are able to live with and manage (44). 
These debates are insightful notably in what they say but also in what they do not say. And what they don't say is that risk is a socially constructed phenomenon couched in individual cultures and worldviews. For instance, lifestyle risks (e.g., smoking and skydiving), natural risks (e.g., floods and famines) and technological risks (e.g., air and water pollution and chemical toxins) are risks that confront humans all of the time, but how each society reacts to these sets of risks depends largely on factors such as familiarity versus unfamiliarity and the consequent public "outrage" generated by such risks (Sandman). In other words, individual perceptions and worldviews are both part of a culture and "complicit in the production of risks and not simply responses to given risks" (Lupton 51). Such worldviews generally "shape what phenomena are singled out as 'risks' and how serious they are perceived as being” (Lupton 45). Hence, risk communication has become — and must be —an enterprising discipline that, in practice, interfaces daily with people of diverse cultural and linguistic backgrounds. The ability to communicate in a shared language and culture often removes from the communication process common communicative barriers, such as those associated with language interpretation, including misinterpretation, misunderstanding of contextual meanings, and outright misrepresentation. Without this ability, interactions involving people of divergent cultural orientations who employ different rules for governing the communication process could lead to serious miscommunication (Huff and Kline 14).

\subsection{Linguistic Cultural Practices in Liberia}

As indicated in the previous section, it is virtually impossible to adapt healthrisk communication to the specific cultural context of a particular society, such as Liberia, without first understanding the linguistic culture of that society. One of the key attributes that Schiffman includes in his definition of linguistic culture is "ways of thinking about language.” Among philosophers, anthropologists, social psychologists, linguists, and cultural theorists, many different theoretical perspectives and approaches underpin "the study of how language is used, and how members of a culture acquire and display knowledge of usage” (Schiffman 7). For instance, Ferdinand de Saussure, 


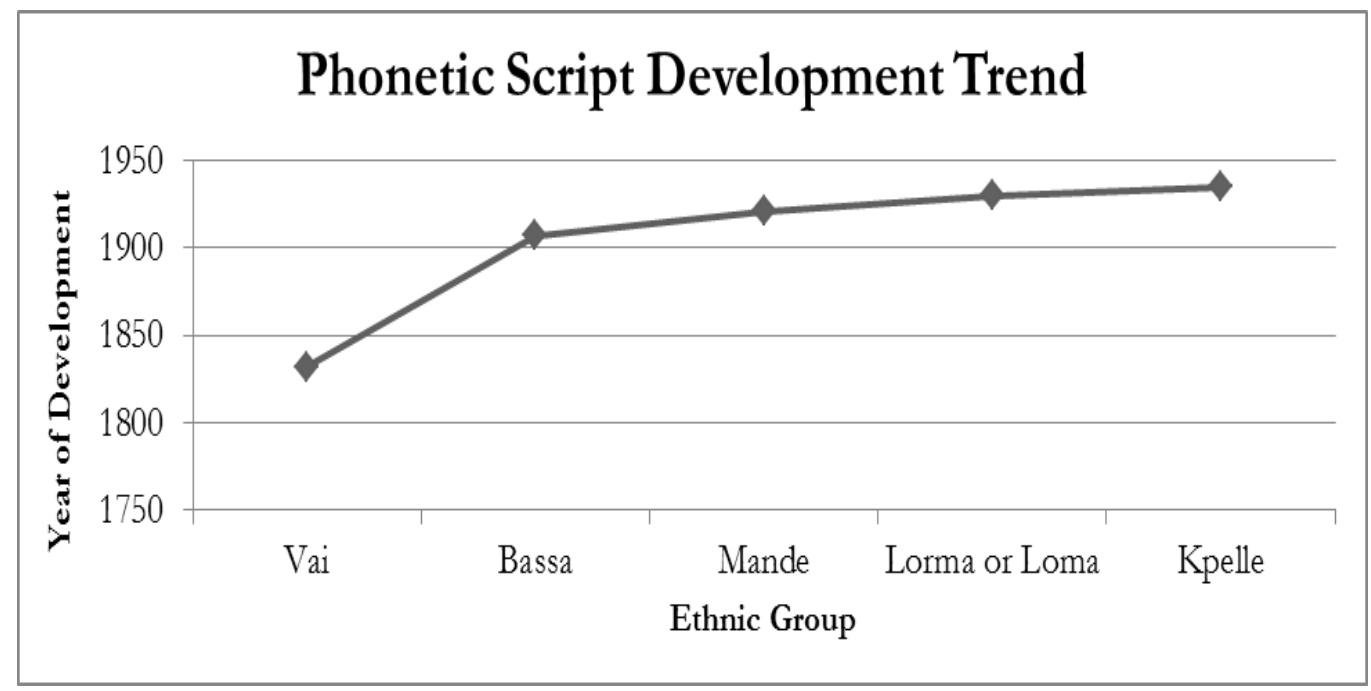

Figure 2.1: Five Liberian Phonetic Scripts

the father of structural linguistics, says, "There are no pre-existing ideas, and nothing is distinct before the appearance of language” (99).

On the other hand, Sapir suggests that language "defines experience for us by reason of its formal completeness and because of our unconscious projection of its implicit expectation into experience" (qtd. in Eastman 75). Both de Saussure and Sapir present a way of thinking about language that buttresses the core principles inherent in Schiffman's definition of linguistic culture. Schiffman indicates that knowing and understanding the linguistic culture of each society may result in great dividends for both the native speaker and the outsider in that within numerous linguistic cultures, "both the speakers and outsiders who know the culture speak of the strong bond between language and culture" (11), which allows for meaningful cooperation.

Emphasizing the strong bond between language and culture remains a pressing national challenge for health-risk communication in Liberia. Despite claims by authors of the 2007 Liberia Demography and Health Survey about the lack of "acceptable written scripts” for local Liberian languages, a Liberian weekly, The News, published a story in 2004 of a group of elementary students at a private boarding school near Monrovia "who wrote some common nouns, verbs, short sentences as well as the days of the week in...Vai scripts and phonetics" on the blackboard to publicly display their newly acquired Vai language skills. The story described the display as an "outstanding 


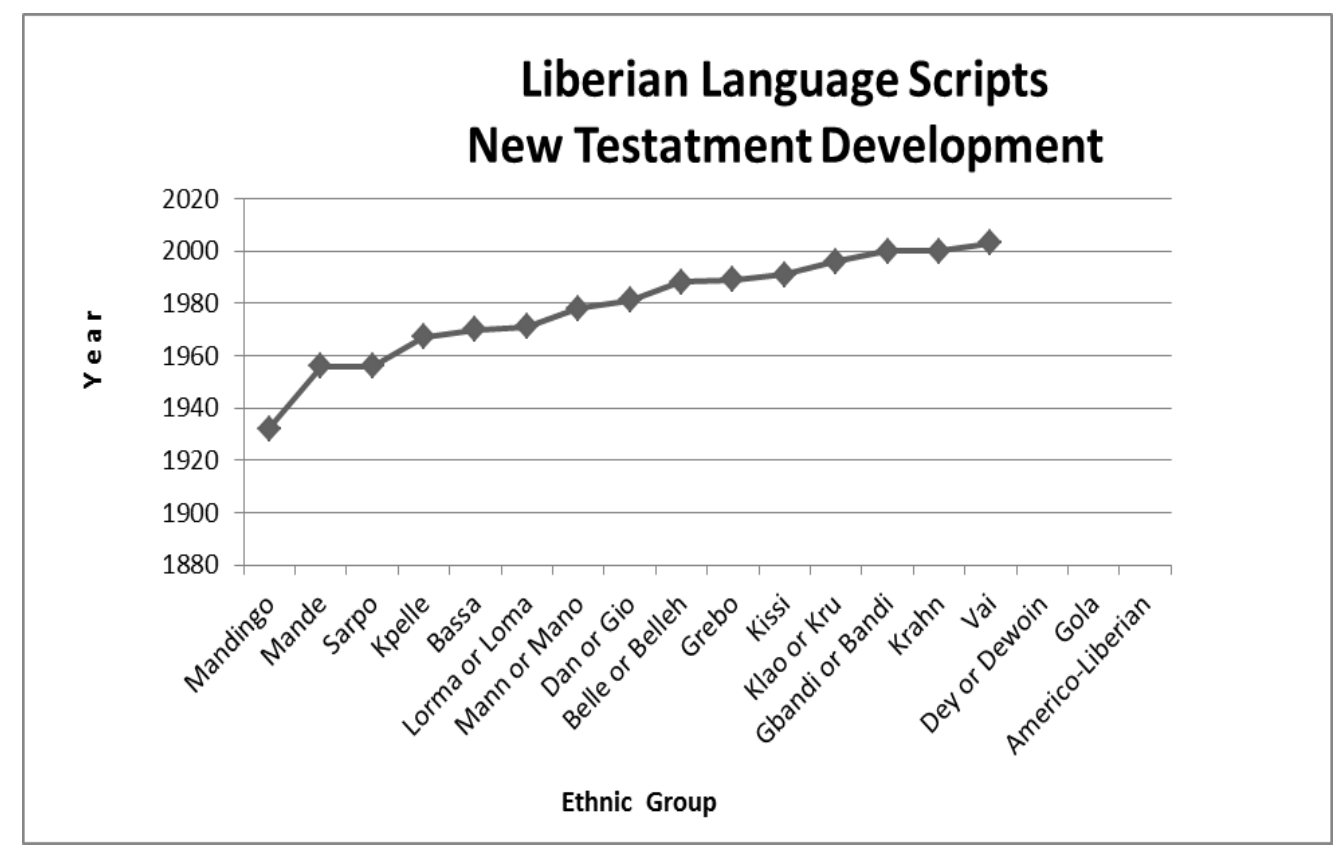

Figure 2.2: New Testament Translated into Liberian Languages

performance [that] thrilled [the] audience," and concluded that "the students proved that the Vai language has [a] magnificent, distinct and unique set of characters that have long since been in use in Grand Cape Mount Count as a means of communication” (Asumana).

The paper's excitement about a group of elementary students writing in Vai was understandable. Vai is one of 16-plus indigenous languages in Liberia; it is also one of five local languages (the others being Bassa, Kpelle, Lorma, and Mande) with phonetic scripts (see Figure 2.1). Nevertheless, these languages have yet to find their way into the national grade school, middle school, high school, and college curricula, except for private, freelance efforts of the kind described in The News.

Although Christian Bible translations (see Figure 2.2) are available in nearly all Liberian languages, the preference for English ${ }^{2}$ as the medium of instruction in Liberia has made writing in Vai and other local Liberian languages, especially in the classroom, a major news item. As a result, the languages, cultures, and traditions of the 16-plus

\footnotetext{
${ }^{2}$ The preference for English over local Liberian languages dates far back to the country's declaration of independence in 1847. The early leaders of Liberia were mostly former Black-American-slaves (Americo-Liberians), who instituted a policy of exclusion that failed to integrate the languages, cultures, and traditions of the majority Native Liberians into the governance structure of the country.
} 
indigenous ethnic groups of Liberia are complete mysteries to many Liberians. Many Liberians_-young and old, Western educated or otherwise-know little or nothing about their own languages, cultures, and traditions, and even less about those of other Liberian ethnic groups, even within the counties in which they reside, or in those counties with which they share a common geographical boundary.

The use of English for education, business, and public policy in a society in which the majority of the people can barely speak, let alone read and write, English may not be a good strategy for promoting effective communication. It may also not be a good strategy for promoting social justice and equality, given that English and other socalled dominant languages are "particularly effective in structuring inequality" in society (Tollefson 12). In 2006, only 70,000 of Liberia’s 3.8 million people (2.3 percent) spoke English as their first language (Lewis “Ethnologue.org”), but English proficiency has been a major requirement for public- and private-sector jobs in Liberia since independence in 1847 (except for jobs such as paramount chief, clan chief, and tribal governor, which require in-depth knowledge and proficiency in specific indigenous languages and cultures). The dominance of English over local Liberian languages - especially languages with functioning phonetic scripts ${ }^{3}$ developed for scholarly pursuits between 1830s and 1930s, such as Vai (1832), Bassa (1907), Mande (1921), and Kpelle and Lorma (1930s) — is a concrete example of Tollefson's notion of a dominant language effectively structuring inequality in society (4). In the 164 years since independence, little or no effort has been made to develop and use any of the 16plus indigenous Liberian languages (and dialects) for education or scholarly purposes.

There is, however, no easy solution to the problem of a dominant language structuring inequality in society. To the extent that a dominant language is learned and mastered in both written and spoken forms by all members of a given community or society, it helps to promote and foster a common method of communication for understanding and cooperation in that community or society. Conversely, where only a tiny percentage of an entire population can speak and read and write in that language, as with English in Liberia, a dominant language promotes sociocultural injustices and

\footnotetext{
${ }^{3}$ Liberia is the only country in the modern world with four indigenous language scripts developed locally.
} 
economic and political inequalities. For Liberia, though, the problem of English structuring inequality could be mitigated in two ways: (1) All five local languages with phonetic scripts could be developed and used in education, business, and public policy as regional languages in areas of the country where each language is widely spoken; 2) the two most widely spoken local languages with large numbers of native speakersBassa and Kpelle — could be developed, elevated to status of national languages, and taught in schools across Liberia.

The use of local languages in education, business, and public policy is important because as Witte, Meyer, and Martell point out, "Every group has its own lingo, its own jargon that is immediately understandable to the members of that group... [and] incomprehensible to people outside that group” (7). Every Liberian ethnic group does have its own language for effective communication, and it is a serious mistake not to exploit these language channels in the design and dissemination of health-risk messages in the country. The extent to which local Liberian languages could be developed and incorporated alongside English in school, business, and public policy to promote effective communication was the major theme of the March 2010 keynote address of Nimba County Representative Worlea-Saywah Dunah at the 14th Annual Convention of the Liberia Translation and Literacy Organization (LIBTRALO), a Christian Bible translation service.

Dunah expressed concern that English continues to be the primary language for disseminating Liberian-government policy information when "over 80\% [of Liberians] cannot understand the English language.” He said that as long as the government's message is not targeted at the over 80 percent who can't speak English well, nor read and write in English, the message "is not being disseminated at all." Dunah also said that Liberian languages and cultures which are "the root of our society continue to suffer neglect; our capacity to maintain a distinctive national identity is in shambles" (Dunah). The significance of Dunah's concerns cannot be underestimated, and these concerns have wide-reaching implications for constructing effective riskcommunication messages. While the current national literacy rate is upward of 20 percent (see CIA World Factbook), Dunah's concerns underscore the point that 
understanding the linguistic culture and communicative practices of a country may very well impact how best to adapt health-risk communication to diverse populations and cultures. For "Most ethnic minorities that are threatened with extinction," says Schiffman, "would (and do) claim that without language, their culture is dead" (8-9). Undoubtedly, language is an essential tool for human communication and social interactions, especially for cross-cultural exchanges.

Often times, as insiders, and even more with outsiders, we take for granted the important role language plays in the political, socioeconomic, and cultural life of society. According to Ricento, language is usually "something that most of us take for granted most of the time" until we discover our own inadequacies in not speaking the preferred language proficiently, or not understanding it altogether (21). Language is also susceptible to individual interpretations by outsiders of basic concepts, idioms, and dogmas, which may not accurately reflect the particular language or culture. Hence, the formal study of linguistic codes (such as lexicons or grammar) is necessary but not sufficient to our understanding of how people communicate (Schiffman 7). It takes more than the ability to speak a language to understand that language and its cultural contexts: it also takes knowing and understanding the linguistic culture of that society. For language is not simply a cultural construct; it is also something that is neither "inherited genetically from one's parents," nor "reconstituted and reconstructed by every speaker anew in every generation” (Schiffman 8).

Given the limited use of local languages in education, business, and public policy, current linguistic-cultural practices for communicating health-risk messages and public-policy information in Liberia can be characterized as one-way communication. Critical messages are often translated in chunks from English to a local language for 30-second spots on radio and television, which may not be enough to allay the fears and apprehension of the people. Neither 30-second spots on radio and television nor elongated speeches in English by public officials and health professionals will result in effective communication when the majority of the population does not speak English well or read and write in English at all. Hence, as Rep. Dunah indicates, if public information dissemination (and to a large extent health-risk messages) is not targeted at 
the level of those persons in Liberian society who can neither speak nor read and write in English proficiently, then health-risk messages and other public-policy information should be considered undeliverable.

\subsection{Diaglosia and the Territoriality of Risk Communication}

Both creating and disseminating effective health-risk messages depend largely on understanding the linguistic culture of each society. According to Eastman, “different languages imply different cultures, especially in terms of genetic relationship and genetic classification” (64). Therefore, in order to better understand a local "culture and the full description of it in a foreign language," it becomes necessary to reduce "the significant attributes of the local classification into culture-free terms” (Sturtevant 102). These significant attributes of different language variations can best be expressed through the concept of diaglosia. According to Schiffman, diaglossia is both a “community feature” that is a characteristic of a particular linguistic group (13) and "a social construct that governs the uses and functions of different [language] varieties”

(5) in society. Charles Ferguson first defined diaglossia in 1959 as a linguistic situation in which two varieties of the same language-e.g., American English versus Liberian English or Parisian French versus Haitian Creole-are functionally employed to facilitate effective communication within a speech community (379). To Paulston and Tucker, "the very essence of diaglossia has to do with the existence in the speech communities of two or more significantly discrepant speech values, one of which is a universality available vernacular variety, and the other to some degree a superposed variety, used and acquired to a greater extend in more formal contexts” (373).

Accordingly, understanding the concept of diaglossia is as important as understanding local culture and traditions in the design of effective health-risk messages targeted at members of all linguistic or speech communities. Every linguistic culture has not only cultural forms, folk belief systems, and ways of thinking about language, but also language varieties, the functions of which are to make communication clearer and more accessible within each speech community. In Liberia, for example, “Standard English” and "Simple (Liberian) English” are often used to 
target specific, local speech communities with radio and television ads and government public-policy information. No one has yet studied the effectiveness of Simple English news and ads among local populations that do not speak English fluently, and of Standard English news and ads among local populations that do speak English fluently. However, the fact that health-risk messages are conveyed in these two versions of English indicates that authorities do recognize speech communities that make up the linguistic culture of each society.

Language and culture are inherently local, and together they play a pivotal role in shaping the worldviews of local-language speakers. According to linguist Benjamin Whorf, "Every language contains terms that have come to attain cosmic scope of reference, that crystallize in themselves the basic postulates of an unformulated philosophy, in which is couched the thought of a people, a culture, a civilization, even of an era” (269). To Whorf, language has interpretative qualities and ontological values for fostering human understanding and cooperation through communication and social interactions.

This postulation, the Whorfian Hypothesis, has long drawn reactions from both critics and admirers. Many critics of the Whorfian Hypothesis have argued to the contrary that language does not influence thought. To analytic philosopher Donald Davidson, no language is untranslatable and unique enough to shape human thought and cognition to the exclusion of non-speakers of that language. Davidson argues that once the speaker of a language is capable of interpreting other languages, the speaker's first language can be interpreted by others. Nevertheless, Davidson acknowledges that language may contain a conceptual scheme, and he argues that "where conceptual schemes differ, so do languages" because the "speakers of different languages may share a conceptual scheme provided there is a way of translating one language into the other” (272).

On the other hand, Edward Sapir argues that "No two languages are ever sufficiently similar to be considered as representing the same social reality” (69), and Stuart Chase contends that "There is no one metaphysical pool of universal human thought" because "Speakers of different languages see the Cosmos differently, evaluate 
it differently, sometimes not by much, sometimes widely" (x). Faccone et al. also contend that while different linguistic cultures may have "specific language for certain ideas and concepts," it is culture that more generally produces "differentiated ways of thinking” and “'linguistically’ differentiated thought patterns” (4). And, for Sellnow et al., "The relationship of humans to the cosmos has something to do with how our relationship with nature and the spiritual world is viewed" because when communicating across cultures, "Perceptions about the nature of life, the purpose of life, and the human relationship to the cosmos contribute to an individual's world view" (43). Whether or not one believes that language can attain cosmic scope or crystallize a philosophy, the relationship between risk and language (and linguistic culture) is very clear. The characteristics of individual cultures and civilizations are essential indicators in designing and structuring risk-communication messages because they create a sense of identity and understanding of the people and the culture of each society.

Although all societies in all epochs of human history have been subjected to various kinds of risks or threats to human health, life, and property, individuals and groups have always been able to experiment with and engage in certain practices for reducing risks to themselves and the societies in which they live. Sociologists Ulrich Beck and Anthony Giddens contend that modernity is fraught with uncertainties and challenged by a series of risk factors-from the personal (e.g. jobs, homes, family, safety, and health) to the environment (e.g., air and water pollution, climate change, and toxic chemicals) - that have continued "to impact the quality of human life and happiness in un-measurable ways" (Lupton 72). These risk factors and their impacts on human society make risk communication concerned mostly with "how people deal with hazards, how risk information is processed and evaluated and how accepted information affects risk perception, evaluation and behavior" (Rohrmann Sec 2.1). Hence, communicating health-risk and related messages is more likely to be successful "when participants are seen as legitimate partners, and when people's attitudes and 'worldviews' regarding environment and technology are respected" (Rohrmann Sec $1.2)$. 
As discussed in chapter 1, risk communication is a participatory process that encompasses a wide range of people and communities (e.g., rural and urban dwellers such as farmers, traditional leaders, policymakers, scientists, and writers). Given the different interests and different risk perceptions of these communities of people, risk communicators have sought to rely on language and culture to convey risk messages and to foster social interactions and understanding, governance structures, and technological advances that are unique to each society. Hence, if the clients or participants (i.e., audiences or consumers) and the designers of risk communication do not share the same language, cultural values, and belief systems, then there is bound to be a serious disconnect in the communication process. Therefore, as burdensome as it may seem, the role of language and culture in the design and dissemination of effective health-risk messages is of fundamental importance. Hence, risk communication is territorial in the sense that every risk-communication message is directed at a specific audience for specific purposes, and located in a particular setting: government agency, industrial plant, hospital, school, farming village, community, country, etc.

The territoriality of risk communication can be seen through more traditional communication systems and cultural practices. These systems and practices "value conventions and rules as the basis for communication" and see good or effective communication as that which "successfully enacts the correct cultural conventions" (Kim and Gudykunst 50). For instance, drawing on Marxist literacy criticism, Peter Barry indicates that “A writer's social class and its prevailing 'ideology' (outlook, values, tacit assumptions, half-realised allegiances, etc.) have a major bearing on what is written by a member of that class" (158). 


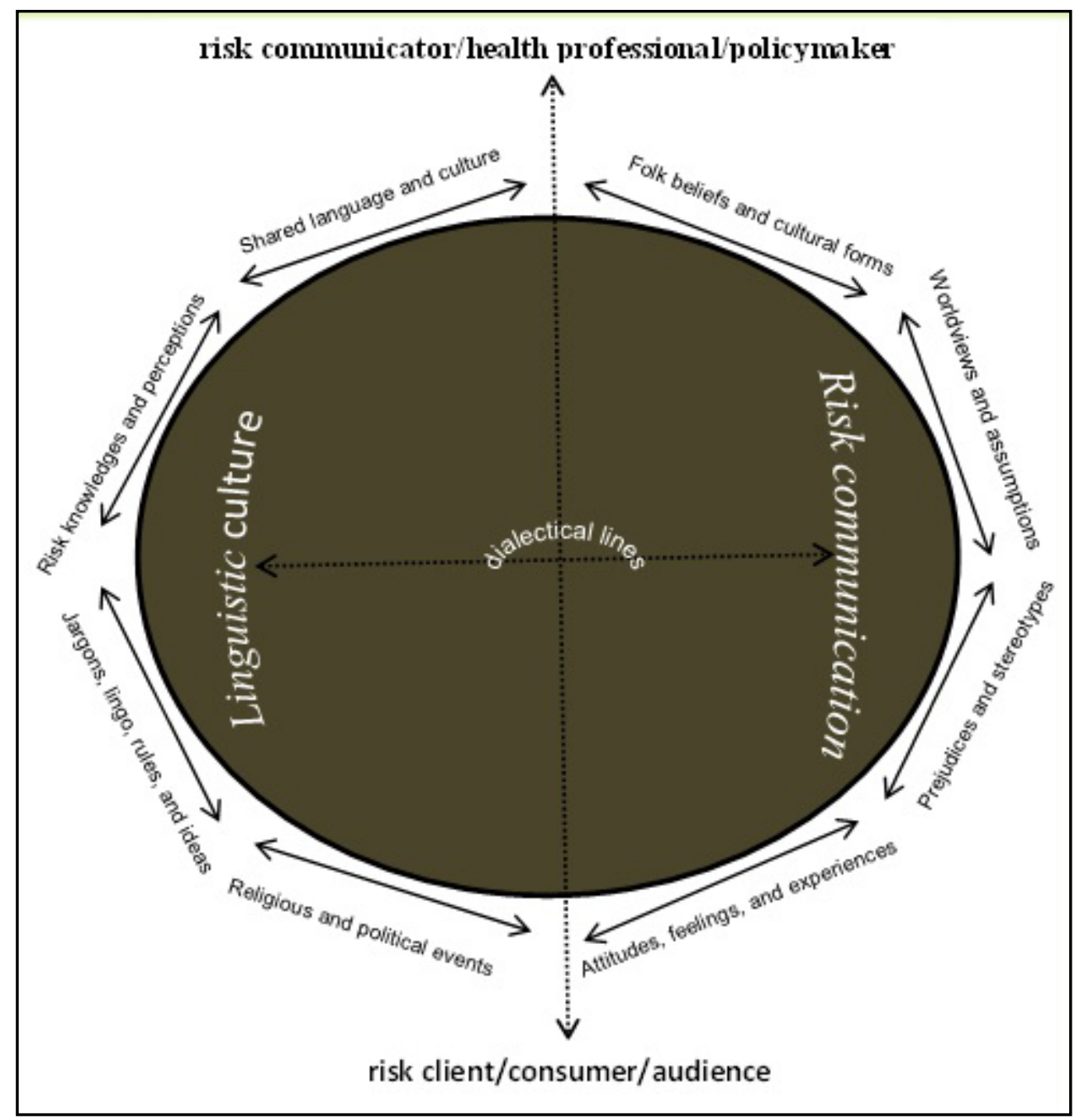

Figure 2.3: Dialectical Graph Between Linguistic Culture and Risk Communication

This appeal to social class and ideology mirrors the "governmentality theorists" approach to risk communication, which uses philosopher Michael Foucault's theory of governmentality to explore risk in the context of surveillance, discipline, and regulation of populations and associated norms. In this case, governmentality seeks to understand how government and its internal and external publics and social institutions deal with risk in individual societies, and how in the contextualization of risk responses, one ought to understand that the risk concerns of one social group in one historical era may not worry another (Lupton 25). For example, while people in early societies confronted such risks as flood, famine, or smallpox, people in modern societies are now most 
concerned with risks such as atomic weapons, nuclear waste, pollution, or HIV/AIDS. Hence, if people must accommodate to a system in which communication is valued as a means of bridging the gap between individual differences and negotiating individual realities, then belonging to a common linguistic culture is key (Winkelman 21).

\subsection{Dialectical Relationship of Risk Communication and Linguistic Culture}

As I suggested in the introduction, linguistic-cultural elements-such as folk belief systems, cultural forms, ways of thinking about language, and religio-historical circumstances (see Figure 2.3) —not only interact with one another, but also help to facilitate and sustain social communication, including risk communication. In order to effectively communicate with diverse audiences, risk communicators must show sensitivity toward cultural differences and learn the local linguistic culture, as well as seek to construct health-risk messages for specific audiences by taking into consideration such audience characteristics as "ethnicity, economic status, education, family size and status, household structure, information retrieval, language mastery, neighborhood, and technology” (Sellnow et al. 34). Hence, risk communication is "a web, a network, an interactive process for exchanging information, opinions, and values” among all participants involved in a risk-communication situation or exchange (Grabill and Simmons 425).

Risk communication and linguistic culture are two empowering tools for mitigating health risks in society. The relationship between these tools is - and should be-dialectical in that identification of risks and risky behaviors, and the means by which risk information is communicated, are part and parcel of the cultural forms, belief systems, stereotypes, and other elements of linguistic culture. In order to hybridize and indigenize health-intervention services at the local level, Hahn and Inhorn argue that local populations should not only be participants, audiences, or consumers of risk information, but they should also be producers of riskcommunication messages by way of their active involvement at every level of riskcommunication design and implementation. 
In addition, Huff and Kline suggest that in order to develop "a genuine interest in and respect for cultural difference," health professionals should develop "communication attitudes and skills that demonstrate an appreciation for and sensitivity to cultural difference" (15). They also suggest that health professionals should "begin by reading about other cultural groups, learning a new language, attending multicultural events, or spending time in communities representative of the cultural or ethnic group of interest” (15). These suggestions establish a two-way communication process that involves diverse cultural and linguistic groups with their own socioeconomic interests, belief systems, stereotypes, and perceptions about health and illness. According to a 2004 risk communication manual of the U.S. Environmental Protection Agency (EPA), two-way communication works best because "non-experts want access to information and to gain knowledge ....[while] technical experts and officials also want to learn more about non-experts' interests, values and concerns” (29-2). Bennett et al. add that two-way communication is advantageous because it usually allows all stakeholders (e.g., technical experts and participants) "to clear the air and negotiate their particular positions and help to overcome problems of anxiety and apathy" associated with their responses and reactions to various risk factors (265). Yet in order for diverse groups to confront health, safety, and environmental risks within their own and others' communities, they must learn to commit to working together, in spite of their cultural and linguistic differences. To facilitate such cooperation, risk communicators must design health-risk and related messages to appeal to diverse elements within each community, culture, or society.

According to Craig Waddell, "Risk communication is not a process whereby values, beliefs, and emotions are communicated only from the public, and technical information is communicated only from technical experts" ("Saving" 142). Instead, Waddell argues that risk communication is an interactive, two-way process that facilitates exchanges of information between technical experts and the public, who often seek to "appeal to, and engage values, beliefs and emotions" (“Saving” 142). But as Fredericks and Hodge suggest, developing "a culturally appropriate educational intervention" program hinges on "the available resources and the important cultural 
themes of the target population” (313). For inherent within any effort to communicate health risks across diverse cultures is the need to foster a dialectical relationship among those cultures. Hence, successful risk-communication is dependent upon a clear understanding that cultural beliefs and practices are germane to averting potential conflicts that could derail health-communication programs in which the local people have not been adequately involved in program design and implementation.

Fostering a dialectical relationship between risk communication and linguistic culture is not a cut-and-dried process. According to Sellnow et al., scholars in risk and crisis communication have consistently "ignored domestic multiculturalism”-i.e., the idea that people of diverse cultural and linguistic groups co-exist within any given society — when developing health-risk and related messages (47). They explain that several flawed assumptions account for this lapse: (1) well-constructed health-risk and related messages appeal to a broad, homogenous audience; (2) cultural and linguistic groups share more similarities than differences; (3) health-risk or crisis messages can be constructed based on an established pattern, and single-channel, risk-message dissemination is more effective; and (4) the use of a single spokesperson is the best way to communicate health-risk and crisis-communication messages (47). These assumptions presuppose that people of particular communities, cultures, and societies perceive and react to risk in a homogenous manner because of their close proximity and close, daily interactions. But, as stated earlier, Eastman contends that different languages infer different cultures, and Williams notes that every culture has its own shapes, purposes, and meanings.

Many cultural and linguistic groups differ greatly due to such factors as "language, perceptions about their place in society, normative beliefs and values, and world view” (Sellnow et al. 48). Understanding these factors can go a long way toward understanding “ethnic differences in health status” (Wilkenson 20) and riskcommunication practices, although previous research in risk and crisis communication has provided "little insight into the process of how multicultural publics perceive risk and crisis messages” (Sellnow et al. 34). This lack of deeper insight and understanding of the multicultural public's perception of risk messages doesn't mean that culture has 
not been a variable in risk- and crisis-communication research. According to Sellnow et al., the "inclusion of culture as a variable" in risk and crisis communication is not new. Many risk- and crisis-communication scholars have often opted to define "culture from the sender-oriented perspective” whereby "cultural groups are viewed as we-they or usthem” (34). This poses some challenges for promoting a dialectical relationship between risk communication and linguistic culture, but these challenges are not overwhelming.

How, why, where, and to whom risk information is communicated have become the new impetus for finding effective ways of communicating risk to diverse populations. The number of citizen groups seeking to be heard at the decision-making table in risk-communication and public-policy matters has rapidly increased in industrialized nations (Bennett et al. 57). The nature of modern society has also led to increased awareness, fear, and sensitivity about risks and risk issues. Hence, a growing emphasis in risk-communication research and practice has been on how to prevent risk information from being "couched in a language that is not readily understood by the receptors of the message" (Bennett et al. 249). These developments speak to a continuing need for a dialectical relationship between risk communication and linguistic culture.

As Witte, Meyer, and Martell note, "Even the best [health-risk and related] messages are not likely to be effective if inappropriate channels are selected for dissemination” (133). Effective risk communication takes place when information is disseminated through "a community-compatible language or form" (EPA 29.5). The symbolic value of using a local language in creating and disseminating health-risk and related messages cannot be overemphasized. As Barry notes in his analysis of de Saussure's structural theory of language, "Language constitutes our world, it doesn't just record it or label it” (43). It is through language that risk messages are crafted and disseminated, and it is language that fosters a dialectical relationship between risk communication and linguistic culture, based on the cultural forms and folk belief systems of individual cultures and societies. 


\section{Chapter 3: Research Methods}

\subsection{Purpose of the Study}

The purpose of this study was to assess malaria-treatment-and-control programs (MTCPs) in Liberia to find out what effects—if any-local culture and traditions have on the process of communicating health risks to diverse populations. The very idea of communicating across diverse communities, cultures, societies, and international borders presents multiple complexities and challenges for social interaction and understanding, such as language and culture, political climate, health and environmental regulations, and religious and non-religious beliefs. These are unique societal characteristics that also manifest themselves in how each community or society defines, perceives, and communicates risks. Hence, in this study, I also examine cultural and social factors, such as language and folk belief systems that have the potential to promote or hinder incorporation of local culture and traditions in the design and implementation of MTCPs and health-risk messages. I also attempt to answer specific questions about how cultural adaptations affect programmatic outcomes of MTCPs and how MTCPs that adapt health-risk communication to a particular ethnic group might be most effective. I also evaluate possible inaccuracies in MTCP program reports about goals and levels of cultural adaptation and about how people's cultural values, traditions, and mores become integral parts of program design and implementation.

\subsection{Research Methodology and Design}

In this study, I employ qualitative research methods (e.g., survey questionnaires and qualitative interviewing) for data collection, and rhetorical theory for data analysis. I originally intended to collect and analyze only existing policy documents from selected MTCPs in Liberia and draw conclusions therefrom on how to adapt health-risk communication to the specific cultural contexts of diverse populations. However, after much thought, and in consultation with my advisor and some professional colleagues, I decided that it would be highly desirable to include field research in Liberia that targeted both the designers/implementers and beneficiaries of MTCPs. Hence, I 
collected and analyzed three sets of data: (1) existing-policy documents on MTCPs in Liberia (e.g., from the President's Malaria Initiative [PMI] and the Liberian National Malaria Policy), (2) survey questionnaires, and (3) field interviews. I collected data for the survey questionnaires and interviews during field research in Liberia from February to May 2011.

According to Warren and Karner, the two most common methods of data collection in qualitative research are field research (participant observation) and qualitative interviewing (10). They note that although both data-collection methods involve direct interactions with participants in a field setting, qualitative interviewing stands out for its structural nature, wherein the researcher asks research participants a set of specific questions using a tape recorder or other recording device, as opposed to field observations and note-taking only (118). Qualitative interviewing facilitates social interactions between the researcher and research participants intended "to locate valid and reliable information, with the interviewer directing the questions and the interviewee answering them as truthfully as possible,” in a display of "partnership and dialogue as they construct memory, meaning, and experience together” (Madison 25). Hence, qualitative interviewing became the preferred method of data collection, involving the recipients of insecticide-treated bed nets and related MTCP services, such as indoor residual spraying and Artemisinin-based combination therapy.

On the other hand, survey questionnaires - which provide an opportunity for self-expression and self-evaluation without the presence of the researcher-became my preferred method of data collection involving designers, implementers, and administrators of MTCPs. This method of data collection is also less expensive and less time-consuming to both the researcher and the research participants, although response rates are often low in the absence of extensive follow-ups. Collectively, though, the various qualitative research methods provide individual tools for understanding how and why people in certain settings (e.g., community, town, village, classroom, workplace, or culture) live or act in the way they do, or how and why a certain event or phenomenon impacts a group of people or community in the manner that it does (Denzin and Lincoln 21). In particular, undertaking qualitative, field-based research 
involves an attempt by the researcher to unravel a social phenomenon of interest that holds a broader meaning or implication for society: at least a "social phenomenon from the actors' perspectives through participation in the life of those actors" (qtd. Firestone 17). Whenever one engages in qualitative research one must not only "strive to become a good writer; but...also strive to become an attentive researcher” (Warren and Karner 10). Attentiveness is key to discovering the hidden meanings of a phenomenon, especially the world of the lived experiences of individuals, since this is "where individual belief and action intersect” (Denzin and Lincoln 8). The findings of qualitative research are also "not independent of the methods used to produce those findings, or the standpoints and perspectives of the researcher" (Warren and Karner x). Hence, for this study, the insights of designers, implementers, and administrators of MTCP and the lived experiences of beneficiaries of MTCPs became crucial to understanding how to adapt health-risk communication to specific populations.

I use rhetorical criticism to analyze most of the data in this study. Not only that the goal of rhetorical criticism (i.e., the rhetorical critic) is to advance knowledge about human communication and related public messages, but also to help us understand controversy or interesting messages "within a larger framework of knowledge about human communication and cultural persuasion” (Pierce 31). Such a goal empowers the rhetorical critic to investigate various aspects of human communication that took place within a historical or contemporary period by people of society, especially policy makers (Pierce 47).

According to Sonja Foss, rhetorical criticism is also "a qualitative research method that is designed for the systematic investigation and explanation of symbolic acts and artifacts for the purpose of understanding rhetorical processes” (6). Foss says that of the different methods of rhetorical criticisms-e.g., metaphor, cluster, or pentadic criticism—generative criticism uses a "process...much like the groundedtheory approach to analyzing data" (405). According to Ownby et al., grounded theory aims to generate theory from the data, not from outside of it (48-59), especially when little is known about a particular subject, topic, or phenomenon, and when existing theories proved inadequate to explain such a subject, topic, or phenomenon (Creswell 
53). However, because the process of how to communicate health-risk messages to diverse populations is grounded more in theories of rhetoric and technical communication than anything else, rhetorical criticism became the preferred method for analyzing the research data underlining this study.

Rhetorical criticism is an analytic tool for understanding human activities within a specific context or rhetorical situation. Like all other forms of criticism, rhetorical criticism is a humanistic activity that aims to understand people's "acts and creations" (Black 9). It has "no relationship with its subject other than to account for how that subject works" (Black 18), especially within the cultural and linguistic contexts of individual societies. Because "there is no way of experiencing the 'real relations' of a particular society outside of its cultural and ideological categories (Hall 245), rhetorical criticism seeks to analyze various artifacts of society in order to understand their relations to other things within that society. Given that an artifact is a "tangible, retrievable trace or recording of the original messages” (Pierce 47), individual artifacts are usually interpreted "against a backdrop of shared understandings, practices, language, and so forth" (Schwandt 197).

Pierce also suggests that in order to understand "how messages in human communication work," it becomes almost a matter of second nature that many of us engage in "informal rhetorical criticism (i.e., trying to figure out why certain messages seem to work widely well, while others seem to bomb)" (9). This informal attempt at rhetorical criticism has given rise to the formal study and application of rhetorical criticism. Hence, the province of rhetorical criticism is the analysis of various historical and contemporary artifacts in order to understand how these artifacts and related messages are constructed and communicated in society, given individual preferences and diversities in culture, language, learning styles, and communication patterns. Because "to engage in any communication, is to participate in a community" (Miller 617), rhetorical criticism usually aims to engender understanding of an artifact through careful analysis of its features in light of its rhetorical situation and the audience, environment, or community in which it exists. 


\subsection{Site of the Study}

My research study was conducted in Liberia, specifically in Monrovia (the national capital); in the city of Buchanan, Grand Bassa County; and in neighboring border towns in Rivercess County. As home to various government ministries and agencies, and national offices of international NGOs and other aid agencies (e.g., the Ministry of Health and Social Welfare, the National Malaria Control Program [NMCP], WHO, Global Fund, and PMI), Monrovia became the ideal site from which to reach designers, implementers, and administrators of MTCPs. In order to minimize travel logistics and ease language barriers, I targeted Buchanan and its environs for the qualitative-interview portions of the study. Buchanan, one of the largest cities in Liberia, is located about 88 miles outside Monrovia, which makes it more accessible by road than other parts of Liberia, where the country's roads have been significantly degraded by the 14-year civil war. In addition to its relative ease of access, the main language in Grand Bassa and Rivercess is Bassa, a language which I speak. Hence, although some language translators aided in the data collection process, the need for such translators was limited. Nevertheless, since English is the official language of Liberia, and Bassa-language scripts are not widely used in education or research, I conducted the interviews both in Bassa and English (including Liberian Pidgin English).

\subsection{Participants and Sample}

I initially hoped to involve 100 participants (20 for survey questionnaires and 80 for field interviews), but 105 participants responded. The additional five participants (1 for the questionnaires and 4 for interviews) were not completely unexpected, however, in that about 50 survey questionnaires were hand-delivered or emailed to potential participants, and in some communities, up to 10 participants were interviewed one after the other on a given day.

Two categories of participants were selected for this study: (1) designers and implementers of MTCPs (i.e., program administrators and staffers from international NGOs, other aid agencies, and government health services) and (2) beneficiaries of MTCP services (i.e., recipients of bed nets, indoor residual spraying, etc.). The first 
category of participants was recruited from the Monrovia and Buchanan offices of local and international NGOs and other aid agencies as well as from government agencies involved with MTCPs in Liberia. In order to make initial contact with survey participants, I obtained a contact list from the National Malaria Control Program containing the names, phone numbers, and email addresses of the program's participating partners. Because the questionnaire was meant to be completed anonymously, I did not record the names and contact information of participants, but used the list to send out the questionnaire to everyone in each office (employee or consultant) with a solicitation letter that explained the purpose of the study and the procedure for completing the questionnaire.

I asked this category of participants to provide demographic information on agency affiliation, length of service, and job function as well as information on the design, implementation, and outcomes of the specific MTCP with which they were affiliated. Copies of blank questionnaires were emailed or hand-delivered to each participant by myself or a designee. Each participant had up to two weeks to complete and return the questionnaires, with the option of attaching supporting documents, such as annual reports or strategy papers. Participants also had the option of emailing the completed questionnaires to me or asking me or my designee to pick up their completed questionnaires at designated offices in Monrovia and Buchanan. In order to avoid duplication and to keep track of the number of participants from each MTCP office, I coded each questionnaire prior to distribution. For example, the code NG\#0001UN stood for the first questionnaire distributed at a local UN-affiliated agency, such as UNDP. The anonymity and confidentiality of each participant was protected in that the codes were intended to track how many questionnaires were sent to each participating agency rather than to track participants themselves. Hence, since participation in the study was voluntary, the first category of participants was self-selected, and identified on the questionnaire only as program administrator, staffer, designer, or implementer. 
The second category of participants was identified and recruited from communities within Buchanan and its environs-including Kpelle Town, Dark Forest Field, Sanwein, Vai Town, Zinc Camp, Sugarcane Farm, Sawmill, and Gbediah Town (Rivercess County) — where bed nets have been distributed and indoor insecticide spraying has taken place under MTCPs. The participants were chosen at random (e.g., based on odd numbers) from a list of beneficiaries of MTCPs in the Buchanan area provided by the National Malaria Control Program. The list ensured that participants were actual beneficiaries of bed nets and related MTCP services and, hence, were knowledgeable about the subject of the study. Based on the list and the help of a few informants and gatekeepers, I made initial contacts with the participants in their homes or offices to arrange times for the field interviews.

On the day of each interview, I informed each participant about the purpose of the research and waited for each participant to complete a consent form prior to starting the interview. I then asked the participants demographic questions (about their age group, gender, community of residence, and length of residence), and questions about their knowledge of malaria, the name by which malaria is called in their local language or culture, their use of bed nets and related items, and methods of communicating health risks in their ethnic group, culture, or language. The specific place and time for the interviews depended on the place and time most convenient for each participant. Most of the interviews were conducted in the late afternoon and sometimes stretched into the early evenings, which necessitated the use of lanterns or candles due to the lack of electricity.

Although I may have observed an entire family unit, household, or village (including minors) during field interviews, only persons 18 years of age or older participated in the interviews. With the aid of a Bassa-language interpreter, I interviewed in Bassa participants who did not speak fluent English. As a result of the civil war, most of Liberia currently lacks electricity, pipe-borne water, and public transportation; hence, each participant-observation and interview took an average of 1-2 days, depending on travel logistics, meeting times, and weather conditions. 
Participants in this category ranged in age from 18 to 74, and I initially targeted 20 traditional leaders or elders, 20 heads of household, 10 mothers (30 and up), and 30 young adults (ages 18-27), 15 each male and female (see chapter 5 for actual number of participants from each category). Traditional leaders were targeted for their knowledge of the local culture and traditions, and mothers were targeted for their knowledge of and experience with using bed nets to protect children and infants. Heads of household were targeted for their knowledge of bed nets and insecticide use in the home, and young adults were targeted to provide the young people's perspectives on bed nets, insecticide, and other antimalarial treatments among young people. Many participants in this category, especially traditional leaders, had not attended Western-style schools and did not speak English well, nor did they read and write in English. With their consent, I used a digital camera and a video-camera to record images of some of the participants in this category. To ensure confidentiality and anonymity, I did not identify the participants in these images.

\subsection{Participation Criterion}

The main criterion for participation in this study was affiliation with MTCPs in Liberia: (1) as program designers and/or implementers, staff, or administrators or (2) as beneficiaries or recipients of bed nets, indoor residual spraying, and other services provided by MTCPs. Participation was entirely voluntary.

\subsection{Data Gathering Instruments and Research Questions}

The data-collection instruments for this study included survey questionnaires, field interviews, and participant observation. A tape-recorder, camcorder, and digital camera were also used to capture audio and visual recordings of the interviews and related field observations. 


\subsubsection{Research Questions}

1) Why have local and international efforts in malaria-treatment-andcontrol not yielded the desired results of eradicating malaria in Liberia and other African countries?

2) What effects do local Liberian culture and traditions have on the process of communicating health risks to affected populations?

3) To what extent do the design and implementation of malaria-treatment programs impact malaria-treatment outcomes in Liberia?

4) How do constraints—such as lack of adequately trained staff, limited funding, cultural resistance, corruption, and governmental bureaucracy_-impact health-risk communication in Liberia?

\subsection{Data Analysis}

This study employs rhetorical theory for data analysis, especially generative criticism, pentadic criticism, and the classical rhetorical concepts of logos, pathos, and ethos. Because "rhetoric is the art, practice, and study of human communication" (Lunsford), it is difficult to understand the process of how best to communicate healthrisk messages to diverse cultural populations without the help of rhetorical theory. The generative method of rhetorical criticism (see Section 3.2) provides an opportunity to generate new insights and meanings about rhetorical artifacts. It also provides dataselection and coding criteria, such as intensity and frequency (Foss 389), that fit well with my data sets. Intensity refers to the most important feature of an artifact targeted for analysis, and frequency refers to the number of times an important feature occurs during analysis (Foss 389). Generative criticism is used to "generate units of analysis or an explanation” for an artifact (see Piece, Section 3.2) when the critic desires to capture significant aspects of the artifact without the structural constraints imposed by other methods of rhetorical criticism (Foss 387).

According to Foss, generative criticism analyzes an artifact through a nine-step process: (1) encounter a serious artifact; (2) code the artifact in general; (3) search for an explanation; (4) create an explanatory schema; (5) formulate a research question; 6) 
code the artifact in detail; (7) search the literature; (8) frame the study, and (9) write the essay or research report (387). Through these steps, generative criticism facilitates an opportunity for evaluating and analyzing an artifact in order to understand how the artifact's various features convey specific messages. Basically, the specific features of an artifact is examined, categorized, and interpreted to derive conclusions about the meanings and messages each feature conveys.

Pentadic criticism is structured around Kenneth Burke’s dramatic pentad (act, agent, agency, scene, and purpose). As in journalistic practice, it seeks to answer the basic questions of what, who, how, where, and why about an artifact, although the "power" of pentadic criticism is "inextricably linked to the concept of 'ratio"” (Kneupper 132). The ratio refers to the dominant element of the five terms of the pentad during analysis of an artifact and its relationship to the other terms or elements (Foss 359-362). According to Kneupper, the pentad is a "useful tool for discourse adaptation" and serves "a heuristic function in adapting discourse to particular audiences" (134). Foss states that pentadic criticism is suited for analyzing "virtually any artifact” (357), and explains that pentadic criticism is a four-step process, including (1) selecting an artifact; (2) analyzing the artifact; (3) formulating a research question; and (4) wring the essay" (357). Because pentadic criticism assumes that "humans develop and present messages in much the same way that a play is presented” (Foss 356), one must first identify the five terms or elements of the pentad in order to begin analyzing an artifact.

Aristotle saw rhetoric as the art of persuasion, and argued that that a speaker's ability to persuade depended on three rhetorical appeals: logos, pathos, and ethos. Logos is an appeal to reason or logic (i.e., through enthymemes, statistics, analogies, or experiments). Pathos is an appeal to the emotions of the audience, and ethos is an appeal to the credibility of the speaker or writer. These three appeals are essential to conveying meanings and soliciting action across individuals and cultures.

Hence, in this study, generative criticism is used to analyze the interview data and the survey-questionnaire data. Both sets of data specifically deal with issues of incorporating culture and traditions into the design and implementation of MTCPs, and with the creation and dissemination of health-risk messages adapted to the cultural 
contexts of diverse populations, for which there has been no prior research in Liberia. I also use pentadic criticism to analyze existing policy documents about MTCPs in Liberia, such as the U.S. President's Malaria Initiative. Rhetorical devices such as logos, pathos, and ethos are used concurrently with both analytic methods of criticism in order to clarify key points and invoke certain actions.

Like any qualitative research method, rhetorical criticism is saddled with the question of validity. It is an interpretative process in which the critic or interpreter both “objectifies...that which is to be interpreted" and "remains unaffected by and external to the interpretative process” (Schwandt 194). Gerry Philipsen suggests that when we communicate, we produce messages in order to "create an intended meaning," which is not to say that "intended meanings are always interpreted as the sender would have them to be understood” (258-59). Hence, rhetorical criticism is about meaning-making through interpretations of artifacts or social events. Finding “meaning in an action” does require that one interpret the social event "in a particular way what the actors are doing" because both interpretation and understanding are "differentially represented” processes (Schwandt 191). For one can never fully understand a particular social event or action (i.e., friendship or cooking) without first grasping "the meanings that constitute that [event or] action” (Schwandt 191).

According to Edwin Black, criticism on the whole, including rhetorical criticism, sits "near the indeterminate, contingent, personal end of the methodological scale” (xi). And, particularly so that every "piece of qualitative research is very much influenced by the researcher's individual attributes and perspectives” (Wainwright 202), Black argues that the critic is "the sole instrument of observations" (xi) in any form of criticism. Black also indicates that "there are no instruments that mediate the engagement between the critic and his subject” (xi). However, while the critic is the sole instrument of observation and analysis in criticism, the critic does not operate in isolation of a public audience. Black contends that "The critic does address a public and he thereby incurs public responsibilities” (xi). While interpretations may be a personal undertaking, meaning is usually "negotiated mutually in the act of interpretation” (Schwandt 195) so that others may learn and understand what is being interpreted. For 
instance, when a critic writes, “The critic's public should, in principle, be able to verify for itself that the critical object can be apprehended as the critic proposes without offending reasons" (Black xi). As we communicate, we also "crave for meaning-a contextual meaning," that our respective audiences will relate to, understand, and act upon. Meaning is also amenable to a systematic study, such as rhetorical criticism, because "If the meanings and patterns of communicative activity vary cross-culturally, then they must be discovered in particular cases, and not assumed" as part of individual interpretations and explanations in human communication (Philipsen 260). Communicating health risk across cultures also requires interpretations, meaning formulations, and understanding, especially so that we exist in the world through meanings we create and understanding we project about ourselves and our environments.

\subsection{Ethical Considerations}

While constructing the initial research proposal, I completed all the required Collaborative Institutional Training Initiative (CITI) modules on protection of human subjects, as required by Michigan Technological University. Then, to protect the human participants of this study, Michigan Tech’s Institutional Review Broad (IRB) reviewed and approved my research proposal before I contacted participants. After I arrived in Liberia, I explained the purpose of my research to prospective participants and asked them to sign an informed-consent form. I assured every participant that he or she had the right to withdraw from the research at any time, without penalty. This was especially important during the interviews where participants might feel uncomfortable or upset because of the sensitivity of the topic. I assured participants that their responses would be kept confidential throughout the entire study and thereafter, until all data was destroyed.

Because of public perception that the 14-year civil war (1989-2003) in Liberia may have resulted from ethnic and religious conflicts, Michigan Tech’s IRB reviewers raised two questions about the safety of participants: (1) "Can you provide more information regarding details of ethnic and religious tensions in Liberia that could result 
in reprisals if the photo-movie or name of the individual was presented in other contexts?" and (2) If the participants were "identified by photo, location code...and by their responses to the demographic questions, [was I] introducing a risk not otherwise apparent in their daily life"?

These questions raised legitimate concerns about the safety of participants. However, I responded that as someone born and raised in Liberia, I was not aware of any ethnic or religious tensions in Liberia that could result in reprisals for participants in this study. Although the civil war was generally portrayed by politicians and the international press as an ethnic conflict, Liberia has really not experienced any major ethnic conflict in its more than 160-year history. Liberia comprises 16 major ethnic groups, and during the civil war, only some members of four ethnic groups (Gio and Mano versus Krahn and Mandingo) targeted one another on a limited scale; however, these conflicts were mostly political rather than ethnic in nature. The Gio and Mano ethnic groups and (some Mandingo people) live mainly in Nimba County (the equivalent of a state in the United States) and share a common border with the Krahn ethnic group of mainly Grand Gedeh County. Prior to the civil war, there had not been any ethnic conflicts between the Krahns and their Gio and Mano neighbors. In recent Liberian history, members of these ethnic groups have not attacked one another since the civil war ended. The Mandingo ethnic group—scattered about mainly in the counties of Nimba, Bong, Lofa, Bomi, and Rivercess - was not at war with the Gio and Mano ethnic groups before or after the civil war.

On the religion question, there have not been any religious tensions in Liberia except for a February 2010 incident in Voinjama, Lofa County where a few churches and mosques were burned down as a result of a dispute between a group of Muslim and Christian youths over the mysterious death of a young Muslim girl. Otherwise, Christians, Muslims, and traditional religious practitioners have coexisted peacefully in Liberia since the country became a nation-state in 1847. During the civil war, Christian and Muslim leaders formed the Interfaith Mediation Council and worked together to end the war and bring peace to Liberia. 
More importantly, there is no history of ethnic or religious tensions in the areas targeted for my research. Grand Bassa and Rivercess Counties are mostly populated by the Bassa, Kru, Americo-Liberian, and other ethnic groups, but Bassa remains the predominant ethnic group and language. Hence, given the history of the peaceful coexistence of people in the areas targeted for my research, I did not believe that presenting the photo-movie of participants in other contexts (i.e., scholarly presentations and publications) would introduce a risk to the participants not otherwise apparent in their daily lives. Nonetheless, I took care throughout my research and dissertation writing period - and will take care in future scholarly publications - to avoid or minimize any risks to the participants, such as risks related to the use of their photo, location code, and responses to demographic questions. I also did not foresee reprisals of any kind against the participants as a result of their voluntary participation in the research. During my field research in Buchanan, the majority of the participants came mostly from the Bassa, Mandingo, and Kru ethnic groups, and no ethnic tensions exists among these people. 


\section{Chapter 4: PMI-Liberia and the Malaria Challenge: A Pentadic Analysis}

\subsection{Introduction}

Although any number of theoretical choices—discourse analysis, textual criticism, or rhetorical criticism—can be used for analyzing written or textual documents such as manuscripts and policy documents, I chose to use pentadic criticism to analyze the policy document discussed in this chapter. Dann Pierce argues that "if we knew perfectly how messages in human communication work, we would have little reason to do rhetorical criticism of any type” (95), including pentadic criticism. He argues that people in "human culture (speakers, sign painters, singers, ad writers, designers, legislators, politicians, business execs, etc.) still make messages using only their accumulated experiences (trial and error) as a guide” (Pierce 95), and that selecting the method of one's analysis is "an object or goal that has many different destinations" (110). He, however, suggests that whatever method of criticism one finally takes, the ultimate goal should be to "build a new set of method steps, to borrow and revamp a set of existing method steps, or to combine two different sets of analysis steps as a method" (110).

In this chapter, I use pentadic criticism to analyze the 2008 President's Malaria Initiative’s (PMI) Malaria Operational Plan (MOP) for Liberia. A MOP is a comprehensive, malaria-treatment-and-control plan of action designed by the United States Agency for International Development (USAID) and the U.S. Centers for Disease Control and Prevention (CDC) on an annual basis to guide each year's PMI goals and activities in PMI-supported countries, such as Liberia.

Whereas discourse analysis and pentadic criticism—and rhetorical criticism in general-are concerned with understanding the meanings inherent in human communication and social structures (i.e., the rhetorical artifact) in terms of the strategies people employ and the choices they make to communicate, pentadic criticism stands out as the ideal method for analyzing the 2008 MOP. Pentadic criticism is an outgrowth of Kenneth Burke’s pentad, which uses such dramatic elements as act, agent, agency, scene, and purpose to present data-and the interpretation of it - in a more 
visually appealing way than discourse analysis or textual criticism, which is mostly concerned with analyzing biblical and related sacred texts. Pentadic criticism also provides a more methodical way to expand and/or generalize about a rhetorical artifact beyond its beginning background or scenario. This expansion or generalization is made possible through Burke's circumference of scene, for which he argues that the "choice of circumference of the scene in terms of which a given act is to be located will have a corresponding effect upon the interpretation of the act itself” (Grammar 77). Like , Searle's deep background and local background (see chapter 1, Section 1.1), Burke sees the scene as the most important aspect of the pentad. He argues not only that one may place the scene in contexts of varying scope when "defining by location” (Grammar 77), he also states that "contracting and expanding of [the] scene is rooted in the very nature of linguistic placement," whereby the selection of the circumference from among a "range is in itself an act ... with the definition or interpretation of the act taking shape accordingly” (84). The 2008 MOP, the rhetorical artifact being analyzed in this chapter, provides such an opportunity for expansion of the scene by not only the “very nature of its linguistic placement," but also by the very nature of the topology of its geographic location, and the social actors involved with designing and implementing the 2008 MOP.

Pierce not only notes that language use over a protracted of time can create "an inherited worldview" (13), but also that the physical world in which we live "will only yield to certain kinds of influence” (Pierce 14), even as we create and use messages to communicate with one another. He says that any message is rhetorical as long as it is capable of producing or encouraging change, even if the creator of the message did not contemplate such a change (11). Pierce argues that if the general goal of the rhetorical critic is "to advance knowledge about human communication that reaches audiences with public messages," then the particular goal of the rhetorical critic must be "to help us understand the controversy or interesting message(s) at hand" and "also to see where that understanding fits within a larger framework of knowledge about human communication and cultural persuasion (Pierce 31). However, when investigating cultural communication, Pierce suggests, the rhetorical critic “must seek 
times, places, people, and occasions when someone was trying to change someone else by using a type of message that would reach a large audience "(Pierce 47)

The 2008 MOP presents both an opportunity for changing lives and for expanding the circumference and generalizing about the background of scene (i.e., Liberia). This means that whatever goals embedded in and successes achieved through implementation of the 2008 MOP, such outcomes can be extended or reproduced in other communities, countries, and geographical regions by simply expanding the circumference of the scene in Burkean terminology.

Because the PMI has operated in Liberia since 2008, Liberia has MOPs for 2008, 2009, 2010, and 2011. I chose to analyze the 2008 MOP (see pmi.gov) for its significance as the first MOP and the one on which all subsequent MOPs for Liberia are based. This MOP also captures the state of malaria and malaria-treatment-and-control activities in Liberia prior to the involvement of the PMI. Furthermore, because the annual MOPs embody the operational details of each year's PMI activities in PMIsupported countries, the 2008 MOP and PMI-Liberia are used interchangeably throughout this chapter, except where the "2008 MOP” functions as a reference.

In analyzing the 2008 Liberian MOP, I look at the extent to which the PMI and other international and local malaria-treatment-and-control programs (MTCPs) operating in Liberia incorporate_ or might incorporate-local culture and traditions in program design and implementation. I also analyze this MOP as a representative sample of an existing-policy document (one of three data sets underpinning research for my dissertation, including survey-questionnaire data and field- or qualitative-interview data). The 2008 MOP also provides an example of a program that was developed on a Western model and implemented in Liberia pretty much "off the shelf" and was only in the narrowest sense adapted to a particular audience, such as a Liberian ethnic group or community. Based on my examination of existing-policy documents for the PMI, the Liberian National Malaria Control Program (NMCP), the 2009-2013 National Malaria Strategic Plan (NMSP) for Liberia, and annual reports from related organizations, I found that there is no significant difference between the design, focus, and 
implementation goals of the PMI and these other programs. This conclusion is supported by my field research in Liberia.

Both the PMI and the NMCP — which designs and implements national policy for malaria control in Liberia—are structured on Western models that prioritize in their malaria-treatment-and-control strategies the use of Artemisinin-based combination therapy (ACT), intermittent preventive treatment for malaria in pregnancy (IPTp), insecticide-treated bed nets (ITNs), and indoor residual spraying (IRS) with insecticides. All four methods (ACT, IPTp, ITNs, and IRS) are approved and recommended for use across the world by the World Health Organization (WHO) through its relations with nations and in its publication, WHO Malaria Treatment Guidelines, 2nd edition. Both the PMI and the NMCP also get most of their funding for program design and implementation from the U.S. government and/or private sources, such as the Global Fund to Fight AIDS, TB, and Malaria (GFATM or Global Fund), the Clinton Foundation, and the United Nations Development Program (UNDP). This is why prior to 2008, the Global Fund, the United Nations Children's Fund (UNICEF), and WHO provided the bulk of the funding for bed nets, ACTs, and related services in Liberia. Despite these efforts, malaria continues to ravage the country to such an extent that the entire population of 3.8 million people, (including 565,000 children under five years, and 188,000 pregnant women) is at risk of contracting malaria (“2008 MOP” 5).

As part of this analysis, I also examine the basic services provided to the local population by PMI-Liberia, especially the use of bed nets and indoor residual spraying. A breakdown of these services reflects three broad categories: (1) preventive services; (2) case management services, and (3) technical services. Each category is then evaluated in terms of how PMI-Liberia and other local and international agencies are confronting the social, economic, political, and technical realities of a post-conflict nation grappling with malaria treatment, control, and prevention with limited resources and technical capacity. With a focus on the process of adapting health-risk communication to the specific cultural contexts of diverse populations, I also examine these services and their design and implementation strategies in relation to the extent to 
which the "local setting" (i.e., local peoples and their cultures and traditions) is meaningfully incorporated into the design and implementation of these MTCPs.

\subsection{Defining the Artifact}

In pentadic criticism and other methods of rhetorical criticism, the symbols or objects of analysis (e.g., a policy document, a speech, a movie, or an architectural design) are called "symbolic acts" or "artifacts” (Foss 6). The PMI's 2008 MOP for Liberia is the artifact of study in this analysis. On June 30, 2005, during a speech at the Freer Gallery in Washington, D.C., President George W. Bush announced a new, U.S. government public-policy initiative: the President's Malaria Initiative (PMI). The goal of the PMI was (and is) to combat the high incidence of malaria in Sub-Sahara Africa. Bush said that the United States had an "unprecedented opportunity” to assist Africa in its development goals, but that "overcoming extreme poverty [in Africa] requires partnership, not paternalism.” He then added

The whole world will benefit from prosperity and stability on the African continent. And the peoples of Africa deserve the peace and freedom and opportunity that are the natural rights of all mankind. We seek progress in Africa and throughout the developing world because our interests are directly at stake.....Next week at the G8 [Summit in Scotland], I will urge developed countries and private foundations to join in a broad, aggressive campaign to cut the mortality rate for malaria across Africa in half. And our nation is prepared to lead. Next year, we will take comprehensive action in three countries-Tanzania, Uganda and Angola - to provide indoor spraying, long-lasting insecticide-treated nets, and effective new combination drugs to treat malaria.... America will bring this anti-malaria effort to at least four more highly endemic African countries in 2007, and at least to five more in 2008. In the next five years, with the approval of Congress, we'll spend more than $\$ 1.2$ billion on this campaign. (Bush PMI Speech) 
This speech and Bush's subsequent speech at the G8 summit in Scotland laid the foundation for the five-year (2006-2010), \$1.2 billion malaria-treatment-and-control initiative now known internationally as the PMI. The PMI became operational in 2006 in Angola, Tanzania, and Uganda. By 2008, it had become operational in a total of 15 African nations, including Liberia. In 2011, the PMI became operational in Nigeria and the Democratic Republic of Congo and the Asian nations comprising "the Greater Mekong Subregion”: Cambodia, China (Yunnan and Guangxi Zhuang), Lao, Myanmar, Thailand, and Vietnam. The 2011 additions were made possible under the Lantos-Hyde Act of 2008, which extended the PMI funding from fiscal years 2006-2010 to fiscal years 2009-2013.

The PMI is implemented by USAID and the CDC and is overseen by a U.S. Global Malaria Coordinator, who is assisted by an Interagency Steering Group, which consists of representatives from the U.S. Departments of State and Defense, the National Security Council, and the Office of Management and Budget (PMI.gov, "PMI Structure”). The USAID-CDC Interagency Working Group spearheads the design and implementation of PMI activities, including program planning, monitoring, and evaluation; staff recruitment and training; technical support, and procurement of antimalarial drugs, bed nets, insecticides, equipment for indoor residual spraying, and related materials. These antimalarial drugs and related malaria-treatment-and-control commodities are usually distributed by the local NMCPs, with help from communityand faith-based organizations and from local and international NGOs. The USAIDCDC Interagency Working Group not only prepares the yearly MOPs (with inputs from administrators of local NMCPs and other health professionals), but also defines PMI activities for each PMI-supported country and sets the eligibility guidelines and selection criteria for PMI support in individual countries.

The eligibility criteria for PMI support mandate that a country must have: (1) a high burden or incidence of malaria; (2) a high potential impact for malaria mortality; (3) a U.S. government "in-country presence" (such as a U.S. Embassy or Interest Section); (4) the political will and leadership at the national level to control malaria and enter into cooperative partnership with the U.S. government; (5) national policies, 
programs, and practices for malaria treatment and control that are consistent with WHO-recommenced malaria-treatment methods; and (6) a grant from the Global Fund for in-country malaria control and in-country involvement by other donor agencies and organizations, such Roll Back Malaria, the World Bank Malaria Booster Program, UNICEF, or UNDP (PMI Strategic Plan 2005). Hence, with Liberia’s selection as a PMI-supported country in 2008, PMI-Liberia automatically became a cooperative partnership between the U. S. and Liberian governments for malaria treatment, control, and prevention. Under this partnership, the U.S. government provides financial and technical support to Liberia through USAID and CDC to fight malaria, while the Liberian government identifies and coordinates the treatment of malaria within targeted populations through the NMCP and the Ministry of Health and Social Welfare (MOH\&SW).

The primary goal of PMI is to reduce malaria-related deaths by 50 percent in participating countries after the first three years of implementation, particularly deaths among children under five years and pregnant women. To achieve this goal, PMI predicates its operations and malaria-treatment-and-control activities in each country on the efficient use of ACT, IPTp, ITNs, and IRS (USAID-Fast Facts). However, because of the unique characteristics of individual nations (i.e., geographic size, population, infrastructural and technological development, language and culture, and the local epidemiology and transmission rates of malaria), one of the main strategies for PMI implementation is an integrated approach to malaria treatment and control that "takes into account the local setting in which the disease occurs” (PMI Strategic Plan 2005). Hence, the 2008 Liberian MOP, a 63-page policy document, details the specific state of malaria and corresponding malaria-treatment-and-control needs in Liberia and maps out strategies for meeting those needs through such activities as training and evaluation, operational work plans, and monitoring of annual implementation goals and outcomes. 
During its first year of operation in 2008, PMI-Liberia established several specific goals intended to help reduce malaria deaths in Liberia by 50 percent and to promote nationwide coverage of the use of ACT, IPTp, ITN, and IRS by up to 85-90 percent by 2010. In collaboration with Global Fund and other partners, during the first year of operation, PMI-Liberia committed itself to the following goals:

1. Procure 1.1 million rapid diagnostic tests (RDTs) for quick and non-laboratory diagnosis of malaria, and provide funding for supervision and monitoring of the use or RDTs.

2. Procure 480,000 insecticide-treated bed nets for free distribution through antenatal clinics; purchase 900,000 treatments of ACTs and related drugs for severe malaria; and strengthen the supply chain and logistics systems for malaria drugs in order to ensure reliable access and a steady supply of these essential antimalarial medications.

3. Work toward strengthening the health information system, including the printing and dissemination of registers, and establishing insecticide-resistance monitoring and sentinel sites.

4. Work with local and international NGOs to support community-based information, education, and communication/behavior change communication (IEC/BCC) campaigns to increase demand for and correct usage of bed nets.

5. Work with other partners to strengthen the capacity of the NMCP and $\mathrm{MOH} \& \mathrm{SW}$ at the central, provincial, and district levels to plan, conduct, supervise, monitor, and evaluate ACTs, ITNs, and related activities.

6. Support pre-service and in-service training and supervisions in malaria-inpregnancy (MIP) to increase demand for IPTp, and support the development of a policy and a national reference laboratory to increase diagnostic capacity and quality. (“2008 MOP” 15-22) 


\subsection{Analyzing the Artifact}

In pentadic criticism, two basic assumptions predominate: (1) language use constitutes action rather than motion, and (2) humans communicate messages as if they were participating in a play. These assumptions are best explained to mean that every human communication involves an action of choice, purpose, and motion, wherein action refers to "the ability of an organism to acquire language or a symbol system" (Foss 355). These assumptions also prioritize the use of rhetoric to project a particular message or point of view to a targeted audience or public. The messages projected can then be analyzed for their rhetorical effects through the use of the elements of Burke's dramatic pentad (act, agent, agency, scene, and purpose) and the corresponding ratios (the frequency and/or relationship of each pentadic element to the other).

When PMI-Liberia came into being in 2008, Liberia was still recovering from the residual effects of a devastating 14-year (1989-2003), civil war. Up to 80 percent of basic healthcare services were still provided by international NGOs and other aid agencies, and the use of ACT, IPTp, ITN, and IRS across the country was still in the embryonic stages. IRS was first used in Liberia during the 1958-61 malaria-eradication campaign by UNICEF and WHO, and ACT was first used in 2003 as part of humanitarian assistance by the international NGO Médecins Sans Frontières (or Doctors Without Borders). However, ITN and IPTp were practically nonexistent in Liberia until after the civil war (“2008 MOP”). This explains why in 2005, only 3.2 percent of children under five with malaria fever had received treatment for malaria within 24 hours, and only 4 percent of pregnant women had received any kind of anti-malaria treatment during pregnancy (LMIS 2005). During the same period, only 2.6 percent of children under five had slept under a bed net, and only 18 percent of households had owned a bed net. IRS use was also limited to displacement camps (LMIS 2005). Hence, the launch of PMI-Liberia in 2008 was a welcome relief in a country where malaria is the leading cause of infant mortality, accounting for 21,000 deaths of children under five each year (“2008 MOP” 5). 
Applying pentadic criticism to the PMI-Liberia artifact and its three basic categories of services provides insight into and understanding of how the local people relate to PMI-Liberia's messages, services, and activities. Pentadic criticism is a method of rhetorical criticism that can be used to analyze a variety of research data. Using Burke’s dramatic pentad, I charted each category of PMI-Liberia services to determine the prevalence of each of the five pentadic elements and corresponding dominant ratios.

The five elements of the dramatic pentad (act, agent, agency, scene, and purpose) constitute the core measures of analysis for any artifact of study in pentadic criticism. In this case, the PMI-Liberia artifact and its three categories (preventive services, case management services, and technical services) can be defined and analyzed only in terms of act, agent, agency, scene, and purpose, and how each of these elements relate to—or act in concert with—each other in generating understanding about the structural features and programmatic goals of the PMI-Liberia artifact. But as one must often reckon in the study of rhetoric or argumentation, it is the rhetorical situation that usually stands out in any piece of writing, analysis, or argumentation, in that it defines the background, context, or circumstances of the particular undertaking. This is why in pentadic analysis the rhetorical situation is often defined by the scene because the "scene is the ground, location, or situation in which the rhetor says the act takes place," which scene encompasses descriptions of the "physical conditions, social and cultural influences, or historical causes” of the artifact under study (Foss 358). As Lloyd Bitzer suggests, the "Rhetorical situation may be defined as a complex of persons, events, objects, and relations presenting an actual or potential exigence which can be completely or partially removed if discourse... can so constrain human decision or action as to bring about the significant modification of the exigence” (6).

For a typical pentadic analysis of the PMI-Liberia artifact, the five elements of the pentad would be defined as:

- act (allocation of $\$ 12.5$ million by the U.S. government)

- $\quad$ agent (implementers of PMI-Liberia)

- agency (purchase of antimalarial drugs and insecticides) 
- scene (the nation and people of Liberia)

- purpose (reduction in malaria-related deaths).

The next step would be to find the dominant element and construct a ratio (e.g., act-agent or act-scene) and begin the analysis and write-up. But Burke insists that the underlining motive for all symbolic human actions is persuasion and that "an act of persuasion is [often] affected by the character of the scene in which it takes place and of the audience to whom it is addressed" (A Rhetoric of Motives 62). This means that for the PMI-Liberia artifact, an interdependent or causal relationship exists between the scene (i.e., the 3.8 million population) and the act (i.e., the $\$ 12.5$ million to combat malaria). It is important to stress the significance of scene-act and scene-agent in Burkean dramatism, given that motivation for a particular human action is often associated with these elements acting together.

In terms of motivation, PMI-Liberia was launched in 2008 at a time when Liberia had no national reference laboratory and no equipment for mosquito collection and identification. There were also no quality-control tests conducted on insecticide use; no pre-IRS environmental assessment, no insecticide-resistance monitoring system; no monitoring-and-coordination plan for malaria-related activities; and no written, national-policy guidelines for malaria diagnosis. Management systems for malaria control and prevention were either dysfunctional or non-existent, and drug vendors were not regulated, so they could sell expired or outdated malaria drugs at will ("2008 MOP” 11). Thus, with $\$ 12.5$ million appropriated for use during its first year of operation in Liberia, PMI-Liberia set out to purchase anti-malaria drugs and related insecticides, using 36 percent of the budgeted amount to procure and distribute ITNs; 33 percent to support improved case management, including the purchase of ACTs and drugs for severe malaria; 10 percent to support program monitoring and evaluation; 2 percent to support malaria treatment for pregnancy women; over 4 percent to support IRS activities; and so forth (“2008 MOP”). All of these activities were undertaken to fulfill PMI-Liberia's original mandate to reduce malaria-related deaths in Liberia by 50 percent in 2010, although this goal was scaled down to 25 percent in 2009 (see Malaria 
Strategic Plan for 2009-15) for a variety of reasons, including the country’s limited technical, professional, and case-management capacities.

Yet the notion that PMI-Liberia could cut malaria-related deaths in half in only three years of operation in the midst of multiple socioeconomic and technical and administrative problems was both an act of remarkable ambition and a significant understatement of the importance of scene in Burkean dramatism. Burke argues that "there is implicit in the quality of a scene the quality of the action that is to take place within it.... [or simply] that the act will [always] be consistent with the scene” (6-7). This means that while purchasing antimalarial drugs and insecticides (agency) and reducing malaria-related deaths (purpose) are important considerations for the overall success of PMI-Liberia, both agency and purpose cannot function without the presence of act (allocation of \$12.5 million by the U.S. government), agent (the organization or PMI-Liberia), and scene (the nation and people of Liberia). Hence, Burke insists that "at the very center of motivational assumptions" lies the ratios of scene-act and scene-agent (A Grammar of Motives 11), which signifies the enduring power of scene in a variety of rhetorical situations and interactions. Burke also insists that human motives are best understood within the context of scene because "motives are shorthand terms for situations” (29).

The scene is the dominate term, and scene-act is the dominant ratio for the PMILiberia artifact. The scene provides the background, location, and circumstances of the hospitals, clinics, and health centers in the cities, local town centers, and villages in the countryside where bed nets, indoor residual spraying, and related antimalarial drugs are distributed and used; it describes a range of people (e.g., mothers, fathers, sons, daughters, children, and elders) yearning to rid themselves of malaria; it represents members of the population who can neither speak nor read and write in English but who are expected to use English instructions to guide themselves in the use of bed nets and indoor residual spraying, and in taking the prescribed doses of ACTs and IPTps in the national fight against malaria. This scenario describes the basic "scene" in the PMILiberia artifact, and the scene tells it all. There is an element of magnanimity associated with the act of the U.S. government providing $\$ 12.5$ million dollars to fight malaria in a 
country in which the general population stands at risk of contracting malaria. This act resonates well with the scene of people, places, and health facilities confronting a high incidence of malaria and malaria-related deaths with limited resources, supplies, and trained personnel. But there is more to this act and this scene regarding the level of involvement of the local people and their language, culture, and traditions in the design and implementation of such lifesaving endeavors.

Burke aligns various philosophical schools of thought with each of the five pentadic elements to account for the depth of meanings and interpretations that each element signifies and to highlight the complexities associated with each artifact of study. He aligns act with realism; agent with idealism; agency with pragmatism; scene with materialism; and purpose with mysticism. These philosophies have wider implications for the study of pentadic criticism, but the point of emphasis here is that blending any two of these pentadic elements together (e.g., scene-act or materialismrealism) underscores the level of complexities that are likely to obtain during analysis of an artifact. Edwin Black also acknowledges the complexities associated with analyzing a rhetorical artifact when he asserts that "the function of [rhetorical] criticism in shaping the ways in which an object will be apprehended brings the style of critical writing to transcend mere embellishment, and to acquire probative force” (xiii). Hence, to appreciate the probative force of the PMI-Liberia artifact on malaria-treatment-andcontrol efforts in Liberia, it was necessary to break down the artifact into three constituent parts or categories and to analyze each category to bring about a full understanding of the role, impact, and challenges of PMI-Liberia in the fight against malaria. 


\subsection{Viewing Categories of PMI-Liberia through the Pentad}

In analyzing the preventative-services category, therefore, we can identify these pentadic elements:

- $\quad$ act (purchase and ship anti-malaria drugs and insecticides to combat malaria in Liberia)

- $\quad$ agent (PMI-Liberia through NMCP)

- agency (distribute and treat targeted population)

- $\quad$ scene (pregnant women, children under five years, and other at-risk groups)

- $\quad$ purpose: to help reduce malaria-related deaths

According to the 2008 MOP, “Although malaria is endemic in Liberia and all persons are at risk, the NMCP strategic plan (2004-2008) regarding ITNs focuses on populations most vulnerable for malaria morbidity and mortality, children under five and pregnant women” (17). This passage demonstrates the effect of scene in that it sends out a clarion call for action against malaria in Liberia, especially among children under five and pregnant women. Hence, the dominate term for the preventive-services category is scene, and the dominate ratio is scene-act. But the strength of this category also lies in how well the targeted population is mobilized to be receptive and enthusiastic about using bed nets and related antimalarial drugs as malaria treatments of choice at the individual level. The Ministry of Health and Social Welfare's endorsement of “Artesunate-amodiaquine (AS + AQ) — [or ACT] as first line treatment of uncomplicated malaria”—alongside IPTp, ITNs, and IRS (“2008 MOP” 10) may also prove inadequate, given the history of the use of ACT, IPTp, ITN, and IRS in Liberia. Hence, it may take some serious adjustments before both the general and vulnerable populations can begin to use these antimalarial drugs and insecticides as first-line malaria treatments for themselves, their families, households, and villages. However, the purchase and shipment of antimalarial drugs and insecticides (act) provides a great 
opportunity for establishing connections with the vulnerable populations of women, children, and other at-risk persons (scene). Without the availability and use of these antimalarial drugs and insecticides, the prospect for getting rid of malaria among these vulnerable populations could prove problematic, especially for people who do not use traditional herbal-treatment substitutes. This is why the efficient distribution of antimalarial drugs, bed nets, and insecticides (agency) to the targeted populations (scene) is bound to have tremendous impact on the quantity and quality of drugs, care, and treatment received by members of these vulnerable populations.

On the other hand, the NMCP is the government's policy body responsible for identifying at-risk sites and population groups across the country and coordinating the distribution of bed nets, antimalarial drugs and insecticides for PMI and other MTCPs operating in Liberia. However, the NMCP is saddled with multiple administrative and technical problems for which it has been unable to perform to full capacity. For example, in 2008, the "salaries and related program costs of the NMCP were covered" through grants from the Global Fund, and the NMCP did not and still "does not have adequate space and office capacity to properly perform their duties in a timely and efficient manner” (“2008 MOP” 32). The NMCP is still rebuilding its operational capacity and storage and distribution channels in order to be able to execute its mandate to the maximum, and this is why the case-management category focuses on improving the management and administrative capacities of NMCP and other public-health facilities across the country.

The pentadic elements of the case-management-services category can be described as:

- act (building professional capacity of health workers and health centers)

- agent (PMI-Liberia)

- agency (empowerment training and communication strategies)

- scene (urban and rural health clinics and malaria-control facilities)

- purpose (promote efficient management control and service delivery) 
At many public-health facilities across Liberia, the "Staff with professional management skills are in severe shortage, including within the NMCP.... [and] local health authorities have been left to fend for themselves, seeking help from available partners including NGOs and FBOs” (“2008 MOP” 11). This shortage of skilled staff presents a serious problem for community-based information, education, and communication/behavior change communication (IEC/BCC) campaigns and related communication strategies intended to increase demand for and correct usage of bed nets, antimalarial drugs, and indoor residual spraying. The 2008 MOP catalogues multiple problems associated with successful implementation of IEC/BCC and related case-management activities in Liberia:

The NMCP has an IEC component in its 2004 strategy document. It is uncertain how completely the ITN IEC strategy has been implemented or how effective it has been. While traveling through Monrovia, several billboards were visible promoting ITN importance and usage. Several posters promoting similar messages were seen in the few health clinics visited. The health facility staff interviewed replied that they encouraged pregnant women and mothers of children under five to acquire ITNs, but unfortunately, they did not know where to obtain them. The staff was unaware of any ITNs available in any shops and did not have any available to distribute. (18)

The extent to which IEC/BCC and case-management activities have incorporated the local culture and traditions_or galvanized active public support and cooperation regarding the efficacy of using bed nets, antimalarial drugs, and insecticides to treat malaria—is highly debatable. Given that the majority of Liberians neither speak nor read and write in English (see chapter 2), the use of English-language billboards and posters in attempts to educate the public about the use of bed nets and related antimalarial drugs raises serious question about the effectiveness of these IEC/BCC activities, as per this statement from the passage quoted above, "It is uncertain how completely the ITN IEC strategy has been implemented or how effective it has been." 
The case-management-services category is characterized by personnel training and supervision at the health-facility level, pre-service training for health-care providers, and communication training executed through IEC/BCC campaigns intended to promote health information about the use of ACT, IPTp, ITN, and IRS among the local population. It also emphasizes strengthening the supply chain and logistics systems for antimalarial drugs. Because this category seeks to empower personnel in both the NMCP and public health facilities across the country through training and capacity building, it has agency as common term and agency-act as dominant ratio. Slack and Wise argue that contrary to the "dictionary definition and popular usage [that] reduces agency to a thing," agency is rather "a process and a relationship” that is not "a possession of agents” or requires "human intention” (117). In other words, developing empowerment and communication strategies (agency) to building professional capacity of health workers and health centers (act) or promoting efficient management control and service delivery (purpose) are not a possession of the PMI-Liberia (agent), but rather a shared relationship that seeks to reduce malaria-related deaths in Liberia through the use of bed nets, antimalarial drugs, and insecticides. The ratio of agency-act also serves to enrich the collaborative efforts needed to achieve the primary goal of the PMI-Liberia artifact through the process of the available means and instruments necessary to achieve such a goal.

For the technical-services category, the pentadic elements can be described as follows:

- $\quad$ act (create laboratory and evaluation systems for health workers)

- $\quad$ agent (PMI-Liberia)

- $\quad$ agency (data collection, procurement, and training)

- $\quad$ scene (health facilities and health-care providers)

- $\quad$ purpose (improve microscopic diagnoses and institute proper healthrecord keeping) 
In 2008, at the main, national, referral hospital,- - the John F. Kennedy Medical Center (JFK) - there was only " 1 microscope in the central lab, 1 microscope in the maternity center, and 2 in the new laboratory building being funded by the Chinese" (“2008 MOP” 28). The 2008 MOP also reports that "Most non-hospital based laboratory facilities operate with only laboratory aides," and that "There is some discrepancy as to the number of laboratory technicians working in Liberia with MOH\&SW putting the number somewhere between 77 and 149, although only 48 were said to be licensed by the National Lab Association of Liberia” (27).

The lack of laboratory equipment and related capacity-building and casemanagement issues still confronted the healthcare-delivery system in early 2011 during my field research in Liberia. Rapid diagnostic tests (RDTs) for quick and nonlaboratory diagnosis of malaria have become standard means of diagnosing malaria across the country, and "Most of the trained technicians in Liberia... are either working in other fields because of better economic opportunity, or working for international NGOs, many centrally in Monrovia” (“2008 MOP” 27). Besides the unreliability of electricity in Liberia (many offices and households use gas generators), the number of laboratory equipment at various health facilities is limited and "most laboratories do not have microscopes whereas some of those with microscopes have them reserved for the work of the program that gave it out, such as TB” (“2008 MOP” 28). These are shortages for which the need for building a national capacity for microscopic-diagnosis confirmation of malaria for all age groups, as opposed to presumptive diagnosis by nurses, doctors, and other health-care workers, underpins the technical-services category.

Within this category, two, long-term-expatriate technical advisors from USAID and CDC direct administrative and technical activities in areas as diverse as monitoring insecticide-resistance for IRS, conducting entomological surveys, and collecting and analyzing routine health-data. The advisors are also involved with planning and implementing malaria vector studies and controls, establishing a central, healthmanagement-information system (HMIS) to collect key data at all levels of health services, establishing an insectary and entomology laboratory for effective malaria 
treatment and control, and monitoring and evaluating program outcomes and impacts. As a result of these activities, the key term for this category is agency. Because the means and instruments (agency) by which data collection, procurement, and training take place are germane to the creation of laboratory and evaluation systems (act), the dominant ratio is agency-act. Agency-scene and agency-purpose are also important considerations for building an efficient technical capacity for NMCP and public-health facilities under the PMI-Liberia artifact, but none of these can be achieved without data collection, procurement, training, and development. Slack and Wise suggest in answer to the question, "What is the benefit of thinking of technology in terms of agency?" that "When we think of ourselves as moving through everyday life, we tend to focus on our encounters with other people and how these encounters alter the character of our day, or even our actions and behavior” (123). Under the technical-services category, PMILiberia is using technology to build various systems for microscopic diagnosis of malaria, and efficient collection of malaria and related health data by creating processes and relationships aimed at reducing malaria-related deaths in Liberia through the effective usage of bed nets, antimalarial drugs, and insecticides.

\subsection{Findings and Conclusions}

François Grin suggests that one of the "single most important concepts in policy evaluations” is that of counterfactual (88). He explains that the term counterfactual refers not "to anything that would be contrary to fact" but rather to "what would occur in the absence of a policy, or ...the relevant alternative” (88). In my analysis of the 2008 MOP, I found several cases of counterfactual, wherein national policies for regulating various aspects (e.g., case-management) of the healthcare-delivery system in Liberia were either non-existent or not enforced. I found that up until 2008, no written national-policy guidelines existed for malaria diagnosis and that failures to enforce provisions of the 2001 National Drug Policy created a situation where "private dealers

freely import, distribute and sell medicines,” including fake or expired drugs (“2008 MOP” 11). This lack of national policies or enforcement mechanisms created new socioeconomic conditions, which demonstrated that national-policy decisions regarding 
the use of ACTs and related antimalarial drugs or malaria-treatment options were not always made in line with the healthcare needs and socioeconomic, political, and cultural realities of Liberia.

I found, for example, that in May 2003 the Liberian government adopted ACTs (notably a combination of Amodiaquine [AQ] and Artesunate [AS] or AS+AQ) to replace Chloroquine (CQ) as first-line drugs for treating malaria at health facilities across Liberia. I found that while the effectiveness of chloroquine against certain strains of p. falciparum and other malaria-producing parasites was still a subject of much public debate in global-health circles in 2003, the government's decision to adopt ACTs was not necessarily based on the results of local empirical studies about the ineffectiveness of chloroquine or the country's healthcare needs. Rather, the government adopted ACTs based on "recommendations" from the WHO and "pressure" from the Global Fund or GFATM (“2008 MOP” 21). The 2008 MOP doesn’t explain the nature of the recommendations from WHO, but it suggests that the government adopted ACTs due to "additional pressure... from the GFATM, which rejected the country's Round 2 malaria grant proposal in 2003” (“2008 MOP” 21). The government subsequently included ACTs as first-line treatment in its Round 3 GFATM malariagrant proposal, which was accepted. However, "The therapeutic efficacy of AQ alone, or the AS+AQ combination, was not studied in the Liberian population prior to its selection as first-line treatment” (“2008 MOP” 21).

Like ACTs, the government also adopted in 2003 the use of intramuscular artemether (AM) and artesunate suppositories as pre-referral drugs for severe malaria without any prior studies among the local population. The government never implemented a planned "pilot evaluation" intended to test the effectiveness of these drugs as a possible treatment alternative for severe malaria (“2008 MOP” 21). From these acknowledgements in the 2008 MOP, a strong case for Grin's notion of counterfactual can be made, especially in regard to the huge vacuums created from the lack of clear national policies to regulate various aspects of healthcare services in the country. A case of counterfactual can also be made for existing national policies that 
either were restructured due to external economic pressure or were not enforced at all due to lack of logistics, trained manpower, and related administrative factors.

My analysis of the 2008 MOP also reveals a common theme, which centers on the question, "How do written and oral communication practices affect negotiation and construction of knowledge in risky situations?” (Sauer 4). Beverly Sauer first raised this question when she examined how uncertainties of the material environment affected communication within large regulatory industries and agencies. She insisted that the ability of rhetors and risk communicators to "address the knowledge, understanding, values, belief systems, fears, hope, and shame of the audiences they seek to persuade” (3) was crucial to communicating with people of diverse cultures and persuasions in risky situations. It is difficult to disagree with Sauer's argument that effective communication rests on a clear knowledge and understanding of the language, culture, values, belief systems, and material environment of the targeted audience. For Sauer's question undergirds the notion that huge reductions in malaria-related deaths were possible during the first three years of PMI operations in Liberia through the use of insecticide-treated bed nets (ITNs), antimalarial drugs (ACTs and IPTps), and indoor residual spraying with insecticides (IRS) alone.

Designers and implementers of PMI-Liberia might have taken for granted the people's belief systems or simply miscalculated the gravity of the residual effects of the 14-year civil war, which resulted in the kinds of socioeconomic and related technical, case-management, administrative, and public-information-dissemination problems discussed throughout this chapter. Evidently, these multiple problems made achieving the goal of reducing malaria-related deaths by 50 percent in three years largely impossible, although not entirely (see Table 4.1).

I also found that while the Global Fund, the WHO, and other international aid agencies (see “2008 MOP” 20-25) may have exerted economic pressure on the Liberian government to adopt ACTs and other new antimalarial drugs as first-line treatments for malaria, the government may have also adopted these drugs as a consequence of relying on case studies (see 2001-03 study in Colombia by Neal Alexander et al. and 2000-07 study in Africa by Abdisalan Noor et al of bed-net use.), and related written accounts 


\begin{tabular}{|l|l|l|l|l|}
\hline \multicolumn{4}{|c|}{ Table 4.1 RBM/PMI Estimates, Progress, and Target Gaps } \\
\multicolumn{1}{|c|}{$\begin{array}{c}\text { Core } \\
\text { Indicators }\end{array}$} & $\begin{array}{c}\text { MIS } \\
\text { Baseline } \\
\text { Goal for } \\
2005\end{array}$ & $\begin{array}{c}\text { RBM/P } \\
\text { MI } \\
\text { Target } \\
\text { Goal } \\
\text { for } \\
2010\end{array}$ & $\begin{array}{c}\text { MIS } \\
\text { Achieveme } \\
\text { nt } \\
\text { Metric for } \\
2009\end{array}$ & $\begin{array}{c}\text { Overall } \\
\text { Target } \\
\text { Gap }\end{array}$ \\
\hline $\begin{array}{l}\text { Estimates of } \\
\text { households with at least } \\
\text { one ITN }\end{array}$ & $18 \%$ & $85 \%$ & $47 \%$ & $33 \%$ \\
\hline $\begin{array}{l}\text { Estimates of children } \\
\text { under five who slept } \\
\text { under an ITN }\end{array}$ & $2.6 \%$ & $80 \%$ & $26 \%$ & $53 \%$ \\
\hline $\begin{array}{l}\text { Estimates of pregnant } \\
\text { women who slept under } \\
\text { an ITN the previous } \\
\text { night }\end{array}$ & n.a. & $80 \%$ & $33 \%$ & $47 \%$ \\
\hline $\begin{array}{l}\text { Estimates of pregnant } \\
\text { women who slept under } \\
\text { ANY net the previous } \\
\text { night }\end{array}$ & $31 \%$ & n.a. & $34 \%$ & n.a. \\
\hline $\begin{array}{l}\text { Estimates of women } \\
\text { who received two or } \\
\text { more IPTP during } \\
\text { pregnancy within a } \\
\text { two-year period }\end{array}$ & $4.5 \%$ & $80 \%$ & $45 \%$ & $35 \%$ \\
\hline $\begin{array}{l}\text { Estimates of children } \\
\text { under five who } \\
\text { received prompt } \\
\text { treatment for malaria } \\
\text { within 24 hours of } \\
\text { developing fever }\end{array}$ & 5.26 & $80 \%$ & $17 \%$ & \\
\hline
\end{tabular}

from other countries about the success rates of these insecticide-treated bed nets and antimalarial drugs. However, whether the government relied on external economic pressure or case studies for its action, vital statistical information from local studies on the populations' perceptions and/or allergic reactions (or the lack thereof) to ACTs and the other new antimalarial drugs may have gotten lost in the process of adopting these drugs. Sauer's contention is underscored by the absence of local studies on the effectiveness of ACTs and other antimalarial drugs prior to their adoption as first-line 
treatments. This development also explains the poor quality of the data available for morbidity- and consumption-based forecasts for the PMI-Liberia due, in part, to weak surveillance and reporting systems (“2008 MOP ”22).

On the other hand, the 2008 MOP contains no evidence of the incorporation of local Liberian culture and traditions in the design and implementation of PMI-Liberia. I found no mentions of words such as "local language," “culture," and "traditions” in the 63 pages of the $2008 \mathrm{MOP}$, although language, culture, and traditions are integral parts of linguistic culture (see chapter 2), and they play an important role in adapting healthrisk communication to the specific cultural contexts of diverse populations. As Rex Veeder suggests, "language is the mediator” by which we can move and act in the world (100), so much so that the "transmission and codification of language" has profound bearings on a "culture's notions of the value of literacy and sanctity of texts" (Schmidt 121). From these accounts by Veeder and Schmidt, it is apparent that language and culture - and by extension cultural traditions - have indelible impacts on the way in which information and communication are structured and disseminated in society. Unfortunately, the 2008 MOP fails to include any communication strategies for educating local populations in their local language and culture about the use of ACTs, bed nets, and related antimalarial drugs and insecticides. IEC/BCC strategies and activities involving the active participation of the local chiefs, community leaders, and other local users of these antimalarial drugs and insecticides were grossly underdeveloped or nonexistent in the $2008 \mathrm{MOP}$.

Of the four MOPs (2008, 2009, 2010, and 2011) for PMI-Liberia, only the 2011 MOP mentions IEC/BCC activities that sought the active participation of local chiefs and other traditional leaders, although the main goal was to effect "behavior change for correct and consistent use of ITNs, [and] acceptance of IRS,” ACTs, and IPTps among the local population, including traditional leaders (“2011MOP” 19). PMI-Liberia's support for the nationwide IEC/BCC strategy of the NMCP "to provide messages through various media including television and radio, as well as through more traditional structures such as tribal chiefs, village leaders and community health 
volunteers" in the 2011 MOP (19) also signals an important development beyond the 2008 MOP. This means that while the participation of traditional leaders in IEC/BCC activities cannot substitute for incorporating local culture and traditions in program design and implementation, it is still an important first step in communicating across cultural boundaries. It signals recognition of the cultural, linguistic, and socioeconomic differences that impel "many patients with fever or other illnesses to consult with a neighbor, traditional healer, or private pharmacy" due to distance, road condition, and shortages in healthcare facilities (“2011 MOP” 24).

I found a few references to "traditional midwives" in the 2008 MOP, but the main emphasis was on how to train these midwives to assist with Malaria-in-Pregnancy (MIP) efforts, not on how to incorporate the local cultures and traditions of midwives into program design and implementation. In spite of this emphasis, the 2008 MOP did not contain any IEC/BCC strategies and activities for educating the public about MIP, or any of the related antimalarial drugs and insecticides such as, ACT, IPTp, ITN, and IRS. Hence, the 2008 MOP provided no opportunity for incorporating local culture and traditions in program design and implementation. It instead provided an opportunity for evaluating the healthcare needs of Liberia and for mapping out appropriate strategies for malaria control and prevention that involved the active participation of every segment of the population, including traditional leaders.

In Section 4.3, I underscore the complexities and prospects of a rhetorical artifact, such as PMI-Liberia, in the fight against malaria in Liberian society. Edwin Black argues that criticism of any rhetorical artifact demands full disclosure because criticism has "no relationship with its subject other than to account for how that subject works” (18). Hence, in analyzing the 2008 MOP, which embodies the operational plans, strategies, and goals of PMI-Liberia, I sought to understand not only how the artifact works, but also how it incorporates local culture and traditions in program design and implementation. To this end, Pierce suggests that an artifact is nothing more than a "tangible, retrievable trace or recording of the original messages" (47) originating from specific geographic or material environments. Blending together Black's notion of criticism and Pierce's notion of a rhetorical artifact, I sought to understand the PMI- 
Liberia artifact through the lenses of Burke’s pentad (act, agent, agency, scene, and purpose). Because this artifact encapsulates areas as diverse as personnel training and development; technical support; case management; and procurement of antimalarial drugs, bed nets, laboratory equipment, and insecticides, it had scene-act as dominant ratio.

By using pentadic criticism to analyze the 2008 MOP, I sought to identify with Burke in the study of motives with respect to how the PMI-Liberia artifact might facilitate communicating health information and related risk messages to diverse cultural populations by incorporating local culture and traditions in program design and implementation. The elements of Burke’s pentad rely on differing worldviews or philosophical schools of thought to provide more dramatic and broader insight into how understanding local cultures and traditions can avert potential problems with the healthcare-delivery services in society, including services delivered by MTCPs in Liberia.

Pentadic criticism of the 2008 MOP is also helpful in understanding the contributions of PMI-Liberia to malaria-treatment-and-control efforts in Liberia from an outsider's perspective, which can provide baseline indicators for future studies of MTCPs and the role of local culture and traditions in general malaria-abatement programs.

This analysis contributes to rhetorical theory through its emphasis on the process of communicating health-risk messages across diverse cultures and populations and the ethnographic implications for incorporating local culture and traditions in program design and implementation. As Carolyn Miller suggests, the writer or communicator must endeavor at all times to understand the conditions of his or her own participation in a communicative community in terms of "the concepts, values, traditions, and style which permit identification with that community” (617). Like Miller, Kelli Cook argues that in today's world, technical communicators "need to be multiliterate, possessing a variety of literacies that encompass the multiple ways people use language in producing information, solving problems, and critiquing practice” (5). Both Miller and Cook recognize that language and culture are interwoven attributes of human communication, 
and that together they play a pivotal role in communicating across cultures and in fostering human understanding through multiliteracies.

Pierce's concept of “inherited worldview” and Searle’s concept of deep background and local background can be easily meshed with Burke's circumference of the scene to probe further as to whether or not the goals enunciated and the successes achieved by PMI-Liberia’s 2008 MOP can be extended beyond its current target locations, both within and outside Liberia. The original goal underpinning the launch of PMI in 2005—not PMI-Liberia in particular—can be easily linked to Burke’s notion for expanding the circumference of the scene, but they are still lessons to be learned from the implementation of PMI-Liberia artifact that could affect the design and implementation of malaria-treatment-and-control activities in Liberia, Africa, and other parts of the world.

Marris, Langford, and O’Riordan argue that even as sociologists probe human social processes, they readily acknowledge that "individuals and groups often "reframe their interpretations of the context of a hazard stress according to a serious of communication procedures, in which the media are also involved” (636). The point of emphasis here is that as long as there exits individual and cultural differences, effective communication can only take place through a collaborative space that looks at the cultures and traditions or "local background" (Searle) of all the parties involved. According to Marris, Langford, and O’Riordan, while Mary Douglas and other proponents of Cultural Theory celebrate cultural biases inherent in four unique worldviews (e.g., fatalists and egalitarians) as a basis for understanding individual risk perceptions and risk factors in society, they still lack general agreement on the stability or mobility of these worldviews. And this disagreement is borne of the cardinal argument as to whether or not "individuals will choose to attach themselves to institutions with the same type of social organization in different spheres of their lives... and will therefore adhere consistently the same cultural bias whatever the social context?” The answer to this question is both simple and complex, depending on whether or not one wagers on stability or mobility. But an even more fundamental question lies in whether or not we can find anyone in our common world who can defy 
human evolution and stick entirely to one action or another? This is exactly the question that undergirds the PMI-Liberia artifact and the Liberian government and people.

The challenges for incorporating local culture and traditions in the design and implementation of MTCPs and related health-risk messages in Liberia are as important as local variables such as health facilities, equipment, trained manpower, and available communication and transportation systems. Yet the need for action is overwhelming, and it will take the collective efforts of all involved to ensure success. This is where the circumference of the scene can be extended and expanded to include both the international and local remedies and processes that work best rather than place all bets on a one-size fits all proposition. Clearly, Burke might have felt that the circumference of the scene could be extended as the need arises in order to obtain the best results and not only the available results. 


\section{Chapter 5 Research Findings}

\subsection{Introduction}

In this chapter, I discuss the results of my field research in Liberia. From February to May 2011, I traveled back-and-forth between Monrovia (the national capital) and Buchanan (a coastal city 88 miles east of Monrovia) to interact with designers, implementers, and beneficiaries of malaria-treatment-and-control programs (MTCPs). Representatives of these groups from the National Malaria Control Program (NMCP) and the Ministry of Health and Social Welfare (MOH\&SW), and from the national offices of the World Health Organization (WHO), the Global Fund for Aids, Tuberculosis, and Malaria (Global Fund), the United Nations Children Fund (UNICEF),

\section{Ethnicity of Interview Participants}

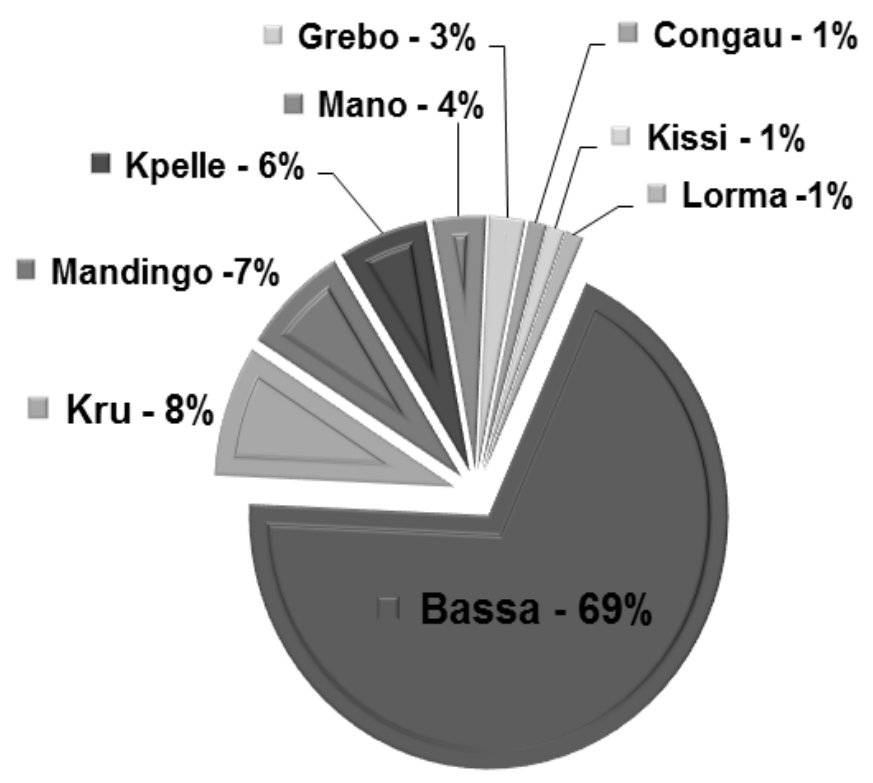

Figure 5.1: Ethnicity of Interview Participants 
the United Nations Development Program (UNDP), and the U.S. President’s Malaria Initiative (PMI), among others, completed survey questionnaires about their various operations. Beneficiaries of MTCPs — such as traditional leaders and elders, heads of households, mothers, and young adults-were interviewed in their homes, offices, and towns in and around Buchanan about their knowledge of malaria and their use of bed nets and other services provided by MTCPs, such as indoor residual spraying and Artemisinin-based combination therapy (ACTs). Hence, the two sources of primary data analyzed for the findings in this chapter are survey-questionnaire data and fieldinterview data.

The purpose of this analysis is to determine the extent to which-if anyMTCPs in Liberia incorporate or fail to incorporate local culture and traditions in program design and implementation. Because unique societal and cultural factors—such as language, linguistic culture (see Chapter 2), politics, health regulations, and belief systems-are important considerations for communicating health and related risk messages to diverse populations, both the survey questionnaires and the interviews included basic demographic questions about the participants, such as age group, gender, community of residence, length of residence, agency affiliation, length of service, and job function. The survey questionnaires and interviews also included questions about the participants' knowledge of program design, implementation, and outcomes and about the participants' methods and practices for communicating health risks in the language, culture, and traditions of the local people.

For the survey questionnaires, a total of 21 people responded, one more than the original goal. The overall rate of return for the survey was 21 out of the 50 questionnaires emailed or hand-delivered to potential participants.

For the interviews, a total of 84 people responded, four more than the original goal. The participation rate for the interviews either fell below or exceeded the targeted number in each participant category (e.g., young adults or heads of household). For example, of the 20 traditional leaders/elders targeted for the interviews, only 11 participants identified themselves as either an elder or a traditional leader. And although 


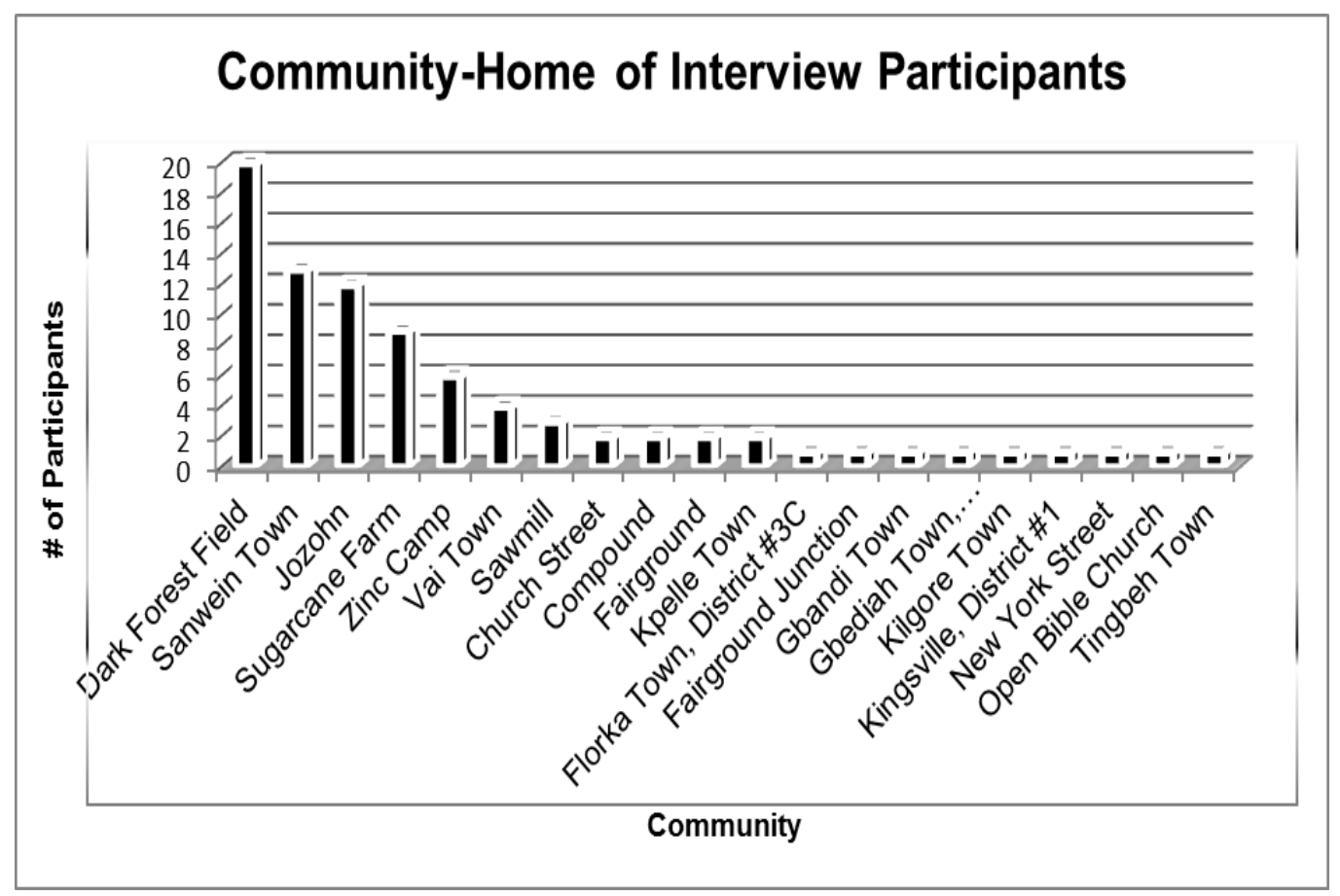

Figure 5.2: Interview Participants by Community

only 20 heads of household were targeted, 35 participants identified themselves as the head of a household.

The rest of this chapter discusses the demographic data of participants and the results of the data analysis for each research question.

\subsection{Demographic Data}

Of the 84 interview participants, 49 (58.33 percent) were male and 35 (41.67 percent) were female. The oldest participant was 74, and the youngest participant was 18. Eleven participants ( 7 males and 4 females) were 60 and over, including 8 in their 60s and 3 in their 70s; 11 (8 males and 3 females) were 50-59; 40 (18 males and 22 females) were 30-49; and 21 (12 males and 9 females) were 18-29. Similarly, 21 participants (14 males and 7 females) identified themselves as young adults, while 35 participants (28 males and 7 females) identified themselves as heads of a household. Eleven participants (8 males and 3 females) identified themselves as traditional leaders/elders, while 20 participants identified themselves as mothers. The numbers in 
these categories sometimes overlap because, for example, a person identified as a traditional leader based on official title may also be the head of a household or a mother. This was the case when three of the participants identified themselves as church leaders/pastors and heads of a household, while several other participants-ranging in age from 18 to 60 - also identified themselves as either mothers and heads of households or as young adults, mothers, and heads of households. Seventy-eight (92.85 percent) of the interview participants identified themselves as Christian, and 6 (7.15 percent) identified themselves as Muslim.

Although no interview participants claimed to be traditional spiritualists and/or oracle worshippers, some of the participants from a fishing town near Buchanan spoke openly of how they and other townspeople made annual offerings to a local oracle or deity to increase their fishing bounty and to preserve the peace and safety of the town. Sixty-seven of the 84 interview participants (79.76 percent) spoke Bassa, the predominant local language, while 56 participants (66.66 percent) also spoke English (either “Liberian Standard English” or Liberian Pidgin English). Many interview participants were either bilingual or multilingual. Thirty-one participants (36.90 percent) spoke Bassa and English; 6 (7.14 percent) spoke Mandingo and English; 5 (5.95 percent) spoke Kpelle and English; 4 (4.76 percent) spoke Kru and English; 2 (2.38 percent) spoke Lorma and English; 2 (2.38 percent) spoke Grebo and English; 1 (1.19 percent) spoke Kissi and English, and 1 (1.19 percent) spoke Bassa and Kru. One participant spoke five languages (Bassa, Kpelle, Mano, Gio, and English), and another participant spoke four languages (Mano, Bassa, Gio, and English). Twenty-three participants (27.38 percent) spoke only Bassa, while three participants spoke only English, although they acknowledged being born into a local Liberian ethnic group. During the interviews, those participants who spoke only Bassa, and those who spoke Bassa and English but felt uncomfortable responding to the interview questions in English were aided by volunteer Bassa-language interpreters. 
Participants from nine of the 16-plus ethnic groups of Liberia participated in the interviews (see Figure 5.1). A majority of the participants came from one ethnic group: Bassa (58 or 69.05 percent). The interview participants also came from 20 local communities within Buchanan and its environs (see Figure 5.2). The highest numbers of participants came from Dark Forest Field (20 or 23.81 percent); Sanwein Town (13 or 15.48 percent); Jozohn (12 or 14.29 percent); Sugarcane Farm (9 or 10.71 percent), and Zinc Camp (6 or 7.14 percent). On average, the participants have lived 13.01 years in their various communities, with length of stay ranging from one week to 60 years. Because the survey questionnaires focused on demographic data—such as agency affiliation, length of service, and job function — the age group and gender respondents were not easily identifiable. However, of the 21 completed questionnaires, only two were originally delivered to and retrieved from females. It may be, therefore, that 19 males and 2 females participated in the survey. The survey respondents also came mostly from NMCP, Africare Liberia, USAID, Mentor Initiative, UNICEF, WHO, and UNDP. Of the 21 respondents, 12 (57.14 percent) listed their job function as implementers; 6 (28.57 percent) as administers; 2 (9.52 percent) as designers, and 1 (4.77 percent) as a technical adviser. No respondent identified himself or herself as "staff," although this was one of the job-function categories. A majority (11 or 52.38 percent) respondents had spent 3 to 4 years on the job; 6 (or 28.57 percent) spent 0 to 2 years; 3 (14.29 percent) spent 7 or more years; and 1 (4.77 percent) spent 5 to 6 years.

\subsection{Survey-Questionnaire Data Indicators and Implications}

The survey questionnaire consisted of 18 questions that asked about the respondents' demographic characteristics (questions 1-4; already discussed in Section 5.2); their primary medium of health-risk-communication (questions 5-9); and relevant program design and outcome (questions 10-18). The five questions about primary medium of health-risk communication sought to ascertain the language and medium through which the respondents communicated information about MTCPs and health risks to the local population and whether or not elements of the local culture and traditions were included in the process. In response to question 5, which was on 
language use, 7 (33.33 percent) respondents indicated that they use only Liberian Standard English to communicate with the target population, while three said they use only Liberian Pidgin English (see Chapter 2 for differences between Liberian Standard and Pidgin English).

However, 11 (52.38 percent) respondents said they communicate with their target populations through a combination of Pidgin English and a variety of local languages, mainly Bassa, Kpelle, Vai, Kru, Mandingo, Grebo, Mano, Gio, and Lorma. In response to question 6 , which was on medium of communication, the majority respondents (12 or 57.14 percent) said they use mainly radio to communicate, while 5 (23.81percent) said they use mainly public speeches, and 3 (14.28) said they use mainly traditional towncriers. Ten respondents said they use a combination of radio, public speeches, towncriers, newspapers, drama, Internet, posters, flyers, and communityawareness campaigns to communicate with their target audiences.

In response to question 7, "Does the design of risk-communication messages for your MTCPs include elements of the local culture and traditions?” the majority of respondents (18 or 85.71 percent) answered "yes," while 1 (4.76 percent) respondent answered "no," and 2 (9.52 percent) respondents answered neither "yes" nor "no." Question 8 asked respondents to explain how they incorporated or planned to incorporate elements of the local culture and traditions into the design of their riskcommunication messages. Six (28.57 percent) respondents opted not to answer, and 15 (71.43 percent) respondents provided various perspectives on incorporating local culture and traditions into their health-risk-message design. Many respondents saw cultural incorporation as the use of local languages, songs, drama scripts, drumming, and radio or television spot-messages. Others felt that the active involvement of local chiefs as participants, interpreters, or facilitators in malaria-treatment-and-control sensitization workshops sufficiently signaled the incorporation of local culture and traditions in program design and implementation.

To one respondent, "The use of National Traditional Council of Liberia and Crusaders for Peace [a local theater group] is a sure way of incorporating the cultural elements” in health-risk-message design. Another respondent thought the use of 
Liberian Standard English was necessary for the "adaptation of teaching (written) materials into Liberian context.” A third respondent spoke of the complexity of cultural adaptation and noted that within the Edina community of Grand Bassa County, some of the local people "use a particular leaf that has a very offensive odor to drive away mosquitos and [they] prefer it to mosquito nets that are believed to be used for dead bodies.”

One elderly participant identified the leaf in question as pou-yong-pou, which he said is often placed on a room door or hung up in the room to drive away mosquitos. Several other participants said they often burned palm kernel in the home to drive away mosquitos. Hence, from information gleaned from interview participants, it is apparent that the use of various herbs to treat malaria or to drive away mosquitos to prevent malaria is not an isolated practice in Edina but a widespread practice throughout Grand Bassa County in particular, and among the Bassa people of Liberia in general. However, because traditional association of nets (including bed nets) with dead bodies was not probed during the study, only anecdotal accounts of this association among the Bassa are available. In Bassa culture, whenever someone is seriously ill, a common practice is to quarantine that person under a canopy of white linens - similar in structure to bed nets - while the traditional zoe, spiritualist, or herbalist treats that person. And if that person should die, he or she is kept under the canopy in isolation until the appropriate burial rituals. Given this use of a canopy for the seriously ill and the dead among Bassa people, it is possible that some Bassa people associate white bed nets with the white canopy of linens for dead bodies. The use of bed nets of colors other than white could present an opportunity to test this belief system.

Question 9 asked respondents what they might do differently in the future to include local culture and traditions in the design of their risk-communication messages. Fifteen respondents offered a wide-range of suggestions, while 6 respondents did not answer this question. For those who responded, common strategies centered on increasing the number of local vernacular languages in disseminating health-risk messages; involving local community leaders and town chiefs in the development of health-risk messages and related materials; and going beyond the pre-testing stages of 
every message to obtain various interpretations and understandings of the target communities. One respondent suggested the inclusion of "traditional healers in the conversation [about malaria], especially those who claim to have a cure for all diseases, particularly malaria, [in order to] find out what they are using as 'cure' [and] educate them.” Another respondent suggested that "the people’s cultural values and the benefits they would get from what you are asking them to do" should be key considerations in designing health-risk messages.

The last eight questions on the survey asked respondents to name the types of services their MTCP provided to the public, to rate their program design and outcome, to rate the interactions between their MTCP and beneficiaries, and to indicate if their MTCPs was designed inside or outside Liberia. Five respondents said their MTCP provided public-health-education-and-training services; 5 provided bed net-distribution services; 1 provided microscopic-laboratory-competency training; 1 provided technical advice in the areas of finance and logistics; 7 provided multiple services (i.e., bed net distribution, bed net-use training, indoor-residential spraying, ACT distribution, and public-health-education training), and 1 provided other services. Five respondents rated the services provided by their MTCP as excellent, 12 as good, and 4 as great. Five respondents also rated the interactions between their MTCPs and recipients of the services they provided as excellent, 14 as good, and 2 as great.

Question 13 asked, "What do you believe have been the outcomes of your program in reducing malaria in Liberia?” Thirteen respondents rated their programs as moderately successful, and 8 rated their programs as greatly successful. In answer to question 14, 15 respondents indicated that the outcomes of their programs fulfilled the original goals; 4 said the outcomes exceeded the original goals, and 4 said the outcomes failed to fulfill the original goals. However, when asked in question 15 to explain the indicators on which responses to questions 13 and 14 were based, one respondent explained: "In question 13, I answered and said 'moderately successful' because some areas are not reachable due to bad road networks. And question 14, our answer was ‘doesn't fulfill goals' due to shortage in materials or low quality materials.” Three respondents didn't answer question 15, but the 18 who did explained why they felt their 
MTCPs were either moderately successfully, greatly successful, or unsuccessful in terms of program design, implementation, and outcomes. One respondent rated his or her MTCP as greatly successful because “The recent malaria indicator survey (2009) shows a reduction in mortality and morbidity in malaria cases and deaths due to malaria....This is the main objective of the NMCP.” Another respondent felt his or her MTCP did not fulfill its stated goal because "Under-five [children under five years of age] utilization of nets remains relatively low, though the ownership is high; under five mortality (due to malaria) not significantly reduced; no stock out of antimalarial medicines at health facilities.”

Although question 15 asked respondents to provide supporting documents, (such as annual reports) to explain why they rated their MTCPs as either successful or unsuccessful, only two respondents from the NMCP provided the relevant documentation. Many respondents indicated either that they had no documentation to provide or that they wanted a separate request for documentation beyond the survey questionnaire. As one respondent wrote, “[ $\mathrm{I}$ am] Not authorized to attach project document [to the survey questionnaire] without formal request in advance.”

\subsubsection{Respondents' Perceptions of Culture in Program Design}

In answer to question 16, "Was the MTCP you work for designed inside Liberia?” a majority respondents (15 or 71.43 percent) answered “yes;” two (9.52 percent) answered “no,” and four (19.05 percent) answered “don’t know.” The last two questions on the survey asked respondents to explain how they felt about incorporating local culture and traditions in the design and implementation of MTCPs in Liberia 
Table 5.1 Respondents Discuss Cultural Inclusion in Message Design

In future, the inclusion of local cultural values and beliefs will greatly assist in the preventing of malaria. Also our organization in the future will be total involvement of the locals; traditional town crier in local language.

Increase the number of messages in the local vernacular and directly involve the local leaders and town chiefs in the disseminating of the information to the community.

Perhaps include traditional healers in conversation, especially those who claim to have a cure for all diseases particularly malaria, find out what they are using as "cure," educate them.

Well, in designing messages you have to consider the people's cultural values and the benefits they would get from what you are asking them to do. So yes we considered all of those during message design.

The use of local culture and tradition in the design and implementation of MTCPs in Liberia is essential to fully disseminate the MTCPs' messages to a broad spectrum of the Liberian population since many are illiterate, emerging from various cultural backgrounds.

Our messaging includes traditional songs and drumming and names. This is intended to show ownership. We are also working with the traditional council of Liberia doing advocacy

Local culture and traditions is necessary in the design of malaria intervention because Liberians are traditional people and most times traditions affect project implementation especially with regards to achieving outcomes.

I have travelled frequently in the hinterland with MTCP messages. A village whose English speaking is not common, we involve our relationship with our traditional people by using the culture for instance the Poro so that our messages can go across to the target population.

The use of National Traditional Council of Liberia and Crusaders for Peace is a sure way of incorporating the cultural elements. Moreover, all produced materials are pre-tested twice; developmental and end user testing. These exercises [are] also keen about cultural factors.

Some people in Liberia (Edina community in Grand Bassa) use a particular leaf that has a very offensive odor to drive away mosquitos and prefer it to mosquito nets that is believe to be used for dead bodies. Therefore, the message is that is most effective for malarial prevention. 
(question 17), and to make any comments about MTCPs in Liberia not already covered in the questionnaire (question 18). The answers to these two questions were insightful and intriguing in that, in many instances, they underscored the complexity of communicating across cultures (see Table 5.1), wherein linguistic and cultural differences and local socioeconomic circumstances can tip the balance in how one accepts or reacts to a health-risk message. For instance, one respondent felt his or her MTCP has "already incorporated local culture and traditions" in program design and implementation by working with the National Traditional Council of Liberia ${ }^{4}$ on various advocacy campaigns for malaria treatment and control. However, other respondents felt there was a pressing need for MTCPs to incorporate local culture and traditions in program design and implementation; for example, one respondent stressed the need "to create a more direct link which will improve local ownership and increase compliance.”

For question 18, 11 (52.38 percent) respondents had no comment, while 10 (47.62 percent) respondents made comments about the structure and communityoutreach activities of MTCPs. One respondent suggested that MTCP activities should be extended to "all sectors of life and places—villages, towns, and cities in Liberia." Another responded said the Ministry of Education "should incorporate MTCP in the national curriculum to be taught in school as separate subject.” A third respondent suggested that "Cases treated for malaria should be confirmed before treatment to ensure that true malaria cases are diagnosed and treated in order to show the impact of the various interventions on malaria incidence control.” A fourth respondent suggested the destruction of mosquito breeding sites through the use of outdoor spraying to help reduce both the mosquito population and the burden of malaria. A fifth respondent called for MTCPs to decentralize their current community-sensitization campaigns and advocacy meetings at the county-level to include "smaller sub-political divisions such as districts and major towns.” This respondent also suggested that MTCPs be revamped

\footnotetext{
${ }^{4}$ The National Traditional Council of Liberia is a representative body of traditional chiefs from Liberia's 15 political subdivisions. The Council is headed by Chairman, who serves as chief spokesperson and advocate for traditional value in Liberia. The Council has existed for decades as mostly a ceremonial body until recently the Council became visible and vocal in national affairs under current chairman, Chief Zanzar Karwor of Grand Bassa County.
} 
to involve more local NGOs and community-based organizations (CBOs) in the program implementation.

Some respondents argued that because local NGOs and CBOs are strategically situated in Liberian society, they are in a position to better understand the culture and traditions of Liberia and to help members of local communities to understand the issues of malaria treatment and control more clearly and, thus, take actions on their own to eliminate malaria-breeding sites in their locales. Hence, respondents felt that local NGOs and CBOs should be involved in the design of communication strategies for the community-sensitization campaigns of MTCPs and other such strategies geared toward reducing the effects of malaria in local communities.

\subsection{Interview Data Indicators and Implications}

The interview data was generated from 37 structured interview questions that sought to understand participants’ demographic backgrounds (questions 1-8 discussed in Section 5.1); participants' knowledge and perception of malaria and of bed-net use (questions 9-25); and participants’ health-risk-communication practices within their respective ethnic groups and cultures (questions 26-37).

In answer to question 9, "have you ever heard of malaria?” all 84 interview participants answered yes, and all but two participants had suffered from malaria. However, many of the participants tended to identify more easily with the local, traditional name for malaria than with the word malaria. Because many of the interview participants either spoke Bassa or belonged to the Bassa ethnic group, the Bassa word sun-nee or sun-glie dominated answers to question 10, "What is the name for malaria in your local language?” Other popular local names for malaria mentioned during the interviews included gbeley-yan (Kpelle); garatumon (Kissi); clan-glen (Kru); neebruen or ouou (Grebo); farlee, gelegbanee, or sumaya (Mandingo), and nennie (Mano).

The two participants who claimed to have never suffered from malaria had no logical explanation for their unique situation in a tropical country ravaged by the disease, but scientists have suggested that the sickle cell trait, which is common in West Africa, provides limited immunity to the disease. In 2005, a study of 1,000 people (3 
months to 84 years) in the Kenyan rift valley found that the sickle cell trait "gives an increasing amount of protection against malaria," especially in the first 10 years of a child's life (NewsRx.com). In a 1955-56 study of sickle cell trait in Liberia lasting 10 months from September 1955 to August 1956, Frank Livingstone found that the sickle cell trait is prevalent among almost all the ethnic groups of Liberia, including the Bassa (33). However, I could not confirm as to whether or not some of the interview participants had sickle cell trait or ever suffered from sickle cell anemia, since I had no way of knowing outside clinical testing.

In answer to question 12, "If no, what would you do if you got malaria?" one of the two participants who did not have the disease said he would go to the hospital to be properly diagnosed and treated; the other said he wouldn't know what to do if he got malaria. For those 82 participants who had suffered from malaria, 28 (34.15 percent) said they took only Western medicine (e.g., seek hospital treatment); 16 (19.51 percent) said they took only traditional medicine (e.g., use herbs or go to traditional healer), and 38 (46.34 percent) said they took a combination of Western and traditional medicine. The most popular Western malaria treatments the participants mentioned were amodiaquine, chloroquine, oral quinine, and Fansidar. The most popular traditional medicines were ganagana, sekou toure leaf, jologbo, and plum tree bark.

Many of the participants described ganagana, sekou toure leaf, jologbo, and plum tree bark as consumable but bitter liquids intended for the relief of malaria for several days or weeks without any dosage limitations or side effects. However, several of the participants reported adverse reactions to amodiaquine and chloroquine. One participant lamented his adverse reactions to both chloroquine and amodiaquine:

They call the tablet amodiaquine, but when you take it, just like you're going to die. It is not an easy tablet. People at first used to take 4, 5, 6, or 7 tablets per day. This time, we reduced it to 2-2, and it is not easy. When you take that malaria drug just like you're going to die. It is strong. Well, that is the only one that can use [work on] me. Sometimes I take chloroquine, but if I use the chloroquine too my ears and everything 
can be, yes.... Well it takes long time before I can hear correctly again. (Interview participant)

Another participant said he opted to take only "country” (traditional) medicine because “the drug amodiaquine when I take it my whole ear can lock. So I don't take it. I usually take jologbo. It is bitter; it takes the malaria out of you.” These participants' reactions to amodiaquine underscore and confirm concerns about adapting ACT (Artesunate-amodiaquine combination therapy) as first-line treatments for malaria without any prior empirical studies in the Liberian population on the therapeutic efficacy of ACT (see chapter 4), especially amodiaquine, which has such side effects as nausea, vomiting, itching, stomach upset, headache, loss of vision, skin rash, muscle pain, hearing loss, and graying of the hair (Medindia.net).

\subsubsection{Participants' Knowledge and Perception of Bed Nets}

Because distribution and use of insecticide-treated bed nets are integral parts of the operational strategy of MTCPs in Liberia (see chapter 4), all interview participants were asked question 14: “Do you use bed nets to fight off malaria?” Twelve (14.29 percent) participants answered “no,” 69 (82.14 percent) answered “yes,” and three (3.57 percent) answered that they had bed nets at home but didn't use them (see Table 5.2 for representative sample of participants' knowledge and perception regarding the use of bed nets and insecticides). The majority of those participants who answered "no" said they usually used mosquito chord (which burns like incense) or spray to deter mosquitos. One participant simply said he used “country medicine,” while another said he usually burned palm kernels in his home to deter mosquitos. One participant said she didn’t use bed nets because "I just don't like it.” The three participants who had bed nets but didn't use them said they avoided the nets because of the heat. A majority of the participants (39 or 46.43 percent) first learned about bed nets via radio; 20 (23.81 percent) through health clinics; 13 (15.47 percent) through word of mouth, and 12 (14.29 percent) through a combination of sources, including NGOs and communityhealth workers. 
In response to question 21, “How long have you used bed nets?” 25 (29.76 percent) participants said they didn't know, and 59 (70.24 percent) said they have used bed nets for an average of 4.44 years, (within a range of 1 to 21 years). Responses to question 23, "How many people in your household use bed nets?" ranged from 1 person to 20 persons. As per the 2008 Malaria Operational Plan (MOP) for Liberia (discussed in chapter 4), the national policy undergirding bed-net distribution by MTCPs is three bed nets per household; this is obviously not adequate for households that may include 20 members or more. One participant confirmed this problem when he lamented, "If you have four or five rooms, they only give three” bed nets regardless of family or household size. Another participant lamented the size of the bed nets: "I have a family bed, and they give me a single-bed mosquito net, and it can’t cover my bed. And mosquitos will go all through there, and it is just a matter of waste of time."

The words "mosquito nets" and "bed nets" are used interchangeably, even though one respondent to the survey suggested that "Mosquito net...[should be] used instead of bed net to remove the perception that [nets should only be used on beds rather than on] all sleeping places (mats, mattresses, on the floor, etc.)." Whatever issues interview participants had regarding the name, size, or quantity of bed nets distributed, more than 90 percent of them relied exclusively on free bed nets distributed by MTCPs and NGOs.

In questions 24 and 25, participants were asked to state how people in their ethnic groups felt about using bed nets to combat malaria and how they themselves felt about using bed nets. Question 37 asked participants to recommend any changes in the way that information about bed nets was being communicated. For question 24, 47 (55.95 percent) respondents rated the bed nets as "alright," ”fine,” or "good," while 37 (44.05 percent) rated the bed nets as being "too heatly" and full of "chemicals" that irritate the skin. One participant said people in his ethnic group harbor "negative perception” about bed nets retaining too much heat in the dry season, so they use the 


\begin{tabular}{|c|c|c|}
\hline \multicolumn{3}{|c|}{ Table 5.2 Participants' Perception of Bed Nets and Insecticides Use } \\
\hline $\begin{array}{c}\text { Supportive } \\
\text { of Bed-Net Use }\end{array}$ & $\begin{array}{l}\text { Not Supportive } \\
\text { of Bed-Net Use }\end{array}$ & $\begin{array}{l}\text { Preference for } \\
\text { Bed-Net or } \\
\text { Insecticide Use } \\
\end{array}$ \\
\hline $\begin{array}{l}\text { I have not actually taken } \\
\text { a survey, but I think } \\
\text { people are enthusiastic } \\
\text { about using the net }\end{array}$ & $\begin{array}{l}\text { Bed net is very weak and } \\
\text { the chemical burns the } \\
\text { skin. The chemical } \\
\text { should be cut down; } \\
\text { maybe it is too strong so } \\
\text { it's burning the skin }\end{array}$ & $\begin{array}{l}\text { I don't have } \\
\text { interest in the } \\
\text { spray because the } \\
\text { spray brought } \\
\text { more mosquitos. I } \\
\text { trust the bed net } \\
\text { more than the } \\
\text { spray }\end{array}$ \\
\hline $\begin{array}{l}\text { I think I feel fine because } \\
\text { if Kwee people [Kwee is } \\
\text { Bassa word for white } \\
\text { people or educated } \\
\text { people] bring something } \\
\text { you are not supposed to } \\
\text { dispute it. }\end{array}$ & $\begin{array}{l}\text { Well, actually, I don't } \\
\text { really feel fine [using the } \\
\text { net] because it is not } \\
\text { comfortable for } \\
\text { me....People say you use } \\
\text { the net to protect the } \\
\text { mosquito. But } \\
\text { sometimes I strongly feel } \\
\text { like it doesn't. Because } \\
\text { they give you; I have a } \\
\text { family bed and they give } \\
\text { me a single-bed mosquito } \\
\text { net and it can't cover my } \\
\text { bed. And mosquito will } \\
\text { go all through there, and } \\
\text { it is just a matter of waste } \\
\text { of time. And the heat } \\
\text { again, so I don't feel } \\
\text { comfortable using that. }\end{array}$ & $\begin{array}{l}\text { Bed net is good but } \\
\text { spray really kills } \\
\text { the mosquito } \\
\text { because when we } \\
\text { get out to use the } \\
\text { outside bathroom, } \\
\text { the mosquito } \\
\text { enters [the room } \\
\text { again] }\end{array}$ \\
\hline $\begin{array}{l}\text { I feel very fine [using } \\
\text { bed nets] because I can } \\
\text { sleep sound if I sleep } \\
\text { under it }\end{array}$ & $\begin{array}{l}\text { I feel reluctant [using bed } \\
\text { nets] because my country } \\
\text { medicine can help me }\end{array}$ & $\begin{array}{l}\text { Use bed nets and } \\
\text { the spray. The } \\
\text { spray beings in } \\
\text { mosquito more }\end{array}$ \\
\hline $\begin{array}{l}\text { Oh, we are happy [using } \\
\text { bed net], but mosquito is } \\
\text { not really in this area, } \\
\text { except when storm } \\
\text { comes }\end{array}$ & $\begin{array}{l}\text { Feeling bad because } \\
\text { malaria in me, I go to } \\
\text { bed, and I don’t sleep. I } \\
\text { have malaria but I don't } \\
\text { use bed net }\end{array}$ & $\begin{array}{l}\text { Too much water in } \\
\text { the spray. They } \\
\text { shouldn't put more } \\
\text { water }\end{array}$ \\
\hline $\begin{array}{l}\text { I am happy [using bed } \\
\text { net] but the one I have is } \\
\text { now rotten }\end{array}$ & $\begin{array}{l}\text { Don't like it [bed net] } \\
\text { because it burns my skin }\end{array}$ & $\begin{array}{l}\text { Yes, the spray is } \\
\text { better than the bed } \\
\text { nets }\end{array}$ \\
\hline
\end{tabular}


bed nets only during the rainy season. For this participant, using bed nets during the rainy season felt fine, but he still felt bad because "under summer days, I can be embarrassed by the dry season. Temperature hot.” Regarding question 25, 78 (92.86 percent) respondents said they felt happy or fine about using bed nets, while 6 (7.14 percent) respondents expressed reservation about the heat and chemicals from the bed nets. One participant said, "I don’t like it because it burns my skin.” Another said, "I feel fine, but I can't use it because of the heat. But I like to use it because I don't like mosquito while lying under the net.” In answer to question 37, a majority of participants

either didn't know what to recommend, or said they were satisfied with the current way information about bed nets was being communicated. One respondent to the survey said, "Education and cultural understandings must be promoted on all fronts if bed nets are to be used, in [door] or outdoor residual spraying accepted and appreciated, and public health messages understood and embraced.”

\subsubsection{Participants' Perception of Language and Culture}

Question 26 asked, "What things do you consider health risks in your tribe or culture?” Three (3.57 percent) participants answered “don’t know.” Eighty-one (96.43 percent) participants said they considered health risks in their tribe to be anything that affects human health or proves dangerous to humans, including the following: open pits, dumpsites, latrines, diarrhea, tuberculosis, malaria, pneumonia, mosquitos, cow-flies, roaches, and dirty drainages and ponds. One participant said, “Ah, actually, what we really consider health risk is the issue of dumpsite, dirty water, and they [the people] have been taught to keep all those things away from themselves.” On how they treated health risks in their tribe or culture, one participant said, “I don’t know about culture business, ooh!” The rest of the participants suggested possible remedies, such as cleaning their environment, cleaning the dumpsites, burning or burying garbage, advising children not to toilet around the house or to bury their feces, and creating public education and awareness on how to keep the surrounding area clean.

Because many of the participants had no access to electricity, pipe-borne water, inside bathrooms and flush toilets, and public dumpsites or garbage-collection systems, 
it was not uncommon to find open toilets, open dumpsites, and outside bathrooms within their communities. One participant said his community held meetings twice a month for the purpose of cleaning their community and their homes.

In communicating health risks in their tribe or culture, many of the participants said traditional modes of communication-such as the use of towncriers for door-todoor messaging, drumming, community elders and block leaders, and community meetings - are still being employed, in addition to radio announcements, community outreach, and word-of-mouth messages.

When asked "Should your language, culture, or tradition matter in communicating health risks to you?” a majority of the participants (83 or 98.81 percent) answered “yes,” while 1 participant (1.19 percent) said it didn’t matter. One participant said due to sensibilities inherent in each culture or language, it was essential to communicate health-risk messages in the local language. He explained that a word such as condom may be inappropriate in Bassa and other local ethnic groups because "to talk about puberty areas is not good in culture.” Another participant added, "Yes, it [the language] very much matter. The culture varies. In the West, you can talk about genital

\begin{tabular}{|l|l|}
\hline \multicolumn{2}{|c|}{ Table 5.3 Participants' View of Language and Culture } \\
\hline $\begin{array}{l}\text { My language is more important because } \\
\text { it is my tribe and I understand it better. }\end{array}$ & $\begin{array}{l}\text { My language is the best. } \\
\text { Because it is my language I } \\
\text { born in and understand. }\end{array}$ \\
\hline $\begin{array}{l}\text { Because I was born in that language, in } \\
\text { that tradition, I will hear it and know the } \\
\text { detail without asking you. }\end{array}$ & $\begin{array}{l}\text { It is very important; that part } \\
\text { of culture. It is the best way } \\
\text { to have someone } \\
\text { communicated with. }\end{array}$ \\
\hline $\begin{array}{l}\text { My language and culture important } \\
\text { because we are the Bassolian respect } \\
\text { our culture. }\end{array}$ & $\begin{array}{l}\text { Culture is important because } \\
\text { some people can't } \\
\text { understand English and } \\
\text { when I speak Bassa they } \\
\text { understand it better. }\end{array}$ \\
\hline $\begin{array}{l}\text { It is better to use traditional languages } \\
\text { because many of our elderly people } \\
\text { don't understand English. Call the elder } \\
\text { people together and educate them. }\end{array}$ & $\begin{array}{l}\text { It's important to speak in my } \\
\text { dialect; I am a native } \\
\text { woman. }\end{array}$ \\
\hline
\end{tabular}


areas, [but] it is a problem” to talk about genital areas in many of the local cultural groups. Many of these local cultures still reserve roles for women and the elderly, so their language places much emphasis on respectability, decency, and communal lifestyles. According to one participant, “Some people don’t want their names to be exposed” in any health-risk situations, so that they prefer for the health messages to be communicated through the local language to avoid miscommunication.

Many other participants gave varying reasons why they thought health-risk messages should be communicated in the local language (see Table 5.3 for a representative sample of the reasons provided by participants for why their language and culture matter in communicating health risks). One participant contrasted the folk belief of "anything can kill you" with efforts to communicate health-risk messages outside the urban centers: "Outside the city, once the people are farming, it can be very difficult to get them together to spread the message; especially if you talk about mosquito; they will complain that anything can kill you, so da mosquito business I will leave my farm and sit here? When the people are farming, it looks difficult to get them together at times.”(Cultural translation: Anything can kill me; hence, I'm not going to waste time going to meetings about mosquitos or bed nets when I can be farming.)

Participants were asked to explain "how and in what ways?” their language, culture, and traditions mattered in communicating health risks. In response to this question, 14 (16.67 percent) participants said “not sure,” and 70 (83.33 percent) participants provided various narratives. One participant said communicating in her local language was very important "because what you understand is what you have interest in." Another participant addressed this same theme: "It’s important to speak in my local dialect; I am a native woman.” Many participants said communicating in their local languages was important for easy understanding and cultural identity. One participant said health-risk messages should be communicated in Bassa because most of the people in Buchanan and the whole of Grand Bassa County spoke Bassa. Two participants said in spite of their individual levels of proficiency in English, there were 
still times when they wanted to be communicated with in their local languages. One of these participants provided this account of the importance of communicating in his local language:

It [local language] is the greatest thing our people understand. Even I who here where I say I try to learn one or two book, graduated from high school and going to college, there are certain, certain things if you communicate with me, I still want to understand it in my dialect. So it is very very important to our people that you use your own local dialect to them so that they can be able to understand it clearly. I don't care how you speak the lowest English, the Liberian English, to them, there is a need, a need to speak that dialect.

For many participants, communicating directly through their local languages is essential to understanding health-risks and other messages. As one of the participants previously noted, there are certain sensibilities and cultural norms associated with each local language that cannot be accurately conveyed through the use of English or English-language interpreters. These sensibilities have deep roots within each language and culture. One participant said she would rather settle for interpretation from English to Bassa than accept the message entirely in English: "I prefer my language because I understand it better. I always ask for interpreter.” One participant suggested that the two preeminent, traditional learning institutions in Liberia, the Poro (male) and Sande (female), "thrive on the local language," which demonstrates an affinity by the participants for their local languages.

\subsubsection{Summary and Transitions}

The survey-questionnaire data and the field-interview data for this analysis consisted of 18 and 37 questions, respectively, which sought to understand the participants' demographic backgrounds (e.g., age group, agency affiliation, and job function), medium and language of health-risk-communication, and whether or not elements of the local culture and traditions were incorporated into the design and implementation of MTCPs. Both sets of data reveal a common theme in two key areas: 
cultural identity and language proficiency. Each group of principals—such as MTCP administrators, designers, and implementers (survey questionnaires) and MTCP beneficiaries (field interviews) — wrestled constantly to retain ownership or cultural identity of their respective spheres of influence.

The MTCP administers and implementers wanted to change the behaviors of the beneficiaries to fully embrace the services they were offering, and the beneficiaries wanted the administrators and implementers to embrace and incorporate themselves and their cultures in the design and implementation of the MTCPs. In the survey questionnaires, one respondent suggested that "The use of National Traditional Council of Liberia and Crusaders for Peace [a local theater group] is a sure way of incorporating the cultural elements” in health- risk-message design. Yet another respondent spoke of the complexity inherent in cultural adaptation by illustrating how some people in the Edina community in Grand Bassa County associated bed net-use with dead bodies (see Chapter 5, Section 5.3).

On language proficiency, participants in both the survey questionnaires and the interviews relied on multiple languages in communicating across cultures. However, MTCP administrators and implementers had a definite preference for the use of "Standard English,” while MTCP beneficiaries had a definite preference for Bassa and other local languages. One respondent to the survey stressed the need to "Involve adaptation of teaching (written) materials into Liberian context with use of Standard English," while one interview participant spoke highly of the importance of the use of his local language in communicating with him: "Because I was born in that language, in that tradition, I will hear it and know the detail without asking you."

These are clear examples of the complexities of communicating across diverse cultures and populations. Many respondents urged that health-risk messages be conveyed in their native language (or dialect) and stressed that only then would such communication be truly effective. However, there is also evidence that language competence alone may not be sufficient. 
To be truly effective, health-risk communicators must understand not only the language of their audience, but also the audience's larger culture, of which language is a part. For example, as one respondent to the survey noted, "The use of local culture and tradition in the design and implementation of MTCPs in Liberia is essential to fully disseminate the MTCPs' messages to a broad spectrum of the Liberian population since many are illiterate [and] emerging from various cultural backgrounds.” This may also suggest the need to increase the number of local vernacular languages used in disseminating health-risk messages.

Participants' knowledge and perceptions of bed nets and the use of bed nets by MTCPs in the fight against malaria in Liberia were also common themes in both sets of data. During the interviews in Buchanan and its environs, I learned that many people had bed nets in their homes. In addition, the hotel I stayed at in Buchanan and many of the private homes I visited had bed nets. Yet the lack of active involvement of traditional leaders in the design and implementation of MTCPs, especially with respect to bed nets, prompted one local town chief at a malaria-control-sensitization workshop in Tubmanburg, Bomi County to ask that traditional leaders be involved with bed-net distribution.

Given these perspectives, chapter 6 explores and recaps the insights gleaned from the literature review and from the three sets of data unpinning this dissertation. It also advances suggestions and recommendations for adapting health-risk communication to the specific cultural contexts of diverse populations. The fieldinterview data showed that all participants used some form Western and traditional medicine, or a combination of both, to combat malaria. And whether Western-educated or not, almost all the participants thought that using a local language for communicating information about malaria and other health risks could have a significant impact on the overall success of MTCPs. 


\section{Chapter 6: Conclusions and Contributions to the Field}

\subsection{Introduction}

In this final chapter, I provide a summary of the problem statement, recap the insights gleaned from the literature review, and explore and explain the findings and conclusions that emerged during this study. Whether Western-educated or not, almost all of the participants in this study thought that the use of local languages to disseminate health-risk messages could significantly enhance health-risk communication. The fieldinterview data shows that all participants in this study used some form of traditional medicine, Western medicine, or a combination of both to prevent and treat malaria. I conclude this chapter by advancing recommendations for future studies.

\subsection{Summary of the Problem}

The purpose of this study was to assess MTCPs in Liberia in order to find out what effects - if any-local culture and traditions have on the process of communicating health risks to people of diverse cultures. As a teenager in Liberia in the 1970s, I lived near a public landfill that polluted the entire community. Because there was no public-health information or health-risk-communication action plan to help the community cope with the effects of the landfill, many of us in the community frequently came down with malaria, high-fever, rashes, and other illnesses due to the invading armies of mosquitoes, flies, and rodents. We were also subjected to the daily pungent smells of garbage and other wastes dumped into the open landfill. This experience, and similar experiences in my adult life, provided the impetus for a research paper I did for my risk communication class in 2008 on the subject of malaria control in Liberia.

From the literature review for that paper, I found that international NGOs and other aid agencies—-such as the World Health Organization (WHO); the U.S. President's Malaria Initiative (PMI); and the Global Fund for HIV/AIDS, Tuberculosis, and Malaria (Global Fund)—spent hundreds of millions of dollars each year to combat 
malaria in Africa. I found that much of this money is spent on the purchase, transport, storage, and distribution of insecticide-treated bed nets, Artemisinin-based combination therapy (ACT), intermittent preventive treatment for pregnant women (IPTp), and indoor residual spraying (IRS) with insecticides for use by local African communities. Yet malaria still kills an African child every 30 seconds and costs Africa an estimated US\$12 billion each year in lost productivity due to employee absenteeism and related healthcare spending (WHO Fact Sheet 94).

For Liberia, an estimated US\$40 million is spent by international NGOs and other aid agencies each year to combat malaria, which is equivalent to 10.78 percent of the country’s 2009-2010 national budget of US\$371 million. Yet, in spite of this huge sum and the growing widespread use of bed nets, ACT, IPTp, and IRS across the country, malaria continues to be a serious public-health hazard in Liberia, killing about 21,000 children each year and accounting for about 38 percent of out-patient visits and about 44 percent of in-patient deaths (The Analyst). Liberia's 3.8 million people are also at risk of malaria due to the country's vast wetlands and tropical rain forests, which are prime habitats for malaria-bearing mosquitoes. Liberia is home to the four main global strains of malaria parasites, including the deadly Plasmodium falciparum, which affects 66 percent of children under five in Liberia (2009 MIS Update 1). In that 2008 risk communication paper and in this dissertation, my assumption is that given the huge sums of money spent on malaria-treatment-and-control activities in Liberia and other African countries, the main cause of ineffective malaria-treatment-and-control programs may not be the lack of money and medicine-although these are important—but failure to incorporate local culture and traditions in the design and implementation of MTCPs.

I raised four basic questions to test this hypothesis: (1) Why have local and international efforts in malaria treatment and control not succeeded in eradicating malaria in Liberia and other African countries? (2) What effects do local Liberian culture and traditions have on the process of communicating health risks to affected populations? (3) To what extent do the design and implementation of MTCPs impact malaria-treatment-and-control outcomes in Liberia? and (4) How do constraints-such as lack of adequately trained staff, limited funding, cultural resistance, corruption, and 
government bureaucracy —impact health-risk communication in Liberia? To answer these questions, I collected and analyzed three sets of data: (1) existing policy documents on MTCPs in Liberia (such as PMI); (2) survey-questionnaire data; and (3) field-interview data.

\subsection{Purpose and Recap of Literature Review}

The purpose of this study was to assess MTCPs in Liberia in order to find out what effects-if any-local culture and traditions have on the process of communicating health risks to people of diverse cultures. According to Hahn and Inhorn, "Those who interact with foreign cultures have a moral obligation to take those cultures seriously, including their social organization and values” (10). This means that societies once thought of as distant in their socioeconomic, political, and cultural outlooks — and situated thousands of miles away_are today expected to work more closely together in various fields of human endeavor due to the advent of globalization. Jan Nedeverveen Pieterse suggests that "globalization is a long-term historical process" that crisscrosses several ancient and modern civilizations and cultures dating as far back as 500 BCE (24).

This meshing of ancient and modern civilizations and cultures into collaborative partnerships and cooperative economic ventures demands a collective defense against global health risks and transnational diseases, such as malaria, tuberculosis, and HIV/AIDs. This meshing of cultures also explains why health; safety; and environmental-risk messages must be tailored to and disseminated among audiences based on the characteristics of their local cultures and traditions. But culture is a complex phenomenon. It is not simply the way of life of a group of people in a particular setting (e.g., a nation, a community, or a society); it is the food we eat, the clothes we wear, the music we play, and the languages we speak.

Culture gives us a sense of identity and belongingness, especially in our interactions with others. As Raymond Williams says, "culture is ordinary” insofar as we perceive it as a sort of routine activity that empowers us to wake up each morning, go to work or school, eat, play, and sleep at night only to wake up the next morning and start 
all over again. Hence, every human action is influenced by culture in that all human beliefs and social practices are byproducts of the culture to which we belong. Anthropologist Mary Douglas’s grid/group analysis or Cultural Theory (see chapter 1) presents a clear case of how our individual cultures or worldviews can impact how we perceive and react to risks and health-risk messages.

Hahn and Inhorn insist that "local populations, not the outsiders, are the experts on their own sociocultural environment” (9). This is why the active involvement of local populations in the design and implementation of global health programs is essential for program success. As Vinay Kamat says, "Local acceptance of global health programs — which are often designed in 'headquarters' in the West—rests on the ability of public health professionals to tailor, hybridize, or indigenize top-down, one-size-fitsall, interventions to the local level” (15). Kamat insists that to be successful, healthintervention services must take into "account not only the society for which the intervention is intended, but also its social, economic, and political environment” (15).

Kamat's argument is supported by the WHO, which insists that mitigating local and global health risks in the twenty-first century imposes "a shared responsibility" upon nations, peoples, and cultures of the world, in order to ensure "equitable access to essential care for all” (GHR 5). Accordingly, the task of communicating health-risk messages across diverse cultures and populations requires close global collaborations and clear understanding of the local cultures and traditions of individual nations and communities.

\subsection{Methods and Findings}

I used qualitative research methods—such as survey questionnaires and field interviews - to collect the data for this study, and I used rhetorical theory (i.e., rhetorical criticism) to analyze the data. During field research in Liberia, 21 designers, administrators, and implementers of MTCPs completed survey questionnaires about the design, implementation, and outcomes of their MTCPs. Eighty-four beneficiaries of MTCPs — such as traditional leaders and elders, heads of households, mothers, and young adults-answered interview questions about the methods and practices by which 
they communicate health risks in their local languages, cultures, and traditions. For an existing-policy document, I downloaded the PMI’s 2008 Malaria Operational Plan (MOP) for Liberia and analyzed it using Kenneth Burke's dramatic pentad (see chapter 4). I analyzed all three sets of data to determine the extent to which local culture and traditions are incorporated in MTCPs in Liberia. I analyzed this data for possible inaccuracies in program reports about goals and levels of cultural adaptation and for confounding variables, such as dollars invested per person served and appeals to celebrity status. I also examined how the distribution of antimalarial bed nets has been modified (or not) in response to cultural beliefs that associate bed nets with the dead or seriously ill (see the Edina example in chapter 5).

Several findings emerged during my analysis of the data. First, both administrators and beneficiaries of MTCPs showed a tremendous sense of courage, commitment, resilience, and pragmatism to live and work under dire socioeconomic conditions, such as degraded road and communication networks, lack of electricity and pipe-borne water, and poor health facilities with limited doctors, nurses, and medical supplies. Second, local and expatriate MTCP workers frequently traveled outside Monrovia on these degraded roadways to the remotest parts of the country to conduct malaria-sensitization workshops and/or to distribute bed nets and to spray homes with insecticides. Third, I found the local people to be very receptive to hearing and learning new ideas, and many demonstrated these traits by suspending their daily chores to participate in the field interviews in Buchanan, sometimes well into the late evening under candlelight or lantern.

However, the local people's choice of malaria treatment and control was not always influenced by the vigorous national efforts to promote the use of bed nets, ACTs, and indoor residual spraying as first-line treatments for malaria. Of the 84 interview participants, 69 said they had used bed nets to combat malaria, while 12 didn't have bed nets, and 3 had bed nets but didn't use them due to heat and other reasons. All interview participants had heard of malaria and bed nets, but of the 82 participants who frequently suffered from malaria, the majority (46.34 percent) said they used a combination of traditional and Western medicine to treat malaria; 34.15 
percent said they used only Western medicine; and 19.51 percent said they used only traditional medicine (see chapter 5). Only two participants had bought their own bed nets; the majority relied exclusively on donated bed nets.

For many local and expatriate MTCP workers, incorporating local culture and tradition into program design and implementation meant involving local chiefs and other members of the National Traditional Council of Liberia as facilitators and interpreters at malaria-sensitization workshops and creating radio and television spotmessages in selected local languages. However, many beneficiaries of MTCPs didn’t see these activities as cultural incorporation. Many only had vague knowledge about how to set up a bed net on their own; 90 percent said they only used a bed net because it was given to them. Some beneficiaries did not use the nets regularly due to heat and the impregnated chemicals, which itch the skin. Some beneficiaries no longer permitted indoor residual spraying in their homes out of the fear- - founded or not—-that the spraying invited more mosquitos than it eliminated due to excessive use of water in mixing the chemicals (stagnant water is a common breeding ground for mosquitos).

Cultural resistance might have been an underlining motive for those interview participants who used only traditional medicine to treat malaria. One participant wanted the bed nets to be in colors other than white (without stating why), and one survey respondent mentioned that people in the community of Edina associated bed nets with the dead and, hence, would rather use a traditional remedy to drive away mosquitos. The use of English or a local language to communicate health-risk messages was a thorny issue among participants. Administrators of MTCPs tended to prefer the use of English to communicate, while MTCP beneficiaries tended to prefer the use of their local languages. One MTCP administrator suggested that MTCP promotional materials be adapted to "Liberian context with use of Standard English." For one MTCP beneficiary, however, nothing could replace his local language: "Because I was born in that language, in that tradition, I will hear it and know the detail without asking you." These complexities in communicating health risks across cultures and diverse populations demand further research beyond this dissertation. 


\subsection{Effects of Local Culture and Traditions on Communicating Health Risks}

In chapter 1, I discussed Deborah Lupton's belief that people are more likely to measure and identify with risks and risky situations based on their preexisting knowledge, discourses, and other dispositions. To Lupton, no risk is "fully objective or knowable outside of [one's] belief systems and moral position” (29). Lupton's view is supported by Mary Douglas and other proponents of the cultural theory of risk who insist that risk is a socially constructed phenomenon that is as unique to each society as that society's culture. Douglas says that while her grid/group theory originally applied to only Africa, her "renamed and sharpened” cultural theory applies globally. This theory assumes four types of cultural bias, each of which "is based on a type of stable organisation that could not endure if the cultural underpinnings were eroded" (7). Hence, Douglas suggests that adherents of each cultural bias will always "be at war with one another" (7). Her characterization of risk as resulting from competing cultural biases of members of society best explains why local and international efforts at malaria treatment and control have not succeeded in eradicating malaria in Liberia and other African countries. Based on their cultural biases, international NGOs and local governments and populations are often at loggerhead as to which malaria-treatmentand-control medicines work best in each African community.

For example, an analysis of the 2008 MOP (see chapter 4) reveals that even as international NGOs and the national government and people work together to combat malaria in Liberia, there is a perception gap between the two as to which malariatreatment-and-control options are best suited to the country. Upon the insistence of an international aid agency, the Liberian government had to change its national-malariatreatment policy or risk losing millions of dollars in aid. As a result, the first-line malaria treatment was changed from chloroquine to ACT without any prior studies among the local population to test the efficacy and/or side effects of ACT. Thus, many MTCP beneficiaries interviewed for this study took only part of the prescribed drug combination and dosages, due to adverse reactions, including severe headaches, vomiting, weakness, and/or dizziness. All categories of interview participants reported 
these reactions, including traditional leaders, mothers, young adults, and heads of households. Young adults and persons 30-49 were more likely to continue to take the drugs occasionally in spite of adverse reactions, while traditional leaders and persons over 50 were more likely to take traditional medicine, drips, Fansidar, and other overthe-counter drugs than they were to take ACT (especially amodiaquine) again.

Although many interview participants had bed nets in their homes, some did not use the nets at all, and others used the nets only occasionally (mainly during the rainy season). The participants' knowledge and perception of bed-net use varied widely; however, all had heard of bed nets, and more than 80 percent had bed nets at home. Surprisingly, participants who didn't use their bed nets regularly came from all age groups. The common reasons for not using the nets were heat and chemical in the nets. Because a majority of the participants infrequently use the nets, it was difficult to know the efficacy of bed-net use. However, one mother didn't suffer from malaria and so didn't use either a bed net or ACT, but she did use a bed net to protect her child. Several other mothers used bed nets for themselves and their children, and some elderly participants had bed nets that were old and practically useless, with many holes running through them.

\subsubsection{How the Design and Implementation of MTCPs Impact Malaria- Treatment Outcomes}

Of the 69 interview participants who use bed nets to combat malaria, only 5 knew how to set up a bed net themselves. The majority had bed nets set up in their homes by field workers from local and international NGOs and government agencies on the day the nets were first donated to them. This might be because instructions for setting up the bed nets are written in English, and a majority of the MTCP beneficiaries cannot read or write English. Even those who could read and write in English might not have been expected to set up the nets on their own. I reached this conclusion based on these field observations: 1) Some of the participants could neither speak English nor read and write in English; 2) Some of the participants indicated that the nets continued to be permanent fixtures in their homes from the day community-health workers first 
installed them; and 3) some of the participants said that although those who donated the nets taught them how to set up the nets, they were still uncertain as to how to properly set up the nets on their own.

Two malaria-sensitization workshops for local chiefs held in Buchanan, Grand Bassa and Tubmanburg, Bomi County—hosted by the NMCP during the period of my field research—provided some useful clues about bed-net use among MTCP beneficiaries. At both of these workshops, the local chiefs asked for more information about bed nets and indoor residual spraying and what role they might play in malariatreatment-control programs. At Buchanan, one chief wanted to know the difference between bed nets and indoor residual spraying and how bed nets might affect marital relations, given his child shares the room with him and his wife. Another chief wanted to know why strangers were being sent to spray their homes after they had complied with an MNCP request to send residents of their towns to be trained in the use of indoor residual spraying. At Tubmanburg, one local chief asked for traditional leaders to be involved with bed-net distribution, while another participant wanted to know why those promoting the use of bed nets through behavior change were not taking the lead in the use of bed nets. Although limited and anecdotal, these accounts reflect sentiments worth exploring when measuring how the design and implementation of MTCPs impact malaria-treatment outcomes.

Although all 21 survey respondents rated the effects of their MTCP on reducing malaria in Liberia as either moderately successful (61.90 percent) or greatly successful (38.10 percent), they differ greatly on what they might do differently in the future to include local culture and traditions in the design of their risk-communication messages. Five respondents left the question blank. The 16 respondents who answered the question commented on two aspects of MTCPs: (1) message design, and (2) program design and monitoring. For message design, respondents stressed the need to

- Include local chiefs/traditional leaders and their comments and strategies in message and material development in order to promote ownership and acceptance of program messages. 
- Increase the number of messages in the local vernacular and directly involve local leaders and town chiefs in disseminating information to the community.

- Undertake movie productions that will convey in a more practical sense (audio/visual) what is portrayed using local vernaculars and scenes to give the audience a more realistic picture of the message.

- Design health-messages that consider cultural values and the benefits people are likely to get from listening to and acting upon such messages.

- Seek approval for message design in collaboration with the local people, and involve role-play and drama in local dialects.

- Pretest all health-promotional messages with target MTCP beneficiaries, and follow-up beyond pre-testing to obtain various interpretations and understandings of the target communities.

For program design and monitoring, the respondents stressed the need to:

- Track down progress of malaria-control interventions in the general population if prevention and immunization are to be achieved.

- Conduct house-to-house visitations in order to evaluate the need for bed nets, including insecticide-treated nets.

- Establish a monitoring mechanism to measure the effectiveness of the traditional leaders' advocacy, since the results of quarterly dip-stick studies ${ }^{5}$ are not detail as it relates to the chiefs' community activities.

These accounts suggest that more work is needed in order for the design and implementation of MTCPs to impact malaria-treatment outcomes in Liberia in more meaningful ways. As one respondent said, incorporating "Local culture and traditions is necessary in the design of malaria intervention because Liberians are traditional people and most times traditions affect project implementation, especially with regards to achieving outcomes.” In spite of the fact that the majority respondents 15 (71.42 percent) said that their MTCP fulfilled its goals, there is still much room for

\footnotetext{
${ }^{5}$ A dip stick study is a tracking or ad hoc study that seeks to measure progress within a business organization by comparing projects undertaken at various time intervals (e.g., 2008 MOP versus 2009 MOP).
} 
improvement with respect to arriving at an outcome that involves incorporating local culture and traditions in program design and implementation. As one respondent noted, "The incorporation of local culture and tradition in the design and implementation of MTCP in Liberia will help communities better understand the concept of malaria control as their own health benefit and will help them to take ownership of the program.” This self-ownership and community-empowerment may very well be the sort of future role or outcome for MTCPs in the fight against malaria.

\subsubsection{Funding and Other Constraints on Health-Risk Communication}

Effective health-risk communication is often impacted by multiple constraints, such as limited funding, lack of adequately trained medical and professional staff, cultural resistance to the prescribed treatments (e.g., bed nets and insecticide use) , and language proficiency (i.e., clear understanding of the language in which a message is communicated). Among these constraints, however, funding seems the most troubling. Funding for healthcare services is never adequate in any society due to emergent new diseases demanding huge expenditures in medical research and treatment; an ailing elderly population demanding costly medical treatment and care; a growing youth population demanding pediatric, preventive-health, and dental services; and shortages of health facilities and medical practitioners demanding huge costs in infrastructural development and training.

For a country like Liberia, which is still recovering from a 14-year civil war, the funding for healthcare services is never adequate. Of the US\$372-million 2009-10 national budget, only $\$ 18.8$ million was allocated to the central health agency, the Ministry of Health and Social Welfare (MOH\&SW), to provide healthcare services to hospitals, clinics, and schools in the country's 15 political subdivisions. This allocation was shared with the Ministry’s Bureau of Curative Services (\$13.3 million)—which supervises and coordinates healthcare-delivery services in the country at the community and health-facility levels—and with the Bureau of Preventive Services (\$1.7 million), which oversees the activities of national programs tasked with the prevention and control of communicable, infectious, and preventable diseases. Surprisingly, minus 
personnel and other administrative costs, the budgeted amount for drugs and medical consumables was only $\$ 1.2$ million for curative services and only $\$ 10,000$ for preventive services (2009-10 draft budget, Ministry of Finance).

These funding constraints adversely impact not only health-risk communication but also health-policy formulation. The choice of ACT (or Artesunate and Amodiaquine combination) as the first-line treatment for uncomplicated malaria was influenced by the work of an international NGO, Doctors Without Borders or Medecins Sans Frontieres (MSF). MSF first introduced the use of this drug combination in 2003 without any prior statutory approval. Subsequently, the Global Fund demanded a policy change in first-line treatment from chloroquine to ACT as a condition for funding. Because the initial use of ACT was not regulated, and the policy change didn't result from prior drug-efficacy-and-resistance studies among the local population, many health workers are reluctant to prescribe ACT to patients, and many patients are reluctant to use ACT as their first-line treatment for malaria. The authors of the 2010-15 NMSP acknowledge the Liberian "health professionals' reluctance to use amodiaquine” as first-line treatment for malaria, and the "low patient adherence to [the] treatment protocol” for ACT (NMSP 16), but they argue that there is a probable explanation for this outcome: "Due to the humanitarian situation...[in 2003], most of the ACTs brought into Liberia by NGOs were administered based on dose by age instead of [dosage by] weight, thus leading to some reported 'severe' side effects” (NMSP 21). The implication inherent in the 2010-15 NMSP is that the refusal of many health professionals to prescribe ACT to patients is due not to the absence of efficacy-andresistance studies but to the prescription method used by the NGOs that originally prescribed the drugs.

In spite of the change in policy, chloroquine is still widely used across Liberia. The 2009 Liberian Malaria Indicator Survey (LMIS 2009) shows that 28 percent of children under five were treated with chloroquine for uncomplicated malaria, as compared to 30 percent who were treated with ACT (LMIS 2009 xvii). Given this, the 2010-15 NMSP urges that spirited public-awareness campaigns be conducted to change this behavior before the constant use of chloroquine (several of the four major strains of 
malaria virus have developed resistance to chloroquine) prevents "reduction in morbidity and mortality” (21). The NMSP goes on to attribute the near parity in the use of chloroquine and ACT among children under five-and among the adult populationto the limited supply of ACT at private health facilities, where 46 percent of the general population gets its treatment (NMSP 22).

According to the 2009 NMCP Annual Report (NMCP Report), although more than 2.64 million doses of ACT were brought into Liberia in 2009 by various donorsincluding 1.3 million doses by PMI, 1.25 doses by GFATM/UNDP, and 89,000 doses by the Chinese Government - only 805,082 cases were treated with ACT (NMCP Report). In the same year, the PMI brought into Liberia 236,000 tablets of Fansidar (78,666 doses at three tablets per dose) to treat pregnant women and 850,000 Rapid Diagnostic Test Kits (RDTs) for malaria diagnosis (Global Fund/UNDP also contributed 450,000 RDTs in 2009). During 2009, PMI and other donors also brought into Liberia 1.24 million bed nets for distribution to areas with high incidence of malaria (see Table 6.1).

According to the 2007 National Health Plan, in 2009 international NGOs and other aid agencies contributed up to 72 percent in direct support to government health facilities in terms of medical supplies, equipment, and staff training, which is why the 2010-15 NMSP indicates that "the most immediate challenge" for healthcare services in Liberia "is expanding access to basic health care of acceptable quality, with a health facility within $10 \mathrm{~km}$ ” (NMSP 10). To achieve this goal, funding must be provided at the county level, and essential medicines and other health commodities must be available to support the uninterrupted delivery of basic services to the people (NMSP 11). 
For health-risk-message design, the NMCP Report indicates that "advocacy and behavioral change communication” remain its major tools for communicating healthrisk messages to the target population. However, the NMCP Report adds that because the use of ACT, indoor residual spraying, and related intervention services in Liberia is "still low... [and] more needs to be done both by the MOH\&SW and her partners in terms of behavior change communication, if Liberia is to achieve the WHO/RBM [Roll Back Malaria] targets of reducing malaria morbidity and mortality by 50 percent by the year 2010 [now modified with the new target year of 2015].” Certainly, more needs to be done in terms of risk management and risk-communication-information design

\begin{tabular}{|c|c|c|c|}
\hline \multicolumn{4}{|c|}{ Table 6.1 Sample Bed Nets Distributed by NMCP Across Liberia in 2009} \\
\hline Quantity & $\begin{array}{c}\text { Recipient } \\
\text { Communities or } \\
\text { Counties }\end{array}$ & $\begin{array}{c}\text { Month of } \\
\text { Distribution }\end{array}$ & Donated by \\
\hline 4,500 & $\begin{array}{l}\text { Steve Tolbert Estate, } \\
\text { Zone } 12 \text { and Watch } \\
\text { Tower Communities in } \\
\text { Monrovia }\end{array}$ & Jan & UNICEF \\
\hline 194,000 & $\begin{array}{l}\text { Pregnant Women \& } \\
\text { Under } 5 \text { in Margibi and } \\
\text { Rural Mont. Todee, } \\
\text { Careysburg, St. Paul, \& } \\
\text { Common Wealth Dist.) }\end{array}$ & Feb & GFATM/UNDP \\
\hline 50,800 & $\begin{array}{l}\text { Communities in River } \\
\text { Gee, Rivercess, and } \\
\text { Sinoe Counties }\end{array}$ & May & $\begin{array}{l}\text { German } \\
\text { Government/GAA }\end{array}$ \\
\hline 450,000 & $\begin{array}{l}\text { Communities in Lofa, } \\
\text { Nimba, Grand Bassa, } \\
\text { and Montserrado }\end{array}$ & June & PMI/USAID \\
\hline 74,000 & $\begin{array}{l}\text { Communities in } \\
\text { Maryland, Grand Kru, } \\
\text { Sinoe, and River Gee } \\
\text { Counties }\end{array}$ & June thru Aug & UNICEF \\
\hline 5,000 & $\begin{array}{l}\text { HHs in Rural Mont. } \\
\text { (Careysburg Dist.) }\end{array}$ & July 2009 & $\begin{array}{l}\text { Save the } \\
\text { Children-UK }\end{array}$ \\
\hline
\end{tabular}


because as the NMCP Report acknowledges, one of the basic tools for improving the management of malaria-control activities in any country is an "accurate and reliable [malaria-indicator] database.” However, the NMCP Report also points out that the data from the current Health Management Information System (HMIS) at MOH\&SW are "not very reliable and cover only those with access to health facilities," which happens to be about half of the population. As a result, the NMCP Report says that the NMCP has had to rely on data from the biannual Liberia Malaria Indicator Survey (LMIS) and the biennial Health Facility Survey (HFS) in order to track progress of malaria-control interventions in the general population.

These are serious constraints that speak to a bloated government bureaucracy and the lack of adequately trained staff and up-to-date equipment. Effective health-risk communication is greatly impacted by these shortages of equipment and funding problems. For example, as late as 2009, only one training program existed in Liberia for physicians, and only seven training institutions existed for nurses and other health professionals (NMSP 10). The first step for the efficient treatment and control of any disease must be personnel training and concerted public-education campaigns rather than simply promoting drug-treatment options. Crucial governmental health policysuch as the choice of first-line treatment for malaria and other diseases - ought to be formulated not on the basis of humanitarian intervention and external-funding requirements, but on the basis of careful studies of the health situation in the country and pretesting of the new drugs among the target population. As both the survey respondents and interview participants have shown, behavior change is a gradual process that must take into account the sociocultural and economic concerns of the target population. The distribution of millions of ACTs and bed nets will not persuade people to abandon their cultural practices and traditions unless these people are strategically seated at the design and implementation table and have first-hand knowledge and understanding of the benefits that will accrue to them through their active participation. 


\subsection{Discussions}

One of the notable aspects of my field research in Liberia was the affinity of the interview participants for their local language, culture, and literacy practices. Some participants who spoke fairly good English—and had conversed with me in English prior to the interview - felt most comfortable answering the interview questions in their native Bassa language with the aid of an interpreter. As one participant asserted, "My language is more important to me because it is my tribe, and I understand it better.” Another participant said, "It’s important to speak in my dialect; I am a native woman." A third participant said that even as educated as he was with a high-school diploma and some college experience, "there are certain, certain things if you communicate with me, I still want to understand it in my dialect. So it is very very important to our people that you use [their] own local dialect to them so that they can be able to understand it clearly. I don't care how you speak the lowest English, the Liberian English, to them, there is a need, a need to...speak that dialect.”

This affinity for one's local language and culture was not only noticeable among interview participants, but it is also a major theme in the works of James Paul Gee and other literacy and language scholars such, as Brian Street, Deborah Brandt, Edward Sapir, and Benjamin Whorf. Gee, for instance, argues that "language use alone is not enough” because "paradoxically put: a person can speak a language grammatically, can use the language appropriately, and still get it 'wrong'” (124). Gee's postulation is confirmed in the Bassa expression Glan-kunm mum-gar. Although it is possible for non-native speakers of Bassa to pronounce Glan-kunm mum-gar through practice, they are likely not to make any sense of its literal translation: "In the morning is man.” To the native Bassa speaker, however, the expression Glan-kunm mum-gar is clear and succinct: it prescribes a daily ritual for each Bassa man, woman, or child to make sure to eat each morning before leaving home. Glan-kunm mum-gar is not simply a Bassalanguage phrase, it is a Bassa aphorism that holds that because no one can accurately predict what might happen after leaving home in the morning, it is always a good idea to eat something or have some food in the stomach before stepping out of the home each morning. 


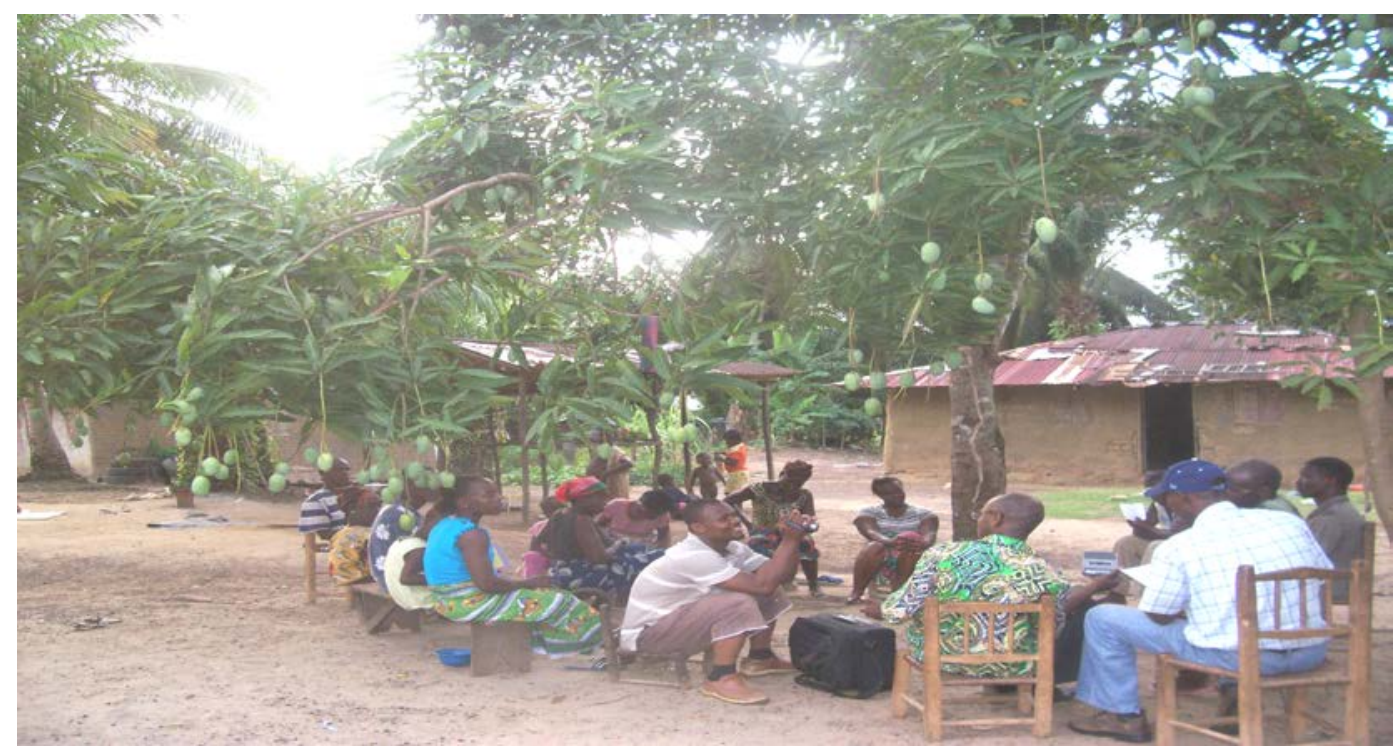

Figure 6.1: Researcher (right of briefcase) with Some Interview Participants

The non-native speaker of Bassa is not expected to understand the full meaning of such aphorisms by simply pronouncing the words or reading the literal translation of “in the morning is man.” Hence, the ability to speak a language may not be enough for one to understand that how people form "words, acts, values, beliefs, attitudes, and social identifies, as well as gestures, glances, body positions, and clothes” (Gee 127) is essential to how language is used and understood within specific contexts. In communicating health risks across diverse cultures and populations, one is also apt to understand that the ability to speak a language does not compensate for understanding the courtesies and the depth of the cultural underpinnings of that language. Benjamin Whorf suggests that Hopi language - and, hence, Hopi culture-subscribes to a metaphysical concept of time and space that is different from that of Western concepts of time and space. A similar claim might be made for Bassa and other local Liberian languages. Hence, no attempts at literal translation or coinage of an English-equivalent for Glan-kunm mum-gar and other expressions found in Bassa and other Liberian languages can compensate for the deeper concepts and beliefs underpinning such expressions and languages within their original, linguistic, cultural, and geographic settings. 
Because participation in the field interviews was voluntary, I was surprised that one participant suspended her daily chores at the local market to await my arrival, while another participant - in spite of looming deadlines to deliver clothing to her customers - placed her tailoring duties on hold as people gathered at her home to participate in the interviews. Several other people also had to suspend work in the home to participate in the interviews. However, this participation may have also been influenced by traditional Bassa cultural and literacy practices, which dictate that whenever a stranger enters town bearing a message, the most honorable and respectful thing to do is to suspend one's own activities and listen to what the stranger has to say in order to make an informed decision about accepting or rejecting the stranger's message or proposition. Hence, in the absence of the usual sounds of the traditional drum or the howls of the traditional towncrier for the people to gather at a designated point to meet with the local chief or a guest, it only took door-to-door calls or word-ofmouth messages of a single community member to summon 12 or more community members within less than 30 minutes to come and listen to me explain the purpose of my research and decide whether or not to participate (see Figure 6.1). This sort of community networking is part of traditional Bassa culture and literacy practices wherein respect for strangers outweighs the temporary inconvenience of suspending one's daily chores to listen to what the stranger has to say. Hence, as Brian Street argues, literacy practices are specific to a political or ideological context, and the consequences of such practices “vary situationally” (24). It is, however, this sort of subtlety among the Bassa people and people of other ethnic groups in Liberia that makes communicating health risks across diverse cultures and populations especially challenging, unless one is able to incorporate local culture and traditions into program design and implementation.

Although Western culture generally condones the use of explicit messages about human reproductive organs and genitalia in health-promotion campaigns, the Bassa people and people of other ethnic groups in Liberia are likely to find such messages offensive and unwelcome. As one participant argued, a word such as condom is inappropriate in Bassa language because "to talk about puberty areas is not good in [our] culture.” Another participant argued that speaking his local Bassa language 
matters most to him because "In the West, you can talk about genital areas, [but] it is a problem” to talk about genital areas in Bassa culture, which finds such talk to be offensive to the elderly and to be an attempt to pollute the minds of the youth. Hence, Street suggests that "what is often attributed to literacy per se is more often a consequence of the social conditions in which literacy is taught” (22). For the Bassa Poro and Sande institutions (traditional schools for boys and girls, respectively), literacy practices stress not just the homogeneity and unity of the community, but also utmost respect for the elderly and for the purity and uprightness of the youth.

Accordingly, we cannot speak of adapting health-risk communication to the specific cultural contexts of diverse populations without first understanding the literacy practices and linguistic cultures of the people with whom we desire to interact.

Deborah Brandt suggests that the process of literacy development and practice can be complicated to the extent that "literacy can be implicated in so many dimensions of restructuring from the social context of composing to the grounds on which competition is waged to the forms of written products themselves” (189). Given the accounts of the literary practices of people of the Vai ethnic group of Liberia, this social context wherein the restructuring of literacy practices takes place can at times be situational or geographical. In his analysis of Scribner and Cole’s research on Vai literacy in Liberia, James Paul Gee writes, “Among the Vai, literacy and schooling do not always go together. There are three sorts of literacy among the Vai, with some people having none, one, two, or all three: English literacy acquired in formal school setting; an indigenous Vai script... transmitted outside an institutional setting... and a form of literacy in Arabic” (33). For Scribner and Cole, “Knowledge of Vai script might be characterized as 'literacy without education'” (130), although they acknowledge that the "Vai script serves the bulk of personal and public needs in the villages for information preservation and communication between individuals living in different locales” (128129). Thus, given these multiple avenues for literacy development and learning in Vai society, it is often the case in Liberia that Vai who did not acquire English literacy through formal, Western-styled schooling are called "illiterates.” 
Like the Vai, three kinds of literacies can be found among the Bassa people of Grand Bassa County, where I conducted my field research: English literacy based on a Western-styled school system; literacy based on the teachings of the traditional Poro and Sande institutions; and literacy based on the Bassa Vah Script, an indigenous script which is usually taught in informal settings in private homes and churches. The Vai and Bassa ethnic groups are among five ethnic groups in Liberia with a written script for learning (see chapter 2), but due to the influence and use of English in Liberian society as a medium of instruction, public policy, and trade and commerce, these indigenous literacy practices exist only on the fringes of society within rural settings, and they exist mostly as invisible layers of society, except for a dedicated few.

Relying on the works of scholars such as Harold Schiffman, Cecil Helman, Raymond Williams, Mary Douglas, and Deborah Lupton, I argue that a society's linguistic culture is an essential medium for communicating health-risk messages within the specific cultural contexts of diverse populations. The application of linguistic culture in health-risk communication is contextualized in chapter 2, using Harold Schiffman's definition of linguistic culture as "the set of behaviors, assumptions, cultural forms, prejudices, folk belief systems, attitudes, stereotypes, ways of thinking about language, and religio-historical circumstances associated with a particular language” (5). In that chapter, I argue that as a discipline devoted to various forms of health, safety, and environmental risks, risk communication can, should, and does address a wide range of global issues, with an underlining acknowledgement that individual health-risk messages are locally produced and culturally situated and that they often reflect "the set of behaviors, assumptions, cultural forms, prejudices," etc. of specific communities. These intercepting roles of a society's literacy practices and linguistic culture form the primary pillars for communicating health risks across diverse cultures and populations. 
The literacy practices of a society, community, or ethnic group can be explored not only from the broader issues of social acceptance, intellectual respect, and cultural tolerance, but also from the perspective of not creating a new kind of literacy that will appropriate and prescribe a universal standard of literacy across all cultural and linguistic boundaries. Rather, the emphasis should be on recognizing that the practice of "literacy varies from one context to another and from one culture to another.... [as do] the effects of different literacies in different conditions” (Gee 77).

\subsubsection{Dealing with Specific Cultural Contexts}

One of participant said that communicating about health risks is vital in her community, county, and village because many people take "malaria to be a witchcraft thing." Another participant said that a need exists to canvass the community and talk to the people about health risks in the local languages and dialects: "once you want to help the person, if you meet face-to-face, from your own interaction, telling them the danger in their own local dialect, then I strongly feel that they will take that into consideration....So there's a need that our people go into the community to talk with our people. It is not only Bassa people living in Grand Bassa; there are other...peoplelike this is called Kpelle area. They can go there; the Kpelle people speak Kpelle to the people, telling them the danger of not using this net, despite it has so-so and so-so.” These responses speak directly to the complexity of communicating health risks within specific cultural contexts of diverse populations.

Professor Duane Elmer of Trinity Divinity School suggests that although every human culture is "ethnocentric," what is most important in any cross-cultural exchange is acceptance, which Elmer defines as "the ability to communicate respect and honor to the other person” (5). Elmer says that acceptance encompasses such other human qualities as openness, trust, understanding, and learning. He argues that we cannot serve people if we do not understand them, and we cannot understand them if we are not willing to learn "about them, from them, and with them" (6). Naturally, as writers and technical communicators, we cannot communicate with any audience effectively without knowing the characteristics of that audience. According to Elmer, careful 
attention to audience analysis is essential for and when communicating across diverse cultures, if we want our every encounter with the next person to be "a moment of grace, a sacred encounter whereby we express esteem and regard toward one another” (6).

Cope and Kalantzis ask this poignant question about cross-cultural encounters: "How do you create a culture of civility amongst people existing in close local and global proximity but not of the same kin group?” (137).Their simple answer to this question is to "redefine the civic in terms of pluralism" (137). They argue that because humans are cultural beings "designed in language and consciousness," we have an inclination to "combine and recombine the range of resources in the layers of [our] identity” (147) as we seek to design our personal lives. Hence, as participants in a global enterprise of increasing global partnerships and collaborations, we need more than ever before to "combine and recombine" the range of our resources to rid ourselves and our neighbors of any diseases that can pose serious challenges to our and their way of life. Implicit in Cope and Kalantzis' question is that working together in a global village - whether by choice or chance-does not preclude the notion of kinship or "kin group” interest in our interactions. And I would argue that the relative nature of culture doesn't necessarily preclude symbolic interactions between and among persons of different cultures and belief systems. Instead, what is needed is a new kind of collaborative partnership that is based on mutual respect of each other's cultures and a willingness to explore the depth of each culture and find solutions to particular problems rather than to assume that one solution is automatically preferable to all others.

Communicating health-risks and other messages across diverse cultures requires a sort of multiliteracy that is not overburdened by ethnocentrism. According to Kelli Cargile Cook, "Today, technical communicators need to be multiliterate, possessing a variety of literacies that encompass the multiple ways people use language in producing information, solving problems, and critiquing practice” (5). Cook argues that in today's world, it is incumbent upon technical communication students and practitioners to strive and to be "able to contextualize their writing, the situation in which they are writing and the concerns of all stakeholders, not just the power, who have an interest in their 
project” (16). These are challenges that also weigh heavily against adapting health-risk communication to the specific cultural contexts of diverse populations. In the process of designing a health-risk message — whatever the communicator's good intentions—-the designer and his or her culture are inseparable. Hence, individual transformations can only take place in a person who understands his or her own culture and all of the social values attached to that culture, whether by way of education or through political or religious affiliations.

MTCP administrators who responded to my survey disagreed with MTCP beneficiaries who participated in the field interviews as to how cultural adaptation could affect program outcomes. This was in part because each group had a different conception of what cultural adaption meant. For many MTCP administrators, cultural adaptation was already a burgeoning reality where traditional chiefs and elders participated in malaria-sensitization workshops as facilitators and interpreters and where some health-promotion messages were broadcasts over radio and television in selected local languages. However, for the traditional leaders and other beneficiaries of MTCPs, “cultural adaptation” meant much more than this, including a role in the distribution of bed nets and participation in policy decisions relating to the design and implementation of health-promotion messages about bed nets and related antimalarial drugs and insecticides. These differences in meaning best explain Schwandt's proposition that "We do not construct our interpretations in isolation but against a backdrop of shared understandings, practices, language, and so forth” (197).

Because a society's literacy practices and linguistic culture serve as primary pillars for adapting health-risk communication to the specific cultural contexts of diverse populations, it is essential to explore each society's physical spaces as part of health-risk-message design in order to discover and understand the perception of risk as defined within that specific community or society and how such risk information is communicated. Elizabeth Birr Moje argues that "As part of everyday practices, people use a variety of written texts and other forms of representation (i.e. oral language, dress, gestures and movements, icons, etc.) to navigate within and across physical spaces” (16). Understanding these forms of representations is crucial to effective health-risk- 
message design and implementation because, to paraphrase James Berlin's notion of ideology in composition pedagogy, the design of a health-risk message or any piece of communication is "imbricated in ideology, in a set of tacit assumptions about what is real, what is good, what is possible, and how power ought to be distributed" (697). The tacit assumptions underpinning Berlin's notion of ideology often result in an interplay of power and contesting linguistic and cultural values, beliefs, assumptions, and social spaces or “contact zones” (Pratt 34; Brizzell 738) that usually becloud any form of teaching in a cross-cultural setting, especially communication across cultures.

These "contact zones" can confound efforts to communicate across cultures, and they are often buried in the euphemism of "behavioral change communication," whereby the target audience is usually expected to accommodate and adjust to whatever changes are being recommended, in a sort of replay of the expert-layperson controversy about risk and risk perceptions in society. This sort of interaction doesn't augur well for the mutuality that should obtain in any cross-cultural communication. According to Huff and Kline, any interactions between two or more individuals "representing divergent cultural orientations and where different rules might govern the communication process, the opportunity for miscommunication is significant” (14). Hence, adapting health-risk communication to the specific cultural contexts of diverse populations requires that care be taken to establish a system of communication that is implemented "as a means of bridging the gap between individual differences and negotiating individual realities” (Kim and Gudykunst 50) within each society with respect to message clarity, coherence, and appeal to the general population.

\subsubsection{Design as Rhetoric: Toward a Rhetoric of Convergence and Cultural Accommodation}

The design of a health-risk message is rhetorical: It must have a target audience, a purpose, a context, and a focus. The length of the message and the medium through which it will be disseminated are important considerations that are often foregrounded in modes of persuasion or Aristotelian rhetorical tools, such as logos, pathos, and ethos. Because these modes of persuasion appeal to logic (logos), emotions (pathos) of the 
target audience, and to the credibility (ethos) of the message designer, it is possible to see all of these appeals in one message at the same time. As Craig Waddell suggests regarding public participation in the debates about recombinant DNA experimental research in Cambridge, Massachusetts in 1976-77, logos, pathos, and ethos often function as "complementary appeals that continually blend and interact throughout the rhetorical process” (“The Role of Pathos” 383). Waddell explains how, where, and when each of these rhetorical appeals interacts with another. The ways in which ethos and pathos interact are fundamental to effectively communicating health risk across cultures. To Waddell, “the audience’s judgment of the appropriateness of an emotional appeal may be influenced by their assessment of the speaker's ethos; conversely, the audience’s assessment of the speaker's ethos may be influenced by their assessment of the appropriateness of his or her emotional appeals” (“The Role of Pathos” 383). The ethos of the risk communicator or message designer and the pathos of the audience are essential in that a communicator's social class and his or her "prevailing ideology (outlook, values, tacit assumptions, half-realized allegiances, etc.) have a major bearing on what is written by a member of that class” (Barry 158).

Unfortunately, for much of health-risk communication and health-promotion messages, the emphasis is on behavioral change rather than on mutual exchange of ideas and information among all the participants in the communication process. Hence, the question becomes behavioral change for whom? For the consumers and not for the designers of health-risk messages? For MTCP beneficiaries and not for MTCP administrators? For national policymakers and not for the administrators and target populations involved with MTCPs? The communication process should not be encumbered by acts of social stratification; instead, it should be focused on mutuality of purpose. Therefore, notions of "behavioral change communication” should not only be for health-risk-message consumers, but also for health-risk-message designers. Hence, current emphasis on behavior-change communication should be redefined to encompass the behaviors and interactions of all the parties involved in the process, not just the beneficiaries. While behavior change is necessary for using a new product or a new drug (such as amodiaquine), it is also necessary for effective communication, mutual 
respect, cooperation, and related interactions between MTCP beneficiaries and administrators.

As Witte, Meyer, and Martell suggest, the best messages will not be effective if “inappropriate channels" are chosen for message design and dissemination (133). The consumers of health-risk messages have a sense of pride and related sensibilities and belief systems. If message designers do not share these sensibilities and belief systems, there are bound to be significant disconnects in how messages are delivered and received, including in the channels that are selected for such messages. Behaviorchange communication should never be a vehicle for suggesting that the means the people have traditionally used for generations to treat themselves for malaria and other diseases are wrong and ill-informed, and that the only "proper” means for malaria control and prevention are Western methods, such as bed nets, antimalarial drugs, and insecticides. Such a message undermines the active participation and cooperation of all stakeholders, and it is bound to generate resistance rather than the kind of mutuality that should obtain in terms of benefits to both parties.

During my field research in Liberia, a large percentage of participants used a combination of Western and traditional medicine to treat malaria; in addition, nearly all users of bed nets relied on donated nets and the technical assistance of communityhealth workers to install the nets in their homes. Some participants used the nets as decorative items in their homes, while others used the nets for fishing and other chores for which the nets were not designed. All of these scenarios are clear indicators of health-risk messages not reaching the target population effectively. For if the people clearly understood the benefits of bed nets, antimalarial drugs, and insecticides to their wellbeing, they would invest in their purchase and upkeep rather than rely on the free distributions of nets. Good intentions are not enough if those who promote the idea of bed-net use failed to demonstrate that they, too, are using bed nets at home and not just distributing them to target populations. Part of the promotion of bed-net use should include top government officials and local celebrities - such as the president, legislators, businessmen and women, and soccer stars — rather than just the current, 
small white posters being exhibited at malaria-sensitization workshops across the country.

This lack of clarity as to whether or not the promoters of bed-net use are using these nets themselves prompted a participant at the Bomi workshop to insist that those who promote bed nets should be the first to use them. If the people could see their leaders under bed nets, they would be more inclined to use the nets themselves. If people were confident that the nets can prevent malaria, they might even be willing to purchase bed nets on their own rather than depending entirely on nets that are distributed free of charge.

I do not argue for traditional medicines_-such as jologbo, ganagana, or sekou toure leaf-to replace Western medicines—such as amodiaquine, chloroquine, oral quinine, and artesume. Instead, I argue that the sociocultural conditions and practices of the people are an inherent part of their lives and must be thoroughly studied and incorporated to deliver the best treatment options available. One respondent to my survey said that local, traditional healers who claim success in treating disease should be consulted to form a part of health-risk-message design and implementation in order to find out what herbal remedies they use.

Studies of traditional medicine and traditional healers in Ghana and Tanzania suggest that some traditional medicines are, in fact, effective. In Ghana, Alex Asase and Gloria Oppong-Mensah conducted a study of traditional antimalarial-plant remedies available in herbal markets and found that "herbal medicine is important in the treatment of malaria in urban areas of Ghana where conventional drugs are not easily available” (498). In Tanzania, a study by M.C. Gessler et al. indicated that the coordination between traditional and Western medicine is still in its infancy in most countries in Africa. Nevertheless, most of the traditional healers interviewed for the study not only knew the "signs and symptoms" of malaria as defined by Western medicine, but they also were "aware of different manifestations of malaria and attributed to them different local names, which match the scientific terms which describe the different types of Plasmodium falciparurn malaria” (119). Another study in Tanzania by P. J. Winch et al. focused on the intensive reexamination of malaria-control 
strategies in Africa due both to drug resistance and recent attention to social, cultural, and behavioral responses to malaria (1057). Even in my study, respondents cherished that many of the local treatments lasted for weeks, months, and even up to two years.

Given the growing emphasis on sociocultural and behavioral responses to malaria in many communities across Africa (such as my study and the above-cited studies), what is needed is not behavior-change communication as currently structured, but a rhetorical theory of convergence and cultural accommodation that blends principles of cultural tailoring, linguistic culture, communication-accommodation theory, and community-literacy practices in designing and implementing health-risk messages adaptive to the specific cultural contexts of diverse populations. A foundational tenet of this approach is that effective health-risk communication is not about the finesse of diction or the good intentions of the communicator; instead, effective health-risk communication requires a concerted attempt to negotiate values and promote dialogue through careful identification and analysis of audience characteristics. As Brandon Johnson argues, “knowledge and ignorance exist for both laypeople and experts," so risk communicators must never fail to consider multiple dimensions of knowledge and ignorance when crafting their messages (3).

\subsection{Looking to the Future}

Many participants in my study indicated that communicating in their indigenous language is an essential component of effective health-risk communication. All human communication takes place through language (broadly defined). Our indigenous language confers on each of us legitimacy to our expressions and interactions with others and generates profound confidence in our lives. Gloria Anzaldua reflects this confidence when she says: "Until I am free to write bilingually and to switch codes without having always to translate....and as long as I have to accommodate the English speakers rather than having them accommodate me, my tongue will be illegitimate” (59). Many of the participants in the study appear to share Anzaldua's sentiments about her native language and the profound impact it has on her. 
Many of the interview participants spoke English as well as one to four local languages, but such linguistic versatility did not prevent some of them from requesting Bassa translations of interview questions and English translations of their answers. What this preference for native language shows is that many non-native speakers of English —as were all the interview participants—are not comfortable speaking English in formal settings. Yet throughout Liberian society, everyone is expected (even forced) to speak English. This illegitimates speakers of local, indigenous languages because the English speakers always want to be accommodated by the locals, but they are seldom (if ever) ready to accommodate indigenous-language speakers. But as several participants explained (and as the example of the use of Glan-kunm mum-gar illustrates), sometimes literal translations are not enough to engender understanding of a message; instead, people must understand the larger culture in order to fully comprehend a particular message.

During my field research, I also found that tremendous international goodwill exists whereby in the service of humanity, nations and organizations from the developed world are willing to uproot their people from their comfortable lives and send them into a new world of inconvenience, with degraded roads, no electricity, no safe-drinking water, and no modern, well-equipped hospitals and schools. The PMI, WHO, UNICEF, UNDP, the Global Fund, the Clinton Foundation, and others such international NGOs and aid agencies coordinate these goodwill efforts very well. However, what is often lacking is a comprehensive strategy that uses local culture and traditions in the design and implementation of MTCPs and health-risk messages to ensure the active involvement of the local people and other stakeholders in combating malaria in target populations.

I found that the healthcare-delivery system in Liberia is fragmented, underfunded, and poorly regulated and monitored. In almost every community, several, private, healthcare providers were treating patients who malaria and were selling antimalarial drugs. Hundreds of thousands dosages of ACT and hundreds of thousands of bed nets have yet to be distributed —or even accounted for — due to inadequate central storage, administrative bureaucracy, transportation, and funding . Delays in the 
distribution of bed nets and related anti-malarial drugs and insecticides have resulted in an increased used of traditional medicine, as demonstrated by the number of interview participants who used either only traditional medicine or a combination of traditional and Western medicine to treat malaria.

To resolve these problems and close these gaps, I offer the following recommendations:

- Current health-risk-message-design-and-implementation strategies for promoting the use of bed nets, antimalarial drugs, and indoor residual spraying should be restructured to involve traditional leaders and elders.

- Current malaria-sensitization workshops should be decentralized to include designated, traditional and community leaders in each town or village who will take ownership of these workshops and educate their people about malaria and the remedies being offered.

- Bed-net-distribution centers should be established within each chiefdom or village to give local people easy access to bed nets. These centers should encourage people to buy the bed nets at a minimum price and teach them how to set up the bed nets on their own without the assistance of community-health workers.

- The trial-and-error method by which ACT was introduced into Liberia has generated a backlash against the use of ACT. To avoid repeating this problem, all new drugs to be used for malaria treatment and control should be pretested within the local population to determine their efficacy and their side effects. The government should prohibit free trials of drugs by NGOs among patients in the absence of regulatory oversight.

- Some members of Liberia's general population possess the sickle-cell traits, which may either provide immunity to malaria or cause severe reaction from the use of powerful malarial drugs. Hence, a study should be conducted to ascertain how many people in the general population possess such traits and how might future dosages of antimalarial and other drugs should be administered so as to avoid unnecessary adverse reactions. 
- Because the majority of my research participants showed a strong affinity for the use of their local languages in the design and implementation of health-risk messages, efforts should be made to translate future health-risk messages into at least the most widely spoken indigenous language in a given county or region. However, where this is impossible due to inadequate funding for language development and promotion, current health-risk messages on radio and television should not be limited to 30-second public service announcements (PSAs) but should also include a talk-show format where traditional leaders will be invited to discuss in local languages malaria and its implications. Townhall meetings conducted in local languages should also be held periodically in various indigenous communities outside the city centers to solicit more active participation by local people.

- Because many of my interview participants (as well as others around the country) frequently use traditional, herbal remedies to treat malaria, a qualitative research study should be conducted to ascertain the number of herbal remedies available in the country and how these remedies could be incorporated into the healthcare-delivery system.

- Because three months was not adequate to cover every aspect of incorporating culture and traditions into the design and implementation of MTCPs in Liberia (especially how MTCP beneficiaries perceived the use of bed nets and related antimalarial drugs and insecticides, an ethnographic study should be conducted to document the health practices of the Liberian people.

These recommendations are intended to sustain and expand the dialogue established by this study regarding the incorporation of local culture and traditions in the design and implementation of MTCPs and health-risk messages. Because my field research lasted for only three months, it did not provide time to observe how frequently the participants used bed nets, indoor residual spraying, and antimalarial drugs (e.g., amodiaquine, paracetemol, or oral quinine) to prevent and treat malaria. Similarly, it was difficult to observe how frequently the participants used traditional remedies (e.g., 
pou-yong-pou, jologbo, or ganagana). Hence, there is a need for a further study into the health practices of the local people regarding how they use both Western and traditional medicine to combat malaria. As another respondent suggested, any effective malariatreatment-and-control program in Liberia will have to "attack the base of the mosquitos, which is the swamp.” He said instead of spraying the swamps to destroy the mosquito breeding grounds, "We are only stopping them in the room. But they are still, they will still be coming because they will still be producing, you see. But if you attack the base that means that the production stops. But as they are producing their eggs and things, they will still have to be coming. But in the meantime the net is alright because that's the only thing that can stop the mosquito.”

Because current malaria-treatment-and-control efforts in Liberia are dominated by the use of bed nets and indoor residual spraying - and not outdoor residual spraying, as the participant suggests - there is a need to conduct a future study that will look both at how best to incorporate local culture and traditions in program design and implementation and how to introduce the use of outdoor residual spraying to stop mosquitos from producing in the swamps. Another future study of the literacy practices of the target population for bed net-distribution should be conducted to learn how people communicate and how they would like to be communicated with. I believe these studies and activities will help in designing effective health-risk messages, built around a system of collaboration that takes into account the language, culture, and traditions of the people. This approach will help the people to better understand, embrace, and act upon these messages in the fight against malaria in diverse cultures and populations. 


\section{Works Cited}

Abraham, Thomas. "Risk and Outbreak Communication: Lessons from Alternative Paradigms.” Bulletin of the World Health Organization. 2009. 87:604-607. Web. 18 July 2010.

Agency for Toxic Substances and Diseases Registry (ATSDR). Tools and Techniques for Effective Health Risk Communication. ATSDR Primer on Health Risk Communication Principles and Practices, October 1994. Web. 15 October 2009.

Ahmad, Mohd Khairie et al. "Cultural Sensitivity in Health Promotion Program: Islamic Persuasive Communication.” Conference Presentation. 6th International Conference on Communication and Mass Media, 19-22 May 2008, Athens, Greece. Web. 17 June 2010.

Alaszewski, Andy. "Risk Communication: Identifying the Importance of Social Context.” Health, Risk, \& Society. 7.2 (June 2005): 101-105. Web. 17 March 2009.

Alexander, Neal et al "Case-Control Study of Mosquito Nets Against Malaria in the Amazon Region of Colombia.” The American Journal of Tropical Medicine and Hygiene. July 2005. 73 (1): 140-148. Web. 16 December 2011.

Amodiaquine. Medindia. n.d. Web. 6 November 2011.

Andzaldua, Gloria. Borderlands La Frontera: The New Mestiza. San Francisco: Aunt Lute Books, 1987. Print.

Asase, Alex and Gloria Oppong-Mensah. “Traditional Antimalarial Phytotherapy Remedies in Herbal Markets in Southern Ghana.” Journal of Ethnopharmacology. 126.3 (2009): 492-499. Web. 23 May 2010.

Asumana, Charles. “As Liberian Language Studies Continue At Lott Carey: Elementary Students Exhibit Academic Skills in Vai.” The News (Monrovia)/Allafrica.com. 16 July 2004. Web. 27 July 2008. <allafrica.com/stories/200407160103.html>

Barry, Peter. Beginning Theory: An Introduction to Literary and Cultural Theory. $2^{\text {nd }}$ Ed. New York: Manchester University Press, 2002. Print. 
Basic Support for Institutionalizing Child Survival. "Bolstering the Healing Process:

Continuous Health Care in Liberia.” Arlington, VA: BASICS, May 2008. Web. 19 October 2009.

Beecher, Ned et al. "Risk Perception, Risk Communication, and Stakeholder Involvement for Biosolids Management and Research.” Journal of Environmental Quality. 34.1 (2005): 122-128. Web. 10 October 2010.

Bennett, Peter et al., eds. Risk Communication and Public Health, $2^{\text {nd }}$ Ed. New York: Oxford University Press, 2010. Print.

Berlin, James A. "Rhetoric and Ideology in the Writing Class.” Cross-Talk in Comp Theory. Victor Villanueva, Jr. Ed. Urbana: NCTE, 1997. Print.

Bizzell, Patricia. “'Contact Zones’ and English Studies.” In Cross-Talk in Comp Theory. Victor Villanueva, Jr. Ed. Urbana: NCTE, 1997. Print.

Bill Cope and Mary Kalantzis. Multiliteracies: The beginnings of an idea.” In Multiliteracies: Literacy Learning and the Design of Social Futures. Eds. Bill Cope and Mary Kalantzis London: Routledge, 2000. Print.

Bitzer, Lloyd F. “The Rhetorical Situation.” Philosophy and Rhetoric. 1 (January, 1968).1-14. Web. 23 July 2011.

Black, Edwin. Rhetorical Criticism: A Study In Method. Madison: U of Wisconsin Press, 1965. Print.

Brandt, Deborah. Literacy in American Lives. New York: Cambridge Univ. Press.,2001. Print.

Burke, Kenneth. A Grammar of Motives. Berkeley: University of California Press, 1945. Print.

- - -. A Rhetoric of Motives. Berkeley: University of California Press, 1969. Print.

Bush, George W. Speech by President George W. Bush on the launch of the President's Malaria Initiative (PMI). Press Release. Office of the Press Secretary. U.S. White House, Washington, DC. 30 June 2005. Web. 19 May 2009.

Carnagarajah, Suresh A. Resisting Linguistic Imperialism in English Teaching. Oxford: Oxford University Press, 1999. Print. 
Chartier, Jean and Sandra Gabler. "Risk Communication and Government: Theory and Application for the Canadian Food Inspection Agency.” Canadian Food Inspection Agency's Public and Regulatory Affairs Branch. Fall 2000. Web. 11 January 2010.

Chase, Stuart. Forward. Language, Thought, and Reality: Selected Writings of Benjamin Lee Whorf. Ed. John B. Carroll. Cambridge: M.I.T. Press, 1956. v-x. Print.

Conrad, Peter. The Sociology of Health \& Illness: Critical Perspectives, $7^{\text {th }}$ ed. New York: Worth Publishers: 2005. Print.

Cook, Kelli Cargile. "Layered Literacies: A Theoretical Frame for Technical Communication Pedagogy.” Technical Communication Quarterly. 11.1 (2002): 5-29. Print.

Coughlin, Richard M., and Charles Lockhart. "Grid-Group Theory and Political Ideology: A Consideration of Their Relative Strengths and Weaknesses for Explaining the Structures of Mass Belief Systems.” Journal of Theoretical Politics. 10.1 (1998): 33-58. Web. 2 January 2009.

Covello, V. et al. "Risk Communication, the West Nile Virus Epidemic, and BioTerrorism: Responding to the Communication Challenges Posed by the Intentional or Unintentional Release of a Pathogen in an Urban Setting.” Journal of Urban Health: Bulletin of the New York Academic of Medicine. 78.2 (June 2001): 389-391. Web. 14 February 2009.

Creswell, J.W. “Choosing Five Qualitative Approaches.” Qualitative Inquiry and Research Design. Sage. 2007. Print.

Danisch, Robert. "Political Rhetoric in a World Risk Society.” Rhetoric Society Quarterly 40.2 (2010): 172-192. Routedge. Web. Michigan Tech U. Library. 24 May 2011.

Dathura, Gatonye. “Malaria Vaccine Launch Tuesday.” Daily Nation (Nairobi)/ allfrica.com. 16 October 2011. Web. 19 October 2011 $<$ http://allfrica.com/stories/201110170572.htm> 
Davidson, Donald. “On the Very Idea of a Conceptual Scheme.” Philosophy of Language: The Big Questions. Ed. Andrea Nye. Oxford and Malden: Blackwell Publishers, 1998. Print.

Denzin, Norman K. and Lincoln, Yvonna S. "The Discipline and Practice of Qualitative Research.” Handbook of Qualitative Research, $3^{\text {rd }}$ Ed. Thousand Oaks, 2005. Print.

Derby, Stephen L. and Ralph L. Keeney. "Risk Analysis: Understanding 'How Safe Is Safe Enough?”’ Readings in Risk. Eds. Theodore S. Glickman and Michael Gough. Washington, D.C.: Resources for the Future, 1993. Print.

de Saussure, Ferdinand. "Linguistic Value." The Communication Theory Reader. New York: Routledge, 1996. Print.

Douglas, Mary. “A History of Grid and Group Cultural Theory.” Lecture. n.p., n.d. Web. 8 January 2010. $<$ http://projects.chass.utoronto.ca/semiotics/cyber/douglas1.pdf >

Dunah, Worlea-Saywah. Keynote Address by Representative Worlea-Saywah Dunah, 5th District, Nimba County, Liberia. The 14th Annual Conference of LIBTRALO. Monrovia, Liberia. 5 March 2010.

Dutta, Mohan J. Communicating Health: A Culture-Centered Approach. Cambridge, UK: Polity Press, 2008. Print.

Eastman, Carol M. Aspects of Language and Culture. Novato: Chandler and Sharp Publishers, 1980. Print.

Elmer, Duane. "How to Serve Across Cultures: An interview with Dr. Duane Elmer." Trinity Magazine. Winter 2007. Web. 2 July 2011 $<$ http://www.tiu.edu/tiu/publications/trinitymagazine/howtoserveacrosscultur es>

Environmental Protection Agency (EPA). "Chapter 29: Risk Communication.” Washington, DC: EPA, April 2004. Web. 8 February 2010. $<$ http://www.epa.gov/ttn/fera/data/risk/vol_1/chapter_29.pdf> 
Faccone, Claudia et al. "The Effects of Language on Thought.” 19 April 2000. Web. 22 May $2011<$ http://www.unc.edu/ jdumas/projects/languagethought.htm>

Farmer, Paul. Pathologies of Power: Health, Human Rights, and the New Ward on the Poor. Los Angeles: Univ. of California Press, 2003. Print.

Ferguson, Charles A. “Diaglossia.” Word, 15 (1959). 325-340. n.d., n. pag. Web. 28 May 2011.

Firestone, William A. "Meaning In Method: The Rhetoric of Quantitative and Qualitative Research.” Educational Researcher 16.7. (October 1987): 16-21. Print.

Fischhoff, Baruch. "Risk Perception and Communication Unplugged: Twenty Years of Process.” Risk Analysis. 15.2. (1995). 137-145. Web. 19 March 2009.

Fischhoff, Baruch., Stephen T. Watson, and Chris Hope. "Defining Risk.” Readings in Risk. Eds. Theodore S. Glickman and Michael Gough. Washington, D.C. Resources for the Future. 1993. Print.

Foss, Sonja K. Rhetorical Criticism: Exploration and Practice, $4^{\text {th }}$ Ed. Long Grove: Waveland Press, 2009. Print.

Finkel, Michael. "Bedlam in the Blood Malaria." National Geographic Magazine.com. July 2007. Web. 7 April 2008. $<$ http://ngm.nationalgeographic.com/ngm/0707/feature1/index.html> Fredericks, Larri and Felicia Schanche Hodge. "Traditional Approaches to Health Care Among American Indians and Alaska Natives: A Case Study.” Promoting Health in Multicultural Populations: A Handbook or Practitioners.” Eds. Robert M. Huff and Michael V. Kline. Thousands Oak: Sage, 1999. Print.

Gay, R. L. and Peter Airasian. Educational Research:_Competencies for Analysis and Applications. $7^{\text {th }}$ Ed. Columbus: Merrill Publishing, 2003. Print.

Gee, James Paul. Social Linguistics and Literacies: Ideology in Discourses, $2^{\text {nd }}$ Ed. New York. Routledge, 1996. Print.

Gessler, M.C. et al. "Traditional Healers in Tanzania: The Perception of Malaria and Its Causes.” Journal of Ethnopharmacology. 48.3 (Nov. 1995): 119-30. Web. 14 January 2009. 
Global Health Council. Fact Sheet: “Malaria Eradication: Is it Possible?” Washington, DC: GHC, June 2009. Web. 14 July 2010.

Glickman, Theodore S. and Michael Gough. Eds. Readings in Risk. Washington, DC: Resources for the Future, $3^{\text {rd }}$ Ed. 1993. Print.

Grabill, Jeffrey T. and W. Michele Simmons. "Toward a Critical Rhetoric of Risk Communication: Producing Citizens and the Role of Technical Communication.'” Technical Communication Quarterly 7.4 (1998): 425. Print. Grin, Francois. "Economic Considerations in Language Policy.” An Introduction to Language Policy: Theory and Method. Ed. Thomas Ricento. Malden: Blackwell Publishing, 2006. Print.

Hahn, Robert A and Marcia C. Inhorn. Anthropology and Public Health: Bridging Differences in Culture and Society. $2^{\text {nd }}$ Ed. New York: Oxford University Press, 2009. Print.

Heidegger, Martin. On The Way To Language. New York: Harper and Row, 1971. Print.

Helman, Cecil G. Culture, Health and Illness, 4th Ed. London: Butterworth Heinemann. 2000. Print.

Horner, Jennifer R. "Using Culture-Centered Qualitative Formative Research to Design Broadcast Messages for HIV Prevention for African American Adolescents.” Journal of Health Communication: International Perspectives. Volume 13, Issue 4, 2008, 309 - 325. Web. 31 May 2011.

Huff, Robert M. and Michael V. Kline. Promoting Health in Multicultural Populations: A Handbook for Practitioners. Thousand Oaks: Sage Publications, 1999. Print. Institute of Statistics and Geo-Information Services et al. 2007 Demographic and Health Survey (LDHS). Monrovia: LISGIS, 2008. Web. 19 August 2010. Johnson, Branden B. “Advancing Understanding of Knowledge's Role in Lay Risk Perception.” Risk, 4.189 (Summer 1993): N. pag. Franklin Pierce Law Center, University of New Hempshire. Web. 5 April 2010. $<$ http://law.unh.edu/risk/vol4/summer/johnson.htm> 
Kamat, Vinay R. “The Anthropology of Childhood Malaria in Tanzania.” In Anthropology and Public Health: Bridging Differences in Culture and Society. $2^{\text {nd }}$ Ed. Robert A Hahn and Marcia C. Inhorn. New York: Oxford University Press, 2009. Print.

Kim, Yun Young and William B. Gudykunst, eds. Theories in Intercultural Communication: International and Intercultural Communication Annual Volume XII. Newbury Part: Sage, 1988. Print.

Kinneavy, James L. A Theory of Discourse. New York. Prentice-Hall, 1971. Print. Kneupper, Charles W. "Dramatistic Invention: The Pentad as a Heuristic Procedure.” Rhetoric Society Quarterly 9.3 (Summer, 1979): 130-136. JSTOR Web. Michigan Tech U Library. 7 October 2011.

Lewis, M. Paul, Ed. Ethnologue: Languages of the World. $16^{\text {th }}$ Ed. Dallas: SIL International, 2009. Web. 7 July $2010<$ http://www.ethnologue.com/>. Liberian History, Education, and Development, Inc. Press Release. Greensboro: LIHEDE, 2005. Web. 18 August 2008.

Littlejohn, Stephen W. Theories of Human Communication, $3^{\text {rd }}$ Ed. Belmont: Wadsworth Publishing, 1989. Print.

Lundgren, Regina E. and Andrea H. McMakin. Risk Communication: A Handbook for Communicating Environmental, Safety, and Health Risks. $4^{\text {th }}$ Ed. Piscataway: John Wiley and Sons, 2009. Print.

Lunsford, Andrea. “A Few Definition of Rhetoric.” Standford University. Standford.edu. n.d. Web. 5 May 2009.

Lupton, Deborah. Risk. New York: Routledge, 1999. Print.

Lyon, Arabella. Intentions: Negotiated, Contested, and Ignored. University Park: Pennsylvania State Univ. Press, 1998. Print.

Malaria Site. "Malaria Site: Charles Louis Alphonse Laveran (1845-1922).” Dr. B.S. Kakkilaya's Malaria Web Site. 14 April 2006. Web. 5 May 2010 $<$ http://www.malariasite.com/malaria/laveran.htm>

Madison, D.S. “Interviewing and Field Techniques.” In Critical Ethnography: Method, Ethics, and Performance. Thousand Oaks: Sage, 2005. Print. 
Marris, Claire., Ian H. Langford, and Timothy O'Riordan. “A Quantitative Test of the Cultural Theory of Risk Perceptions: Comparison with the Psychometric Paradigm.” Risk Analysis. 18.5 (October 1998): 635-647. Web. 15 December 2011.

Medicalnewstoday.com. "State of the World's Health, WHO Releases The Global Burden of Disease Study.” World Health Organization. 28 October 2008. Web. 25 December 2010 <http://www.medicalnewstoday.com/articles/127043.php>

Malaria Foundation International (MFI). “Fact Sheet Malaria.” MFI. August

2007. Web. 10 September 2009 <http://www.malaria.org/factpack.html>.

Miller, Carolyn. “A Humanistic Rationale for Technical Writing.” College English. 40.6 (Feb., 1979): 610-617. Print.

Mishler, Elliot. "Research Interviews as Speech Events.” Research Interviewing: Context and Narrative. Cambridge: Harvard University Press, 1986. Print. Moje, Elizabeth Birr. "Powerful Spaces: Tracing the Out-of School Literacy Spaces of Latino/a Youth” Spatializing Literacy Research and Practice. Eds. Kevin M. Leander and Margaret Sheehy. New York: Peter Lang Publishing, 2004. Print. Mwenesi, Halima Abdullah "Social science research in malaria prevention, management and control in the last two decades: An overview.” Acta Tropica. 95.2 (Sept., 2005:292-7. Web. 7 June 2010 <http://www.sciencedirect.com/science/article/pii/S0001706X0500149X>

National Malaria Control Program, Liberia Institute of Statistics and Geo-Information Services, and ICF Macro. Malaria Indicator Survey (MIS) 2005. Monrovia: NMCP,LISGIS, and Macro, 2005. Web. 14 December 2010.

- - -. Malaria Indicator Survey (MIS) 2009. Monrovia: NMCP,LISGIS, and Macro, 2005. Web. 14 December 2010.

National Malaria Control Program et al. National Malaria Strategic Plan for 2009-15. Monrovia: NMCP, 2009. Print.

National Malaria Control Program. Annual Report 2009. Monrovia: NMCP, 2011. Print. 
National Research Council (NRC). Improving Risk Communication. Washington, DC:

National Academy Press, 1989. Web. 19 April 2010.

NewsRx.com. "Sickle Cell Disease: Trait Offers Malaria Immunity To Children.” Science Letter (Jun 28, 2005): 1435. Web. 18 November 2011. <http://search.proquest.com/docview/209208303?accountid=28041>

Noor, Abdisalan $\mathrm{M}$ et al. "Insecticide-treated net coverage in Africa: mapping progress in 2000-07.” The Lancet 370.9592. (22 September, 2007): 1035-1039. Web 17 May 2010.

NZHerald. "US troops catch malaria in Liberia.” New Zealand Herald. 6 October 2003. Web. 18 December 2009. <http://www.nzherald.co.nz/liberia/news/article.cfm?l_id=460\&objectid=35271 $51>$

Ownby, K.K and Dune, L.S. “The Process by Which Persons With HIV-Related Peripheral Neuropath Manage Their Symptoms: A Qualitative Study.” Journal of Pain and Symptoms Management, 34.1 (2007): 48-59. Web. 4 July 2010.

Paulston, Christine Bratt and G. Richard Tucker, Eds. Sociolinguistics: The Essential Readings. Malden: Blackwell Publishing. 2003. Print.

Phillips, Steven. "World Must Retain Focus on Anti-Malaria Fight, Says Expert.” Allafrica.com. 21 April 2010. Web. 24 April 2010. $<$ http://allafrica.com/stories/201004230141.html?viewall=1>

Philipsen, Gerry. “An Ethnographic Approach to Communication Studies.” In B. Dervin, Ed. Paradigm Dialogues: Research Exemplars. Sage Publications, 1989. 258-268. Print.

Pierce, Dann A. Rhetorical Criticism and Theory in Practice. New York: McGraw-Hill, 2003. Print.

Pieterse, Jan Nederveen. Globalization and Culture: Global Mélange. Lanham: Rowman and Littlefield, 2009. Print. 
Plough, Alonzo and Sheldon Krimsky. "The Emergence of Risk Communication Studies: Social and Political Context.” In Readings in Risk. Eds. Theodore S. Glickman and Michael Gough. Washington, D.C. Resources for the Future, 1993. Print.

Renn, O. et al. "The Social Amplification of Risk: Theoretical Foundations and Empirical Applications.” Journal of Social Science Issues. 48.4 (1992): 137160. Web. 12 July 2010.

Ricento, Thomas. Ed. An Introduction to Language Policy: Theory and Method. Malden: Blackwell Publishing, 2006. Print.

Rohrmann, Bernd. "A Socio-Psychological Model for Analyzing Risk Communication Processes.” The Australasian Journal of Disaster and Trauma Studies. 2000.2 (April 2004): n. pag. Web. 3 May 2010.

Sanders, Edmund. "Malaria's Toll Fuels the Case for DDT Use in Africa." Los Angles Times. 29 May 2006. Web. 1 June 2011

Sandman, Peter. "Risk = Hazard + Outrage: Coping with Controversy about Utility Risks.” Engineering News-Record, 4 October 1999. A19-A23. November 2000. Web. 6 June 2011.

Sapir, Edward. "The Status of Linguistics as a Science.” Language. 5 (1929), 207-14. Sauer, Beverly. The Rhetoric of Risk: Technical Documentation in Hazardous Environments. Mahwah: Lawrence Erlbaum Associates, 2003. Print.

Schiffman, Harold F. Linguistic Culture and Language Policy. New York: Routledge, 1996. Print.

Schwandt, Thomas A. "Paradigms and Perspectives in Transition. Three Epistemological Stances for Qualitative Inquiry: Interpretivism, Hermeneutics, and Social Constructionism.” Eds. Norman K. Denzin and Yvonna S. Lincoln. Handbook of Qualitative Research, $3^{\text {rd }}$ Ed. Thousand Oaks: Sage, 2005. Print. Scribner, Sylvia and Michael Cole. "Unpackaging Literacy.” Literacy: A Critical Sourcebook. Eds. Ellen Cushman et al. New York: Bedford/St. Martins, 2001. Print. 
Searle, John. Intentionality: An Essay in the Philosophy of Mind. New York, Cambridge: University Press,1983. Print.

Sellnow, T. L. et al. Effective Risk Communication: A Message-Centered Approach. New York: Springer, 2009. Print.

Shah. Sonia. "In Africa, anti-malaria mosquito nets go unused by recipients." Los Angeles Times. 2 May 2010. Web. 1 June 2011 $<$ http://articles.latimes.com/2010/may/02/opinion/la-oe-shah-20100502>

Sjoberg, Lennart. "World View, Political Attitudes and Risk Perceptions.” Risk: Health, Safety \& Environment. 9 (Spring 1998): 137-152. Web. 10 December 2011.

Slack, Jennifer Daryl and J. Macgregor Wise. Culture + Technology: A Primer. New York: Peter Lang Publishing, 2005. Print.

Slovic, Paul. “Trust, Emotion, Sex, Politics, and Science: Surveying the Risk-Assessment Battlefield.” Risk Analysis, 19.4 (1999): 689-701. Web. 27 April 2009.

Slovic, Paul and Elke U. Weber. "Perception of Risk Posed by Extreme Events." Conference Paper delivered at "Risk Management Strategies in an Uncertain World.” Palisades, New York. April 12-13, 2002. Web. 10 July 2010.

Spencer, Matthew, Klaus Wachtel, and Christopher J. Howe. "The Greek Vorlage of the Syra Harclensis: A Comparative Study on Method in Exploring Textual Genealogy.” TC: A Journal of Biblical Textual Criticism. 7 (2002): n. pag. Web. 11 November 2011. <http://rosetta.reltech.org/TC/vol07/SWH2002/> Star Radio Liberia. "Traditional Chief Traces Root of Failed Projects in Liberia." Star Radio News Online. 20 January 2010. Web. 18 August 2010. $<$ www.starradio.org.lr/content/view/14510/380 >

Street, Brian. Social Literacies: Critical Approaches to Literacy in Development, Ethnography and Education. London; New York: Longman, 1995. Print. 
Sturtevant, William C. "Studies in Ethnoscience.” American Anthropologist. New Series. 66.3 (Part 2): In Transcultural Studies in Cognition. American Anthropologist Special Publication 66.3 (Part 2, June 1964). Web. 24 January 2010.

Tollefson, James W. Planning Language, Planning Inequality: Language Policy in the Community. New York: Longman, 1999. Print.

United Nations Development Program. "Consolidating Peace and National Recovery for Sustainable Development.” United Nations Assistance Framework in Liberia 2008-2012. Monrovia, UNDAF-Liberia. May 2007. Web. 9 September 2009.

USAID-CDC. President's Malaria Initiative Strategic Plan. USAID-CDC Interagency Working Group. 25 July 2005. Web. 29 September 2009.

“USAID Donates U.S. \$5000 Materials to MOH.” The Analyst (Monrovia). AllAfrica.com. 8 February 2008. Web. 9 April 2008. $<$ http://allafrica.com/stories/200804081013.html >

U.S. Agency for International Development. FAST FACTS. The President's Malaria Initiative (PMI). Washington, DC: USAID, April 2011. Web. 25 May 2011.

- - -. $\quad$ Lantos-Hyde United States Government Malaria Strategy 2009-2014. Washington, DC: USAID, 2010. Web. 10 October 2010.

- - -. The President's Malaria Initiative (PMI). Malaria Operational Plan (MOP) Liberia FY 2008. Washington, DC: USAID, 2008. Web. 25 July 2011.

- - -. The President’s Malaria Initiative (PMI). Malaria Operational Plan (MOP) Liberia FY 2009. Washington, DC: USAID, 2009. Web. 17 November 2010.

- - -. The President's Malaria Initiative (PMI). Malaria Operational Plan (MOP) Liberia FY 2011.Washington, DC: USAID, 2011. Web. 25 July 2011.

Utwent.nl. University of Twente. "Health Communication. Health Belief Model." n.d., n. pag. Web. 14 November 2010.

Veeder, Rex. "The Mysterious Barricades, Language and Its Limits by Ann E. Berthoff.” Rhetoric Review. 29. 1/2 (Autumn, 2000): 100-104. Print. 
Walsh, Fergus. "Global malaria death toll falling.” British Broadcasting Corporation (BBC). 13 December 2011. Web. 23 December . $<$ http://www.bbc.co.uk/news/health-16161907>

Waddell, Craig. “Defining Sustainable Development: A Case Study in Environmental Communication.” Technical Communication Quarterly. 4 (1995): 201-216. Web. 23 December 2011.

- - -. "Saving the Great Lakes: Public Participation in Environmental Policy.” Green Culture: Environmental Rhetoric in Contemporary America. Eds. Carl G. Herndl and Stuart C. Brown. Madison: U Wisconsin P, 1996. 141-165. Web. 13 January 2009.

- - -. “The Role of Pathos in the Decision-Making Process: A Study in the Rhetoric of Science Policy.” Quarterly Journal of Speech. 76 (1990): 381-400. Web. 27 May 2011.

Warren, Carol A.B. and Tracy X. Karner. Discovering Qualitative Methods: Field Research, Interviews and Analysis. Los Angeles: Roxbury Publishing Company, 2005. Print.

Wench et al., "Bagamoyo District, Tanzania and Its Impact on the Design of a Community-based Malaria.” Soc. Sci. Med. 42.7 (1996): 1057-1067. Web. 18 April 2009.

World Health Organization. WHO Guidelines for the Treatment of Malaria, $2^{\text {nd }}$ Ed. Geneva: WHO Press, 2010. Web. 20 May 2011. <http://whqlibdoc.who.int/publications/2010/9789241547925_eng.pdf>

- - -. “Fact sheet N94.” Media Centre. World Health Organization (WHO). May 2007. Web. 12 April 2008 <http://www.who.int/mediacentre/factsheets/fs094/en/index.html>

- - -. The Malaria Case Management Operational Manual (MCMOM). Geneva: WHO, 2009. Web. 29 December 2010.

Whorf, Benjamin Lee. “An American Indian Model of the Universe.” Philosophy of Language: The Big Questions. Ed. Andrea Nye. Oxford and Malden: Blackwell Publishers, 1998. Print. 
- - -. $\quad$ “Science and Linguistics.” Technology Review. 42.6 (1940): 229-31, 247-8. Web. 19 September 2009.

Williams, Raymond. “Culture is Ordinary.” Resources of Hope: Culture, Democracy, Socialism. Ed. Robin Gable. New York. Verso, 1989. Print.

Winkelman, Michael. Culture and Health: Applying Medical Anthropology. San Francisco: John Wiley and Sons, 2009. Print.

Witte, Kim., Gary Meyer, and Dennis Martell. Effective Health Risk Messages: Stepby-Step Guide. Thousand Oaks: Sage Publications, 2001. Print. 


\section{Appendix I: IRB Approval Letter}

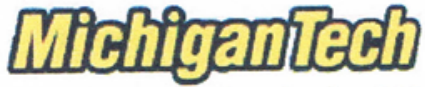 \\ Michigan Technological University
}

\section{Office of Research Integrity}

and Compliance

302 Lakeshore Center 1400 Townsend Drive Houghton, MI 49931 906.487 .2902

\section{MEMO}

TO: Dr. Craig Waddell, Humanities

CC: Nathaniel Gbessagee, Humanities

FROM: Joanne Polzien, Director Research Integrity and Compliance

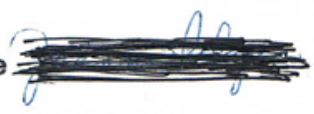

DATE: June 15, 2010

\section{SUBJECT: Approval M0574}

Your application to use human subjects in research or classroom situations has been reviewed with the following determination:

Protocol \#: M0574

Protocol Title: "Adapting Health-Risk Communication to the Specific Cultural Contexts of Diverse Populations: An Assessment of Malaria-Treatment Programs in Liberia" Approved Dates: June 15, 2010 through June 14, 2011

Approvals are granted for up to a one year period. You will need to request a continuation for each year of the project six weeks prior to the end date indicated above for each year of the project. The Office of Research Integrity and Compliance will make every effort to send the Principal Investigator annual reminders. However, the Principal Investigator is responsible for submitting annual Continuation Forms in advance of the expiration date for the project. It is very important that these expiration dates are not missed. Failure to submit annual review materials on time will result in the termination of this protocol.

This approval applies only for this project, and only under the conditions and procedures described in the application; if any changes are made in the protocol or conditions set forth in the application, the principal investigator must obtain a separate approval before these changes take place. The approved project will be subject to surveillance procedures requiring periodic review. This review will consist of consulting with the principal investigator and examining the appropriate project records.

Individual identification of human subjects in any publication is an invasion of privacy. Before beginning a project involving human subjects, and only if required, the principal investigator must obtain a properly project if a consent executed informed consent from each subject and/or the person legally responsible for the subject. If a consent form has been reviewed and approved it has been attached with an official date stamp on it. Only copies of the official date stamped informed consent is to be distributed to participants relating to this project. If any changes or modifications are needed regarding this form, you must first submit the revised document for review and approval prior to use. The principal investigator must retain informed consent forms on file at least three years after the end of the project. If a project involves a high level of risk, copies of the signed
informed consent forms must be filed with the Human Subjects Committee; if this is the case, you will be notified.

This document is on file in the Office of Research Integrity and Compliance. If you have any questions, please contact me at 487-2902 or jpolzien@mtu.edu. 


\section{Appendix 2: Survey Questions}

\section{Research Questionnaire (Category 1 Participants}

(Research Topic: “Adapting Health-Risk Communication to the Specific Cultural Contexts of Diverse Populations....”)

Affiliation Questions (Please circle answer or fill in the blank)

1) Which of the following MTCP sponsors do you work for or collaborate with?
a. WHO
b. UNMIL
c. GOL-NMCP
d. USAID
e. EU
f. Other (please specify):

2) Which of the following MTCPs do you work with?
a. PMI
b. GOL-NMCP
c. Other (please specify):

3) What is your specific role?
a. Administrator
b. Staffer
c. Designer
d. Implementer

4) How long have you worked for or collaborated with any MTCP in Liberia?
a. $0-2$ yrs.
b. 3-4 yrs.
c. 5-6 yrs.
d. 7 yrs. or more

Health-Risk Communication Questions (Please circle answer and/or fill in the blank)

The 2009-2013 National Malaria Strategic Plan for Liberia (NMSP) states that "Advocacy is an integral component of the malaria control communication strategy as public policy and social norms influence individual behavior" (NMSP 12). Please indicate the level of advocacy or health-risk communication undertaken by your agency in response to the following questions: 
5) In which language do you communicate information about MTCPs to the local population?
a. English
b. Liberian pidgin English
c. A Liberian indigenous language (please specify):
d. Other (please specify):

6) Which medium of communication do you use to share information about MTCPs? (Please circle all that apply)
a. Radio
b. Television
c. Newspaper
d. Internet
e. Text message
f. Traditional town crier
g. Public speeches
h. Other (please specify):

7) Does the design of risk-communication messages for your MTCPs include elements of the local culture and traditions?
a. Yes
b. No

8) Please explain your answer to Question \#3 with specific example(s). (Please use extra sheets if necessary):

9) Based on your answer to Question \#4, what might you do differently in the future to include local cultural values and beliefs in the design of your risk communication messages? (Please use extra sheets if necessary):

\section{Program Design \& Outcome Questions}

10) What services do your MTCPs provide? (Please circle all that apply):
a. Bed net distribution
b. Bed net use training
c. Indoor residential spraying
d. Outdoor residential spraying
e. ACT distribution
f. Public health education training
g. Other (please specify): 
11) How would you rate the services provided by your program?
a. Fair
b. Good
c. Excellent
d. Great

12) How would you rate interactions between your program and the people who benefit from the services your program provides?
a. Fair
b. Good
c. Excellent
d. Great

13) What do you believe have been the outcomes of your program in reducing malaria in Liberia?
a. Greatly successful
b. Moderately successful
c. Unsuccessful

14) Does the success rate you describe in Question \#13 fulfill or exceed the original target goals of your program?
a. Does not fulfill goals
b. Fulfills goals
c. Exceeds goals

15) Please explain (and attach supporting documentation as appropriate) what indicators you base your responses to Question \#13 and \#14 on. (Please use extra sheets if necessary):

16) Was the MTCP you work for designed inside Liberia?
a. Yes
b. No
c. Don’t Know

17) Please explain how you feel about incorporating local culture and traditions in the design and implementation of MTCPs in Liberia. (Please use extra sheet if necessary):

18) Please make any comments about MTCPs in Liberia not already covered in this questionnaire. (Please use extra sheet if necessary): 


\section{Appendix 3: Answers to Survey Questions}

\begin{tabular}{|c|c|c|c|c|}
\hline Questions & $\begin{array}{l}\text { Respondent } \\
1\end{array}$ & Respondent2 & Respondent3 & Respondent4 \\
\hline Question 1 & Who/eu & $\begin{array}{l}\text { Gol-nmcp, } \\
\text { usaid, mentor } \\
\text { initiative }\end{array}$ & $\begin{array}{l}\text { Usaid; implanting } \\
\text { partner for pmi }\end{array}$ & Gol-nmcp \\
\hline Question 2 & all & $\begin{array}{l}\text { Mentor } \\
\text { initiative }\end{array}$ & $\begin{array}{l}\text { Pmi; rebuilding } \\
\text { basic health } \\
\text { services (rbhs), ip } \\
\text { for pmi }\end{array}$ & Gol-nmcp \\
\hline Question 3 & $\begin{array}{l}\text { administrato } \\
\mathrm{r}\end{array}$ & implementer & administrator & implementer \\
\hline Question 4 & $0-2$ years & 3-4 years & $0-2$ years & 3-4 years \\
\hline Question 5 & $\begin{array}{l}\text { English/Lib } \\
\text { erian } \\
\text { language } \\
\text { unspecified }\end{array}$ & $\begin{array}{l}\text { English, } \\
\text { pidgin } \\
\text { English, } \\
\text { Bassa }\end{array}$ & $\begin{array}{l}\text { Pidgin English, } \\
\text { Bassa, Kpelle, } \\
\text { Grebo, Vai, } \\
\text { Lorma, Mandingo, } \\
\text { Gio, Mano }\end{array}$ & English \\
\hline Question 6 & $\begin{array}{l}\text { Radio, TV, } \\
\text { newspaper, } \\
\text { towncrier }\end{array}$ & $\begin{array}{l}\text { Radio, } \\
\text { awareness } \\
\text { meetings }\end{array}$ & $\begin{array}{l}\text { Radio, text } \\
\text { message, print } \\
\text { materials--posters, } \\
\text { flyers, traditional } \\
\text { council }\end{array}$ & $\begin{array}{l}\text { Newspaper and } \\
\text { public } \\
\text { speeches }\end{array}$ \\
\hline Question 7 & yes & yes & yes & yes \\
\hline
\end{tabular}




\begin{tabular}{|c|c|c|c|c|}
\hline $\begin{array}{l}\text { Question } \\
8\end{array}$ & $\begin{array}{l}\text { Cultural and } \\
\text { religious } \\
\text { practices affect } \\
\text { effective } \\
\text { communication } \\
\text { and must be } \\
\text { adequately } \\
\text { addressed }\end{array}$ & $\begin{array}{l}\text { We work } \\
\text { with and } \\
\text { through the } \\
\text { traditional } \\
\text { leaders. For } \\
\text { example, } \\
\text { using the } \\
\text { chief zoe to } \\
\text { meet with } \\
\text { his people } \\
\text { or facilitator } \\
\text { for } \\
\text { sensitizatio } \\
\text { n meetings }\end{array}$ & $\begin{array}{l}\text { The use of } \\
\text { national } \\
\text { traditional } \\
\text { council of } \\
\text { Liberia and } \\
\text { crusaders for } \\
\text { peace is a sure } \\
\text { way of } \\
\text { incorporating } \\
\text { the cultural } \\
\text { elements. } \\
\text { Moreover, all } \\
\text { produced } \\
\text { materials are } \\
\text { pre-tested twice; } \\
\text { developmental } \\
\text { and end user } \\
\text { testing. These } \\
\text { exercises is also } \\
\text { keen about } \\
\text { cultural factors }\end{array}$ & $\begin{array}{l}\text { Community } \\
\text { strategies and } \\
\text { intervention } \\
\text { management } \\
\text { shall be applied } \\
\text { where plans and } \\
\text { development } \\
\text { programs as } \\
\text { determining of } \\
\text { good hygiene } \\
\text { practices in } \\
\text { mobilizing zonal } \\
\text { heads, } \\
\text { community } \\
\text { residents, to } \\
\text { conduct zonal } \\
\text { cleanup } \\
\text { campaigns } \\
\text { covering } \\
\text { problematic areas } \\
\text { of ... }\end{array}$ \\
\hline $\begin{array}{l}\text { Question } \\
9\end{array}$ & $\begin{array}{l}\text { Include local } \\
\text { chiefs and } \\
\text { comment, and } \\
\text { involve } \\
\text { stakeholders in } \\
\text { message \& } \\
\text { material } \\
\text { development }\end{array}$ & $\begin{array}{l}\text { Planning } \\
\text { with the } \\
\text { traditional } \\
\text { leaders and } \\
\text { using some } \\
\text { of their } \\
\text { str4ategies } \\
\text { to promote } \\
\text { ownership } \\
\text { and } \\
\text { acceptance } \\
\text { of program }\end{array}$ & $\begin{array}{l}\text { One major gap } \\
\text { we've observed } \\
\text { for the past one } \\
\text { year plus is our } \\
\text { monitoring } \\
\text { mechanism to } \\
\text { establish the } \\
\text { effectiveness of } \\
\text { the traditional } \\
\text { leaders' } \\
\text { advocacy } \\
\text { approach. } \\
\text { Although we } \\
\text { conduct } \\
\text { quarterly dip- } \\
\text { stick studies, its } \\
\text { results are not } \\
\text { detail as it } \\
\text { relates to the } \\
\text { chiefs' } \\
\text { community } \\
\text { activities }\end{array}$ & $\begin{array}{l}\text { In order to track } \\
\text { down progress of } \\
\text { malaria control } \\
\text { interventions in } \\
\text { the general } \\
\text { population in } \\
\text { prevention, } \\
\text { immunization, } \\
\text { are expected to } \\
\text { be achieve. Also } \\
\text { to conduct house } \\
\text { to house } \\
\text { visitations in } \\
\text { order to evaluate } \\
\text { the needs of the } \\
\text { distribution of } \\
\text { mosquito bed } \\
\text { nets including, } \\
\text { insecticide } \\
\text { treated nets }\end{array}$ \\
\hline
\end{tabular}




\begin{tabular}{|l|l|l|l|l|}
\hline Question 10 & a-f & $\begin{array}{l}\text { a-c; e, f, and } \\
\text { case } \\
\text { management } \\
\text { training } \\
\text { prevention of } \\
\text { malaria in } \\
\text { pregnancy by } \\
\text { given } \\
\text { intermittent } \\
\text { preventive } \\
\text { treatment }\end{array}$ & $\begin{array}{l}\text { Public health } \\
\text { education } \\
\text { training; } \\
\text { promoting itn } \\
\text { use to prevent } \\
\text { malaria }\end{array}$ & $\begin{array}{l}\text { Public health } \\
\text { training/commun } \\
\text { ity strategies and } \\
\text { intervention } \\
\text { management }\end{array}$ \\
\hline Question 11 & good & good & good & god \\
\hline Question 12 & good & good & Good & excellent \\
\hline Question 13 & $\begin{array}{l}\text { Greatly } \\
\text { successful }\end{array}$ & $\begin{array}{l}\text { Moderately } \\
\text { successful }\end{array}$ & $\begin{array}{l}\text { Moderately } \\
\text { successful }\end{array}$ & $\begin{array}{l}\text { Greatly } \\
\text { successful }\end{array}$ \\
\hline Question 14 & $\begin{array}{l}\text { Fulfills } \\
\text { goals }\end{array}$ & $\begin{array}{l}\text { Does not fulfill } \\
\text { goals }\end{array}$ & $\begin{array}{l}\text { Does not fulfill } \\
\text { goals }\end{array}$ & Fulfills goals \\
\hline
\end{tabular}




\begin{tabular}{|c|c|c|c|c|}
\hline $\begin{array}{l}\text { Question } \\
15\end{array}$ & none & $\begin{array}{l}\text { National } \\
\text { indicators }\end{array}$ & $\begin{array}{l}\text { MOHSW } \\
\text { and NMCP }\end{array}$ & $\begin{array}{l}\text { Malaria, though } \\
\text { preventable and curable, } \\
\text { still remains as an } \\
\text { environmental problems } \\
\text { in many per: urban } \\
\text { centers becoming more } \\
\text { complex on a daily basis, } \\
\text { taking its greatest toll on } \\
\text { young children and } \\
\text { pregnant women. The } \\
\text { current challenge calls for } \\
\text { community residents to } \\
\text { harmonize activities to } \\
\text { promote sustainable } \\
\text { management of } \\
\text { environmental issues in } \\
\text { preventing the causes of } \\
\text { malaria }\end{array}$ \\
\hline $\begin{array}{l}\text { Question } \\
16\end{array}$ & yes & yes & yes & yes \\
\hline $\begin{array}{l}\text { Question } \\
17\end{array}$ & $\begin{array}{l}\text { Works } \\
\text { well to } \\
\text { create a } \\
\text { input }\end{array}$ & $\begin{array}{l}\text { Incorporatin } \\
\text { g local } \\
\text { culture and } \\
\text { tradition in } \\
\text { implementat } \\
\text { ion of } \\
\text { malaria } \\
\text { treatment } \\
\text { programs } \\
\text { will greatly } \\
\text { improve } \\
\text { acceptance } \\
\text { and } \\
\text { utilization of } \\
\text { services }\end{array}$ & $\begin{array}{l}\text { The culture } \\
\text { of any given } \\
\text { people is } \\
\text { unique to } \\
\text { the locality } \\
\text { and vary } \\
\text { from one } \\
\text { region to the } \\
\text { other. } \\
\text { Therefore, } \\
\text { ensure the } \\
\text { culture } \\
\text { appropriaten } \\
\text { ess of our } \\
\text { intervention } \\
\text { using iEC } \\
\text { materials } \\
\text { and } \\
\text { advocacy } \\
\text { meetings } \\
\text { using the } \\
\text { traditional } \\
\text { council was } \\
\text { in my } \\
\text { opinion } \\
\text { exceptional }\end{array}$ & $\begin{array}{l}\text { Areas assessed, identified } \\
\text { as critical matters of } \\
\text { concern in terms of } \\
\text { environmental hygiene } \\
\text { process so as to develop a } \\
\text { plan and activity with the } \\
\text { targeted residents to } \\
\text { minimize a problem of } \\
\text { house to house } \\
\text { sensitization in malarial } \\
\text { control intervention. } \\
\text { Considering the current } \\
\text { situation in relation to the } \\
\text { most significant } \\
\text { environmental problems } \\
\text { facing some Liberian } \\
\text { settlements and coastal } \\
\text { per urban situations }\end{array}$ \\
\hline
\end{tabular}




\begin{tabular}{|c|c|c|c|c|}
\hline $\begin{array}{l}\text { Question } \\
18\end{array}$ & none & $\begin{array}{l}\text { Destruction } \\
\text { of breeding } \\
\text { sites and } \\
\text { outdoor } \\
\text { spraying } \\
\text { which has } \\
\text { not stated } \\
\text { will also } \\
\text { help to } \\
\text { reduce } \\
\text { mosquito } \\
\text { thereby } \\
\text { reducing the } \\
\text { burden of } \\
\text { malaria }\end{array}$ & $\begin{array}{l}\text { That the just } \\
\text { concluded } \\
\text { exercise } \\
\text { using the } \\
\text { traditional } \\
\text { council to } \\
\text { conduct } \\
\text { advocacy } \\
\text { meetings at } \\
\text { county-level } \\
\text { should be } \\
\text { further } \\
\text { decentralize } \\
\text { d to smaller } \\
\text { sub-political } \\
\text { divisions } \\
\text { such as } \\
\text { districts and } \\
\text { major towns }\end{array}$ & $\begin{array}{l}\text { The issues raised in } \\
\text { this practical } \\
\text { approaches are not } \\
\text { exhaustive but they } \\
\text { identify a number of } \\
\text { critical factors that } \\
\text { needs to be property } \\
\text { evaluated for } \\
\text { meaningful procedure } \\
\text { in the areas of } \\
\text { education, training } \\
\text { towards behavioral } \\
\text { change in the } \\
\text { environment, as well } \\
\text { as assessment of } \\
\text { malaria treatment and } \\
\text { control programs in } \\
\text { Liberia }\end{array}$ \\
\hline
\end{tabular}




\begin{tabular}{|l|l|}
\hline Questions & Respondent21 \\
\hline Question 1 & Gol-nmcp \\
\hline Question 2 & Gol-nmcp \\
\hline Question 3 & Implementer \\
\hline Question 4 & 3-4 years \\
\hline Question 5 & English, pidgin English, and Liberian languages \\
\hline Question 6 & Radio, newspaper, internet, public speeches \\
\hline Question 7 & yes \\
\hline Question 8 & $\begin{array}{l}\text { Public health messages in pidgin English, local theater, and } \\
\text { messages in vernacular depending on region }\end{array}$ \\
\hline Question 9 & Not sure \\
\hline Question 10 & a-f \\
\hline Question 11 & excellent \\
\hline Question 12 & excellent \\
\hline Question 13 & Greatly successful \\
\hline Question 14 & Fulfills goals \\
\hline Question 15 & We have fulfilled our target goals \\
\hline Question 16 & yes \\
\hline Question 17 & $\begin{array}{l}\text { We have already included local culture and traditions our } \\
\text { interactions with the local people }\end{array}$ \\
\hline Question 18 & $\begin{array}{l}\text { The nmcp has well trained and committed staff who are } \\
\text { knowledgeable about malaria in all aspects. }\end{array}$ \\
\hline
\end{tabular}




\section{Appendix 4: Interview Questions}

\section{Health-Risk Communication and Behavior Questions}

1. What things do you consider health risks in your tribe or culture? (e.g., malaria, TB)

2. How do you treat health risks in your tribe or culture?

3. How do you communicate health risk in your tribe or culture?

4. Do you use radio, television, flyer, or drum to communicate health risks?

5. Explain some of the difficulties in communicating health risks in your tribe or culture?

6. How do the people who distribute the bed nets communicate with you?

7. Do you feel you usually get clear information on malaria treatment and health risks?

8. Whether you answer yes or no, how do you think health risks should be communicated?

9. Should your language, culture, or tradition matter in communicating health risks to you?

10. If so, why and in what ways?

a. If not, why not?

11. Do you recommend any changes in the way information about bed nets is communicated? 


\section{Demographic Questions}

12. Are you 18 or older?

13. If so, are you between ages $18-29,30-49,50-59$, or 60 and over?

14. Are you a traditional leader/elder, mother, young adult (male or female), or head of household?

15. Do you live in this city, village, or community?

16. If yes, how long have you lived in this city, village, or community?

17. Which ethnic group do you belong to?

18. Which language(s) do you speak?

19. Which religion do you belong to?

\section{Knowledge and perception of Malaria and Bed Nets Questions}

20. Have you ever heard of malaria?

21. What is the name for malaria in your local language?

22. Have you ever suffered from malaria?

23. If no, what would you do if you got malaria?

24. If yes, what kinds of treatments do take for malaria?

25. Do you use bed nets to fight off malaria?

26. If not, why not?

a. What do you use to fight off malaria?

b. Does it help?

c. How do you know?

27. If yes, how did you learn about bed nets (e.g., radio, word of mouth, health clinic)?

28. Which malaria treatment program did you get your bed nets from?

29. How long have you used bed nets?

30. Did someone teach you how to use bed nets?

31. How many people in your household use bed nets?

32. How do people in your tribe or culture feel about using bed nets?

33. How do you feel about using bed nets? 


\section{Appendix 5: Answers to Interview Questions}

\begin{tabular}{|c|c|c|c|c|c|}
\hline \# & Question & $\begin{array}{c}\text { Participant } \\
\# 1\end{array}$ & $\begin{array}{c}\text { Participant } \\
\# 2\end{array}$ & Participant \#3 & Participant \#4 \\
\hline 1. & $\begin{array}{l}\text { Are you } 18 \text { or } \\
\text { older? }\end{array}$ & Yes man & Yes woman & Yes woman & Yes male \\
\hline 2. & $\begin{array}{l}\text { If so, are you } \\
\text { between ages } 18- \\
29,30-49,50-59 \text {, } \\
\text { or } 60 \text { and over? }\end{array}$ & 63 & 39 & 60 plus & Over 60 \\
\hline 3. & $\begin{array}{l}\text { Are you traditional } \\
\text { leader/elder, } \\
\text { mother, young } \\
\text { adult (male or } \\
\text { female), or head of } \\
\text { household? }\end{array}$ & hoh & mother & mother & elder \\
\hline 4. & $\begin{array}{l}\text { Do you live in this } \\
\text { city, village, or } \\
\text { community? }\end{array}$ & $\begin{array}{l}\text { Yes,Sugarc } \\
\text { ane farm }\end{array}$ & $\begin{array}{l}\text { Sugarcane } \\
\text { farm }\end{array}$ & $\begin{array}{l}\text { No. I live in } \\
\text { Church street } \\
\text { community }\end{array}$ & $\begin{array}{l}\text { Yes, Sugarcane } \\
\text { farm }\end{array}$ \\
\hline 5. & $\begin{array}{l}\text { If yes, how long } \\
\text { have you lived in } \\
\text { this city, village, or } \\
\text { community? }\end{array}$ & 12 years & 20 years & 4 years & 30 yrs. \\
\hline 6. & $\begin{array}{l}\text { Which ethnic } \\
\text { group do you } \\
\text { belong to? }\end{array}$ & Bassa & Bassa & Kru and Bassa & kissi \\
\hline 7. & $\begin{array}{l}\text { Which language(s) } \\
\text { do you speak? }\end{array}$ & $\begin{array}{l}\text { Bassa and } \\
\text { English }\end{array}$ & Bassa & $\begin{array}{l}\text { Kru and Bassa } \\
\text { and English }\end{array}$ & $\begin{array}{l}\text { Kissi and } \\
\text { English }\end{array}$ \\
\hline 8. & $\begin{array}{l}\text { Which religion do } \\
\text { you belong to? }\end{array}$ & Christian & Christian & Christian & Christian \\
\hline 9. & $\begin{array}{l}\text { Have you ever } \\
\text { heard of malaria? }\end{array}$ & yes & yes & yes & yes \\
\hline 10 & $\begin{array}{l}\text { What is the name } \\
\text { for malaria in your } \\
\text { local language? }\end{array}$ & Sun-nee & Sun-nee & $\begin{array}{l}\text { Sun-nee (not } \\
\text { sure in Kru) }\end{array}$ & Gara tu-mon \\
\hline 11 & $\begin{array}{l}\text { Have you ever } \\
\text { suffered from } \\
\text { malaria? }\end{array}$ & yes & yes & yes & yes \\
\hline 12 & $\begin{array}{l}\text { If no, what would } \\
\text { you do if you got } \\
\text { malaria? }\end{array}$ & & & & $\begin{array}{l}\text { Bark called } \\
\text { Ganagana. } \\
\text { Some kind of } \\
\text { try in the bush. } \\
\text { Peel it and } \\
\text { break it into } \\
\text { pieces and still } \\
\text { it for } 3 \text { days and } \\
\text { drink. Take } \\
\text { malaria from } \\
\text { body. } \\
\text { Sugarcane leave } \\
\text { s and butter } \\
\text { pearl leave and } \\
\text { boil and heat } \\
\text { the body by } \\
\text { covering } \\
\text { oneself }\end{array}$ \\
\hline
\end{tabular}




\begin{tabular}{|c|c|c|c|c|c|}
\hline 13. & $\begin{array}{l}\text { If yes, what } \\
\text { kinds of } \\
\text { treatments do } \\
\text { take for } \\
\text { malaria? }\end{array}$ & $\begin{array}{l}\text { Jologbo, } \\
\text { Sekou Toure } \\
\text { leave, boil it } \\
\text { heat yourself } \\
\text { and drink it, } \\
\text { injection }\end{array}$ & $\begin{array}{l}\text { Country } \\
\text { medicine; } \\
\text { plum leave } \\
\text { mixed with } \\
\text { camphor ball }\end{array}$ & $\begin{array}{l}\text { Traditional } \\
\text { medicine. } \\
\text { Gang-son, } \\
\text { plum bark, } \\
\text { plum leave, } \\
\text { butter peal } \\
\text { leave and } \\
\text { sugarcane } \\
\text { leave. Boil } \\
\text { together and } \\
\text { keep away } \\
\text { malaria for one } \\
\text { month }\end{array}$ & \\
\hline 14. & $\begin{array}{l}\text { Do you use bed } \\
\text { nets to fight off } \\
\text { malaria? }\end{array}$ & yes & yes & yes & yes \\
\hline 15. & $\begin{array}{l}\text { If not, why } \\
\text { not? }\end{array}$ & & & & \\
\hline 16. & $\begin{array}{l}\text { What do you } \\
\text { use to fight off } \\
\text { malaria? }\end{array}$ & & & & \\
\hline 17. & Does it help? & & & & \\
\hline 18. & $\begin{array}{l}\text { How do you } \\
\text { know? }\end{array}$ & & & & \\
\hline 19. & $\begin{array}{l}\text { If yes, how did } \\
\text { you learn about } \\
\text { bed nets (i.e. } \\
\text { radio, word of } \\
\text { mouth, health } \\
\text { clinic)? }\end{array}$ & ngo & $\begin{array}{l}\text { Word of } \\
\text { mouth }\end{array}$ & $\begin{array}{l}\text { Don't wash it; } \\
\text { can itch body } \\
\text { so they wash it. }\end{array}$ & radio \\
\hline 20. & $\begin{array}{l}\text { Which malaria } \\
\text { treatment } \\
\text { program did } \\
\text { you get your } \\
\text { bed nets from? }\end{array}$ & No sure & & & merlin \\
\hline 21. & $\begin{array}{l}\text { How long have } \\
\text { you used bed } \\
\text { nets? }\end{array}$ & 2 yrs. & & 3 yrs. & 3 yrs. \\
\hline 22. & $\begin{array}{l}\text { Did someone } \\
\text { teach you how } \\
\text { to use bed } \\
\text { nets? }\end{array}$ & Yes. The ngo & $\begin{array}{l}\text { Yes; they } \\
\text { come and set } \\
\mathrm{u}\end{array}$ & & $\begin{array}{l}\text { Yes. Six } \\
\text { pieces of robe } \\
\text { that you tie } \\
\text { net to the bed } \\
\text { for use }\end{array}$ \\
\hline 23. & $\begin{array}{l}\text { How many } \\
\text { people in your } \\
\text { household use } \\
\text { bed nets? }\end{array}$ & $\begin{array}{l}\text { Use bed nets } \\
\text { and the spray. } \\
\text { The spray } \\
\text { beings in } \\
\text { mosquito } \\
\text { more }\end{array}$ & six & 3 people & \\
\hline 24. & $\begin{array}{l}\text { How do people } \\
\text { in your tribe or } \\
\text { culture feel } \\
\text { about using bed } \\
\text { nets? }\end{array}$ & Good. & alright & People like it & $\begin{array}{l}\text { Happy } \\
\text { because the } \\
\text { mosquito } \\
\text { bites and } \\
\text { prevents } \\
\text { transmitted of } \\
\text { disease }\end{array}$ \\
\hline
\end{tabular}




\begin{tabular}{|c|c|c|c|c|c|}
\hline 25. & $\begin{array}{l}\text { How do you } \\
\text { feel about } \\
\text { using bed nets? }\end{array}$ & good & alright & $\begin{array}{l}\text { Fine. If you } \\
\text { under it you } \\
\text { hot as long as } \\
\text { you under it. }\end{array}$ & $\begin{array}{l}\text { Feel happy. I } \\
\text { go under net } \\
\text { and no } \\
\text { mosquito can } \\
\text { enter to bite } \\
\text { me }\end{array}$ \\
\hline 26. & $\begin{array}{l}\text { What things do } \\
\text { you consider } \\
\text { health risks in } \\
\text { your tribe or } \\
\text { culture? (i.e. } \\
\text { malaria, TB) }\end{array}$ & Malaria, tb, & & $\begin{array}{l}\text { Cold and } \\
\text { cough }\end{array}$ & \\
\hline 27. & $\begin{array}{l}\text { How do you } \\
\text { treat health } \\
\text { risks in your } \\
\text { tribe or } \\
\text { culture? }\end{array}$ & $\begin{array}{l}\text { Take } \\
\text { traditional } \\
\text { medicine, } \\
\text { jologbo, pou- } \\
\text { yong-pou, go- } \\
\text { poe, break-it } \\
\text { and hand it up } \\
\text { in the room } \\
\text { and drives the } \\
\text { mosquito } \\
\text { away. Palm- } \\
\text { kernel some } \\
\text { drives the } \\
\text { mosquito } \\
\text { away }\end{array}$ & & & \\
\hline 28. & $\begin{array}{l}\text { How do you } \\
\text { communicate } \\
\text { health risk in } \\
\text { your tribe or } \\
\text { culture? }\end{array}$ & & $\mathrm{n} / \mathrm{a}$ & $\mathrm{n} / \mathrm{a}$ & $\begin{array}{l}\text { Word of } \\
\text { mouth }\end{array}$ \\
\hline 29. & $\begin{array}{l}\text { Do you use } \\
\text { radio, } \\
\text { television, } \\
\text { flyer, or drum } \\
\text { to } \\
\text { communicate } \\
\text { health risks? }\end{array}$ & $\begin{array}{l}\text { Send people } \\
\text { word-of- } \\
\text { mouth }\end{array}$ & Yell for help & & radio \\
\hline 30. & $\begin{array}{l}\text { Explain some } \\
\text { of the } \\
\text { difficulties in } \\
\text { communicating } \\
\text { health risks in } \\
\text { your tribe or } \\
\text { culture? }\end{array}$ & & & & \\
\hline 31. & $\begin{array}{l}\text { How do the } \\
\text { people who } \\
\text { distribute the } \\
\text { bed nets } \\
\text { communicate } \\
\text { with you? }\end{array}$ & Family head & & $\mathrm{n} / \mathrm{a}$ & No problem \\
\hline
\end{tabular}




\begin{tabular}{|c|c|c|c|c|c|}
\hline 32. & $\begin{array}{l}\text { Do you feel } \\
\text { you usually get } \\
\text { clear } \\
\text { information on } \\
\text { malaria } \\
\text { treatment and } \\
\text { health risks? } \\
\end{array}$ & & & & \\
\hline 33. & $\begin{array}{l}\text { Whether you } \\
\text { answer yes or } \\
\text { no, how do you } \\
\text { think health } \\
\text { risks should be } \\
\text { communicated? }\end{array}$ & $\begin{array}{l}\text { Radio or } \\
\text { word-of- } \\
\text { mouth }\end{array}$ & & & \\
\hline 34. & $\begin{array}{l}\text { Should your } \\
\text { language, } \\
\text { culture, or } \\
\text { tradition matter } \\
\text { in } \\
\text { communicating } \\
\text { health risks to } \\
\text { you? }\end{array}$ & $\mathrm{n} / \mathrm{a}$ & Yes, Bassa & & $\begin{array}{l}\text { Language is } \\
\text { important. } \\
\text { Preferred his } \\
\text { dialect }\end{array}$ \\
\hline 35. & $\begin{array}{l}\text { If so, why and } \\
\text { in what ways? }\end{array}$ & $\mathrm{n} / \mathrm{a}$ & $\begin{array}{l}\text { Interpret it in } \\
\text { Bassa }\end{array}$ & & \\
\hline 36. & $\begin{array}{l}\text { If not, why } \\
\text { not? }\end{array}$ & & & & \\
\hline 37. & $\begin{array}{l}\text { Do you } \\
\text { recommend } \\
\text { any changes in } \\
\text { the way } \\
\text { information } \\
\text { about bed nets } \\
\text { is } \\
\text { communicated? }\end{array}$ & no & no & $\begin{array}{l}\text { Okay with } \\
\text { current info }\end{array}$ & $\begin{array}{l}\text { Keep the net } \\
\text { clean should } \\
\text { recommend } \\
\text { that the net be } \\
\text { washed } \\
\text { before use or } \\
\text { after } \\
\text { sometime. } \\
\text { People say } \\
\text { don't watch }\end{array}$ \\
\hline
\end{tabular}




\begin{tabular}{|c|c|c|c|c|c|}
\hline$\#$ & Question & $\begin{array}{c}\text { Participant } \\
\# 5 \\
\end{array}$ & $\begin{array}{c}\text { Participa } \\
\text { nt \#6 }\end{array}$ & $\begin{array}{c}\text { Participan } \\
\text { t \#7 }\end{array}$ & $\begin{array}{c}\text { Participan } \\
\text { t \#8 }\end{array}$ \\
\hline 1. & $\begin{array}{l}\text { Are you } 18 \text { or } \\
\text { older? }\end{array}$ & Yes man & $\begin{array}{l}\text { Older } \\
\text { woman }\end{array}$ & $\begin{array}{l}\text { Older } \\
\text { woman }\end{array}$ & $\begin{array}{l}\text { Older } \\
\text { woman }\end{array}$ \\
\hline 2. & $\begin{array}{l}\text { If so, are you } \\
\text { between ages 18- } \\
29,30-49,50-59 \text {, } \\
\text { or } 60 \text { and over? }\end{array}$ & Over 60 & 29 & $30-49$ & $18-29$ \\
\hline 3. & $\begin{array}{l}\text { Are you traditional } \\
\text { leader/elder, } \\
\text { mother, young } \\
\text { adult (male or } \\
\text { female), or head of } \\
\text { household? }\end{array}$ & elder & $\begin{array}{l}\text { Female } \\
\text { young } \\
\text { adult/mot } \\
\text { her }\end{array}$ & hoh & $\begin{array}{l}\text { Mother/you } \\
\text { ng adult } \\
\text { female }\end{array}$ \\
\hline 4. & $\begin{array}{l}\text { Do you live in this } \\
\text { city, village, or } \\
\text { community? }\end{array}$ & $\begin{array}{l}\text { Yes, } \\
\text { Sugarcane } \\
\text { farm }\end{array}$ & $\begin{array}{l}\text { Yes, } \\
\text { Sugarcane } \\
\text { farm }\end{array}$ & $\begin{array}{l}\text { No. I live } \\
\text { in Sawmill }\end{array}$ & $\begin{array}{l}\text { No, Zinc } \\
\text { camp }\end{array}$ \\
\hline 5. & $\begin{array}{l}\text { If yes, how long } \\
\text { have you lived in } \\
\text { this city, village, or } \\
\text { community? }\end{array}$ & 40 yrs. & 3 yrs. & 8 yrs. & 2 yrs. \\
\hline 6. & $\begin{array}{l}\text { Which ethnic } \\
\text { group do you } \\
\text { belong to? }\end{array}$ & Bassa & Kru & Bassa & lorma \\
\hline 7. & $\begin{array}{l}\text { Which language(s) } \\
\text { do you speak? }\end{array}$ & $\begin{array}{l}\text { Bassa and } \\
\text { English }\end{array}$ & Bassa & Bassa & $\begin{array}{l}\text { Lorma, } \\
\text { Bassa, } \\
\text { English }\end{array}$ \\
\hline 8. & $\begin{array}{l}\text { Which religion do } \\
\text { you belong to? }\end{array}$ & Christian & Christian & Christian & Christian \\
\hline 9. & $\begin{array}{l}\text { Have you ever } \\
\text { heard of malaria? }\end{array}$ & yes & yes & yes & yes \\
\hline 10. & $\begin{array}{l}\text { What is the name } \\
\text { for malaria in your } \\
\text { local language? }\end{array}$ & Sun-nee & $\begin{array}{l}\text { Sun-nee } \\
\text { (Bassa) }\end{array}$ & Sun-nee & $\begin{array}{l}\begin{array}{l}\text { Sunni } \\
\text { (don't } \\
\text { know in } \\
\text { Lorma) }\end{array}\end{array}$ \\
\hline 11. & $\begin{array}{l}\text { Have you ever } \\
\text { suffered from } \\
\text { malaria? }\end{array}$ & yes & yes & yes & yes \\
\hline 12. & $\begin{array}{l}\text { If no, what would } \\
\text { you do if you got } \\
\text { malaria? }\end{array}$ & $\begin{array}{l}\text { Country } \\
\text { treatment. Put } \\
\text { leaves } \\
\text { together, boil } \\
\text { and drink. } \\
\text { Banana, } \\
\text { breadfruit } \\
\text { leave and } \\
\text { coconut bark. } \\
\text { Drink to } \\
\text { reduce } \\
\text { malaria for 2- } \\
3 \text { months } \\
\end{array}$ & & & \\
\hline
\end{tabular}




\begin{tabular}{|c|c|c|c|c|c|}
\hline 13. & $\begin{array}{l}\text { If yes, what } \\
\text { kinds of } \\
\text { treatments do } \\
\text { take for } \\
\text { malaria? }\end{array}$ & $\begin{array}{l}\text { Country } \\
\text { medicine }\end{array}$ & $\begin{array}{l}\text { Country } \\
\text { medicine } \\
\text { Lemon grass } \\
\text { and different } \\
\text { sugarcane } \\
\text { leaves boiled } \\
\text { and drink } \\
\end{array}$ & $\begin{array}{l}\text { Chlroquinn } \\
\text { e to } \\
\text { amodiaquin } \\
\text { e. Doesn't } \\
\text { like } \\
\text { traditional } \\
\text { medicine }\end{array}$ & $\begin{array}{l}\text { Drift and } \\
\text { tablet }\end{array}$ \\
\hline 14. & $\begin{array}{l}\text { Do you use bed } \\
\text { nets to fight off } \\
\text { malaria? }\end{array}$ & yes & yes & yes & no \\
\hline 15. & $\begin{array}{l}\text { If not, why } \\
\text { not? }\end{array}$ & & & & \\
\hline 16. & $\begin{array}{l}\text { What do you } \\
\text { use to fight off } \\
\text { malaria? }\end{array}$ & & & & $\begin{array}{l}\text { Mosquito } \\
\text { spray gun }\end{array}$ \\
\hline 17. & Does it help? & & & & yes \\
\hline 18. & $\begin{array}{l}\text { How do you } \\
\text { know? }\end{array}$ & & & & $\begin{array}{l}\text { By } \\
\text { spraying } \\
\text { the room } \\
\text { and closing } \\
\text { the door for } \\
\text { a few hours }\end{array}$ \\
\hline 19. & $\begin{array}{l}\text { If yes, how did } \\
\text { you learn about } \\
\text { bed nets (i.e. } \\
\text { radio, word of } \\
\text { mouth, health } \\
\text { clinic)? }\end{array}$ & $\begin{array}{l}\text { Radio and } \\
\text { from } \\
\text { community } \\
\text { event }\end{array}$ & $\begin{array}{l}\text { People came } \\
\text { around }\end{array}$ & & \\
\hline 20. & $\begin{array}{l}\text { Which malaria } \\
\text { treatment } \\
\text { program did } \\
\text { you get your } \\
\text { bed nets from? }\end{array}$ & & & $\begin{array}{l}\text { Malaria } \\
\text { control }\end{array}$ & \\
\hline 21. & $\begin{array}{l}\text { How long have } \\
\text { you used bed } \\
\text { nets? }\end{array}$ & & & $1 \mathrm{yr}$. & \\
\hline 22. & $\begin{array}{l}\text { Did someone } \\
\text { teach you how } \\
\text { to use bed } \\
\text { nets? }\end{array}$ & yes & & $\begin{array}{l}\text { Yes. Those } \\
\text { who } \\
\text { distributed } \\
\text { it }\end{array}$ & \\
\hline 23. & $\begin{array}{l}\text { How many } \\
\text { people in your } \\
\text { household use } \\
\text { bed nets? }\end{array}$ & Not sure & & nobody & none \\
\hline 24. & $\begin{array}{l}\text { How do people } \\
\text { in your tribe or } \\
\text { culture feel } \\
\text { about using bed } \\
\text { nets? }\end{array}$ & Feel good & $\begin{array}{l}\text { Feel fine. } \\
\text { When you } \\
\text { get under net } \\
\text { mosquito } \\
\text { doesn't give } \\
\text { hard time }\end{array}$ & $\begin{array}{l}\text { Many } \\
\text { people } \\
\text { don’t use } \\
\text { bed net } \\
\text { because of } \\
\text { the heat }\end{array}$ & \\
\hline
\end{tabular}




\begin{tabular}{|c|c|c|c|c|c|}
\hline 25. & $\begin{array}{l}\text { How do you } \\
\text { feel about } \\
\text { using bed } \\
\text { nets? }\end{array}$ & $\begin{array}{l}\text { Feel } \\
\text { alright. I } \\
\text { don't fight } \\
\text { mosquito } \\
\text { while } \\
\text { lying } \\
\text { under the } \\
\text { net }\end{array}$ & Feel fine. & $\begin{array}{l}\text { Feel fine but can't use it } \\
\text { because of the heat. But } \\
\text { I like to use it because I } \\
\text { don't like malaria }\end{array}$ & $\mathrm{n} / \mathrm{a}$ \\
\hline 26. & $\begin{array}{l}\text { What things } \\
\text { do you } \\
\text { consider } \\
\text { health risks in } \\
\text { your tribe or } \\
\text { culture? (i.e. } \\
\text { malaria, TB) } \\
\end{array}$ & $\begin{array}{l}\text { Tb, } \\
\text { cancer, } \\
\text { and }\end{array}$ & & $\begin{array}{l}\text { Bad luck or witchcraft; } \\
\text { some of us don’t really } \\
\text { know }\end{array}$ & Not sure \\
\hline 27. & $\begin{array}{l}\text { How do you } \\
\text { treat health } \\
\text { risks in your } \\
\text { tribe or } \\
\text { culture? } \\
\end{array}$ & $\begin{array}{l}\text { People to } \\
\text { go to } \\
\text { hospital }\end{array}$ & $\begin{array}{l}\text { Take to } \\
\text { hospital }\end{array}$ & Take to hospital & $\begin{array}{l}\text { By } \\
\text { cleaning } \\
\text { the } \\
\text { environmen } \\
\mathrm{t}\end{array}$ \\
\hline 28. & $\begin{array}{l}\text { How do you } \\
\text { communicate } \\
\text { health risk in } \\
\text { your tribe or } \\
\text { culture? }\end{array}$ & & $\begin{array}{l}\text { Tell } \\
\text { husband } \\
\text { to yell } \\
\text { for help }\end{array}$ & $\begin{array}{l}\text { Go to their house or } \\
\text { work of mouth. } \\
\text { Communicating about } \\
\text { health risk is very } \\
\text { important, especially in } \\
\text { my community, in my } \\
\text { county, and in the } \\
\text { village side because } \\
\text { people are taking } \\
\text { malaria to be a } \\
\text { witchcraft thing. Our } \\
\text { people don't believe in } \\
\text { malaria. Because, for } \\
\text { instance, when a child } \\
\text { gets malaria, I got to } \\
\text { know that malaria can } \\
\text { enter the brain through } \\
\text { radio. They can think } \\
\text { malaria is a witchcraft } \\
\text { thing. Go to the people } \\
\text { and educate them about } \\
\text { malaria because many } \\
\text { think plum, orange, } \\
\text { drink too much juice, } \\
\text { you get malaria, they } \\
\text { say when sit in the sun, } \\
\text { too much sweet bring } \\
\text { malaria }\end{array}$ & Not sure \\
\hline 29. & $\begin{array}{l}\text { Do you use } \\
\text { radio, } \\
\text { television, } \\
\text { flyer, or drum } \\
\text { to } \\
\text { communicate } \\
\text { health risks? }\end{array}$ & Towncrier & $\begin{array}{l}\text { Word of } \\
\text { mouth }\end{array}$ & $\begin{array}{l}\text { If the community uses } \\
\text { drum, she will use } \\
\text { drum, but she will use } \\
\text { phone in the city } \\
\text { modern time }\end{array}$ & Not sure \\
\hline
\end{tabular}




\begin{tabular}{|c|c|c|c|c|c|}
\hline 30. & $\begin{array}{l}\text { Explain some } \\
\text { of the } \\
\text { difficulties in } \\
\text { communicatin } \\
\text { g health risks } \\
\text { in your tribe } \\
\text { or culture? }\end{array}$ & $\begin{array}{l}\text { Some } \\
\text { people } \\
\text { don’t } \\
\text { want their } \\
\text { names to } \\
\text { be } \\
\text { exposed } \\
\text { to what } \\
\text { happened }\end{array}$ & $\begin{array}{l}\text { No } \\
\text { problem } \\
\text { because } \\
\text { it is } \\
\text { sickness }\end{array}$ & & \\
\hline 31. & $\begin{array}{l}\text { How do the } \\
\text { people who } \\
\text { distribute the } \\
\text { bed nets } \\
\text { communicate } \\
\text { with you? }\end{array}$ & & & & \\
\hline 32. & $\begin{array}{l}\text { Do you feel } \\
\text { you usually } \\
\text { get clear } \\
\text { information } \\
\text { on malaria } \\
\text { treatment and } \\
\text { health risks? }\end{array}$ & & & Clear enough & \\
\hline 33. & $\begin{array}{l}\text { Whether you } \\
\text { answer yes or } \\
\text { no, how do } \\
\text { you think } \\
\text { health risks } \\
\text { should be } \\
\text { communicate } \\
\text { d? }\end{array}$ & & & & \\
\hline 34. & $\begin{array}{l}\text { Should your } \\
\text { language, } \\
\text { culture, or } \\
\text { tradition } \\
\text { matter in } \\
\text { communicatin } \\
\text { g health risks } \\
\text { to you? }\end{array}$ & $\begin{array}{l}\text { Important } \\
\text { because } \\
\text { he will } \\
\text { understan } \\
\text { d it better }\end{array}$ & $\begin{array}{l}\text { Languag } \\
\text { e is } \\
\text { importan } \\
t\end{array}$ & Prefer Bassa & yes \\
\hline 35. & $\begin{array}{l}\text { If so, why and } \\
\text { in what ways? }\end{array}$ & $\begin{array}{l}\text { Go around } \\
\text { the town } \\
\text { to inform } \\
\text { people }\end{array}$ & $\begin{array}{l}\text { Because } \\
\text { it is good }\end{array}$ & $\begin{array}{l}\text { She understands Bassa } \\
\text { clearly so she want it in } \\
\text { Bassa }\end{array}$ & It helps \\
\hline 36. & $\begin{array}{l}\text { If not, why } \\
\text { not? }\end{array}$ & & & & \\
\hline 37. & $\begin{array}{l}\text { Do you } \\
\text { recommend } \\
\text { any changes } \\
\text { in the way } \\
\text { information } \\
\text { about bed nets } \\
\text { is } \\
\text { communicate } \\
\text { d? }\end{array}$ & $\begin{array}{l}\text { They } \\
\text { explain } \\
\text { well but } \\
\text { want } \\
\text { change. } \\
\text { Bednet } \\
\text { should be } \\
\text { a color } \\
\text { order than } \\
\text { white. }\end{array}$ & $\begin{array}{l}\text { More } \\
\text { informati } \\
\text { on. }\end{array}$ & $\begin{array}{l}\text { Info okay because they } \\
\text { do all the education-- } \\
\text { avoid dirty water, how } \\
\text { to use it, et }\end{array}$ & Not sure \\
\hline
\end{tabular}




\begin{tabular}{|c|c|c|c|c|c|}
\hline \# & Question & $\begin{array}{c}\text { Participant } \\
\# 9\end{array}$ & $\begin{array}{c}\text { Participant } \\
\# 10\end{array}$ & $\begin{array}{c}\text { Participant } \\
\# 11\end{array}$ & $\begin{array}{c}\text { Participant } \\
\text { \#12 }\end{array}$ \\
\hline 1. & $\begin{array}{l}\text { Are you } 18 \text { or } \\
\text { older? }\end{array}$ & Older male & Older woman & Older woman & 18 woman \\
\hline 2. & $\begin{array}{l}\text { If so, are you } \\
\text { between ages } 18- \\
29,30-49,50-59 \text {, } \\
\text { or } 60 \text { and over? }\end{array}$ & $30-49$ & $18-29$ & $18-29$ & $18-29$ \\
\hline 3. & $\begin{array}{l}\text { Are you } \\
\text { traditional } \\
\text { leader/elder, } \\
\text { mother, young } \\
\text { adult (male or } \\
\text { female), or head } \\
\text { of household? }\end{array}$ & Young adult & $\begin{array}{l}\text { Mother/young } \\
\text { adult }\end{array}$ & $\begin{array}{l}\text { Mother/young } \\
\text { adult }\end{array}$ & $\begin{array}{l}\text { Young adult } \\
\text { female }\end{array}$ \\
\hline 4. & $\begin{array}{l}\text { Do you live in this } \\
\text { city, village, or } \\
\text { community? }\end{array}$ & No, Sawmill & $\begin{array}{l}\text { Yes, Zinc } \\
\text { camp }\end{array}$ & $\begin{array}{l}\text { Yes, Zinc } \\
\text { camp }\end{array}$ & $\begin{array}{l}\text { No. I live in } \\
\text { Kilgore } \\
\text { town }\end{array}$ \\
\hline 5. & $\begin{array}{l}\text { If yes, how long } \\
\text { have you lived in } \\
\text { this city, village, } \\
\text { or community? }\end{array}$ & 2 yrs. & 3 yrs. & 3 yrs. & 2 yrs. \\
\hline 6. & $\begin{array}{l}\text { Which ethnic } \\
\text { group do you } \\
\text { belong to? }\end{array}$ & Bassa & Kpelle & Kpelle & $\begin{array}{l}\text { Mano (father } \\
\text { Bassa) }\end{array}$ \\
\hline 7. & $\begin{array}{l}\text { Which language(s) } \\
\text { do you speak? }\end{array}$ & $\begin{array}{l}\text { Bassa and } \\
\text { English }\end{array}$ & $\begin{array}{l}\text { Kpelle, Bassa, } \\
\text { English }\end{array}$ & $\begin{array}{l}\text { Kpelle, Bassa, } \\
\text { and English }\end{array}$ & $\begin{array}{l}\text { Bassa, } \\
\text { mano, and } \\
\text { English }\end{array}$ \\
\hline 8. & $\begin{array}{l}\text { Which religion do } \\
\text { you belong to? }\end{array}$ & Christian & Christian & Christian & Christian \\
\hline 9. & $\begin{array}{l}\text { Have you ever } \\
\text { heard of malaria? }\end{array}$ & yes & yes & yes & yes \\
\hline 10 & $\begin{array}{l}\text { What is the name } \\
\text { for malaria in your } \\
\text { local language? }\end{array}$ & Sun-nee & $\begin{array}{l}\text { Sunne (not in } \\
\text { Kpelle) }\end{array}$ & $\begin{array}{l}\text { Sun-nee (Bassa } \\
\text { not sure of } \\
\text { Kpelle) }\end{array}$ & Sun-nee \\
\hline 11 & $\begin{array}{l}\text { Have you ever } \\
\text { suffered from } \\
\text { malaria? }\end{array}$ & yes & yes & yes & yes \\
\hline 12 & $\begin{array}{l}\text { If no, what would } \\
\text { you do if you got } \\
\text { malaria? }\end{array}$ & & & & \\
\hline 13 & $\begin{array}{l}\text { If yes, what kinds } \\
\text { of treatments do } \\
\text { take for malaria? }\end{array}$ & $\begin{array}{l}\text { Traditional } \\
\text { treatment } \\
\text { jologbo, } \\
\text { tablets and } \\
\text { drafts }\end{array}$ & $\begin{array}{l}\text { Only malaria } \\
\text { tablets. cooney }\end{array}$ & $\begin{array}{l}\text { Tablets } \\
\text { coonigh }\end{array}$ & amodiaquine \\
\hline 14 & $\begin{array}{l}\text { Do you use bed } \\
\text { nets to fight off } \\
\text { malaria? }\end{array}$ & yes & $\begin{array}{l}\text { Have one but } \\
\text { can use it }\end{array}$ & $\begin{array}{l}\text { No. has one but } \\
\text { doesn't use it }\end{array}$ & $\begin{array}{l}\text { Yes but not } \\
\text { now }\end{array}$ \\
\hline 15 & If not, why not? & & & No reason & \\
\hline 16 & $\begin{array}{l}\text { What do you use } \\
\text { to fight off } \\
\text { malaria? }\end{array}$ & & $\begin{array}{l}\text { Only go to } \\
\text { hospital }\end{array}$ & nothing & \\
\hline 17 & Does it help? & & & & \\
\hline
\end{tabular}




\begin{tabular}{|c|c|c|c|c|c|}
\hline 18 & $\begin{array}{l}\text { How do you } \\
\text { know? }\end{array}$ & & & & \\
\hline 19 & $\begin{array}{l}\text { If yes, how did } \\
\text { you learn about } \\
\text { bed nets (i.e. } \\
\text { radio, word of } \\
\text { mouth, health } \\
\text { clinic)? }\end{array}$ & radio & $\begin{array}{l}\text { Bought one. } \\
\text { Over radio }\end{array}$ & $\begin{array}{l}\text { Bought one. } \\
\text { Heard over } \\
\text { radio }\end{array}$ & radio \\
\hline 20 & $\begin{array}{l}\text { Which malaria } \\
\text { treatment program } \\
\text { did you get your } \\
\text { bed nets from? }\end{array}$ & Not sure & Bought it & & merlin \\
\hline 21 & $\begin{array}{l}\text { How long have } \\
\text { you used bed nets? }\end{array}$ & 4 yrs. & $\begin{array}{l}\text { Had it for } 3 \\
\text { yrs. }\end{array}$ & $\begin{array}{l}\text { Had it for } 3 \\
\text { yrs. }\end{array}$ & 3 yrs. \\
\hline 22 & $\begin{array}{l}\text { Did someone } \\
\text { teach you how to } \\
\text { use bed nets? }\end{array}$ & & Self tied it up & No learned it. & $\begin{array}{l}\text { No } \\
\text { husband set } \\
\text { it up }\end{array}$ \\
\hline 23 & $\begin{array}{l}\text { How many people } \\
\text { in your household } \\
\text { use bed nets? }\end{array}$ & 8 people & 3 & 3 people & \\
\hline 24 & $\begin{array}{l}\text { How do people in } \\
\text { your tribe or } \\
\text { culture feel about } \\
\text { using bed nets? }\end{array}$ & $\begin{array}{l}\text { Negative } \\
\text { perception } \\
\text { that net is } \\
\text { heatly; use } \\
\text { only during } \\
\text { rainy season }\end{array}$ & $\begin{array}{l}\text { Feel fine to } \\
\text { use it because } \\
\text { when they use } \\
\text { it mosquito } \\
\text { don't hamper } \\
\text { them }\end{array}$ & $\begin{array}{l}\text { Feel fine } \\
\text { because } \\
\text { mosquito is } \\
\text { prevented }\end{array}$ & $\begin{array}{l}\text { Feel fine } \\
\text { because } \\
\text { they don’t } \\
\text { talk about } \\
\text { malaria }\end{array}$ \\
\hline 25 & $\begin{array}{l}\text { How do you feel } \\
\text { about using bed } \\
\text { nets? }\end{array}$ & $\begin{array}{l}\text { Feel find our } \\
\text { rainy season } \\
\text { but bad under } \\
\text { summer days } \\
\text { I can be } \\
\text { embarrassed } \\
\text { dry season. } \\
\text { Temperature } \\
\text { hot }\end{array}$ & $\begin{array}{l}\text { Feel fine to } \\
\text { use it } \\
\text { although she } \\
\text { hasn't used it }\end{array}$ & fine & $\begin{array}{l}\text { Feeling bad } \\
\text { because } \\
\text { malaria in } \\
\text { me, I go to } \\
\text { bed I don't } \\
\text { sleep. she } \\
\text { has } \\
\text { Monrovia } \\
\text { and doesn't } \\
\text { use bed }\end{array}$ \\
\hline 26 & $\begin{array}{l}\text { What things do } \\
\text { you consider } \\
\text { health risks in } \\
\text { your tribe or } \\
\text { culture? (i.e. } \\
\text { malaria, TB) }\end{array}$ & $\begin{array}{l}\text { Diseases such } \\
\text { as diarrhea, } \\
\text { malaria }\end{array}$ & $\begin{array}{l}\text { Flies and } \\
\text { dumpsite }\end{array}$ & $\begin{array}{l}\text { Flies, } \\
\text { dumpsite }\end{array}$ & $\begin{array}{l}\text { Dumpsite } \\
\text { right by my } \\
\text { window }\end{array}$ \\
\hline 27 & $\begin{array}{l}\text { How do you treat } \\
\text { health risks in } \\
\text { your tribe or } \\
\text { culture? }\end{array}$ & $\begin{array}{l}\text { Use tradition } \\
\text { means }\end{array}$ & & & $\begin{array}{l}\text { I don't } \\
\text { know about } \\
\text { culture } \\
\text { business } \\
\text { oo!. } \\
\end{array}$ \\
\hline 28 & $\begin{array}{l}\text { How do you } \\
\text { communicate } \\
\text { health risk in your } \\
\text { tribe or culture? }\end{array}$ & $\begin{array}{l}\text { Call } \\
\text { community } \\
\text { meeting and } \\
\text { use } \\
\text { vernacular } \\
\text { though drama }\end{array}$ & $\begin{array}{l}\text { Announce } \\
\text { over radio }\end{array}$ & radio & $\begin{array}{l}\text { Radio } \\
\text { dugbar }\end{array}$ \\
\hline
\end{tabular}




\begin{tabular}{|c|c|c|c|c|c|}
\hline 29 & $\begin{array}{l}\text { Do you use radio, } \\
\text { television, flyer, } \\
\text { or drum to } \\
\text { communicate } \\
\text { health risks? }\end{array}$ & $\begin{array}{l}\text { Sometimes } \\
\text { they } \\
\text { dramatize use } \\
\text { drama }\end{array}$ & radio & & \\
\hline 30 & $\begin{array}{l}\text { Explain some of } \\
\text { the difficulties in } \\
\text { communicating } \\
\text { health risks in } \\
\text { your tribe or } \\
\text { culture? }\end{array}$ & $\begin{array}{l}\text { Some people } \\
\text { hard to } \\
\text { understand so } \\
\text { we use drama }\end{array}$ & & & \\
\hline 31 & $\begin{array}{l}\text { How do the people } \\
\text { who distribute the } \\
\text { bed nets } \\
\text { communicate with } \\
\text { you? }\end{array}$ & $\begin{array}{l}\text { Radio and } \\
\text { meetings }\end{array}$ & Over air & & $\begin{array}{l}\text { Word of } \\
\text { mouth }\end{array}$ \\
\hline 32 & $\begin{array}{l}\text { Do you feel you } \\
\text { usually get clear } \\
\text { information on } \\
\text { malaria treatment } \\
\text { and health risks? }\end{array}$ & $\begin{array}{l}\text { Information } \\
\text { clear }\end{array}$ & & & yes \\
\hline 33 & $\begin{array}{l}\text { Whether you } \\
\text { answer yes or no, } \\
\text { how do you think } \\
\text { health risks should } \\
\text { be communicated? }\end{array}$ & $\begin{array}{l}\text { Through the } \\
\text { media radio } \\
\text { and } \\
\text { vernaculars }\end{array}$ & & & \\
\hline 34 & $\begin{array}{l}\text { Should your } \\
\text { language, culture, } \\
\text { or tradition matter } \\
\text { in communicating } \\
\text { health risks to } \\
\text { you? }\end{array}$ & seriously & $\begin{array}{l}\text { Should be in } \\
\text { my language }\end{array}$ & In language & $\begin{array}{l}\text { Yes. In } \\
\text { English }\end{array}$ \\
\hline 35 & $\begin{array}{l}\text { If so, why and in } \\
\text { what ways? }\end{array}$ & $\begin{array}{l}\text { Those in } \\
\text { hinterland to } \\
\text { understand } \\
\text { message, so } \\
\text { we put them } \\
\text { in the various } \\
\text { vernaculars . } \\
\text { going to our } \\
\text { people. }\end{array}$ & & & \\
\hline 36 & If not, why not? & & & & \\
\hline 37 & $\begin{array}{l}\text { Do you } \\
\text { recommend any } \\
\text { changes in the } \\
\text { way information } \\
\text { about bed nets is } \\
\text { communicated? }\end{array}$ & $\begin{array}{l}\text { Good } \\
\text { because they } \\
\text { did house-to- } \\
\text { house } \\
\text { mobilization' } \\
\text { come to } \\
\text { community } \\
\text { and explain }\end{array}$ & $\begin{array}{l}\text { Change to } \\
\text { spray }\end{array}$ & $\begin{array}{l}\text { Change but } \\
\text { not sure }\end{array}$ & Not sure \\
\hline
\end{tabular}




\begin{tabular}{|c|c|c|c|c|c|}
\hline$\#$ & Question & $\begin{array}{c}\text { Participant } \\
\# 13\end{array}$ & $\begin{array}{c}\text { Participant } \\
\# 14\end{array}$ & $\begin{array}{c}\text { Participant } \\
\text { \#15 }\end{array}$ & $\begin{array}{c}\text { Participant } \\
\quad \# 16\end{array}$ \\
\hline 1. & $\begin{array}{l}\text { Are you } 18 \text { or } \\
\text { older? }\end{array}$ & $\begin{array}{l}\text { Older } \\
\text { woman }\end{array}$ & $\begin{array}{l}\text { Older } \\
\text { woman }\end{array}$ & older & Older male \\
\hline 2. & $\begin{array}{l}\text { If so, are you } \\
\text { between ages } 18- \\
29,30-49,50-59 \text {, } \\
\text { or } 60 \text { and over? }\end{array}$ & $30-49$ & $18-29$ & $50-59$ & $30-49$ \\
\hline 3. & $\begin{array}{l}\text { Are you } \\
\text { traditional } \\
\text { leader/elder, } \\
\text { mother, young } \\
\text { adult (male or } \\
\text { female), or head } \\
\text { of household? }\end{array}$ & mother & Young adult & hoh & Young adult \\
\hline 4. & $\begin{array}{l}\text { Do you live in } \\
\text { this city, village, } \\
\text { or community? }\end{array}$ & $\begin{array}{l}\text { No, Zinc } \\
\text { camp }\end{array}$ & $\begin{array}{l}\text { No. I live in } \\
\text { Zinc camp }\end{array}$ & $\begin{array}{l}\text { No, } \\
\text { Fairground }\end{array}$ & $\begin{array}{l}\text { No, Tingbeh } \\
\text { town }\end{array}$ \\
\hline 5. & $\begin{array}{l}\text { If yes, how long } \\
\text { have you lived in } \\
\text { this city, village, } \\
\text { or community? }\end{array}$ & 5 yrs. & Born here & & 4 yrs. \\
\hline 6. & $\begin{array}{l}\text { Which ethnic } \\
\text { group do you } \\
\text { belong to? }\end{array}$ & Bassa & Bassa & Bassa & mano \\
\hline 7. & $\begin{array}{l}\text { Which } \\
\text { language(s) do } \\
\text { you speak? }\end{array}$ & $\begin{array}{l}\text { Bassa and } \\
\text { English }\end{array}$ & $\begin{array}{l}\text { Bassa and } \\
\text { English }\end{array}$ & $\begin{array}{l}\text { Bassa and } \\
\text { English }\end{array}$ & $\begin{array}{l}\text { English and } \\
\text { mano }\end{array}$ \\
\hline 8. & $\begin{array}{l}\text { Which religion do } \\
\text { you belong to? }\end{array}$ & Christian & Christian & Christian & Christian \\
\hline 9. & $\begin{array}{l}\text { Have you ever } \\
\text { heard of malaria? }\end{array}$ & yes & yes & yes & yes \\
\hline 10. & $\begin{array}{l}\text { What is the name } \\
\text { for malaria in } \\
\text { your local } \\
\text { language? }\end{array}$ & Sun-nee & Sun-nee & Sun-nee & nennie \\
\hline 11. & $\begin{array}{l}\text { Have you ever } \\
\text { suffered from } \\
\text { malaria? }\end{array}$ & $\begin{array}{l}\text { Yes, all the } \\
\text { time }\end{array}$ & yes & yes & yes \\
\hline 12. & $\begin{array}{l}\text { If no, what would } \\
\text { you do if you got } \\
\text { malaria? }\end{array}$ & & & & \\
\hline 13. & $\begin{array}{l}\text { If yes, what kinds } \\
\text { of treatments do } \\
\text { take for malaria? }\end{array}$ & $\begin{array}{l}\text { Chlroquinne } \\
\text { and the new } \\
\text { malaria } \\
\text { tablets. No } \\
\text { traditional } \\
\text { medicine }\end{array}$ & $\begin{array}{l}\text { Western } \\
\text { medicine } \\
\text { new malaria } \\
\text { tablet and } \\
\text { drapes }\end{array}$ & $\begin{array}{l}\text { Sometimes I } \\
\text { take tablets } \\
\text { and } \\
\text { sometime } \\
\text { country } \\
\text { medicine. } \\
\text { jologbo }\end{array}$ & $\begin{array}{l}\text { Jologbo or } \\
\text { sekou toure } \\
\text { leave and } \\
\text { Fansidar }\end{array}$ \\
\hline 14. & $\begin{array}{l}\text { Do you use bed } \\
\text { nets to fight off } \\
\text { malaria? }\end{array}$ & yes & no & yes & yes \\
\hline 15. & If not, why not? & & $\begin{array}{l}\text { Had bed net } \\
\text { but left it } \\
\text { because she } \\
\text { sleep rough }\end{array}$ & & \\
\hline
\end{tabular}




\begin{tabular}{|c|c|c|c|c|c|}
\hline 16. & $\begin{array}{l}\text { What do you use } \\
\text { to fight off } \\
\text { malaria? }\end{array}$ & & $\begin{array}{l}\text { Mosquito } \\
\text { spray }\end{array}$ & & \\
\hline 17. & Does it help? & & yes & & \\
\hline 18. & $\begin{array}{l}\text { How do you } \\
\text { know? }\end{array}$ & & $\begin{array}{l}\text { When spray } \\
\text { the mosquito } \\
\text { stops fly in } \\
\text { the room } \\
\text { until after } \\
\text { some days }\end{array}$ & & \\
\hline 19. & $\begin{array}{l}\text { If yes, how did } \\
\text { you learn about } \\
\text { bed nets (i.e. } \\
\text { radio, word of } \\
\text { mouth, health } \\
\text { clinic)? }\end{array}$ & radio & & $\begin{array}{l}\text { Health clinic } \\
\text { word of } \\
\text { mouth }\end{array}$ & $\begin{array}{l}\text { Health clinic. } \\
\text { Radio, word } \\
\text { of mouth }\end{array}$ \\
\hline 20. & $\begin{array}{l}\text { Which malaria } \\
\text { treatment } \\
\text { program did you } \\
\text { get your bed nets } \\
\text { from? }\end{array}$ & $\begin{array}{l}\text { Government } \\
\text { hospital }\end{array}$ & & merlin & $\begin{array}{l}\text { Malaria } \\
\text { control }\end{array}$ \\
\hline 21. & $\begin{array}{l}\text { How long have } \\
\text { you used bed } \\
\text { nets? }\end{array}$ & $1 \mathrm{yr}$. & & 3 yrs. & From 1990 \\
\hline 22. & $\begin{array}{l}\text { Did someone } \\
\text { teach you how to } \\
\text { use bed nets? }\end{array}$ & $\begin{array}{l}\text { Yes, the } \\
\text { hospital } \\
\text { people. } \\
\text { teach them } \\
\text { how to hang } \\
\text { it up }\end{array}$ & & $\begin{array}{l}\text { Merlin } \\
\text { taught them }\end{array}$ & $\begin{array}{l}\text { Taught him } \\
\text { how to hand it } \\
\text { on his bed }\end{array}$ \\
\hline 23. & $\begin{array}{l}\text { How many people } \\
\text { in your household } \\
\text { use bed nets? }\end{array}$ & 4 people & 2 persons & $\begin{array}{l}\text { If you have } \\
\text { four or five } \\
\text { rooms they } \\
\text { only give } \\
\text { three. } 3 \\
\text { people }\end{array}$ & 7 rooms \\
\hline 24. & $\begin{array}{l}\text { How do people in } \\
\text { your tribe or } \\
\text { culture feel about } \\
\text { using bed nets? }\end{array}$ & $\begin{array}{l}\text { Feel good } \\
\text { because he } \\
\text { net prevents } \\
\text { mosquito }\end{array}$ & $\begin{array}{l}\text { Very happy. } \\
\text { Because } \\
\text { more people } \\
\text { don't suffer } \\
\text { from malaria }\end{array}$ & $\begin{array}{l}\text { Some people } \\
\text { get used to } \\
\text { and others } \\
\text { don't. heatly }\end{array}$ & $\begin{array}{l}\text { Feel good } \\
\text { because it is } \\
\text { helpful. It's } \\
\text { important and } \\
\text { it saves from } \\
\text { malaria }\end{array}$ \\
\hline 25. & $\begin{array}{l}\text { How do you feel } \\
\text { about using bed } \\
\text { nets? }\end{array}$ & $\begin{array}{l}\text { Good and } \\
\text { bad. } \\
\text { Sometimes } \\
\text { the mosquito } \\
\text { can enter } \\
\text { and get } \\
\text { malaria. } \\
\text { Don't know } \\
\text { why. }\end{array}$ & $\begin{array}{l}\text { Feel happy } \\
\text { but stop } \\
\text { using bed } \\
\text { nets because } \\
\text { she sleep } \\
\text { rough }\end{array}$ & $\begin{array}{l}\text { Sometimes } \\
\text { don't use it } \\
\text { because it is } \\
\text { heatly }\end{array}$ & Feel good \\
\hline 26. & $\begin{array}{l}\text { What things do } \\
\text { you consider } \\
\text { health risks in } \\
\text { your tribe or } \\
\text { culture? (i.e. } \\
\text { malaria, TB) }\end{array}$ & $\begin{array}{l}\text { Dirt around } \\
\text { the house } \\
\text { and dirty } \\
\text { water in pan }\end{array}$ & $\begin{array}{l}\text { Dumpsite } \\
\text { and coach } \\
\text { roaches, } \\
\text { which bring } \\
\text { other } \\
\text { sicknesses } \\
\end{array}$ & Don’t know & Don’t know \\
\hline
\end{tabular}




\begin{tabular}{|c|c|c|c|c|c|}
\hline 27. & $\begin{array}{l}\text { How do you treat } \\
\text { health risks in } \\
\text { your tribe or } \\
\text { culture? }\end{array}$ & $\begin{array}{l}\text { Clean } \\
\text { surroundi } \\
\text { ng }\end{array}$ & $\begin{array}{l}\text { Kill it or spray } \\
\text { the room }\end{array}$ & & \\
\hline 28. & $\begin{array}{l}\text { How do you } \\
\text { communicate } \\
\text { health risk in your } \\
\text { tribe or culture? }\end{array}$ & $\begin{array}{l}\text { Radio on } \\
\text { air but not } \\
\text { sure }\end{array}$ & $\begin{array}{l}\text { Radio } \\
\text { awareness }\end{array}$ & $\begin{array}{l}\text { Word of } \\
\text { mouth }\end{array}$ & $\begin{array}{l}\text { Word of } \\
\text { mouth and } \\
\text { shout out }\end{array}$ \\
\hline 29. & $\begin{array}{l}\text { Do you use radio, } \\
\text { television, flyer, } \\
\text { or drum to } \\
\text { communicate } \\
\text { health risks? }\end{array}$ & $\begin{array}{l}\text { Tell } \\
\text { landlord } \\
\text { wife }\end{array}$ & & & \\
\hline 30. & $\begin{array}{l}\text { Explain some of } \\
\text { the difficulties in } \\
\text { communicating } \\
\text { health risks in } \\
\text { your tribe or } \\
\text { culture? }\end{array}$ & & No problem & no & \\
\hline 31. & $\begin{array}{l}\text { How do the } \\
\text { people who } \\
\text { distribute the bed } \\
\text { nets communicate } \\
\text { with you? }\end{array}$ & & $\begin{array}{l}\text { They taught } \\
\text { them how to } \\
\text { use it. They } \\
\text { informed them } \\
\text { after some days } \\
\text { they should use } \\
\text { it }\end{array}$ & $\begin{array}{l}\text { Word of } \\
\text { mouth. Use } \\
\text { community } \\
\text { sign or } \\
\text { symbols } \\
\text { using drum } \\
\text { or trumpet }\end{array}$ & $\begin{array}{l}\text { Radio to } \\
\text { inform them } \\
\text { of community } \\
\text { meeting }\end{array}$ \\
\hline 32. & $\begin{array}{l}\text { Do you feel you } \\
\text { usually get clear } \\
\text { information on } \\
\text { malaria treatment } \\
\text { and health risks? }\end{array}$ & yes & yes & & yes \\
\hline 33. & $\begin{array}{l}\text { Whether you } \\
\text { answer yes or no, } \\
\text { how do you think } \\
\text { health risks } \\
\text { should be } \\
\text { communicated? }\end{array}$ & & radio & & \\
\hline 34. & $\begin{array}{l}\text { Should your } \\
\text { language, culture, } \\
\text { or tradition matter } \\
\text { in communicating } \\
\text { health risks to } \\
\text { you? }\end{array}$ & yes & yes & yes & $\begin{array}{l}\text { Yes, It is } \\
\text { better to } \\
\text { provide } \\
\text { information in } \\
\text { his culture }\end{array}$ \\
\hline 35. & $\begin{array}{l}\text { If so, why and in } \\
\text { what ways? }\end{array}$ & $\begin{array}{l}\text { Explain in } \\
\text { my } \\
\text { language } \\
\text { and in } \\
\text { English }\end{array}$ & $\begin{array}{l}\text { It is better to } \\
\text { use traditional } \\
\text { languages } \\
\text { because many } \\
\text { of our elderly } \\
\text { people don't } \\
\text { understand } \\
\text { English. Call } \\
\text { the elder } \\
\text { people together } \\
\text { and educate } \\
\text { them }\end{array}$ & $\begin{array}{l}\text { Reason is to } \\
\text { use own } \\
\text { language } \\
\text { you know } \\
\text { better what } \\
\text { they explain. } \\
\text { You know } \\
\text { how to put } \\
\text { your own } \\
\text { language to } \\
\text { use }\end{array}$ & Not sure. \\
\hline
\end{tabular}




\begin{tabular}{|l|l|l|l|l|l|}
\hline 36. & If not, why not? & & & & \\
\hline 37. & $\begin{array}{l}\text { Do you } \\
\text { recommend any } \\
\text { changes in the } \\
\text { way information } \\
\text { about bed nets is } \\
\text { communicated? }\end{array}$ & okay & $\begin{array}{l}\text { Should be } \\
\text { changed to the } \\
\text { spraying the } \\
\text { house. Roaches } \\
\text { die and } \\
\text { mosquitos cut } \\
\text { back }\end{array}$ & $\begin{array}{l}\text { Yes. Don't } \\
\text { explain why } \\
\text { other } \\
\text { families in } \\
\text { the } \\
\text { household } \\
\text { are not given } \\
\text { bed nets }\end{array}$ & $\begin{array}{l}\text { Bednet is very } \\
\text { weak and the } \\
\text { chemical } \\
\text { burns the skin } \\
\text { without } \\
\text { burning the } \\
\text { skin. The } \\
\text { chemical } \\
\text { should be cut } \\
\text { down; maybe } \\
\text { it is too strong } \\
\text { so it's burning } \\
\text { the skin }\end{array}$ \\
\hline
\end{tabular}




\begin{tabular}{|c|c|c|c|c|c|}
\hline \# & Question & $\begin{array}{c}\text { Participant } \\
\# 17\end{array}$ & $\begin{array}{c}\text { Participant } \\
\text { \#18 }\end{array}$ & $\begin{array}{c}\text { Participant } \\
\# 19\end{array}$ & $\begin{array}{c}\text { Participant } \\
\# 20\end{array}$ \\
\hline 1. & $\begin{array}{l}\text { Are you } 18 \text { or } \\
\text { older? }\end{array}$ & Older male & Older man & Old male & Older male \\
\hline 2. & $\begin{array}{l}\text { If so, are you } \\
\text { between ages 18- } \\
29,30-49,50-59 \text {, } \\
\text { or } 60 \text { and over? }\end{array}$ & $30-49$ & $30-49$ & $30-49$ & $18-29$ \\
\hline 3. & $\begin{array}{l}\text { Are you traditional } \\
\text { leader/elder, } \\
\text { mother, young } \\
\text { adult (male or } \\
\text { female), or head of } \\
\text { household? }\end{array}$ & hoh & hoh & hoh & $\begin{array}{l}\text { Young adult } \\
\text { male }\end{array}$ \\
\hline 4. & $\begin{array}{l}\text { Do you live in this } \\
\text { city, village, or } \\
\text { community? }\end{array}$ & $\begin{array}{l}\text { No, Zinc } \\
\text { camp }\end{array}$ & $\begin{array}{l}\text { Yes, Vai } \\
\text { town }\end{array}$ & Yes, Vai town & $\begin{array}{l}\text { No. I live in the } \\
\text { Church street } \\
\text { community }\end{array}$ \\
\hline 5. & $\begin{array}{l}\text { If yes, how long } \\
\text { have you lived in } \\
\text { this city, village, or } \\
\text { community? }\end{array}$ & 3 yrs. & 10 yrs. & 30 & 4 yrs. \\
\hline 6. & $\begin{array}{l}\text { Which ethnic } \\
\text { group do you } \\
\text { belong to? }\end{array}$ & Kpelle & Kru & Bassa & Bassa \\
\hline 7. & $\begin{array}{l}\text { Which language(s) } \\
\text { do you speak? }\end{array}$ & $\begin{array}{l}\text { English and } \\
\text { Kpelle }\end{array}$ & $\begin{array}{l}\text { Kru, fanti, } \\
\text { English, } \\
\text { Bassa }\end{array}$ & $\begin{array}{l}\text { Bassa and } \\
\text { English }\end{array}$ & $\begin{array}{l}\text { Kru and } \\
\text { English }\end{array}$ \\
\hline 8. & $\begin{array}{l}\text { Which religion do } \\
\text { you belong to? }\end{array}$ & Christian & Christian & Christian & Christian \\
\hline 9. & $\begin{array}{l}\text { Have you ever } \\
\text { heard of malaria? }\end{array}$ & yes & yes & yes & yes \\
\hline 10 & $\begin{array}{l}\text { What is the name } \\
\text { for malaria in your } \\
\text { local language? }\end{array}$ & Famine & Clan-glen & Sun-nee & Clan-clen \\
\hline 11 & $\begin{array}{l}\text { Have you ever } \\
\text { suffered from } \\
\text { malaria? }\end{array}$ & yes & yes & yes & yes \\
\hline 12 & $\begin{array}{l}\text { If no, what would } \\
\text { you do if you got } \\
\text { malaria? }\end{array}$ & & & & \\
\hline 13 & $\begin{array}{l}\text { If yes, what kinds } \\
\text { of treatments do } \\
\text { take for malaria? }\end{array}$ & $\begin{array}{l}\text { Country } \\
\text { medicine and } \\
\text { western; } \\
\text { ganagana } \\
\text { (bitter), and } \\
\text { tablets }\end{array}$ & $\begin{array}{l}\text { Country } \\
\text { medicine. } \\
\text { Tablet } \\
\text { doesn't } \\
\text { work. } \\
\text { Centrine, } \\
\text { paracetemol; } \\
\text { pauper } \\
\text { leaves, } \\
\text { butter pearl } \\
\text { leave }\end{array}$ & $\begin{array}{l}\text { Combination. } \\
\text { Pauper leave, } \\
\text { breadfruit } \\
\text { leave, } \\
\text { sugarcane and } \\
\text { caforbal. Take } \\
\text { chloroquine } \\
\text { and asa }\end{array}$ & $\begin{array}{l}\text { Combination. } \\
\text { Use butter pearl } \\
\text { leave, fever } \\
\text { leave. And } \\
\text { paracetemol }\end{array}$ \\
\hline 14 & $\begin{array}{l}\text { Do you use bed } \\
\text { nets to fight off } \\
\text { malaria? }\end{array}$ & yes & yes & yes & yes \\
\hline
\end{tabular}




\begin{tabular}{|c|c|c|c|c|c|}
\hline 15 & If not, why not? & & & & \\
\hline 16 & $\begin{array}{l}\text { What do you use to } \\
\text { fight off malaria? }\end{array}$ & & & & \\
\hline 17 & Does it help? & & & & \\
\hline 18 & $\begin{array}{l}\text { How do you } \\
\text { know? }\end{array}$ & & & & \\
\hline 19 & $\begin{array}{l}\text { If yes, how did you } \\
\text { learn about bed } \\
\text { nets (i.e. radio, } \\
\text { word of mouth, } \\
\text { health clinic)? }\end{array}$ & $\begin{array}{l}\text { Radio, health } \\
\text { clinic }\end{array}$ & $\begin{array}{l}\text { Radio and } \\
\text { health clinic } \\
\text { when they } \\
\text { came to } \\
\text { share it }\end{array}$ & $\begin{array}{l}\text { Radio and } \\
\text { word of } \\
\text { mouth }\end{array}$ & $\begin{array}{l}\text { Radio and word } \\
\text { of mouth }\end{array}$ \\
\hline 20 & $\begin{array}{l}\text { Which malaria } \\
\text { treatment program } \\
\text { did you get your } \\
\text { bed nets from? }\end{array}$ & $\begin{array}{l}\text { Malaria } \\
\text { control }\end{array}$ & $\begin{array}{l}\text { Government } \\
\text { hospital }\end{array}$ & $\begin{array}{l}\text { Malaria } \\
\text { control }\end{array}$ & $\begin{array}{l}\text { Governme4nt } \\
\text { hospital }\end{array}$ \\
\hline 21 & $\begin{array}{l}\text { How long have } \\
\text { you used bed nets? }\end{array}$ & 5 yrs. & From 2000 & 6 yrs. & 4 yrs. \\
\hline 22 & $\begin{array}{l}\text { Did someone teach } \\
\text { you how to use bed } \\
\text { nets? }\end{array}$ & yes & yes & $\begin{array}{l}\text { Yes; nmcp } \\
\text { taught them }\end{array}$ & yes \\
\hline 23 & $\begin{array}{l}\text { How many people } \\
\text { in your household } \\
\text { use bed nets? }\end{array}$ & 3 & 5 & 1 & 5 \\
\hline 24 & $\begin{array}{l}\text { How do people in } \\
\text { your tribe or } \\
\text { culture feel about } \\
\text { using bed nets? }\end{array}$ & $\begin{array}{l}\text { Like it. } \\
\text { Because it } \\
\text { keeps the } \\
\text { mosquito } \\
\text { from touching } \\
\text { the body }\end{array}$ & $\begin{array}{l}\text { Most people } \\
\text { feel } \\
\text { comfortable } \\
\text { but some } \\
\text { people don't } \\
\text { like it } \\
\text { because of } \\
\text { the heat }\end{array}$ & $\begin{array}{l}\text { Some people } \\
\text { said it is good } \\
\text { and others } \\
\text { complained } \\
\text { of heat. }\end{array}$ & Feel good \\
\hline 25 & $\begin{array}{l}\text { How do you feel } \\
\text { about using bed } \\
\text { nets? }\end{array}$ & $\begin{array}{l}\text { Bear the heat } \\
\text { but avoids the } \\
\text { chemical } \\
\text { from touching } \\
\text { his skin }\end{array}$ & $\begin{array}{l}\text { Feel very } \\
\text { good } \\
\text { because } \\
\text { mosquito } \\
\text { won't come } \\
\text { around }\end{array}$ & $\begin{array}{l}\text { Feel very } \\
\text { fine. }\end{array}$ & Feel fine \\
\hline 26 & $\begin{array}{l}\text { What things do } \\
\text { you consider } \\
\text { health risks in your } \\
\text { tribe or culture? } \\
\text { (i.e. malaria, TB) }\end{array}$ & $\begin{array}{l}\text { Dumpsite, } \\
\text { dirty water, } \\
\text { open-latrines }\end{array}$ & $\begin{array}{l}\text { Dumpsite, } \\
\text { feces } \\
\text { wrapped and } \\
\text { thrown } \\
\text { around }\end{array}$ & dirtiness & dirt \\
\hline 27 & $\begin{array}{l}\text { How do you treat } \\
\text { health risks in your } \\
\text { tribe or culture? }\end{array}$ & Clean the area & $\begin{array}{l}\text { Clean the } \\
\text { environment }\end{array}$ & $\begin{array}{l}\text { Clean dirt } \\
\text { from around } \\
\text { house }\end{array}$ & \\
\hline 28 & $\begin{array}{l}\text { How do you } \\
\text { communicate } \\
\text { health risk in your } \\
\text { tribe or culture? }\end{array}$ & $\begin{array}{l}\text { Drum or } \\
\text { sankpa sound }\end{array}$ & Radio & $\begin{array}{l}\text { Radio or } \\
\text { drum }\end{array}$ & Word of mouth \\
\hline
\end{tabular}




\begin{tabular}{|c|c|c|c|c|c|}
\hline 29 & $\begin{array}{l}\text { Do you use radio, } \\
\text { television, flyer, or } \\
\text { drum to } \\
\text { communicate } \\
\text { health risks? }\end{array}$ & & & & \\
\hline 30 & $\begin{array}{l}\text { Explain some of } \\
\text { the difficulties in } \\
\text { communicating } \\
\text { health risks in your } \\
\text { tribe or culture? }\end{array}$ & & $\begin{array}{l}\text { Word of } \\
\text { mouth and } \\
\text { door to door }\end{array}$ & No problem & \\
\hline 31 & $\begin{array}{l}\text { How do the people } \\
\text { who distribute the } \\
\text { bed nets } \\
\text { communicate with } \\
\text { you? }\end{array}$ & $\begin{array}{l}\text { Talk good. } \\
\text { Can't sleep } \\
\text { without the } \\
\text { net and don't } \\
\text { sell it }\end{array}$ & $\begin{array}{l}\text { Over radio } \\
\text { and they } \\
\text { showed him } \\
\text { how to do it } \\
\text { clearly }\end{array}$ & $\begin{array}{l}\text { Word of } \\
\text { mouth }\end{array}$ & Word of mouth \\
\hline 32 & $\begin{array}{l}\text { Do you feel you } \\
\text { usually get clear } \\
\text { information on } \\
\text { malaria treatment } \\
\text { and health risks? }\end{array}$ & & & clear & \\
\hline 33 & $\begin{array}{l}\text { Whether you } \\
\text { answer yes or no, } \\
\text { how do you think } \\
\text { health risks should } \\
\text { be communicated? }\end{array}$ & & & & \\
\hline 34 & $\begin{array}{l}\text { Should your } \\
\text { language, culture, } \\
\text { or tradition matter } \\
\text { in communicating } \\
\text { health risks to } \\
\text { you? }\end{array}$ & $\begin{array}{l}\text { Understands } \\
\text { better when } \\
\text { explained in } \\
\text { the tribe }\end{array}$ & Language & yes & Yes language \\
\hline 35 & $\begin{array}{l}\text { If so, why and in } \\
\text { what ways? }\end{array}$ & & $\begin{array}{l}\text { Language is } \\
\text { most } \\
\text { important } \\
\text { because } \\
\text { majority of } \\
\text { my people } \\
\text { with } \\
\text { understand it } \\
\text { better }\end{array}$ & $\begin{array}{l}\text { Language is } \\
\text { important } \\
\text { because many } \\
\text { of our people } \\
\text { can't } \\
\text { understand } \\
\text { English so } \\
\text { they will } \\
\text { better listen to } \\
\text { us }\end{array}$ & No sure \\
\hline \multicolumn{6}{|c|}{36 If not, why not? } \\
\hline 37 & $\begin{array}{l}\text { Do you } \\
\text { recommend any } \\
\text { changes in the way } \\
\text { information about } \\
\text { bed nets is } \\
\text { communicated? }\end{array}$ & okay & $\begin{array}{l}\text { Not } \\
\text { sufficient }\end{array}$ & okay & okay \\
\hline
\end{tabular}




\begin{tabular}{|c|c|c|c|c|c|}
\hline \# & Question & $\begin{array}{c}\text { Participant } \\
\quad \# 21\end{array}$ & $\begin{array}{l}\text { Participant } \\
\quad \# 22\end{array}$ & $\begin{array}{c}\text { Participant } \\
\# 23\end{array}$ & $\begin{array}{c}\text { Participant } \\
\# 24\end{array}$ \\
\hline 1. & $\begin{array}{l}\text { Are you } 18 \text { or } \\
\text { older? }\end{array}$ & Older male & Older male & Older man & Older male \\
\hline 2. & $\begin{array}{l}\text { If so, are you } \\
\text { between ages } 18-29 \text {, } \\
30-49,50-59 \text {, or } 60 \\
\text { and over? }\end{array}$ & $18-29$ & $18-29$ & $50-59$ & $50-59$ \\
\hline 3. & $\begin{array}{l}\text { Are you traditional } \\
\text { leader/elder, mother, } \\
\text { young adult (male } \\
\text { or female), or head } \\
\text { of household? }\end{array}$ & $\begin{array}{l}\text { Young adult } \\
\text { male }\end{array}$ & Young adult & $\begin{array}{l}\text { Traditional } \\
\text { leader }\end{array}$ & hoh \\
\hline 4. & $\begin{array}{l}\text { Do you live in this } \\
\text { city, village, or } \\
\text { community? }\end{array}$ & $\begin{array}{l}\text { No, Vai } \\
\text { town }\end{array}$ & No, Vai town & $\begin{array}{l}\text { No. I live on } \\
\text { New York } \\
\text { street }\end{array}$ & $\begin{array}{l}\text { Yes, Kpelle } \\
\text { town }\end{array}$ \\
\hline 5. & $\begin{array}{l}\text { If yes, how long } \\
\text { have you lived in } \\
\text { this city, village, or } \\
\text { community? }\end{array}$ & 20 yrs. & 5 yrs. & 20 yrs. & 6 months \\
\hline 6. & $\begin{array}{l}\text { Which ethnic group } \\
\text { do you belong to? }\end{array}$ & Bassa & Bassa & Mandingo & Mandingo \\
\hline 7. & $\begin{array}{l}\text { Which language(s) } \\
\text { do you speak? }\end{array}$ & $\begin{array}{l}\text { Bassa and } \\
\text { English }\end{array}$ & $\begin{array}{l}\text { Bassa and } \\
\text { English }\end{array}$ & $\begin{array}{l}\text { Mandingo } \\
\text { and English }\end{array}$ & $\begin{array}{l}\text { Mandingo and } \\
\text { English }\end{array}$ \\
\hline 8. & $\begin{array}{l}\text { Which religion do } \\
\text { you belong to? }\end{array}$ & Christian & Christian & Muslim & Muslim \\
\hline 9. & $\begin{array}{l}\text { Have you ever heard } \\
\text { of malaria? }\end{array}$ & yes & yes & yes & yes \\
\hline 10. & $\begin{array}{l}\text { What is the name } \\
\text { for malaria in your } \\
\text { local language? }\end{array}$ & Sun-nee & Sun-nee & farlee & sumaya \\
\hline 11. & $\begin{array}{l}\text { Have you ever } \\
\text { suffered from } \\
\text { malaria? }\end{array}$ & yes & yes & yes & yes \\
\hline 12. & $\begin{array}{l}\text { If no, what would } \\
\text { you do if you got } \\
\text { malaria? }\end{array}$ & & & & \\
\hline 13. & $\begin{array}{l}\text { If yes, what kinds of } \\
\text { treatments do take } \\
\text { for malaria? }\end{array}$ & $\begin{array}{l}\text { Only } \\
\text { amodiaquine }\end{array}$ & $\begin{array}{l}\text { Only } \\
\text { amodiaquine }\end{array}$ & $\begin{array}{l}\text { Country } \\
\text { medicine and } \\
\text { it can cure } \\
\text { me. } \\
\text { Ganagana, } \\
\text { jologbo, and } \\
\text { pineapple } \\
\text { leave. } \\
\text { Put ganagana } \\
\text { in bottle drink } \\
\text { before eating. } \\
\text { Take western } \\
\text { treatment but } \\
\text { mostly } \\
\text { country } \\
\text { medicine }\end{array}$ & $\begin{array}{l}\text { Ganagana } \\
\text { (root) from a } \\
\text { three; carnee } \\
\text { (fruit). Still in } \\
\text { bottle for } 3 \\
\text { days and drink. } \\
\text { Buy drugs } \\
\text { from hospital }\end{array}$ \\
\hline
\end{tabular}




\begin{tabular}{|c|c|c|c|c|c|}
\hline 14. & $\begin{array}{l}\text { Do you use bed nets } \\
\text { to fight off malaria? }\end{array}$ & yes & yes & yes & no \\
\hline 15. & If not, why not? & & & & $\begin{array}{l}\text { Plan to get it } \\
\text { from ngo }\end{array}$ \\
\hline 16. & $\begin{array}{l}\text { What do you use to } \\
\text { fight off malaria? }\end{array}$ & & & & $\begin{array}{l}\text { Mosquito } \\
\text { chord after } 1 \text { to } \\
2 \text { hours }\end{array}$ \\
\hline 17. & Does it help? & & & & yes \\
\hline 18. & How do you know? & & & & $\begin{array}{l}\text { Mosquito will } \\
\text { die after two } \\
\text { hours and cut } \\
\text { off chord and } \\
\text { sleep }\end{array}$ \\
\hline 19. & $\begin{array}{l}\text { If yes, how did you } \\
\text { learn about bed nets } \\
\text { (i.e. radio, word of } \\
\text { mouth, health } \\
\text { clinic)? }\end{array}$ & radio & $\begin{array}{l}\text { Word of } \\
\text { mouth, radio, } \\
\text { health clinic }\end{array}$ & Radio, clinic & \\
\hline 20. & $\begin{array}{l}\text { Which malaria } \\
\text { treatment program } \\
\text { did you get your bed } \\
\text { nets from? }\end{array}$ & $\begin{array}{l}\text { Government } \\
\text { hospital }\end{array}$ & $\begin{array}{l}\text { Malaria } \\
\text { initiative }\end{array}$ & & \\
\hline 21. & $\begin{array}{l}\text { How long have you } \\
\text { used bed nets? }\end{array}$ & 6 yrs. & 2 & 3 yrs. & \\
\hline 22. & $\begin{array}{l}\text { Did someone teach } \\
\text { you how to use bed } \\
\text { nets? }\end{array}$ & $\begin{array}{l}\text { No; learn on } \\
\text { own }\end{array}$ & yes & & \\
\hline 23. & $\begin{array}{l}\text { How many people } \\
\text { in your household } \\
\text { use bed nets? }\end{array}$ & 8 & 2 & 8 & \\
\hline 24. & $\begin{array}{l}\text { How do people in } \\
\text { your tribe or culture } \\
\text { feel about using bed } \\
\text { nets? }\end{array}$ & $\begin{array}{l}\text { Fine. No } \\
\text { mosquito } \\
\text { with bednet }\end{array}$ & comfortable & $\begin{array}{l}\text { People feel } \\
\text { fine to use net } \\
\text { because it } \\
\text { protects them } \\
\text { from malaria } \\
\end{array}$ & $\begin{array}{l}\text { Feel happy } \\
\text { about bed net } \\
\text { because it stops } \\
\text { mosquito; ngo } \\
\text { spray rooms } \\
\end{array}$ \\
\hline 25. & $\begin{array}{l}\text { How do you feel } \\
\text { about using bed } \\
\text { nets? }\end{array}$ & fine & comfortable & $\begin{array}{l}\text { Very good. } \\
\text { Ask his } \\
\text { people to use } \\
\text { it because it is } \\
\text { good }\end{array}$ & Feel good \\
\hline 26. & $\begin{array}{l}\text { What things do you } \\
\text { consider health risks } \\
\text { in your tribe or } \\
\text { culture? (i.e. } \\
\text { malaria, TB) }\end{array}$ & & $\begin{array}{l}\text { Anything that } \\
\text { affects the } \\
\text { health }\end{array}$ & Pupu and flies & $\begin{array}{l}\text { Pollution from } \\
\text { dumpsite too } \\
\text { close to living } \\
\text { premises brigs } \\
\text { flies and } \\
\text { mosquitos } \\
\end{array}$ \\
\hline 27. & $\begin{array}{l}\text { How do you treat } \\
\text { health risks in your } \\
\text { tribe or culture? }\end{array}$ & & $\begin{array}{l}\text { Cleaning } \\
\text { around house } \\
\text { and the } \\
\text { environment }\end{array}$ & $\begin{array}{l}\text { Ask people to } \\
\text { clean their } \\
\text { environment }\end{array}$ & \\
\hline 28. & $\begin{array}{l}\text { How do you } \\
\text { communicate health } \\
\text { risk in your tribe or } \\
\text { culture? }\end{array}$ & $\begin{array}{l}\text { Word of } \\
\text { mouth }\end{array}$ & Word of mouth & $\begin{array}{l}\text { Word of } \\
\text { mouth }\end{array}$ & $\begin{array}{l}\text { Twice in the } \\
\text { month hold } \\
\text { meeting to } \\
\text { clean } \\
\text { surrounding of } \\
\text { house and yard }\end{array}$ \\
\hline
\end{tabular}




\begin{tabular}{|c|c|c|c|c|c|}
\hline 29. & $\begin{array}{l}\text { Do you use radio, } \\
\text { television, flyer, or } \\
\text { drum to } \\
\text { communicate health } \\
\text { risks? }\end{array}$ & & & & \\
\hline 30. & $\begin{array}{l}\text { Explain some of the } \\
\text { difficulties in } \\
\text { communicating } \\
\text { health risks in your } \\
\text { tribe or culture? }\end{array}$ & no & $\begin{array}{l}\text { Not acquainted } \\
\text { with his own } \\
\text { dialect }\end{array}$ & & \\
\hline 31. & $\begin{array}{l}\text { How do the people } \\
\text { who distribute the } \\
\text { bed nets } \\
\text { communicate with } \\
\text { you? }\end{array}$ & $\begin{array}{l}\text { Through } \\
\text { radio }\end{array}$ & $\begin{array}{l}\text { Verbally and } \\
\text { vernacular } \\
\text { languages }\end{array}$ & & \\
\hline 32. & $\begin{array}{l}\text { Do you feel you } \\
\text { usually get clear } \\
\text { information on } \\
\text { malaria treatment } \\
\text { and health risks? }\end{array}$ & yes & & & \\
\hline 33. & $\begin{array}{l}\text { Whether you answer } \\
\text { yes or no, how do } \\
\text { you think health } \\
\text { risks should be } \\
\text { communicated? }\end{array}$ & & & & \\
\hline 34. & $\begin{array}{l}\text { Should your } \\
\text { language, culture, or } \\
\text { tradition matter in } \\
\text { communicating } \\
\text { health risks to you? }\end{array}$ & yes & yes & yes & yes \\
\hline 35. & $\begin{array}{l}\text { If so, why and in } \\
\text { what ways? }\end{array}$ & $\begin{array}{l}\text { Important } \\
\text { because the } \\
\text { environment } \\
\text { he lives in }\end{array}$ & $\begin{array}{l}\text { More people } \\
\text { not educated } \\
\text { and people will } \\
\text { relate well in } \\
\text { their language }\end{array}$ & $\begin{array}{l}\text { Important } \\
\text { because when } \\
\text { I speak my } \\
\text { own language } \\
\text { my people } \\
\text { can } \\
\text { understand } \\
\text { me very good. }\end{array}$ & $\begin{array}{l}\text { Language is } \\
\text { important for } \\
\text { understanding }\end{array}$ \\
\hline 36. & If not, why not? & & & & \\
\hline 37. & $\begin{array}{l}\text { Do you recommend } \\
\text { any changes in the } \\
\text { way information } \\
\text { about bed nets is } \\
\text { communicated? }\end{array}$ & $\begin{array}{l}\text { No } \\
\text { comments }\end{array}$ & $\begin{array}{l}\text { Use the media } \\
\text { and move from } \\
\text { urban areas to } \\
\text { rural areas and } \\
\text { communicate } \\
\text { in rural } \\
\text { languages }\end{array}$ & okay & $\begin{array}{l}\text { Yes. Because } \\
\text { bed net that } \\
\text { some chemical } \\
\text { in it but it can't } \\
\text { be washed. We } \\
\text { used to wash } \\
\text { bed nets before } \\
\text { war; so } \\
\text { somehow there } \\
\text { is some } \\
\text { problem }\end{array}$ \\
\hline
\end{tabular}




\begin{tabular}{|c|c|c|c|c|c|}
\hline$\#$ & Question & $\begin{array}{c}\text { Participant } \\
\text { \#25 }\end{array}$ & $\begin{array}{c}\text { Participant } \\
\text { \#26 }\end{array}$ & $\begin{array}{c}\text { Participant } \\
\text { \#27 }\end{array}$ & $\begin{array}{l}\text { Participant } \\
\text { \#28 }\end{array}$ \\
\hline 1. & $\begin{array}{l}\text { Are you } 18 \text { or } \\
\text { older? }\end{array}$ & Yes male & Older male & Older male & Older male \\
\hline 2. & $\begin{array}{l}\text { If so, are you } \\
\text { between ages } 18-29 \text {, } \\
30-49,50-59 \text {, or } 60 \\
\text { and over? }\end{array}$ & $30-49$ & $30-49$ & $30-49$ & $30-49$ \\
\hline 3. & $\begin{array}{l}\text { Are you traditional } \\
\text { leader/elder, } \\
\text { mother, young adult } \\
\text { (male or female), or } \\
\text { head of household? }\end{array}$ & hoh & hoh & hoh & $\begin{array}{l}\text { Traditional } \\
\text { leader }\end{array}$ \\
\hline 4. & $\begin{array}{l}\text { Do you live in this } \\
\text { city, village, or } \\
\text { community? }\end{array}$ & $\begin{array}{l}\text { No, } \\
\text { Compound } \\
\text { community }\end{array}$ & $\begin{array}{l}\text { No, } \\
\text { Fairground }\end{array}$ & $\begin{array}{l}\text { No, } \\
\text { Compound } \\
\text { community }\end{array}$ & $\begin{array}{c}\text { No. I live in } \\
\text { Kingsville } \\
\text { community, } \\
\text { district \#1 }\end{array}$ \\
\hline 5. & $\begin{array}{l}\text { If yes, how long } \\
\text { have you lived in } \\
\text { this city, village, or } \\
\text { community? }\end{array}$ & $1 \mathrm{yr}$. & 15 yrs. & Since birth & 22 yrs. \\
\hline 6. & $\begin{array}{l}\text { Which ethnic group } \\
\text { do you belong to? }\end{array}$ & Mandingo & Mandingo & Bassa & Bassa \\
\hline 7. & $\begin{array}{l}\text { Which language(s) } \\
\text { do you speak? }\end{array}$ & $\begin{array}{l}\text { Mandingo } \\
\text { and English }\end{array}$ & $\begin{array}{l}\text { Mandingo } \\
\text { and English }\end{array}$ & $\begin{array}{l}\text { Bassa and } \\
\text { English }\end{array}$ & $\begin{array}{l}\text { Bassa and } \\
\text { English }\end{array}$ \\
\hline 8. & $\begin{array}{l}\text { Which religion do } \\
\text { you belong to? }\end{array}$ & Muslim & Muslim & Christian & Christian \\
\hline 9. & $\begin{array}{l}\text { Have you ever } \\
\text { heard of malaria? }\end{array}$ & yes & yes & yes & yes \\
\hline 10. & $\begin{array}{l}\text { What is the name } \\
\text { for malaria in your } \\
\text { local language? }\end{array}$ & farlee & Nen-nen & Sun-nee & Sun-nee \\
\hline 11. & $\begin{array}{l}\text { Have you ever } \\
\text { suffered from } \\
\text { malaria? }\end{array}$ & yes & yes & yes & yes \\
\hline 12. & $\begin{array}{l}\text { If no, what would } \\
\text { you do if you got } \\
\text { malaria? }\end{array}$ & & & & \\
\hline
\end{tabular}




\begin{tabular}{|c|c|c|c|c|c|}
\hline 13. & $\begin{array}{l}\text { If yes, what } \\
\text { kinds of } \\
\text { treatments } \\
\text { do take for } \\
\text { malaria? }\end{array}$ & $\begin{array}{l}\text { Hospital and } \\
\text { country } \\
\text { medicine Bitter } \\
\text { leave called } \\
\text { casi-ah; boiled } \\
\text { it and take bath } \\
\text { with it with } \\
\text { black soap. } \\
\text { Kills malaria } 2 \\
\text { to } 4 \text { times you } \\
\text { get cure }\end{array}$ & $\begin{array}{l}\text { Hospital } \\
\text { and country } \\
\text { medicine. } \\
\text { Sekou } \\
\text { toure leave, } \\
\text { ganagana, } \\
\text { copa }\end{array}$ & $\begin{array}{l}\text { Well, we take } \\
\text { some medical } \\
\text { treatment. It is } \\
\text { not traditional but } \\
\text { we. Go to the } \\
\text { hospital and take } \\
\text { treatment. } \\
\text { Amodiaquine. } \\
\text { They call the } \\
\text { tablet } \\
\text { amodiaquine, but } \\
\text { when you take it } \\
\text { just like you } \\
\text { going to die. It is } \\
\text { not an easy tablet. } \\
\text { People at first } \\
\text { people used to } \\
\text { take } 4,5,6,7 \text {, } \\
\text { tablets per day. } \\
\text { This time we } \\
\text { reduced it to 2-2. } \\
\text { And it is not } \\
\text { easy. When you } \\
\text { take that malaria } \\
\text { drugs just like } \\
\text { you're going to } \\
\text { die. It is strong. } \\
\text { Well, that is the } \\
\text { only one that can } \\
\text { use me. } \\
\text { Sometimes I take } \\
\text { chlroquinne, but } \\
\text { if I use the } \\
\text { chlroquinne too } \\
\text { my ears and } \\
\text { everything can } \\
\text { be, yes. Well it } \\
\text { take long time } \\
\text { before I can hear } \\
\text { correctly again } \\
\end{array}$ & $\begin{array}{l}\text { Mostly and } \\
\text { presently country } \\
\text { medicine because } \\
\text { the drug } \\
\text { amodiaquine } \\
\text { when I take it my } \\
\text { whole ear can } \\
\text { lock. So I don't } \\
\text { take it. I usually } \\
\text { Take jologbo. It } \\
\text { is bitter; it takes } \\
\text { the malaria out of } \\
\text { you }\end{array}$ \\
\hline 14. & $\begin{array}{l}\text { Do you use } \\
\text { bed nets to } \\
\text { fight off } \\
\text { malaria? }\end{array}$ & yes & yes & $\begin{array}{l}\text { Well, I have bed } \\
\text { net but I don't } \\
\text { actually use it } \\
\text { oh. }\end{array}$ & no \\
\hline
\end{tabular}




\begin{tabular}{|c|c|c|c|c|c|}
\hline 15 . & $\begin{array}{l}\text { If not, why } \\
\text { not? }\end{array}$ & & & $\begin{array}{l}\text { Well, the heat, the } \\
\text { situation we find } \\
\text { ourselves in in } \\
\text { Liberia, the heat is } \\
\text { not easy so if } \\
\text { you're sleeping } \\
\text { under it. And firstly, } \\
\text { in the ... I watched } \\
\text { an African show } \\
\text { where people, the } \\
\text { people used to } \\
\text { really take care of } \\
\text { the net before } \\
\text { giving to people. } \\
\text { Like the net too has } \\
\text { some chemicals } \\
\text { within, so they can } \\
\text { wash it, dry it out, } \\
\text { and give it out to } \\
\text { people. But they } \\
\text { just give it to you } \\
\text { and you don't really } \\
\text { know the way of } \\
\text { preparation; so } \\
\text { sometimes we have } \\
\text { fears too that when } \\
\text { you sleep under the } \\
\text { chemical it can } \\
\text { bring some affection } \\
\text { or something that } \\
\text { can happen to you. } \\
\text { The heat and for } \\
\text { that, for me } \\
\text { personally, I don't } \\
\text { sleep under the nets }\end{array}$ & $\begin{array}{l}\text { They } \\
\text { distributed } \\
\text { only two towns } \\
\text { and his town } \\
\text { was not } \\
\text { included }\end{array}$ \\
\hline 16. & $\begin{array}{l}\text { What do } \\
\text { you use to } \\
\text { fight off } \\
\text { malaria? }\end{array}$ & & & & $\begin{array}{l}\text { Burnt palm } \\
\text { kernel, put it in } \\
\text { pan an burn it } \\
\text { and the smoke } \\
\text { can drive the } \\
\text { mosquito out }\end{array}$ \\
\hline 17. & $\begin{array}{l}\text { Does it } \\
\text { help? }\end{array}$ & & & & yes \\
\hline 18. & $\begin{array}{l}\text { How do } \\
\text { you know? }\end{array}$ & & & & $\begin{array}{l}\text { The smoke } \\
\text { drives the } \\
\text { mosquito away }\end{array}$ \\
\hline 19. & $\begin{array}{l}\text { If yes, how } \\
\text { did you } \\
\text { learn about } \\
\text { bed nets } \\
\text { (i.e. radio, } \\
\text { word of } \\
\text { mouth, } \\
\text { health } \\
\text { clinic)? }\end{array}$ & $\begin{array}{l}\text { Radio and } \\
\text { clinic }\end{array}$ & $\begin{array}{l}\text { Radio and } \\
\text { health clinic }\end{array}$ & $\begin{array}{l}\text { Media and health } \\
\text { workers }\end{array}$ & \\
\hline
\end{tabular}




\begin{tabular}{|c|c|c|c|c|c|}
\hline 20. & $\begin{array}{l}\text { Which } \\
\text { malaria } \\
\text { treatment } \\
\text { program } \\
\text { did you get } \\
\text { your bed } \\
\text { nets from? }\end{array}$ & merlin & merlin & Clinton foundation & \\
\hline 21. & $\begin{array}{l}\text { How long } \\
\text { have you } \\
\text { used bed } \\
\text { nets? }\end{array}$ & 5 yrs. & 3 yrs. & & \\
\hline 22. & $\begin{array}{l}\text { Did } \\
\text { someone } \\
\text { teach you } \\
\text { how to use } \\
\text { bed nets? }\end{array}$ & yes & yes & no & \\
\hline 23. & $\begin{array}{l}\text { How many } \\
\text { people in } \\
\text { your } \\
\text { household } \\
\text { use bed } \\
\text { nets? } \\
\end{array}$ & 5 & 8 & Besides my room, no & none \\
\hline 24. & $\begin{array}{l}\text { How do } \\
\text { people in } \\
\text { your tribe } \\
\text { or culture } \\
\text { feel about } \\
\text { using bed } \\
\text { nets? }\end{array}$ & $\begin{array}{l}\text { Fine because } \\
\text { it can protect } \\
\text { people from } \\
\text { malaria }\end{array}$ & $\begin{array}{l}\text { Feel } \\
\text { fine } \\
\text { because } \\
\text { it helps } \\
\text { to get } \\
\text { malaria } \\
\text { away }\end{array}$ & $\begin{array}{l}\text { Well, sometimes they feel } \\
\text { different because it is not } \\
\text { their culture, it is not their } \\
\text { way of doing things. They } \\
\text { are not actually used to it. } \\
\text { So aint they don't have the } \\
\text { time to use that; maybe we } \\
\text { that are to the citiyside, we } \\
\text { try but other people in our } \\
\text { rural areas don't have time } \\
\text { for that. Yes, in the sense } \\
\text { that using people feel that } \\
\text { using the net is just a waste } \\
\text { of time because they don't } \\
\text { have the facilities. Ain, } \\
\text { Somebody is almost } \\
\text { sleeping almost on the } \\
\text { ground and then you have } \\
\text { the net hanging. They will } \\
\text { feel like they have been } \\
\text { jailed, and something } \\
\text { surrounding them that they } \\
\text { are not used to. Also most } \\
\text { of them complained on the } \\
\text { heat. Sometimes the net } \\
\text { when it touches your face, } \\
\text { it itch it; it touch certain } \\
\text { parts of your body, it itch } \\
\text { it, so they have a lot of fear } \\
\text { of using the the mosquito } \\
\text { net. }\end{array}$ & $\begin{array}{l}\text { They will } \\
\text { feel fine if } \\
\text { they got it. If } \\
\text { they have it } \\
\text { to use it will } \\
\text { be alright for } \\
\mathrm{r} \text { them }\end{array}$ \\
\hline
\end{tabular}




\begin{tabular}{|c|c|c|c|c|c|}
\hline 25. & $\begin{array}{l}\text { How do } \\
\text { you feel } \\
\text { about using } \\
\text { bed nets? }\end{array}$ & Feel fine & $\begin{array}{l}\text { Feel } \\
\text { very } \\
\text { very } \\
\text { fine }\end{array}$ & $\begin{array}{l}\text { Well, actually, I don't } \\
\text { really feel fine because it is } \\
\text { not comfortable for me. It's } \\
\text { that People say you use the } \\
\text { net to protect the mosquito. } \\
\text { But sometimes I strongly } \\
\text { feel like it doesn't. Because } \\
\text { they give you; I have a } \\
\text { family bed and they give } \\
\text { me a single-bed mosquito } \\
\text { net and it can't cover my } \\
\text { bed. And Mosquito will go } \\
\text { all through there and it is } \\
\text { just a matter of waste of } \\
\text { time. And the heat again, } \\
\text { so I don't feel comfortable } \\
\text { using that. }\end{array}$ & $\begin{array}{l}\text { He will feel } \\
\text { comfortable } \\
\text { if he had it. }\end{array}$ \\
\hline 26. & $\begin{array}{l}\text { What } \\
\text { things do } \\
\text { you } \\
\text { consider } \\
\text { health risks } \\
\text { in your } \\
\text { tribe or } \\
\text { culture? } \\
\text { (i.e. } \\
\text { malaria, } \\
\text { TB) }\end{array}$ & $\begin{array}{l}\text { Dumpsite in } \\
\text { community } \\
\text { not correct } \\
\text { and hole } \\
\text { brings } \\
\text { mosquito to } \\
\text { lay egg with } \\
\text { water in hole }\end{array}$ & & $\begin{array}{l}\text { Ah, actually, what we really } \\
\text { consider health risk is the } \\
\text { issue of dumpsite, dirty } \\
\text { water, and they have been } \\
\text { taught to keep all those } \\
\text { things away from } \\
\text { themselves. }\end{array}$ & $\begin{array}{l}\text { Mosquitos } \\
\text { (when- } \\
\text { when) and } \\
\text { cow-fly can } \\
\text { cause river } \\
\text { blindness } \\
\text { because they } \\
\text { live near the } \\
\text { river }\end{array}$ \\
\hline 27. & $\begin{array}{l}\text { How do } \\
\text { you treat } \\
\text { health risks } \\
\text { in your } \\
\text { tribe or } \\
\text { culture? }\end{array}$ & & & $\begin{array}{l}\text { Keep dumpsite and other } \\
\text { things from your area. }\end{array}$ & $\begin{array}{l}\text { Cover those } \\
\text { holes with } \\
\text { water in } \\
\text { them, except } \\
\text { for the river } \\
\text { bank }\end{array}$ \\
\hline 28. & $\begin{array}{l}\text { How do } \\
\text { you } \\
\text { communica } \\
\text { te health } \\
\text { risk in your } \\
\text { tribe or } \\
\text { culture? }\end{array}$ & $\begin{array}{l}\text { Word of } \\
\text { mouth }\end{array}$ & & $\begin{array}{l}\text { What is not actually } \\
\text { mentioned is this face-to- } \\
\text { face talk when we have } \\
\text { some groups that go into } \\
\text { the community speaking } \\
\text { the local dialects to explain. } \\
\text { Sometimes people use the } \\
\text { media to do some } \\
\text { promotional something. }\end{array}$ & $\begin{array}{l}\text { By palava- } \\
\text { hut and } \\
\text { townhall } \\
\text { meeting }\end{array}$ \\
\hline 29. & $\begin{array}{l}\text { Do you use } \\
\text { radio, } \\
\text { television, } \\
\text { flyer, or } \\
\text { drum to } \\
\text { communica } \\
\text { te health } \\
\text { risks? }\end{array}$ & radio & & & \\
\hline
\end{tabular}




\begin{tabular}{|c|c|c|c|c|c|}
\hline 30. & $\begin{array}{l}\text { Explain some of } \\
\text { the difficulties } \\
\text { in } \\
\text { communicating } \\
\text { health risks in } \\
\text { your tribe or } \\
\text { culture? }\end{array}$ & & & $\begin{array}{l}\text { Outside the city, once the people } \\
\text { are farming, it can be very } \\
\text { difficult to get them together to } \\
\text { spread the message; especially if } \\
\text { you talk about mosquito; they will } \\
\text { complain that anything can kill } \\
\text { you, so that mosquito business I } \\
\text { will leave my farm and sit here? } \\
\text { When the people are farming, it } \\
\text { looks difficult to get them } \\
\text { together at times }\end{array}$ & $\begin{array}{l}\text { Yes. Even if } \\
\text { the } \\
\text { towncrier } \\
\text { needs to be } \\
\text { paid; if not } \\
\text { they will not } \\
\text { relay the } \\
\text { message }\end{array}$ \\
\hline 31. & $\begin{array}{l}\text { How do the } \\
\text { people who } \\
\text { distribute the } \\
\text { bed nets } \\
\text { communicate } \\
\text { with you? } \\
\end{array}$ & & & & \\
\hline 32. & $\begin{array}{l}\text { Do you feel you } \\
\text { usually get clear } \\
\text { information on } \\
\text { malaria } \\
\text { treatment and } \\
\text { health risks? } \\
\end{array}$ & & & & \\
\hline 33. & $\begin{array}{l}\text { Whether you } \\
\text { answer yes or } \\
\text { no, how do you } \\
\text { think health } \\
\text { risks should be } \\
\text { communicated? }\end{array}$ & & & & \\
\hline 34. & $\begin{array}{l}\text { Should your } \\
\text { language, } \\
\text { culture, or } \\
\text { tradition matter } \\
\text { in } \\
\text { communicating } \\
\text { health risks to } \\
\text { you? }\end{array}$ & yes & yes & $\begin{array}{l}\text { Seriously it matters because that } \\
\text { is what our people understand. } \\
\text { You can't go anywhere and speak } \\
\text { big English; you got to speak our } \\
\text { own dialects to them telling them } \\
\text { the risks. }\end{array}$ & $\begin{array}{l}\text { Exactly. } \\
\text { yes, very } \\
\text { important } \\
\text { and they } \\
\text { mobilize in } \\
\text { my message } \\
\text { they will } \\
\text { understand it } \\
\text { better }\end{array}$ \\
\hline
\end{tabular}




\begin{tabular}{|c|c|c|c|c|}
\hline 35. & $\begin{array}{l}\text { If so, why } \\
\text { and in } \\
\text { what } \\
\text { ways? }\end{array}$ & $\begin{array}{l}\text { When } \\
\text { we } \\
\text { speak } \\
\text { tribe it } \\
\text { is } \\
\text { simple } \\
\text { to } \\
\text { underst } \\
\text { and but } \\
\text { difficult } \\
\text { in } \\
\text { English }\end{array}$ & $\begin{array}{l}\text { Speaki } \\
\text { ng in } \\
\text { langua } \\
\text { ge is } \\
\text { better } \\
\text { becaus } \\
\text { e } \\
\text { many } \\
\text { people } \\
\text { didn't } \\
\text { go to } \\
\text { school } \\
\text { and } \\
\text { don't } \\
\text { speak } \\
\text { Englis } \\
\text { h well. }\end{array}$ & $\begin{array}{l}\text { Because why because it is the } \\
\text { greatest thing our people } \\
\text { understand. Even I who here } \\
\text { where I say I try to learn one two } \\
\text { book, graduated from high school } \\
\text { and going to college, there are } \\
\text { certain, certain things if you } \\
\text { communicate with me, I still want } \\
\text { to understand it in my dialect. So } \\
\text { it is very very important to our } \\
\text { people that you use your own } \\
\text { local dialect to them so that they } \\
\text { can be able to understand it } \\
\text { clearly. I don't care how you } \\
\text { speak the lowest English, the } \\
\text { Liberian English, to them, there is } \\
\text { a need, a need to what, speak that } \\
\text { dialect. And you can do that } \\
\text { through the means of radio, } \\
\text { through the means of towncrier, } \\
\text { because sometimes it is good to } \\
\text { use our people um because they } \\
\text { often listen to them. Towncrier } \\
\text { going around and telling the } \\
\text { people, and educating them and } \\
\text { they listen more. }\end{array}$ \\
\hline 36. & $\begin{array}{l}\text { If not, } \\
\text { why not? }\end{array}$ & & & \\
\hline
\end{tabular}




\begin{tabular}{|c|c|c|c|c|c|}
\hline 37. & $\begin{array}{l}\text { Do you } \\
\text { recommen } \\
d \text { any } \\
\text { changes in } \\
\text { the way } \\
\text { informatio } \\
n \text { about } \\
\text { bed nets is } \\
\text { communic } \\
\text { ated? }\end{array}$ & okay & $\begin{array}{l}\text { Okay } \\
\text { alrig } \\
\text { ht } \\
\text { right } \\
\text { now }\end{array}$ & $\begin{array}{l}\text { Yes, my own recommendation to our } \\
\text { donors or whosoever that is doing this. I } \\
\text { want to recommend to them strong that } \\
\text { indeed the issue of the net, the education } \\
\text { should be more. But sometimes because } \\
\text { of finance, or whatsoever, they think they } \\
\text { just get over the media without getting to } \\
\text { the local people. So what happening there } \\
\text { is not everybody in our own locale having } \\
\text { radio--can listen to radio---so while there's } \\
\text { the need that--air people go in the } \\
\text { community--once you want to help the } \\
\text { person, if your meet face-to-face, from } \\
\text { your own interaction, telling them the } \\
\text { danger in their own local dialect, then I } \\
\text { strongly feel that they will take that into } \\
\text { consideration. But because hearing it over } \\
\text { media and it is not benefiting them --like } \\
\text { for food you say you eating, and some } \\
\text { people are not listening so when they } \\
\text { collect the net they put it under their cover } \\
\text { and then go about their business because } \\
\text { the education is not there. So there's a } \\
\text { need that our people go into the } \\
\text { community to talk with our people. It is } \\
\text { not only Bassa people living in Grand } \\
\text { Bassa, there are other Kpelle people--like } \\
\text { this is called Kpelle area; they can go } \\
\text { there, the Kpelle people speak Kpelle to } \\
\text { the people, telling them the danger of not } \\
\text { beople, the house-to-house so that they can } \\
\text { using the net } \\
\text { so. But then, and then, let them be able to } \\
\text { prepare the net; they should not bring it } \\
\text { raw--there are some effects that it has on -- } \\
\text { that net has on us. Because for one fact, } \\
\text { the chemical in that net we don't know } \\
\text { what kind of effect it has. So even my } \\
\text { very self, if that net you don't put it in the } \\
\text { sun for certain time, it touch you. It itch } \\
\text { you the whole day. So those things should } \\
\text { be prepared before giving out to our } \\
\text { people because sometimes if the malaria } \\
\text { na kill you that the net will kill you. } \\
\text { Because our people don't really, look at } \\
\text { it is not actually prepared. And education } \\
\text { is one matter that people need to do. And } \\
\text { the media,, not only drummers }\end{array}$ & $\begin{array}{l}\text { Yes. The } \\
\text { chemical } \\
\text { is very } \\
\text { strong. I } \\
\text { once } \\
\text { visited my } \\
\text { aunt and } \\
\text { went } \\
\text { under the } \\
\text { net my } \\
\text { whole } \\
\text { face was } \\
\text { burning. } \\
\text { the } \\
\text { chemical } \\
\text { was too } \\
\text { wrong. } \\
\text { Burned } \\
\text { his face } \\
\text { and he } \\
\text { had to rub } \\
\text { red palm } \\
\text { oil }\end{array}$ \\
\hline
\end{tabular}




\begin{tabular}{|c|c|c|c|c|c|}
\hline$\#$ & Question & $\begin{array}{c}\text { Participant } \\
\# 29\end{array}$ & $\begin{array}{c}\text { Participant } \\
\text { \#30 }\end{array}$ & $\begin{array}{c}\text { Participant } \\
\# 31\end{array}$ & Participant \#32 \\
\hline 1. & Are you 18 or older? & Older male & Older male & Older male & Older female \\
\hline 2. & $\begin{array}{l}\text { If so, are you } \\
\text { between ages } 18-29 \text {, } \\
30-49,50-59 \text {, or } 60 \\
\text { and over? }\end{array}$ & $30-49$ & $\begin{array}{l}60 \text { and over } \\
\text { (74) }\end{array}$ & $50-59$ & $30-49$ \\
\hline 3. & $\begin{array}{l}\text { Are you traditional } \\
\text { leader/elder, mother, } \\
\text { young adult (male or } \\
\text { female), or head of } \\
\text { household? }\end{array}$ & $\begin{array}{l}\text { Young adult } \\
\text { male }\end{array}$ & $\begin{array}{l}\text { Traditional } \\
\text { leader }\end{array}$ & elder & $\begin{array}{l}\text { Mother, and } \\
\text { traditional } \\
\text { leader }\end{array}$ \\
\hline 4. & $\begin{array}{l}\text { Do you live in this } \\
\text { city, village, or } \\
\text { community? }\end{array}$ & $\begin{array}{l}\text { Sawmill } \\
\text { community }\end{array}$ & $\begin{array}{l}\text { Coone, } \\
\text { sanwein }\end{array}$ & sanwein & sanwein \\
\hline 5. & $\begin{array}{l}\text { If yes, how long } \\
\text { have you lived in } \\
\text { this city, village, or } \\
\text { community? }\end{array}$ & 20 yrs. & Born here & 3 yrs. & Born here \\
\hline 6. & $\begin{array}{l}\text { Which ethnic group } \\
\text { do you belong to? }\end{array}$ & Bassa & Bassa & Bassa & Kru \\
\hline 7. & $\begin{array}{l}\text { Which language(s) } \\
\text { do you speak? }\end{array}$ & $\begin{array}{l}\text { Bassa and } \\
\text { English }\end{array}$ & $\begin{array}{l}\text { Bassa and } \\
\text { Kru }\end{array}$ & $\begin{array}{l}\text { Bassa and } \\
\text { English }\end{array}$ & Bassa \\
\hline 8. & $\begin{array}{l}\text { Which religion do } \\
\text { you belong to? }\end{array}$ & Christian & Christian & Christian & Christian \\
\hline 9. & $\begin{array}{l}\text { Have you ever heard } \\
\text { of malaria? }\end{array}$ & $\begin{array}{l}\text { Yes more } \\
\text { than a million } \\
\text { times }\end{array}$ & yes & $\begin{array}{l}\text { Yes, I have } \\
\text { malaria } \\
\text { right now }\end{array}$ & yes \\
\hline 10 . & $\begin{array}{l}\text { What is the name for } \\
\text { malaria in your local } \\
\text { language? }\end{array}$ & Sun-nee & Sun-nee & $\begin{array}{l}\text { Sun-nee; } \\
\text { used to be } \\
\text { sunglie. } \\
\end{array}$ & $\begin{array}{l}\text { Sun-nee-sun- } \\
\text { glei }\end{array}$ \\
\hline 11. & $\begin{array}{l}\text { Have you ever } \\
\text { suffered from } \\
\text { malaria? }\end{array}$ & no & & yes & yes \\
\hline 12 . & $\begin{array}{l}\text { If no, what would } \\
\text { you do if you got } \\
\text { malaria? }\end{array}$ & $\begin{array}{l}\text { Go to hospital } \\
\text { so his case } \\
\text { can be } \\
\text { diagnosed for } \\
\text { rightful } \\
\text { treatment }\end{array}$ & & & \\
\hline 13. & $\begin{array}{l}\text { If yes, what kinds of } \\
\text { treatments do take } \\
\text { for malaria? }\end{array}$ & & $\begin{array}{l}\text { Traditional } \\
\text { medicine. } \\
\text { Swamp } \\
\text { grass, cut } \\
\text { the stems, } \\
\text { clean it, } \\
\text { boil and } \\
\text { drink. Take } \\
\text { tablets also }\end{array}$ & $\begin{array}{l}\text { Traditional } \\
\text { medicine. } \\
\text { Gold-color } \\
\text { suough- } \\
\text { jugg; } \\
\text { sometimes } \\
\text { it takes up } \\
\text { to } 2 \text { years } \\
\text { before } \\
\text { malaria } \\
\text { comes } \\
\text { again. Yes, } \\
\text { he takes } \\
\text { western } \\
\text { medicine } \\
\text { while in the } \\
\text { city }\end{array}$ & $\begin{array}{l}\text { Traditional. He } \\
\text { has medicine for } \\
\text { typhoid; three } \\
\text { types of } \\
\text { malaria. Butter } \\
\text { pearl leave, } \\
\text { Christmas bush, } \\
\text { etc. for typhoid, } \\
\text { the golden plum } \\
\text { leave, boil it and } \\
\text { drink. No, she } \\
\text { doesn't take } \\
\text { western } \\
\text { medicine. }\end{array}$ \\
\hline
\end{tabular}




\begin{tabular}{|c|c|c|c|c|c|}
\hline 14 . & $\begin{array}{l}\text { Do you use bed nets } \\
\text { to fight off malaria? }\end{array}$ & no & yes & yes & $\begin{array}{l}\text { No, although } \\
\text { she has three }\end{array}$ \\
\hline 15 . & If not, why not? & $\begin{array}{l}\text { Do not suffer } \\
\text { from malaria }\end{array}$ & & & \\
\hline 16. & $\begin{array}{l}\text { What do you use to } \\
\text { fight off malaria? }\end{array}$ & & & & \\
\hline 17. & Does it help? & & & & \\
\hline 18. & How do you know? & & & & \\
\hline 19. & $\begin{array}{l}\text { If yes, how did you } \\
\text { learn about bed nets } \\
\text { (i.e. radio, word of } \\
\text { mouth, health } \\
\text { clinic)? }\end{array}$ & & radio & $\begin{array}{l}\text { Word of } \\
\text { mouth; the } \\
\text { ngo brings } \\
\text { it and hangs } \\
\text { it up }\end{array}$ & \\
\hline 20. & $\begin{array}{l}\text { Which malaria } \\
\text { treatment program } \\
\text { did you get your bed } \\
\text { nets from? }\end{array}$ & & $\begin{array}{l}\text { Ngo (not } \\
\text { sure) }\end{array}$ & ngo & $\begin{array}{l}\text { Ngo; my } \\
\text { children put } \\
\text { theirs up but I } \\
\text { don't }\end{array}$ \\
\hline 21. & $\begin{array}{l}\text { How long have you } \\
\text { used bed nets? }\end{array}$ & & & 2 yrs. & \\
\hline 22. & $\begin{array}{l}\text { Did someone teach } \\
\text { you how to use bed } \\
\text { nets? }\end{array}$ & & yes & yes & \\
\hline 23. & $\begin{array}{l}\text { How many people in } \\
\text { your household use } \\
\text { bed nets? }\end{array}$ & & 10 persons & 3 & $\begin{array}{c}\text { 2; mosquito } \\
\text { doesn't bite me. }\end{array}$ \\
\hline 24. & $\begin{array}{l}\text { How do people in } \\
\text { your tribe or culture } \\
\text { feel about using bed } \\
\text { nets? }\end{array}$ & $\begin{array}{l}\text { I have not } \\
\text { actually taken } \\
\text { a survey, but I } \\
\text { think people } \\
\text { are } \\
\text { enthusiastic } \\
\text { about the } \\
\text { using the net }\end{array}$ & $\begin{array}{l}\text { They feel } \\
\text { fine }\end{array}$ & $\begin{array}{l}\text { When they } \\
\text { bring it } \\
\text { newly, it } \\
\text { burns my } \\
\text { skin and } \\
\text { my face, } \\
\text { but stops } \\
\text { later on. } \\
\text { Mosquitos } \\
\text { enters when } \\
\text { I go also to } \\
\text { bathroom } \\
\text { and return }\end{array}$ & $\begin{array}{l}\text { Feel fine, my } \\
\text { children are } \\
\text { using it. }\end{array}$ \\
\hline 25. & $\begin{array}{l}\text { How do you feel } \\
\text { about using bed } \\
\text { nets? }\end{array}$ & $\begin{array}{l}\text { He thinks it is } \\
\text { a good thing } \\
\text { but he has not } \\
\text { used bed net }\end{array}$ & $\begin{array}{l}\text { As for me, I } \\
\text { feel fine }\end{array}$ & $\begin{array}{l}\text { Don't like } \\
\text { it because it } \\
\text { burns his } \\
\text { skin }\end{array}$ & $\begin{array}{l}\text { I think it's good } \\
\text { but mosquito } \\
\text { not biting me, } \\
\text { so I don't use it. }\end{array}$ \\
\hline 26. & $\begin{array}{l}\text { What things do you } \\
\text { consider health risks } \\
\text { in your tribe or } \\
\text { culture? (i.e. } \\
\text { malaria, TB) }\end{array}$ & $\begin{array}{l}\text { One of the } \\
\text { things is the } \\
\text { disposal of } \\
\text { waste--waste } \\
\text { is not } \\
\text { collected. Dirt } \\
\text { pill up and } \\
\text { people. get } \\
\text { typhoid form }\end{array}$ & & Wastes & Feces and dirt \\
\hline
\end{tabular}




\begin{tabular}{|c|c|c|c|c|c|}
\hline 27. & $\begin{array}{l}\text { How do you treat } \\
\text { health risks in your } \\
\text { tribe or culture? }\end{array}$ & $\begin{array}{l}\text { One of the } \\
\text { main forms of } \\
\text { treatment is to } \\
\text { conduct the } \\
\text { education, it } \\
\text { is about } \\
\text { creating the } \\
\text { awareness of } \\
\text { the people }\end{array}$ & & $\begin{array}{l}\text { Advise } \\
\text { children to } \\
\text { bury feces } \\
\text { and wash } \\
\text { their hands }\end{array}$ & $\begin{array}{l}\text { Don't allow } \\
\text { children to toilet } \\
\text { around the } \\
\text { house because I } \\
\text { have chambers }\end{array}$ \\
\hline 28. & $\begin{array}{l}\text { How do you } \\
\text { communicate health } \\
\text { risk in your tribe or } \\
\text { culture? }\end{array}$ & $\begin{array}{l}\text { most times it } \\
\text { is radio. } \\
\text { Scarcely used } \\
\text { flyers so radio } \\
\text { and by } \\
\text { workshop }\end{array}$ & $\begin{array}{l}\text { Radio, } \\
\text { messenger, } \\
\text { word of } \\
\text { mouth }\end{array}$ & & Word of mouth \\
\hline 29. & $\begin{array}{l}\text { Do you use radio, } \\
\text { television, flyer, or } \\
\text { drum to } \\
\text { communicate health } \\
\text { risks? }\end{array}$ & & & & \\
\hline 30. & $\begin{array}{l}\text { Explain some of the } \\
\text { difficulties in } \\
\text { communicating } \\
\text { health risks in your } \\
\text { tribe or culture? }\end{array}$ & $\begin{array}{l}\text { The language } \\
\text {--like } \\
\text { condom-- will } \\
\text { be an insult. } \\
\text { To talk about } \\
\text { puberty areas } \\
\text { is not good in } \\
\text { culture }\end{array}$ & & & \\
\hline 31. & $\begin{array}{l}\text { How do the people } \\
\text { who distribute the } \\
\text { bed nets } \\
\text { communicate with } \\
\text { you? }\end{array}$ & & & & \\
\hline 32. & $\begin{array}{l}\text { Do you feel you } \\
\text { usually get clear } \\
\text { information on } \\
\text { malaria treatment } \\
\text { and health risks? }\end{array}$ & & & & \\
\hline 33. & $\begin{array}{l}\text { Whether you answer } \\
\text { yes or no, how do } \\
\text { you think health } \\
\text { risks should be } \\
\text { communicated? }\end{array}$ & & & & \\
\hline 34. & $\begin{array}{l}\text { Should your } \\
\text { language, culture, or } \\
\text { tradition matter in } \\
\text { communicating } \\
\text { health risks to you? }\end{array}$ & $\begin{array}{l}\text { Yes, it very } \\
\text { much matter. } \\
\text { The culture } \\
\text { varies. In the } \\
\text { west you can } \\
\text { talk about } \\
\text { genital areas, } \\
\text { it is a } \\
\text { problem. } \\
\text { Create a } \\
\text { problem or } \\
\text { barrier }\end{array}$ & $\begin{array}{l}\text { Language is } \\
\text { better } \\
\text { because it is } \\
\text { what we } \\
\text { understand }\end{array}$ & $\begin{array}{l}\text { Yes, I } \\
\text { prefer my } \\
\text { language } \\
\text { because I } \\
\text { understand } \\
\text { it better }\end{array}$ & $\begin{array}{l}\text { Prefers her } \\
\text { language } \\
\text { because she } \\
\text { understands it } \\
\text { better. She } \\
\text { always asks for } \\
\text { interpreter }\end{array}$ \\
\hline
\end{tabular}




\begin{tabular}{|r|l|l|l|l|l|}
\hline 35. & $\begin{array}{l}\text { If so, why and in } \\
\text { what ways? }\end{array}$ & & & \\
\hline 36. & If not, why not? & & & \\
\hline 37. & $\begin{array}{l}\text { Do you recommend } \\
\text { any changes in the } \\
\text { way information } \\
\text { about bed nets is } \\
\text { communicated? }\end{array}$ & $\begin{array}{l}\text { Yes, I think } \\
\text { there is more } \\
\text { education } \\
\text { because most } \\
\text { of our people } \\
\text { are misusing } \\
\text { the bednet. } \\
\text { Some people } \\
\text { in chicken } \\
\text { pen and some } \\
\text { use for } \\
\text { bathing }\end{array}$ & $\begin{array}{l}\text { Bednet is } \\
\text { good but } \\
\text { spray really } \\
\text { kills the } \\
\text { mosquito } \\
\text { because } \\
\text { when we } \\
\text { get out to } \\
\text { use the } \\
\text { outside } \\
\text { bathroom, } \\
\text { the } \\
\text { mosquito } \\
\text { enters }\end{array}$ & $\begin{array}{l}\text { Yes, the } \\
\text { spray is } \\
\text { better than } \\
\text { the bed nets }\end{array}$ & $\begin{array}{l}\text { Ask people to } \\
\text { continue to use } \\
\text { bed net }\end{array}$ \\
& & & \\
\end{tabular}




\begin{tabular}{|c|c|c|c|c|c|}
\hline$\#$ & Question & $\begin{array}{c}\text { Participant } \\
\# 33 \\
\end{array}$ & $\begin{array}{c}\text { Participant } \\
\text { \#34 }\end{array}$ & $\begin{array}{c}\text { Participant } \\
\text { \#35 }\end{array}$ & $\begin{array}{c}\text { Participant } \\
\# 36\end{array}$ \\
\hline 1. & Are you 18 or older? & Older male & Older female & Older male & Older man \\
\hline 2. & $\begin{array}{l}\text { If so, are you } \\
\text { between ages } 18-29 \text {, } \\
30-49,50-59 \text {, or } 60 \\
\text { and over? }\end{array}$ & $30-49$ & $50-59$ & $18-29$ & $50-59$ \\
\hline 3. & $\begin{array}{l}\text { Are you traditional } \\
\text { leader/elder, mother, } \\
\text { young adult (male or } \\
\text { female), or head of } \\
\text { household? }\end{array}$ & $\begin{array}{l}\text { Young } \\
\text { adult, hoh }\end{array}$ & $\begin{array}{l}\text { Church } \\
\text { leader }\end{array}$ & $\begin{array}{l}\text { Young adult } \\
\text { male }\end{array}$ & hoh \\
\hline 4. & $\begin{array}{l}\text { Do you live in this } \\
\text { city, village, or } \\
\text { community? }\end{array}$ & sanwein & sanwein & sanwein & Gbandi town \\
\hline 5. & $\begin{array}{l}\text { If yes, how long } \\
\text { have you lived in } \\
\text { this city, village, or } \\
\text { community? }\end{array}$ & 2 years & 30 yrs. & Born here & 3 yrs. \\
\hline 6. & $\begin{array}{l}\text { Which ethnic group } \\
\text { do you belong to? }\end{array}$ & $\begin{array}{l}\text { Bassa and } \\
\text { Kru }\end{array}$ & $\begin{array}{l}\text { Congo } \\
\text { woman but } \\
\text { mother is } \\
\text { Bassa }\end{array}$ & Bassa & Bassa \\
\hline 7. & $\begin{array}{l}\text { Which language(s) } \\
\text { do you speak? }\end{array}$ & $\begin{array}{l}\text { Bassa, Kru, } \\
\text { and English }\end{array}$ & Bassa & Bassa & $\begin{array}{l}\text { Bassa and } \\
\text { English }\end{array}$ \\
\hline 8. & $\begin{array}{l}\text { Which religion do } \\
\text { you belong to? }\end{array}$ & Christian & Christian & Christian & Christian \\
\hline 9. & $\begin{array}{l}\text { Have you ever heard } \\
\text { of malaria? }\end{array}$ & yes & yes & yes & yes \\
\hline 10. & $\begin{array}{l}\text { What is the name for } \\
\text { malaria in your local } \\
\text { language? }\end{array}$ & Sun-nee & Sun-nee & Sun-nee & Sun-nee \\
\hline 11. & $\begin{array}{l}\text { Have you ever } \\
\text { suffered from } \\
\text { malaria? }\end{array}$ & yes & yes & yes & yes \\
\hline 12. & $\begin{array}{l}\text { If no, what would } \\
\text { you do if you got } \\
\text { malaria? }\end{array}$ & & & & \\
\hline 13. & $\begin{array}{l}\text { If yes, what kinds of } \\
\text { treatments do take } \\
\text { for malaria? }\end{array}$ & Traditional & $\begin{array}{l}\text { Go to } \\
\text { hospital. } \\
\text { Knows } \\
\text { traditional } \\
\text { medicine as } \\
\text { well. } \\
\text { Certain stick } \\
\text { I the bush } \\
\text { called glob- } \\
\text { gbor }\end{array}$ & $\begin{array}{l}\text { Traditional } \\
\text { medicine. } \\
\text { Sun-gleei. }\end{array}$ & $\begin{array}{l}\text { Jologbo and } \\
\text { ganagana. } \\
\text { Yes, } \\
\text { amodiaquine }\end{array}$ \\
\hline 14. & $\begin{array}{l}\text { Do you use bed nets } \\
\text { to fight off malaria? }\end{array}$ & yes & yes & yes & no \\
\hline 15. & If not, why not? & & & & $\begin{array}{l}\text { Don’t have; } \\
\text { use spray }\end{array}$ \\
\hline 16. & $\begin{array}{l}\text { What do you use to } \\
\text { fight off malaria? }\end{array}$ & & & & spray \\
\hline
\end{tabular}




\begin{tabular}{|c|c|c|c|c|c|}
\hline 17. & Does it help? & & & & yes \\
\hline 18. & How do you know? & & & & \\
\hline 19. & $\begin{array}{l}\text { If yes, how did you } \\
\text { learn about bed nets } \\
\text { (i.e. radio, word of } \\
\text { mouth, health } \\
\text { clinic)? }\end{array}$ & $\begin{array}{l}\text { Word of } \\
\text { mouth; } \\
\text { people } \\
\text { asked them } \\
\text { to watch it } \\
\text { for } 12 \text { hours } \\
\text { before using }\end{array}$ & radio & radio & \\
\hline 20. & $\begin{array}{l}\text { Which malaria } \\
\text { treatment program } \\
\text { did you get your bed } \\
\text { nets from? }\end{array}$ & merlin & & ngo & \\
\hline 21. & $\begin{array}{l}\text { How long have you } \\
\text { used bed nets? }\end{array}$ & 2 yrs. & & & \\
\hline 22. & $\begin{array}{l}\text { Did someone teach } \\
\text { you how to use bed } \\
\text { nets? }\end{array}$ & yes & yes & yes & \\
\hline 23. & $\begin{array}{l}\text { How many people in } \\
\text { your household use } \\
\text { bed nets? }\end{array}$ & 4 & 5 & & \\
\hline 24. & $\begin{array}{l}\text { How do people in } \\
\text { your tribe or culture } \\
\text { feel about using bed } \\
\text { nets? }\end{array}$ & $\begin{array}{l}\text { Some } \\
\text { people feel } \\
\text { fine. }\end{array}$ & They use it & Very happy. & $\begin{array}{l}\text { Some people } \\
\text { do not like it } \\
\text { because it is } \\
\text { too heatly }\end{array}$ \\
\hline 25. & $\begin{array}{l}\text { How do you feel } \\
\text { about using bed } \\
\text { nets? }\end{array}$ & $\begin{array}{l}\text { He feels } \\
\text { fine }\end{array}$ & $\begin{array}{l}\text { Happy to use } \\
\text { it }\end{array}$ & Feeling fine & Don’t like it \\
\hline 26. & $\begin{array}{l}\text { What things do you } \\
\text { consider health risks } \\
\text { in your tribe or } \\
\text { culture? (i.e. malaria, } \\
\text { TB) }\end{array}$ & $\begin{array}{l}\text { Toileting } \\
\text { around, } \\
\text { dumpsite }\end{array}$ & & $\begin{array}{l}\text { Don’t keep } \\
\text { feces } \\
\text { around. }\end{array}$ & $\begin{array}{l}\text { Word of } \\
\text { mouth }\end{array}$ \\
\hline 27. & $\begin{array}{l}\text { How do you treat } \\
\text { health risks in your } \\
\text { tribe or culture? }\end{array}$ & $\begin{array}{l}\text { Clean the } \\
\text { community }\end{array}$ & & & \\
\hline 28. & $\begin{array}{l}\text { How do you } \\
\text { communicate health } \\
\text { risk in your tribe or } \\
\text { culture? }\end{array}$ & $\begin{array}{l}\text { Word of } \\
\text { mouth, } \\
\text { announce } \\
\text { from house } \\
\text { to house } \\
\end{array}$ & $\begin{array}{l}\text { Word of } \\
\text { mouth; ask } \\
\text { children to } \\
\text { bury feces }\end{array}$ & $\begin{array}{l}\text { Word of } \\
\text { mouth }\end{array}$ & \\
\hline 29. & $\begin{array}{l}\text { Do you use radio, } \\
\text { television, flyer, or } \\
\text { drum to } \\
\text { communicate health } \\
\text { risks? }\end{array}$ & & & & \\
\hline 30. & $\begin{array}{l}\text { Explain some of the } \\
\text { difficulties in } \\
\text { communicating } \\
\text { health risks in your } \\
\text { tribe or culture? }\end{array}$ & & & & \\
\hline
\end{tabular}




\begin{tabular}{|c|c|c|c|c|c|}
\hline 31. & $\begin{array}{l}\text { How do the people } \\
\text { who distribute the } \\
\text { bed nets } \\
\text { communicate with } \\
\text { you? }\end{array}$ & & & & \\
\hline 32. & $\begin{array}{l}\text { Do you feel you } \\
\text { usually get clear } \\
\text { information on } \\
\text { malaria treatment } \\
\text { and health risks? }\end{array}$ & & & & \\
\hline 33. & $\begin{array}{l}\text { Whether you answer } \\
\text { yes or no, how do } \\
\text { you think health } \\
\text { risks should be } \\
\text { communicated? }\end{array}$ & & & & \\
\hline 34. & $\begin{array}{l}\text { Should your } \\
\text { language, culture, or } \\
\text { tradition matter in } \\
\text { communicating } \\
\text { health risks to you? }\end{array}$ & $\begin{array}{l}\text { Language is } \\
\text { better } \\
\text { because I } \\
\text { understand } \\
\text { it better }\end{array}$ & $\begin{array}{l}\text { Prefers } \\
\text { language }\end{array}$ & $\begin{array}{l}\text { Yes, it is } \\
\text { important } \\
\text { and they } \\
\text { understand it }\end{array}$ & yes \\
\hline 35. & $\begin{array}{l}\text { If so, why and in } \\
\text { what ways? }\end{array}$ & $\begin{array}{l}\text { Important } \\
\text { because we } \\
\text { all } \\
\text { understand } \\
\text { it } \\
\end{array}$ & & & $\begin{array}{l}\text { Yes, because } \\
\text { I understand } \\
\text { it better In } \\
\text { my language }\end{array}$ \\
\hline 36. & If not, why not? & & & & \\
\hline 37. & $\begin{array}{l}\text { Do you recommend } \\
\text { any changes in the } \\
\text { way information } \\
\text { about bed nets is } \\
\text { communicated? }\end{array}$ & $\begin{array}{l}\text { Okay. If } \\
\text { you don't } \\
\text { wash it and } \\
\text { hang it up } \\
\text { for several } \\
\text { hours, it } \\
\text { will burn } \\
\text { you }\end{array}$ & & okay & $\begin{array}{l}\text { Too much } \\
\text { water the } \\
\text { spray. They } \\
\text { should put } \\
\text { more water }\end{array}$ \\
\hline
\end{tabular}




\begin{tabular}{|c|c|c|c|c|c|}
\hline$\#$ & Question & $\begin{array}{c}\text { Participant } \\
\# 37\end{array}$ & $\begin{array}{c}\text { Participant } \\
\# 38\end{array}$ & $\begin{array}{c}\text { Participant } \\
\text { \#39 }\end{array}$ & Participant \#40 \\
\hline 1. & $\begin{array}{l}\text { Are you } 18 \text { or } \\
\text { older? }\end{array}$ & Older female & Older female & Older male & Older \\
\hline 2. & $\begin{array}{l}\text { If so, are you } \\
\text { between ages } 18-29 \text {, } \\
30-49,50-59 \text {, or } 60 \\
\text { and over? }\end{array}$ & 60 and over & 60 and over & $\begin{array}{l}60 \text { and over } \\
\text { (73) }\end{array}$ & $50-59$ \\
\hline 3. & $\begin{array}{l}\text { Are you traditional } \\
\text { leader/elder, } \\
\text { mother, young adult } \\
\text { (male or female), or } \\
\text { head of household? }\end{array}$ & hoh & hoh & hoh & mother \\
\hline 4. & $\begin{array}{l}\text { Do you live in this } \\
\text { city, village, or } \\
\text { community? }\end{array}$ & Dark forest & Dark forest & $\begin{array}{l}\text { Dark forest } \\
\text { field }\end{array}$ & Dark forest field \\
\hline 5. & $\begin{array}{l}\text { If yes, how long } \\
\text { have you lived in } \\
\text { this city, village, or } \\
\text { community? }\end{array}$ & 2 yrs. & 10 & 1994 & 2 yrs. \\
\hline 6. & $\begin{array}{l}\text { Which ethnic group } \\
\text { do you belong to? }\end{array}$ & Bassa & Bassa & Bassa & Bassa \\
\hline 7. & $\begin{array}{l}\text { Which language(s) } \\
\text { do you speak? }\end{array}$ & Bassa & Bassa & Bassa & Bassa \\
\hline 8. & $\begin{array}{l}\text { Which religion do } \\
\text { you belong to? }\end{array}$ & Christian & Christian & Christian & Christian \\
\hline 9. & $\begin{array}{l}\text { Have you ever } \\
\text { heard of malaria? }\end{array}$ & yes & yes & yes & yes \\
\hline 10. & $\begin{array}{l}\text { What is the name } \\
\text { for malaria in your } \\
\text { local language? }\end{array}$ & Sun-nee & Sun-nee & Sun-nee & Sun-nee \\
\hline 11. & $\begin{array}{l}\text { Have you ever } \\
\text { suffered from } \\
\text { malaria? }\end{array}$ & yes & yes & yes & yes \\
\hline 12. & $\begin{array}{l}\text { If no, what would } \\
\text { you do if you got } \\
\text { malaria? }\end{array}$ & & & & \\
\hline 13. & $\begin{array}{l}\text { If yes, what kinds of } \\
\text { treatments do take } \\
\text { for malaria? }\end{array}$ & $\begin{array}{l}\text { Go to } \\
\text { hospital. }\end{array}$ & $\begin{array}{l}\text { Got to } \\
\text { hospital }\end{array}$ & $\begin{array}{l}\text { Go to } \\
\text { hospital }\end{array}$ & $\begin{array}{l}\text { Go to hospital. } \\
\text { No traditional } \\
\text { medicine }\end{array}$ \\
\hline 14. & $\begin{array}{l}\text { Do you use bed nets } \\
\text { to fight off malaria? }\end{array}$ & yes & yes & yes & yes \\
\hline 15. & If not, why not? & & & & \\
\hline 16. & $\begin{array}{l}\text { What do you use to } \\
\text { fight off malaria? }\end{array}$ & & & & \\
\hline 17. & Does it help? & & & & \\
\hline 18. & How do you know? & & & & \\
\hline 19. & $\begin{array}{l}\text { If yes, how did you } \\
\text { learn about bed nets } \\
\text { (i.e. radio, word of } \\
\text { mouth, health } \\
\text { clinic)? }\end{array}$ & $\begin{array}{l}\text { Word of } \\
\text { mouth }\end{array}$ & $\begin{array}{l}\text { Word of } \\
\text { mouth, and } \\
\text { community } \\
\text { health } \\
\text { worker }\end{array}$ & $\begin{array}{l}\text { Work of } \\
\text { mouth }\end{array}$ & $\begin{array}{l}\text { Community } \\
\text { health workers } \\
\text { divide the net }\end{array}$ \\
\hline 20. & $\begin{array}{l}\text { Which malaria } \\
\text { treatment program } \\
\text { did you get your bed } \\
\text { nets from? }\end{array}$ & Don’t know & & Don’t know & \\
\hline
\end{tabular}




\begin{tabular}{|c|c|c|c|c|c|}
\hline 21. & $\begin{array}{l}\text { How long have you } \\
\text { used bed nets? }\end{array}$ & & 4 & 5 & 2 yrs. \\
\hline 22. & $\begin{array}{l}\text { Did someone teach } \\
\text { you how to use bed } \\
\text { nets? }\end{array}$ & & & & \\
\hline 23. & $\begin{array}{l}\text { How many people } \\
\text { in your household } \\
\text { use bed nets? }\end{array}$ & & 4 & & $\begin{array}{l}1 \text { bednet in a } \\
\text { room of plenty } \\
\text { people }\end{array}$ \\
\hline 24. & $\begin{array}{l}\text { How do people in } \\
\text { your tribe or culture } \\
\text { feel about using bed } \\
\text { nets? }\end{array}$ & & $\begin{array}{l}\text { Happy but } \\
\text { the mosquito } \\
\text { always } \\
\text { comes back }\end{array}$ & happy & I am happy \\
\hline 25. & $\begin{array}{l}\text { How do you feel } \\
\text { about using bed } \\
\text { nets? }\end{array}$ & She is happy & happy & happy & Very happy \\
\hline 26. & $\begin{array}{l}\text { What things do you } \\
\text { consider health risks } \\
\text { in your tribe or } \\
\text { culture? (i.e. } \\
\text { malaria, TB) }\end{array}$ & & $\begin{array}{l}\text { Ask them to } \\
\text { clean their } \\
\text { surrounding }\end{array}$ & & \\
\hline 27. & $\begin{array}{l}\text { How do you treat } \\
\text { health risks in your } \\
\text { tribe or culture? }\end{array}$ & & & & \\
\hline 28. & $\begin{array}{l}\text { How do you } \\
\text { communicate health } \\
\text { risk in your tribe or } \\
\text { culture? }\end{array}$ & $\begin{array}{l}\text { When } \\
\text { sickness } \\
\text { catches } \\
\text { someone then } \\
\text { word spread } \\
\text { about it }\end{array}$ & & $\begin{array}{l}\text { Word of } \\
\text { mouth }\end{array}$ & Word of mouth \\
\hline 29. & $\begin{array}{l}\text { Do you use radio, } \\
\text { television, flyer, or } \\
\text { drum to } \\
\text { communicate health } \\
\text { risks? }\end{array}$ & & & & \\
\hline 30. & $\begin{array}{l}\text { Explain some of the } \\
\text { difficulties in } \\
\text { communicating } \\
\text { health risks in your } \\
\text { tribe or culture? }\end{array}$ & & & & \\
\hline 31. & $\begin{array}{l}\text { How do the people } \\
\text { who distribute the } \\
\text { bed nets } \\
\text { communicate with } \\
\text { you? }\end{array}$ & & & & \\
\hline 32. & $\begin{array}{l}\text { Do you feel you } \\
\text { usually get clear } \\
\text { information on } \\
\text { malaria treatment } \\
\text { and health risks? }\end{array}$ & & & & \\
\hline 33. & $\begin{array}{l}\text { Whether you answer } \\
\text { yes or no, how do } \\
\text { you think health } \\
\text { risks should be } \\
\text { communicated? }\end{array}$ & & & & \\
\hline
\end{tabular}




\begin{tabular}{|r|l|l|l|l|l|}
\hline 34. & $\begin{array}{l}\text { Should your } \\
\text { language, culture, or } \\
\text { tradition matter in } \\
\text { communicating } \\
\text { health risks to you? }\end{array}$ & $\begin{array}{l}\text { Understands } \\
\text { Bassa better }\end{array}$ & yes & yes \\
\hline 35. & $\begin{array}{l}\text { If so, why and in } \\
\text { what ways? }\end{array}$ & & $\begin{array}{l}\text { I understand } \\
\text { it well }\end{array}$ & $\begin{array}{c}\text { We understand } \\
\text { it better }\end{array}$ \\
\hline 36. & If not, why not? & $\begin{array}{l}\text { Do you recommend } \\
\text { any changes in the } \\
\text { way information } \\
\text { about bed nets is } \\
\text { communicated? }\end{array}$ & & & \\
\hline
\end{tabular}




\begin{tabular}{|c|c|c|c|c|c|}
\hline \# & Question & $\begin{array}{c}\text { Participant } \\
\# 41\end{array}$ & $\begin{array}{c}\text { Participant } \\
\# 42 \\
\end{array}$ & $\begin{array}{c}\text { Participant } \\
\# 43\end{array}$ & $\begin{array}{c}\text { Participant } \\
\# 44\end{array}$ \\
\hline 1. & $\begin{array}{l}\text { Are you } 18 \text { or } \\
\text { older? }\end{array}$ & Older woman & Older woman & Older woman & female \\
\hline 2. & $\begin{array}{l}\text { If so, are you } \\
\text { between ages } 18- \\
29,30-49,50-59 \text {, } \\
\text { or } 60 \text { and over? }\end{array}$ & $30-49$ & $30-49$ & $30-49$ & $18-29$ \\
\hline 3. & $\begin{array}{l}\text { Are you traditional } \\
\text { leader/elder, } \\
\text { mother, young } \\
\text { adult (male or } \\
\text { female), or head of } \\
\text { household? }\end{array}$ & mother & hoh & hoh & $\begin{array}{l}\text { Young adult } \\
\text { female }\end{array}$ \\
\hline 4. & $\begin{array}{l}\text { Do you live in this } \\
\text { city, village, or } \\
\text { community? }\end{array}$ & $\begin{array}{l}\text { Dark forest } \\
\text { field }\end{array}$ & $\begin{array}{l}\text { Dark forest } \\
\text { field }\end{array}$ & $\begin{array}{l}\text { Dark forest } \\
\text { field }\end{array}$ & $\begin{array}{l}\text { Dark forest } \\
\text { field }\end{array}$ \\
\hline 5. & $\begin{array}{l}\text { If yes, how long } \\
\text { have you lived in } \\
\text { this city, village, or } \\
\text { community? }\end{array}$ & 1993 & 15 yrs. & 2 yrs. & 2 yrs. \\
\hline 6. & $\begin{array}{l}\text { Which ethnic } \\
\text { group do you } \\
\text { belong to? }\end{array}$ & Bassa & Bassa & Bassa & Bassa \\
\hline 7. & $\begin{array}{l}\text { Which language(s) } \\
\text { do you speak? }\end{array}$ & Bassa & $\begin{array}{l}\text { Bassa and } \\
\text { English }\end{array}$ & $\begin{array}{l}\text { Bassa and } \\
\text { English }\end{array}$ & $\begin{array}{l}\text { Bassa and } \\
\text { English }\end{array}$ \\
\hline 8. & $\begin{array}{l}\text { Which religion do } \\
\text { you belong to? }\end{array}$ & Christian & Christian & Christian & Christian \\
\hline 9. & $\begin{array}{l}\text { Have you ever } \\
\text { heard of malaria? }\end{array}$ & yes & yes & yes & yes \\
\hline 10. & $\begin{array}{l}\text { What is the name } \\
\text { for malaria in your } \\
\text { local language? }\end{array}$ & Sun-nee & Sun-nee & Sun-nee & Sun-nee \\
\hline 11. & $\begin{array}{l}\text { Have you ever } \\
\text { suffered from } \\
\text { malaria? }\end{array}$ & yes & & & yes \\
\hline 12. & $\begin{array}{l}\text { If no, what would } \\
\text { you do if you got } \\
\text { malaria? }\end{array}$ & & & & \\
\hline 13. & $\begin{array}{l}\text { If yes, what kinds } \\
\text { of treatments do } \\
\text { take for malaria? }\end{array}$ & $\begin{array}{l}\text { She has } \\
\text { suffered from } \\
\text { malaria since } \\
\text { she got big; } \\
\text { only for her } \\
\text { children she } \\
\text { goes to } \\
\text { hospital }\end{array}$ & $\begin{array}{l}\text { Go to hospital. } \\
\text { Amodiaquine } \\
\text { combination }\end{array}$ & $\begin{array}{l}\text { Go to the } \\
\text { hospital. Take } \\
\text { amodiaquine; } \\
\text { used to take } \\
\text { chloroquine }\end{array}$ & amodiaquine \\
\hline 14. & $\begin{array}{l}\text { Do you use bed } \\
\text { nets to fight off } \\
\text { malaria? }\end{array}$ & & yes & yes & $\begin{array}{l}\text { Yes } \\
\text { sometimes, } \\
\text { but because } \\
\text { of the heat I } \\
\text { can use it } \\
\text { this time }\end{array}$ \\
\hline
\end{tabular}




\begin{tabular}{|c|c|c|c|c|c|}
\hline 15. & If not, why not? & & & & \\
\hline 16. & $\begin{array}{l}\text { What do you use to } \\
\text { fight off malaria? }\end{array}$ & & & & \\
\hline 17. & Does it help? & & & & \\
\hline 18. & $\begin{array}{l}\text { How do you } \\
\text { know? }\end{array}$ & & & & \\
\hline 19. & $\begin{array}{l}\text { If yes, how did you } \\
\text { learn about bed } \\
\text { nets (i.e. radio, } \\
\text { word of mouth, } \\
\text { health clinic)? }\end{array}$ & $\begin{array}{l}\text { Radio } \\
\text { directing } \\
\text { them to go to } \\
\text { certain area to } \\
\text { get bednet }\end{array}$ & $\begin{array}{l}\text { Radio, } \\
\text { community } \\
\text { health worker }\end{array}$ & $\begin{array}{l}\text { Radio, work of } \\
\text { mouth, health } \\
\text { clinic }\end{array}$ & $\begin{array}{l}\text { Radio, } \\
\text { word of } \\
\text { mouth, and } \\
\text { community } \\
\text { health } \\
\text { workers }\end{array}$ \\
\hline 20. & $\begin{array}{l}\text { Which malaria } \\
\text { treatment program } \\
\text { did you get your } \\
\text { bed nets from? }\end{array}$ & Don't know & $\begin{array}{l}\text { Merlin and } \\
\text { malaria } \\
\text { program }\end{array}$ & $\begin{array}{l}\text { First it was } \\
\text { merlin, but } \\
\text { nmcp }\end{array}$ & \\
\hline 21. & $\begin{array}{l}\text { How long have } \\
\text { you used bed nets? }\end{array}$ & & 2 yrs. & Since 2006 & 5 yrs. \\
\hline 22. & $\begin{array}{l}\text { Did someone teach } \\
\text { you how to use bed } \\
\text { nets? }\end{array}$ & $\begin{array}{l}\text { People } \\
\text { hanged nets } \\
\text { up }\end{array}$ & & $\begin{array}{l}\text { Sample on } \\
\text { paper and } \\
\text { community } \\
\text { worker }\end{array}$ & \\
\hline 23. & $\begin{array}{l}\text { How many people } \\
\text { in your household } \\
\text { use bed nets? }\end{array}$ & & $\begin{array}{l}7 \text { people using } \\
\text { two nets in one } \\
\text { room }\end{array}$ & $\begin{array}{l}5 \text {--three bed } \\
\text { nets }\end{array}$ & 2 \\
\hline 24. & $\begin{array}{l}\text { How do people in } \\
\text { your tribe or } \\
\text { culture feel about } \\
\text { using bed nets? }\end{array}$ & $\begin{array}{l}\text { Happy to } \\
\text { sleep under it }\end{array}$ & happy & $\begin{array}{l}\text { Some people } \\
\text { like it and } \\
\text { some don't like } \\
\text { it. The people } \\
\text { asked us to put } \\
\text { the bed net in } \\
\text { the sun before } \\
\text { using it; those } \\
\text { who don't do } \\
\text { say it itch their } \\
\text { skin }\end{array}$ & $\begin{array}{l}\text { Some feel } \\
\text { fine and } \\
\text { some don't } \\
\text { feel fine. } \\
\text { They feel } \\
\text { the net can } \\
\text { itch their } \\
\text { skin with } \\
\text { covered } \\
\text { with it; } \\
\text { other } \\
\text { people feel } \\
\text { it can save } \\
\text { them from } \\
\text { getting } \\
\text { malaria }\end{array}$ \\
\hline 25. & $\begin{array}{l}\text { How do you feel } \\
\text { about using bed } \\
\text { nets? }\end{array}$ & She is happy & happy & $\begin{array}{l}\text { Feel happy } \\
\text { because she } \\
\text { doesn't like the } \\
\text { mosquito noise } \\
\text { in her eyes }\end{array}$ & fine \\
\hline 26. & $\begin{array}{l}\text { What things do } \\
\text { you consider } \\
\text { health risks in your } \\
\text { tribe or culture? } \\
\text { (i.e. malaria, TB) }\end{array}$ & & & & Open pit \\
\hline 27. & $\begin{array}{l}\text { How do you treat } \\
\text { health risks in your } \\
\text { tribe or culture? }\end{array}$ & & & & \\
\hline
\end{tabular}




\begin{tabular}{|c|c|c|c|c|c|}
\hline 28. & $\begin{array}{l}\text { How do you } \\
\text { communicate } \\
\text { health risk in your } \\
\text { tribe or culture? }\end{array}$ & $\begin{array}{l}\text { Work of } \\
\text { mouth }\end{array}$ & $\begin{array}{l}\text { Word of } \\
\text { mouth; house } \\
\text { to house }\end{array}$ & Word of mouth & $\begin{array}{l}\text { Work of } \\
\text { mouth; on } \\
\text { to those I } \\
\text { know }\end{array}$ \\
\hline 29. & $\begin{array}{l}\text { Do you use radio, } \\
\text { television, flyer, or } \\
\text { drum to } \\
\text { communicate } \\
\text { health risks? }\end{array}$ & & & & \\
\hline 30. & $\begin{array}{l}\text { Explain some of } \\
\text { the difficulties in } \\
\text { communicating } \\
\text { health risks in your } \\
\text { tribe or culture? }\end{array}$ & & & & \\
\hline 31. & $\begin{array}{l}\text { How do the people } \\
\text { who distribute the } \\
\text { bed nets } \\
\text { communicate with } \\
\text { you? }\end{array}$ & & & & \\
\hline 32. & $\begin{array}{l}\text { Do you feel you } \\
\text { usually get clear } \\
\text { information on } \\
\text { malaria treatment } \\
\text { and health risks? }\end{array}$ & & & & yes \\
\hline 33. & $\begin{array}{l}\text { Whether you } \\
\text { answer yes or no, } \\
\text { how do you think } \\
\text { health risks should } \\
\text { be communicated? }\end{array}$ & & $\begin{array}{l}\text { Loud speaker } \\
\text { in community } \\
\text { outreach }\end{array}$ & & $\begin{array}{l}\text { Don't } \\
\text { know }\end{array}$ \\
\hline 34. & $\begin{array}{l}\text { Should your } \\
\text { language, culture, } \\
\text { or tradition matter } \\
\text { in communicating } \\
\text { health risks to } \\
\text { you? }\end{array}$ & yes & yes & yes & yes \\
\hline 35. & $\begin{array}{l}\text { If so, why and in } \\
\text { what ways? }\end{array}$ & $\begin{array}{l}\text { I understand it } \\
\text { better }\end{array}$ & $\begin{array}{l}\text { Because what } \\
\text { you understand } \\
\text { what you have } \\
\text { interest in. }\end{array}$ & $\begin{array}{l}\text { My language is } \\
\text { more important } \\
\text { because it is } \\
\text { my tribe and I } \\
\text { understand it } \\
\text { better }\end{array}$ & $\begin{array}{l}\text { Because I } \\
\text { understand } \\
\text { it clearly }\end{array}$ \\
\hline 36. & If not, why not? & & & & \\
\hline 37. & $\begin{array}{l}\text { Do you } \\
\text { recommend any } \\
\text { changes in the way } \\
\text { information about } \\
\text { bed nets is } \\
\text { communicated? }\end{array}$ & & okay & $\begin{array}{l}\text { I prefer the bed } \\
\text { net but the } \\
\text { spray brings } \\
\text { mosquito; keep } \\
\text { the child under } \\
\text { the bed net } \\
\text { because once } \\
\text { mosquito bites } \\
\text { him he get } \\
\text { malaria }\end{array}$ & okay \\
\hline
\end{tabular}




\begin{tabular}{|c|c|c|c|c|c|}
\hline$\#$ & Question & $\begin{array}{c}\text { Participant } \\
\text { \#45 }\end{array}$ & $\begin{array}{c}\text { Participant } \\
\text { \#46 }\end{array}$ & $\begin{array}{c}\text { Participant } \\
\# 47\end{array}$ & $\begin{array}{c}\text { Participant } \\
\text { \#48 }\end{array}$ \\
\hline 1. & $\begin{array}{l}\text { Are you } 18 \text { or } \\
\text { older? }\end{array}$ & Older female & Exact male & Older woman & Older woman \\
\hline 2. & $\begin{array}{l}\text { If so, are you } \\
\text { between ages 18- } \\
29,30-49,50-59 \text {, } \\
\text { or } 60 \text { and over? }\end{array}$ & $30-49$ & $18-29$ & $30-49$ & $30-49$ \\
\hline 3. & $\begin{array}{l}\text { Are you traditional } \\
\text { leader/elder, } \\
\text { mother, young } \\
\text { adult (male or } \\
\text { female), or head of } \\
\text { household? }\end{array}$ & mother & $\begin{array}{l}\text { Young adult } \\
\text { male }\end{array}$ & mother & mother \\
\hline 4. & $\begin{array}{l}\text { Do you live in this } \\
\text { city, village, or } \\
\text { community? }\end{array}$ & $\begin{array}{l}\text { Dark forest } \\
\text { field }\end{array}$ & $\begin{array}{l}\text { Dark forest } \\
\text { field }\end{array}$ & $\begin{array}{l}\text { Joezohn } \\
\text { community }\end{array}$ & $\begin{array}{l}\text { Joezohn } \\
\text { community }\end{array}$ \\
\hline 5. & $\begin{array}{l}\text { If yes, how long } \\
\text { have you lived in } \\
\text { this city, village, or } \\
\text { community? }\end{array}$ & 10 years & 6 yrs. & 6 yrs. & 2 yrs. \\
\hline 6. & $\begin{array}{l}\text { Which ethnic } \\
\text { group do you } \\
\text { belong to? }\end{array}$ & Kpelle & Kpelle & Bassa & Bassa \\
\hline 7. & $\begin{array}{l}\text { Which language(s) } \\
\text { do you speak? }\end{array}$ & $\begin{array}{l}\text { Kpelle and } \\
\text { English }\end{array}$ & English only & $\begin{array}{l}\text { Bassa and } \\
\text { English }\end{array}$ & $\begin{array}{l}\text { Bassa and } \\
\text { English }\end{array}$ \\
\hline 8. & $\begin{array}{l}\text { Which religion do } \\
\text { you belong to? }\end{array}$ & Christian & Christian & Christian & Christian \\
\hline 9. & $\begin{array}{l}\text { Have you ever } \\
\text { heard of malaria? }\end{array}$ & yes & yes & yes & yes \\
\hline 10. & $\begin{array}{l}\text { What is the name } \\
\text { for malaria in your } \\
\text { local language? }\end{array}$ & Gbeley-yan & Don't know & Sun-nee & Sun-nee \\
\hline 11. & $\begin{array}{l}\text { Have you ever } \\
\text { suffered from } \\
\text { malaria? }\end{array}$ & yes & yes & yes & yes \\
\hline 12. & $\begin{array}{l}\text { If no, what would } \\
\text { you do if you got } \\
\text { malaria? }\end{array}$ & & & & \\
\hline 13. & $\begin{array}{l}\text { If yes, what kinds } \\
\text { of treatments do } \\
\text { take for malaria? }\end{array}$ & $\begin{array}{l}\text { Before I } \\
\text { used to take } \\
\text { chlroquinne. } \\
\text { But take } \\
\text { ACT }\end{array}$ & amodiaquine & $\begin{array}{l}\text { Quinine, } \\
\text { amodiaquine, } \\
\text { and } \\
\text { chloroquine. } \\
\text { Not } \\
\text { traditional } \\
\text { medicine } \\
\end{array}$ & $\begin{array}{l}\text { Medical and } \\
\text { country. } \\
\text { Amodiaquine, } \\
\text { if not better, I } \\
\text { used jologbo } \\
\text { and ganagana }\end{array}$ \\
\hline 14. & $\begin{array}{l}\text { Do you use bed } \\
\text { nets to fight off } \\
\text { malaria? }\end{array}$ & yes & yes & $\begin{array}{l}\text { Yes, I use to } \\
\text { used it }\end{array}$ & \\
\hline 15. & If not, why not? & & & & \\
\hline 16. & $\begin{array}{l}\text { What do you use to } \\
\text { fight off malaria? }\end{array}$ & & & & \\
\hline 17. & Does it help? & & & & \\
\hline 18. & $\begin{array}{l}\text { How do you } \\
\text { know? }\end{array}$ & & & & \\
\hline
\end{tabular}




\begin{tabular}{|c|c|c|c|c|c|}
\hline 19. & $\begin{array}{l}\text { If yes, how did you } \\
\text { learn about bed } \\
\text { nets (i.e. radio, } \\
\text { word of mouth, } \\
\text { health clinic)? }\end{array}$ & $\begin{array}{l}\text { Word of } \\
\text { mouth and } \\
\text { community } \\
\text { health } \\
\text { worker }\end{array}$ & $\begin{array}{l}\text { Word of } \\
\text { mouth }\end{array}$ & $\begin{array}{l}\text { Word of } \\
\text { mouth }\end{array}$ & Health clinic \\
\hline 20. & $\begin{array}{l}\text { Which malaria } \\
\text { treatment program } \\
\text { did you get your } \\
\text { bed nets from? }\end{array}$ & Don't know & Don’t know & Don’t know & $\begin{array}{l}\text { County health } \\
\text { clinic }\end{array}$ \\
\hline 21. & $\begin{array}{l}\text { How long have } \\
\text { you used bed nets? }\end{array}$ & 2 yrs. & 2 yrs. & 2 yrs. & 3 yrs. \\
\hline 22. & $\begin{array}{l}\text { Did someone teach } \\
\text { you how to use bed } \\
\text { nets? }\end{array}$ & $\begin{array}{l}\text { Yes } \\
\text { community } \\
\text { workers }\end{array}$ & $\begin{array}{l}\text { Yes, the } \\
\text { community } \\
\text { workers }\end{array}$ & yes & yes \\
\hline 23. & $\begin{array}{l}\text { How many people } \\
\text { in your household } \\
\text { use bed nets? }\end{array}$ & 3 & 6 & Don't know & 5 \\
\hline 24. & $\begin{array}{l}\text { How do people in } \\
\text { your tribe or } \\
\text { culture feel about } \\
\text { using bed nets? }\end{array}$ & $\begin{array}{l}\text { Feel good } \\
\text { because you } \\
\text { can be saved } \\
\text { from malaria }\end{array}$ & $\begin{array}{l}\text { Feel good } \\
\text { because it } \\
\text { helps them at } \\
\text { times }\end{array}$ & $\begin{array}{l}\text { Feel that its } \\
\text { fine. }\end{array}$ & $\begin{array}{l}\text { Fine. We } \\
\text { never used to } \\
\text { net and get } \\
\text { mosquito but } \\
\text { bed net we } \\
\text { can't get } \\
\text { malaria }\end{array}$ \\
\hline 25. & $\begin{array}{l}\text { How do you feel } \\
\text { about using bed } \\
\text { nets? }\end{array}$ & I feel good & I feel fine & $\begin{array}{l}\text { Not feeling } \\
\text { fine }\end{array}$ & Feel good \\
\hline 26. & $\begin{array}{l}\text { What things do } \\
\text { you consider } \\
\text { health risks in your } \\
\text { tribe or culture? } \\
\text { (i.e. malaria, TB) }\end{array}$ & $\begin{array}{l}\text { Anything } \\
\text { dangerous; } \\
\text { open latrines }\end{array}$ & $\begin{array}{l}\text { Anything that } \\
\text { is bad; } \\
\text { garbage }\end{array}$ & Don’t know & $\begin{array}{l}\text { Dumpsite and } \\
\text { flies }\end{array}$ \\
\hline 27. & $\begin{array}{l}\text { How do you treat } \\
\text { health risks in your } \\
\text { tribe or culture? }\end{array}$ & & $\begin{array}{l}\text { Cover holes, } \\
\text { clean area, } \\
\text { and prevent } \\
\text { children }\end{array}$ & & $\begin{array}{l}\text { Burn } \\
\text { dumpsite to } \\
\text { stop flies }\end{array}$ \\
\hline 28. & $\begin{array}{l}\text { How do you } \\
\text { communicate } \\
\text { health risk in your } \\
\text { tribe or culture? }\end{array}$ & $\begin{array}{l}\text { Word of } \\
\text { mouth only } \\
\text { to } \\
\text { community } \\
\text { friends }\end{array}$ & $\begin{array}{l}\text { Word of } \\
\text { mouth }\end{array}$ & $\begin{array}{l}\text { Word of } \\
\text { mouth; walk } \\
\text { over }\end{array}$ & $\begin{array}{l}\text { Word of } \\
\text { mouth, walk } \\
\text { house to } \\
\text { house }\end{array}$ \\
\hline 29. & $\begin{array}{l}\text { Do you use radio, } \\
\text { television, flyer, or } \\
\text { drum to } \\
\text { communicate } \\
\text { health risks? }\end{array}$ & & & & \\
\hline 30. & $\begin{array}{l}\text { Explain some of } \\
\text { the difficulties in } \\
\text { communicating } \\
\text { health risks in your } \\
\text { tribe or culture? }\end{array}$ & & & & \\
\hline 31. & $\begin{array}{l}\text { How do the people } \\
\text { who distribute the } \\
\text { bed nets } \\
\text { communicate with } \\
\text { you? }\end{array}$ & & & & \\
\hline
\end{tabular}




\begin{tabular}{|c|c|c|c|c|c|}
\hline 32. & $\begin{array}{l}\text { Do you feel you } \\
\text { usually get clear } \\
\text { information on } \\
\text { malaria treatment } \\
\text { and health risks? }\end{array}$ & & & & \\
\hline 33. & $\begin{array}{l}\text { Whether you } \\
\text { answer yes or no, } \\
\text { how do you think } \\
\text { health risks should } \\
\text { be communicated? }\end{array}$ & & & & \\
\hline 34. & $\begin{array}{l}\text { Should your } \\
\text { language, culture, } \\
\text { or tradition matter } \\
\text { in communicating } \\
\text { health risks to } \\
\text { you? }\end{array}$ & yes & yes & yes & yes \\
\hline 35. & $\begin{array}{l}\text { If so, why and in } \\
\text { what ways? }\end{array}$ & $\begin{array}{l}\text { It is } \\
\text { important to } \\
\text { me }\end{array}$ & $\begin{array}{l}\text { Reasons is } \\
\text { that most } \\
\text { people don't } \\
\text { understand } \\
\text { English and } \\
\text { they will } \\
\text { understand it } \\
\text { better }\end{array}$ & $\begin{array}{l}\text { Maybe other } \\
\text { people can't } \\
\text { understand } \\
\text { English, so } \\
\text { you need to } \\
\text { put it in your } \\
\text { language }\end{array}$ & $\begin{array}{l}\text { Because I } \\
\text { understand it } \\
\text { well }\end{array}$ \\
\hline 36. & If not, why not? & & & & \\
\hline 37. & $\begin{array}{l}\text { Do you } \\
\text { recommend any } \\
\text { changes in the way } \\
\text { information about } \\
\text { bed nets is } \\
\text { communicated? }\end{array}$ & $\begin{array}{l}\text { I don't have } \\
\text { interest in } \\
\text { the spray } \\
\text { because the } \\
\text { spray } \\
\text { brought } \\
\text { more } \\
\text { mosquitos. I } \\
\text { trust the bed } \\
\text { net more } \\
\text { than the } \\
\text { spray }\end{array}$ & okay & $\begin{array}{l}\text { Not satisfied. } \\
\text { Suggest it } \\
\text { should be } \\
\text { changed. } \\
\text { Don't know } \\
\text { how. }\end{array}$ & $\begin{array}{l}\text { When the } \\
\text { chemical is } \\
\text { finished from } \\
\text { the net, the } \\
\text { mosquito } \\
\text { comes again. } \\
\text { Educate on } \\
\text { how long the } \\
\text { net should last }\end{array}$ \\
\hline
\end{tabular}




\begin{tabular}{|c|c|c|c|c|c|}
\hline$\#$ & Question & $\begin{array}{c}\text { Participant } \\
\# 49 \\
\end{array}$ & $\begin{array}{c}\text { Participant } \\
\# 50 \\
\end{array}$ & $\begin{array}{c}\text { Participant } \\
\# 51 \\
\end{array}$ & Participant \#52 \\
\hline 1. & $\begin{array}{l}\text { Are you } 18 \text { or } \\
\text { older? }\end{array}$ & Older male & Older male & Older woman & female \\
\hline 2. & $\begin{array}{l}\text { If so, are you } \\
\text { between ages 18- } \\
29,30-49,50-59 \text {, } \\
\text { or } 60 \text { and over? }\end{array}$ & $18-29$ & $18-29$ & $30-49$ & $30-49$ \\
\hline 3. & $\begin{array}{l}\text { Are you traditional } \\
\text { leader/elder, } \\
\text { mother, young } \\
\text { adult (male or } \\
\text { female), or head of } \\
\text { household? }\end{array}$ & $\begin{array}{l}\text { Young adult } \\
\text { male }\end{array}$ & $\begin{array}{l}\text { Young adult } \\
\text { male }\end{array}$ & mother & mother \\
\hline 4. & $\begin{array}{l}\text { Do you live in this } \\
\text { city, village, or } \\
\text { community? }\end{array}$ & Jay zohn & Jozohn & Jozohn & Jozohn \\
\hline 5. & $\begin{array}{l}\text { If yes, how long } \\
\text { have you lived in } \\
\text { this city, village, or } \\
\text { community? }\end{array}$ & 5 yrs. & 9 yrs. & 4 yrs. & 2 yrs. \\
\hline 6. & $\begin{array}{l}\text { Which ethnic } \\
\text { group do you } \\
\text { belong to? }\end{array}$ & Mandingo & Bassa & Bassa & Bassa \\
\hline 7. & $\begin{array}{l}\text { Which language(s) } \\
\text { do you speak? }\end{array}$ & $\begin{array}{l}\text { Mandingo, } \\
\text { lorma, and } \\
\text { English }\end{array}$ & $\begin{array}{l}\text { Bassa and } \\
\text { English }\end{array}$ & $\begin{array}{l}\text { Bassa and } \\
\text { English }\end{array}$ & $\begin{array}{l}\text { Bassa and } \\
\text { English }\end{array}$ \\
\hline 8. & $\begin{array}{l}\text { Which religion do } \\
\text { you belong to? }\end{array}$ & Muslim & Christian & Christian & Christian \\
\hline 9. & $\begin{array}{l}\text { Have you ever } \\
\text { heard of malaria? }\end{array}$ & yes & yes & yes & yes \\
\hline 10. & $\begin{array}{l}\text { What is the name } \\
\text { for malaria in your } \\
\text { local language? }\end{array}$ & $\begin{array}{l}\text { Gelegbanee } \\
\text { (Mandingo) }\end{array}$ & Sun-nee & Sun-nee & Sun-nee \\
\hline 11. & $\begin{array}{l}\text { Have you ever } \\
\text { suffered from } \\
\text { malaria? }\end{array}$ & yes & no & yes & yes \\
\hline 12. & $\begin{array}{l}\text { If no, what would } \\
\text { you do if you got } \\
\text { malaria? }\end{array}$ & & & & \\
\hline 13. & $\begin{array}{l}\text { If yes, what kinds } \\
\text { of treatments do } \\
\text { take for malaria? }\end{array}$ & $\begin{array}{l}\text { Amodiaquine } \\
\text { only }\end{array}$ & & $\begin{array}{l}\text { Amodiaquine, } \\
\text { quinine, } \\
\text { paracetemol; } \\
\text { yes } \\
\text { sometimes } \\
\text { she uses } \\
\text { traditional } \\
\text { medicine }\end{array}$ & amodiaquine \\
\hline 14. & $\begin{array}{l}\text { Do you use bed } \\
\text { nets to fight off } \\
\text { malaria? }\end{array}$ & & yes & $\begin{array}{l}\text { Yes, but not } \\
\text { constantly }\end{array}$ & no \\
\hline 15. & If not, why not? & & & & Just don't like it \\
\hline 16. & $\begin{array}{l}\text { What do you use to } \\
\text { fight off malaria? }\end{array}$ & yes & yes & & Mosquito spray \\
\hline
\end{tabular}




\begin{tabular}{|c|c|c|c|c|c|}
\hline 17. & Does it help? & & & & yes \\
\hline 18. & How do you know? & & & & $\begin{array}{l}\text { Kills } \\
\text { mosquito }\end{array}$ \\
\hline 19. & $\begin{array}{l}\text { If yes, how did you } \\
\text { learn about bed } \\
\text { nets (i.e. radio, } \\
\text { word of mouth, } \\
\text { health clinic)? }\end{array}$ & Health clinic & radio & radio & \\
\hline 20. & $\begin{array}{l}\text { Which malaria } \\
\text { treatment program } \\
\text { did you get your } \\
\text { bed nets from? }\end{array}$ & $\begin{array}{l}\text { Malaria } \\
\text { control }\end{array}$ & emi & $\begin{array}{l}\text { National } \\
\text { malaria } \\
\text { control }\end{array}$ & \\
\hline 21. & $\begin{array}{l}\text { How long have you } \\
\text { used bed nets? }\end{array}$ & 3 & 2 yrs. & & \\
\hline 22. & $\begin{array}{l}\text { Did someone teach } \\
\text { you how to use bed } \\
\text { nets? }\end{array}$ & yes & yes & yes & \\
\hline 23. & $\begin{array}{l}\text { How many people } \\
\text { in your household } \\
\text { use bed nets? }\end{array}$ & 5 & 3 & 4 & 4 \\
\hline 24. & $\begin{array}{l}\text { How do people in } \\
\text { your tribe or } \\
\text { culture feel about } \\
\text { using bed nets? }\end{array}$ & fine & Feel good & Feel fine & Feel fine. \\
\hline 25. & $\begin{array}{l}\text { How do you feel } \\
\text { about using bed } \\
\text { nets? }\end{array}$ & fine & Feel fine & Feel fine & $\begin{array}{l}\text { Alright, used } \\
\text { it before }\end{array}$ \\
\hline 26. & $\begin{array}{l}\text { What things do you } \\
\text { consider health } \\
\text { risks in your tribe } \\
\text { or culture? (i.e. } \\
\text { malaria, TB) }\end{array}$ & Open toilet & $\begin{array}{l}\text { Dumpsite and } \\
\text { old toilet }\end{array}$ & $\begin{array}{l}\text { Open toilet. } \\
\text { Fries from } \\
\text { toilet sit on } \\
\text { the food. And } \\
\text { open } \\
\text { bathroom }\end{array}$ & $\begin{array}{l}\text { Dump piles } \\
\text { and toilets }\end{array}$ \\
\hline 27. & $\begin{array}{l}\text { How do you treat } \\
\text { health risks in your } \\
\text { tribe or culture? }\end{array}$ & $\begin{array}{l}\text { buy } \\
\text { chloroquine }\end{array}$ & $\begin{array}{l}\text { Clean area and } \\
\text { burn dumpsite }\end{array}$ & & $\begin{array}{l}\text { Clean the } \\
\text { dumpsite }\end{array}$ \\
\hline 28. & $\begin{array}{l}\text { How do you } \\
\text { communicate } \\
\text { health risk in your } \\
\text { tribe or culture? }\end{array}$ & $\begin{array}{l}\text { Word of } \\
\text { mouth }\end{array}$ & Word of mouth & $\begin{array}{l}\text { Word of } \\
\text { mouth }\end{array}$ & $\begin{array}{l}\text { Word of } \\
\text { mouth; door } \\
\text { to door }\end{array}$ \\
\hline 29. & $\begin{array}{l}\text { Do you use radio, } \\
\text { television, flyer, or } \\
\text { drum to } \\
\text { communicate } \\
\text { health risks? }\end{array}$ & & & & \\
\hline 30. & $\begin{array}{l}\text { Explain some of } \\
\text { the difficulties in } \\
\text { communicating } \\
\text { health risks in your } \\
\text { tribe or culture? }\end{array}$ & & & & \\
\hline 31. & $\begin{array}{l}\text { How do the people } \\
\text { who distribute the } \\
\text { bed nets } \\
\text { communicate with } \\
\text { you? }\end{array}$ & & & & \\
\hline
\end{tabular}




\begin{tabular}{|c|c|c|c|c|c|}
\hline 32. & $\begin{array}{l}\text { Do you feel you } \\
\text { usually get clear } \\
\text { information on } \\
\text { malaria } \\
\text { treatment and } \\
\text { health risks? }\end{array}$ & & yes & & \\
\hline 33. & $\begin{array}{l}\text { Whether you } \\
\text { answer yes or } \\
\text { no, how do you } \\
\text { think health } \\
\text { risks should be } \\
\text { communicated? }\end{array}$ & & radio & & \\
\hline 34. & $\begin{array}{l}\text { Should your } \\
\text { language, } \\
\text { culture, or } \\
\text { tradition matter } \\
\text { in } \\
\text { communicating } \\
\text { health risks to } \\
\text { you? }\end{array}$ & yes & yes & yes & yes \\
\hline 35. & $\begin{array}{l}\text { If so, why and in } \\
\text { what ways? }\end{array}$ & $\begin{array}{l}\text { Because } \\
\text { some } \\
\text { people } \\
\text { don't } \\
\text { understand } \\
\text { English so } \\
\text { we use the } \\
\text { dialect }\end{array}$ & $\begin{array}{l}\text { Important } \\
\text { because some } \\
\text { people in the } \\
\text { community } \\
\text { don't } \\
\text { understand } \\
\text { English, so we } \\
\text { have to put in } \\
\text { Bassa for them } \\
\text { to understand }\end{array}$ & $\begin{array}{l}\text { Because some } \\
\text { people can't } \\
\text { understand } \\
\text { the English, } \\
\text { that's why } \\
\text { some people } \\
\text { put it In Bassa }\end{array}$ & $\begin{array}{l}\text { Sometimes } \\
\text { some people } \\
\text { don't } \\
\text { understand } \\
\text { English so } \\
\text { you have to } \\
\text { put it in Bassa }\end{array}$ \\
\hline 36. & If not, why not? & & & & \\
\hline 37. & $\begin{array}{l}\text { Do you } \\
\text { recommend any } \\
\text { changes in the } \\
\text { way information } \\
\text { about bed nets is } \\
\text { communicated? }\end{array}$ & & & $\begin{array}{l}\text { I like the way } \\
\text { they give the } \\
\text { information }\end{array}$ & okay \\
\hline
\end{tabular}




\begin{tabular}{|c|c|c|c|c|c|}
\hline \# & Question & $\begin{array}{c}\text { Participant } \\
\# 53\end{array}$ & $\begin{array}{c}\text { Participant } \\
\# 54\end{array}$ & $\begin{array}{c}\text { Participant } \\
\# 55\end{array}$ & Participant \#56 \\
\hline 1. & $\begin{array}{l}\text { Are you } 18 \text { or } \\
\text { older? }\end{array}$ & 18 female & older & Older female & male \\
\hline 2. & $\begin{array}{l}\text { If so, are you } \\
\text { between ages } 18- \\
29,30-49,50-59 \text {, } \\
\text { or } 60 \text { and over? }\end{array}$ & $18-29$ & $18-29$ & $30-49$ & $18-29$ \\
\hline 3. & $\begin{array}{l}\text { Are you } \\
\text { traditional } \\
\text { leader/elder, } \\
\text { mother, young } \\
\text { adult (male or } \\
\text { female), or head } \\
\text { of household? }\end{array}$ & $\begin{array}{l}\text { Young adult } \\
\text { female }\end{array}$ & $\begin{array}{l}\text { Young adult } \\
\text { female }\end{array}$ & mother & hoh \\
\hline 4. & $\begin{array}{l}\text { Do you live in } \\
\text { this city, village, } \\
\text { or community? }\end{array}$ & $\begin{array}{l}\text { Joezohn } \\
\text { community }\end{array}$ & Jozohn & Jozohn & Joezohn \\
\hline 5. & $\begin{array}{l}\text { If yes, how long } \\
\text { have you lived in } \\
\text { this city, village, } \\
\text { or community? }\end{array}$ & 4 yrs. & 5 yrs. & 5 yrs. & 1 week \\
\hline 6. & $\begin{array}{l}\text { Which ethnic } \\
\text { group do you } \\
\text { belong to? }\end{array}$ & Mandingo & Bassa & mano & Grebo \\
\hline 7. & $\begin{array}{l}\text { Which } \\
\text { language(s) do } \\
\text { you speak? }\end{array}$ & $\begin{array}{l}\text { Mandingo } \\
\text { and English }\end{array}$ & $\begin{array}{l}\text { Bassa and } \\
\text { English }\end{array}$ & $\begin{array}{l}\text { Mano, Bassa, } \\
\text { Gio, and } \\
\text { English }\end{array}$ & $\begin{array}{l}\text { Grebo and } \\
\text { English }\end{array}$ \\
\hline 8. & $\begin{array}{l}\text { Which religion do } \\
\text { you belong to? }\end{array}$ & Muslim & Christian & Christian & Christian \\
\hline 9. & $\begin{array}{l}\text { Have you ever } \\
\text { heard of malaria? }\end{array}$ & yes & yes & yes & yes \\
\hline 10. & $\begin{array}{l}\text { What is the name } \\
\text { for malaria in } \\
\text { your local } \\
\text { language? }\end{array}$ & Not audible & Sun-nee & nennen & Ouou (0h-oh) \\
\hline 11. & $\begin{array}{l}\text { Have you ever } \\
\text { suffered from } \\
\text { malaria? }\end{array}$ & yes & yes & yes & yes \\
\hline 12. & $\begin{array}{l}\text { If no, what would } \\
\text { you do if you got } \\
\text { malaria? }\end{array}$ & & & & \\
\hline 13. & $\begin{array}{l}\text { If yes, what kinds } \\
\text { of treatments do } \\
\text { take for malaria? }\end{array}$ & $\begin{array}{l}\text { Chlroquinne, } \\
\text { amodiaquine. } \\
\text { ganagana }\end{array}$ & $\begin{array}{l}\text { Country } \\
\text { medicine } \\
\text { (paupau } \\
\text { leave, plum } \\
\text { leave, sekou } \\
\text { toure leave; } \\
\text { quinine and } \\
\text { amodiaquine }\end{array}$ & $\begin{array}{l}\text { No tradition; } \\
\text { amodiaquine, } \\
\text { and } \\
\text { chlroquinne } \\
\text { when it was } \\
\text { in style }\end{array}$ & $\begin{array}{l}\text { Amodiaquine; no } \\
\text { traditional } \\
\text { medicine }\end{array}$ \\
\hline 14. & $\begin{array}{l}\text { Do you use bed } \\
\text { nets to fight off } \\
\text { malaria? }\end{array}$ & yes & yes & yes & yes \\
\hline 15. & If not, why not? & & & & \\
\hline 16. & $\begin{array}{l}\text { What do you use } \\
\text { to fight off } \\
\text { malaria? }\end{array}$ & & & & \\
\hline
\end{tabular}




\begin{tabular}{|c|c|c|c|c|c|}
\hline 17. & Does it help? & & & & \\
\hline 18. & $\begin{array}{l}\text { How do you } \\
\text { know? }\end{array}$ & & & & \\
\hline 19. & $\begin{array}{l}\text { If yes, how did } \\
\text { you learn about } \\
\text { bed nets (i.e. } \\
\text { radio, word of } \\
\text { mouth, health } \\
\text { clinic)? }\end{array}$ & Health clinic & Health clinic & Health clinic & radio \\
\hline 20. & $\begin{array}{l}\text { Which malaria } \\
\text { treatment } \\
\text { program did you } \\
\text { get your bed nets } \\
\text { from? }\end{array}$ & Don’t know & merlin & merlin & unicef \\
\hline 21. & $\begin{array}{l}\text { How long have } \\
\text { you used bed } \\
\text { nets? }\end{array}$ & 2 yrs. & & 5 yrs. & 5 yrs. \\
\hline 22. & $\begin{array}{l}\text { Did someone } \\
\text { teach you how to } \\
\text { use bed nets? }\end{array}$ & yes & yes & & no \\
\hline 23. & $\begin{array}{l}\text { How many people } \\
\text { in your household } \\
\text { use bed nets? }\end{array}$ & 6 & 3 & 5 & 5 \\
\hline 24. & $\begin{array}{l}\text { How do people in } \\
\text { your tribe or } \\
\text { culture feel about } \\
\text { using bed nets? }\end{array}$ & Fine & `feel happy & Feel fine & fine \\
\hline 25. & $\begin{array}{l}\text { How do you feel } \\
\text { about using bed } \\
\text { nets? }\end{array}$ & fine & happy & Feel fine & fine \\
\hline 26. & $\begin{array}{l}\text { What things do } \\
\text { you consider } \\
\text { health risks in } \\
\text { your tribe or } \\
\text { culture? (i.e. } \\
\text { malaria, TB) }\end{array}$ & $\begin{array}{l}\text { Flies, dump } \\
\text { pile }\end{array}$ & $\begin{array}{l}\text { Mosquito } \\
\text { and dirty } \\
\text { water }\end{array}$ & $\begin{array}{l}\text { Open well, } \\
\text { no special } \\
\text { place for } \\
\text { dumpsite, } \\
\text { open toiler, } \\
\text { dirty water } \\
\end{array}$ & \\
\hline 27. & $\begin{array}{l}\text { How do you treat } \\
\text { health risks in } \\
\text { your tribe or } \\
\text { culture? }\end{array}$ & & & clean & \\
\hline 28. & $\begin{array}{l}\text { How do you } \\
\text { communicate } \\
\text { health risk in your } \\
\text { tribe or culture? }\end{array}$ & $\begin{array}{l}\text { Word of } \\
\text { mouth; walk } \\
\text { house to } \\
\text { house }\end{array}$ & $\begin{array}{l}\text { Word of } \\
\text { mouth }\end{array}$ & Door to door & Door to door \\
\hline 29. & $\begin{array}{l}\text { Do you use radio, } \\
\text { television, flyer, } \\
\text { or drum to } \\
\text { communicate } \\
\text { health risks? }\end{array}$ & & & & \\
\hline 30. & $\begin{array}{l}\text { Explain some of } \\
\text { the difficulties in } \\
\text { communicating } \\
\text { health risks in } \\
\text { your tribe or } \\
\text { culture? }\end{array}$ & & & no & \\
\hline
\end{tabular}




\begin{tabular}{|c|c|c|c|c|c|}
\hline 31. & $\begin{array}{l}\text { How do the } \\
\text { people who } \\
\text { distribute the bed } \\
\text { nets communicate } \\
\text { with you? }\end{array}$ & & & & \\
\hline 32. & $\begin{array}{l}\text { Do you feel you } \\
\text { usually get clear } \\
\text { information on } \\
\text { malaria treatment } \\
\text { and health risks? }\end{array}$ & yes & & $\begin{array}{l}\text { No; when the } \\
\text { y gave the } \\
\text { net they } \\
\text { didn't teach } \\
\text { us how to } \\
\text { hang it up }\end{array}$ & \\
\hline 33. & $\begin{array}{l}\text { Whether you } \\
\text { answer yes or no, } \\
\text { how do you think } \\
\text { health risks } \\
\text { should be } \\
\text { communicated? }\end{array}$ & & & & \\
\hline 34. & $\begin{array}{l}\text { Should your } \\
\text { language, culture, } \\
\text { or tradition matter } \\
\text { in communicating } \\
\text { health risks to } \\
\text { you? }\end{array}$ & yes & yes & yes & yes \\
\hline 35. & $\begin{array}{l}\text { If so, why and in } \\
\text { what ways? }\end{array}$ & $\begin{array}{l}\text { Some people } \\
\text { can't } \\
\text { understand } \\
\text { English, so } \\
\text { Mandingo is } \\
\text { fine }\end{array}$ & 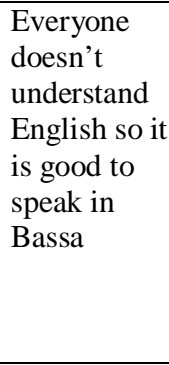 & $\begin{array}{l}\text { Then } \\
\text { everybody } \\
\text { can } \\
\text { understand } \\
\text { it; even those } \\
\text { of us who } \\
\text { speak } \\
\text { English don't } \\
\text { understand } \\
\text { some }\end{array}$ & $\begin{array}{l}\text { Because some } \\
\text { people can't } \\
\text { understand } \\
\text { English }\end{array}$ \\
\hline 36. & If not, why not? & & & & \\
\hline 37. & $\begin{array}{l}\text { Do you } \\
\text { recommend any } \\
\text { changes in the } \\
\text { way information } \\
\text { about bed nets is } \\
\text { communicated? }\end{array}$ & & & $\begin{array}{l}\text { Use more } \\
\text { door-to-radio } \\
\text { and health } \\
\text { clinic }\end{array}$ & okay \\
\hline
\end{tabular}




\begin{tabular}{|c|c|c|c|c|c|}
\hline \# & Question & $\begin{array}{c}\text { Participant } \\
\# 57\end{array}$ & $\begin{array}{c}\text { Participant } \\
\# 58\end{array}$ & $\begin{array}{c}\text { Participant } \\
\# 59\end{array}$ & $\begin{array}{c}\text { Participant } \\
\text { \#60 }\end{array}$ \\
\hline 1. & $\begin{array}{l}\text { Are you } 18 \text { or } \\
\text { older? }\end{array}$ & Older female & male & Older male & Older male \\
\hline 2. & $\begin{array}{l}\text { If so, are you } \\
\text { between ages 18- } \\
29,30-49,50-59 \text {, } \\
\text { or } 60 \text { and over? }\end{array}$ & $30-49$ & $18-29$ & $30-49$ & $50-59$ \\
\hline 3. & $\begin{array}{l}\text { Are you } \\
\text { traditional } \\
\text { leader/elder, } \\
\text { mother, young } \\
\text { adult (male or } \\
\text { female), or head } \\
\text { of household? }\end{array}$ & mother & $\begin{array}{l}\text { Young adult } \\
\text { male }\end{array}$ & hoh & hoh \\
\hline 4. & $\begin{array}{l}\text { Do you live in this } \\
\text { city, village, or } \\
\text { community? }\end{array}$ & Jozohn & Jozohn & $\begin{array}{l}\text { Sugarcane } \\
\text { farm }\end{array}$ & $\begin{array}{l}\text { Open bible } \\
\text { community }\end{array}$ \\
\hline 5. & $\begin{array}{l}\text { If yes, how long } \\
\text { have you lived in } \\
\text { this city, village, } \\
\text { or community? }\end{array}$ & 10 yrs. & 1 year & 25 years & 29 yrs. \\
\hline 6. & $\begin{array}{l}\text { Which ethnic } \\
\text { group do you } \\
\text { belong to? }\end{array}$ & $\begin{array}{l}\text { Mamba } \\
\text { Bassa }\end{array}$ & Bassa & Grebo & Bassa \\
\hline 7. & $\begin{array}{l}\text { Which } \\
\text { language(s) do } \\
\text { you speak? }\end{array}$ & Bassa & $\begin{array}{l}\text { Bassa and } \\
\text { English }\end{array}$ & $\begin{array}{l}\text { Grebo and } \\
\text { English }\end{array}$ & $\begin{array}{l}\text { Bassa, Kpelle, } \\
\text { mano, Gio, and } \\
\text { English }\end{array}$ \\
\hline 8. & $\begin{array}{l}\text { Which religion do } \\
\text { you belong to? }\end{array}$ & Christian & Christian & Christian & Christian \\
\hline 9. & $\begin{array}{l}\text { Have you ever } \\
\text { heard of malaria? }\end{array}$ & yes & yes & yes & yes \\
\hline 10. & $\begin{array}{l}\text { What is the name } \\
\text { for malaria in } \\
\text { your local } \\
\text { language? }\end{array}$ & Sun-nee & Sun-nee & neebruen & Sun-nee \\
\hline 11. & $\begin{array}{l}\text { Have you ever } \\
\text { suffered from } \\
\text { malaria? }\end{array}$ & $\begin{array}{l}\text { Yes plenty } \\
\text { time }\end{array}$ & yes & yes & yes \\
\hline 12. & $\begin{array}{l}\text { If no, what would } \\
\text { you do if you got } \\
\text { malaria? }\end{array}$ & & & & \\
\hline 13. & $\begin{array}{l}\text { If yes, what kinds } \\
\text { of treatments do } \\
\text { take for malaria? }\end{array}$ & $\begin{array}{l}\text { Traditional } \\
\text { medicine, } \\
\text { chlroquinne, } \\
\text { amodiaquine, } \\
\text { paracetemol }\end{array}$ & $\begin{array}{l}\text { Act, } \\
\text { paracetemol; } \\
\text { no traditional } \\
\text { medicine }\end{array}$ & $\begin{array}{l}\text { Western } \\
\text { medicine; } \\
\text { chlroquinne, } \\
\text { pcn. }\end{array}$ & $\begin{array}{l}\text { Traditional } \\
\text { medicines } \\
\text { (drink and } \\
\text { rub). Once in a } \\
\text { while; just go } \\
\text { to the hospital. }\end{array}$ \\
\hline 14. & $\begin{array}{l}\text { Do you use bed } \\
\text { nets to fight off } \\
\text { malaria? }\end{array}$ & no & yes & $\begin{array}{l}\text { No (used it } \\
\text { before) }\end{array}$ & yes \\
\hline 15. & If not, why not? & & & & \\
\hline 16. & $\begin{array}{l}\text { What do you use } \\
\text { to fight off } \\
\text { malaria? }\end{array}$ & $\begin{array}{l}\text { Mosquito } \\
\text { chord }\end{array}$ & & & \\
\hline
\end{tabular}




\begin{tabular}{|c|c|c|c|c|c|}
\hline 17. & Does it help? & yes & & & \\
\hline 18. & $\begin{array}{l}\text { How do you } \\
\text { know? }\end{array}$ & $\begin{array}{l}\text { Mosquito } \\
\text { can't come in } \\
\text { the room }\end{array}$ & & & \\
\hline 19. & $\begin{array}{l}\text { If yes, how did } \\
\text { you learn about } \\
\text { bed nets (i.e. } \\
\text { radio, word of } \\
\text { mouth, health } \\
\text { clinic)? }\end{array}$ & & radio & radio & radio \\
\hline 20. & $\begin{array}{l}\text { Which malaria } \\
\text { treatment program } \\
\text { did you get your } \\
\text { bed nets from? }\end{array}$ & & merlin & Don’t know & \\
\hline 21. & $\begin{array}{l}\text { How long have } \\
\text { you used bed } \\
\text { nets? }\end{array}$ & & $1 \mathrm{yr}$. & Stopped using & 2 yrs. \\
\hline 22. & $\begin{array}{l}\text { Did someone } \\
\text { teach you how to } \\
\text { use bed nets? }\end{array}$ & & yes & & yes \\
\hline 23. & $\begin{array}{l}\text { How many people } \\
\text { in your household } \\
\text { use bed nets? }\end{array}$ & & & 5 & $\begin{array}{l}12 \text { persons } \\
\text { with } 6 \text { nets }\end{array}$ \\
\hline 24. & $\begin{array}{l}\text { How do people in } \\
\text { your tribe or } \\
\text { culture feel about } \\
\text { using bed nets? }\end{array}$ & fine & Feel very fine & Feel good. & $\begin{array}{l}\text { Most of our } \\
\text { people, } \\
\text { especially in } \\
\text { the interior, we } \\
\text { used to burn } \\
\text { palm kernel } \\
\text { and certain } \\
\text { leave, and no } \\
\text { mosquito goes } \\
\text { there. Right } \\
\text { now, palm } \\
\text { kernel can last } \\
\text { for two weeks. } \\
\text { People feel fine } \\
\text { about net } \\
\text { because it is } \\
\text { quicker }\end{array}$ \\
\hline 25. & $\begin{array}{l}\text { How do you feel } \\
\text { about using bed } \\
\text { nets? }\end{array}$ & fine & Feel very fine & Feel good & $\begin{array}{l}\text { Feel fine } \\
\text { because when } \\
\text { he gets under } \\
\text { the net he } \\
\text { doesn't } \\
\text { experience } \\
\text { mosquito }\end{array}$ \\
\hline 26. & $\begin{array}{l}\text { What things do } \\
\text { you consider } \\
\text { health risks in } \\
\text { your tribe or } \\
\text { culture? (i.e. } \\
\text { malaria, TB) }\end{array}$ & & $\begin{array}{l}\text { Garbage, } \\
\text { roaches }\end{array}$ & flies & $\begin{array}{l}\text { No problem in } \\
\text { his community; } \\
\text { public health is } \\
\text { on their back }\end{array}$ \\
\hline 27. & $\begin{array}{l}\text { How do you treat } \\
\text { health risks in } \\
\text { your tribe or } \\
\text { culture? }\end{array}$ & & & $\begin{array}{l}\text { Public health } \\
\text { worker who } \\
\text { educates } \\
\text { community }\end{array}$ & \\
\hline
\end{tabular}




\begin{tabular}{|c|c|c|c|c|c|}
\hline 28. & $\begin{array}{l}\text { How do you } \\
\text { communicate } \\
\text { health risk in your } \\
\text { tribe or culture? }\end{array}$ & & Word of mouth & $\begin{array}{l}\text { Word of } \\
\text { mouth }\end{array}$ & Word of mouth \\
\hline 29. & $\begin{array}{l}\text { Do you use radio, } \\
\text { television, flyer, } \\
\text { or drum to } \\
\text { communicate } \\
\text { health risks? }\end{array}$ & & & & \\
\hline 30. & $\begin{array}{l}\text { Explain some of } \\
\text { the difficulties in } \\
\text { communicating } \\
\text { health risks in } \\
\text { your tribe or } \\
\text { culture? }\end{array}$ & & & & \\
\hline 31. & $\begin{array}{l}\text { How do the } \\
\text { people who } \\
\text { distribute the bed } \\
\text { nets communicate } \\
\text { with you? }\end{array}$ & & & & \\
\hline 32. & $\begin{array}{l}\text { Do you feel you } \\
\text { usually get clear } \\
\text { information on } \\
\text { malaria treatment } \\
\text { and health risks? }\end{array}$ & & & & \\
\hline 33. & $\begin{array}{l}\text { Whether you } \\
\text { answer yes or no, } \\
\text { how do you think } \\
\text { health risks should } \\
\text { be } \\
\text { communicated? }\end{array}$ & & & & \\
\hline 34. & $\begin{array}{l}\text { Should your } \\
\text { language, culture, } \\
\text { or tradition matter } \\
\text { in communicating } \\
\text { health risks to } \\
\text { you? }\end{array}$ & $\begin{array}{l}\text { Yes; my } \\
\text { Bassa } \\
\text { important } \\
\text { more than } \\
\text { the English }\end{array}$ & yes & yes & yes \\
\hline 35. & $\begin{array}{l}\text { If so, why and in } \\
\text { what ways? }\end{array}$ & $\begin{array}{l}\text { I understand } \\
\text { it well }\end{array}$ & $\begin{array}{l}\text { Because some } \\
\text { of us can't } \\
\text { understand } \\
\text { English very } \\
\text { well }\end{array}$ & $\begin{array}{l}\text { Because it is } \\
\text { important }\end{array}$ & $\begin{array}{l}\text { Because I was } \\
\text { born in that } \\
\text { language, in } \\
\text { that tradition, } \\
\text { and I will hear } \\
\text { it and know the } \\
\text { detail without } \\
\text { asking you }\end{array}$ \\
\hline 36. & If not, why not? & & & & \\
\hline 37. & $\begin{array}{l}\text { Do you } \\
\text { recommend any } \\
\text { changes in the } \\
\text { way information } \\
\text { about bed nets is } \\
\text { communicated? }\end{array}$ & & & & okay \\
\hline
\end{tabular}




\begin{tabular}{|c|c|c|c|c|c|}
\hline$\#$ & Question & $\begin{array}{c}\text { Participant } \\
\# 61\end{array}$ & $\begin{array}{c}\text { Participant } \\
\# 62\end{array}$ & $\begin{array}{c}\text { Participant } \\
\# 63\end{array}$ & $\begin{array}{c}\text { Participant } \\
\text { \#64 }\end{array}$ \\
\hline 1. & $\begin{array}{l}\text { Are you } 18 \text { or } \\
\text { older? }\end{array}$ & Older male & Older male & Older male & \\
\hline 2. & $\begin{array}{l}\text { If so, are you } \\
\text { between ages } \\
18-29,30-49 \text {, } \\
50-59 \text {, or } 60 \\
\text { and over? }\end{array}$ & $30-49$ & $30-49$ & $30-49$ & $50=59$ \\
\hline 3. & $\begin{array}{l}\text { Are you } \\
\text { traditional } \\
\text { leader/elder, } \\
\text { mother, young } \\
\text { adult (male or } \\
\text { female), or } \\
\text { head of } \\
\text { household? }\end{array}$ & hoh & hoh & hoh & hoh \\
\hline 4. & $\begin{array}{l}\text { Do you live in } \\
\text { this city, } \\
\text { village, or } \\
\text { community? }\end{array}$ & $\begin{array}{l}\text { Sugarcane } \\
\text { farm }\end{array}$ & Kpelle town & $\begin{array}{l}\text { Sugarcane } \\
\text { farm }\end{array}$ & $\begin{array}{l}\text { Sugarcane } \\
\text { farm }\end{array}$ \\
\hline 5. & $\begin{array}{l}\text { If yes, how } \\
\text { long have you } \\
\text { lived in this } \\
\text { city, village, or } \\
\text { community? }\end{array}$ & 20 yrs. & 3 yrs. & 25 yrs. & 50 \\
\hline 6. & $\begin{array}{l}\text { Which ethnic } \\
\text { group do you } \\
\text { belong to? }\end{array}$ & Kru & Bassa & Kru & Bassa \\
\hline 7. & $\begin{array}{l}\text { Which } \\
\text { language(s) do } \\
\text { you speak? }\end{array}$ & English only & $\begin{array}{l}\text { Bassa and } \\
\text { English }\end{array}$ & $\begin{array}{l}\text { Bassa, Kru, } \\
\text { and English }\end{array}$ & $\begin{array}{l}\text { Bassa and } \\
\text { English }\end{array}$ \\
\hline 8. & $\begin{array}{l}\text { Which religion } \\
\text { do you belong } \\
\text { to? }\end{array}$ & Christian & Christian & Christian & Christian \\
\hline 9. & $\begin{array}{l}\text { Have you ever } \\
\text { heard of } \\
\text { malaria? }\end{array}$ & yes & yes & yes & yes \\
\hline 10. & $\begin{array}{l}\text { What is the } \\
\text { name for } \\
\text { malaria in your } \\
\text { local language? }\end{array}$ & Don’t know & Sun-nee & $\begin{array}{l}\text { Sun-nee } \\
\text { (moean in Kru) }\end{array}$ & Sun-nee \\
\hline 11. & $\begin{array}{l}\text { Have you ever } \\
\text { suffered from } \\
\text { malaria? }\end{array}$ & yes & yes & yes & $\begin{array}{l}\text { Yes, } \\
\text { sometimes }\end{array}$ \\
\hline 12. & $\begin{array}{l}\text { If no, what } \\
\text { would you do if } \\
\text { you got } \\
\text { malaria? }\end{array}$ & & & & \\
\hline
\end{tabular}




\begin{tabular}{|c|c|c|c|c|c|}
\hline 13. & $\begin{array}{l}\text { If yes, what kinds } \\
\text { of treatments do } \\
\text { take for malaria? }\end{array}$ & $\begin{array}{l}\text { Amodiaqui } \\
\text { ne; no } \\
\text { traditional } \\
\text { medicine }\end{array}$ & $\begin{array}{l}\text { Drape, } \\
\text { quinine, } \\
\text { amodiaquine }\end{array}$ & $\begin{array}{l}\text { Chlroquin } \\
\text { ne and } \\
\text { quinine; } \\
\text { no } \\
\text { traditional } \\
\text { medicine }\end{array}$ & $\begin{array}{l}\text { Normally, } \\
\text { sometimes I take } \\
\text { treatment from } \\
\text { hospital ; and } \\
\text { traditional herbs. I } \\
\text { don't go hospital } \\
\text { frequently because } \\
\text { it takes me a longer } \\
\text { time to get malaria. } \\
\text { They used to give } \\
\text { me chlroquinne. } \\
\text { Having taken the } \\
\text { new malaria drugs. } \\
\text { Dry sugarcane } \\
\text { leave, butter pearl } \\
\text { leave, boiled and } \\
\text { drink the water }\end{array}$ \\
\hline 14. & $\begin{array}{l}\text { Do you use bed } \\
\text { nets to fight off } \\
\text { malaria? }\end{array}$ & yes & yes & yes & yes \\
\hline 15. & If not, why not? & & & & \\
\hline 16. & $\begin{array}{l}\text { What do you use } \\
\text { to fight off } \\
\text { malaria? }\end{array}$ & yes & yes & yes & \\
\hline 17. & Does it help? & & & & \\
\hline 18. & $\begin{array}{l}\text { How do you } \\
\text { know? }\end{array}$ & & & & \\
\hline 19. & $\begin{array}{l}\text { If yes, how did } \\
\text { you learn about } \\
\text { bed nets (i.e. radio, } \\
\text { word of mouth, } \\
\text { health clinic)? }\end{array}$ & $\begin{array}{l}\text { Health } \\
\text { clinic and } \\
\text { radio }\end{array}$ & radio & radio & $\begin{array}{l}\text { Clinic, even though } \\
\text { he heard it on the } \\
\text { radio }\end{array}$ \\
\hline 20. & $\begin{array}{l}\text { Which malaria } \\
\text { treatment program } \\
\text { did you get your } \\
\text { bed nets from? }\end{array}$ & $\begin{array}{l}\text { Concern } \\
\text { worldwide } \\
\text { (merlin) }\end{array}$ & Nets for life & $\begin{array}{l}\text { Nets for } \\
\text { life }\end{array}$ & Nets for life \\
\hline 21. & $\begin{array}{l}\text { How long have } \\
\text { you used bed nets? }\end{array}$ & 10 yrs. & 10 yrs. & 10 yrs. & $\begin{array}{l}\text { Within the past two } \\
\text { years of } \\
\text { distribution }\end{array}$ \\
\hline 22. & $\begin{array}{l}\text { Did someone teach } \\
\text { you how to use } \\
\text { bed nets? }\end{array}$ & $\begin{array}{l}\text { Yes, we } \\
\text { were taught } \\
\text { by radio } \\
\text { and health } \\
\text { center }\end{array}$ & & $\begin{array}{l}\text { Yes, nets } \\
\text { for life } \\
\text { and other } \\
\text { ngos that } \\
\text { give nets } \\
\text { out }\end{array}$ & $\begin{array}{l}\text { Well, those that } \\
\text { brought the net } \\
\text { around taught us } \\
\text { how to hang it up }\end{array}$ \\
\hline 23. & $\begin{array}{l}\text { How many people } \\
\text { in your household } \\
\text { use bed nets? }\end{array}$ & 3 & 20 persons & 10 & $\begin{array}{l}12 \text { people in five } \\
\text { rooms }\end{array}$ \\
\hline
\end{tabular}




\begin{tabular}{|c|c|c|c|c|c|}
\hline 24. & $\begin{array}{l}\text { How do people } \\
\text { in your tribe or } \\
\text { culture feel } \\
\text { about using bed } \\
\text { nets? }\end{array}$ & $\begin{array}{l}\text { Mixed. Some } \\
\text { people say it } \\
\text { produced a lot } \\
\text { of heat and } \\
\text { make them } \\
\text { suffer from } \\
\text { suffocation; } \\
\text { those who for } \\
\text { it asked } \\
\text { windows to be } \\
\text { open; doesn't } \\
\text { know of any } \\
\text { case of } \\
\text { suffocation }\end{array}$ & $\begin{array}{l}\text { They feel } \\
\text { okay; that } \\
\text { it is } \\
\text { protective. } \\
\text { It prevents } \\
\text { malaria } \\
\text { and } \\
\text { mosquito } \\
\text { from } \\
\text { biting }\end{array}$ & $\begin{array}{l}\text { They feel } \\
\text { fine because } \\
\text { it is highly } \\
\text { protective; if } \\
\text { prevents } \\
\text { mosquito } \\
\text { bite }\end{array}$ & $\begin{array}{l}\text { They feel fine. } \\
\text { When it started } \\
\text { newly people were } \\
\text { not enjoying but } \\
\text { started reacting } \\
\text { well after feedback. } \\
\text { First they had } \\
\text { complained about } \\
\text { the head }\end{array}$ \\
\hline 25. & $\begin{array}{l}\text { How do you } \\
\text { feel about } \\
\text { using bed nets? }\end{array}$ & $\begin{array}{l}\text { It is a best } \\
\text { option for him } \\
\text { and he thinks } \\
\text { he is malaria } \\
\text { free when he } \\
\text { uses bed nets. }\end{array}$ & Feel okay & $\begin{array}{l}\text { I feel fine } \\
\text { because it } \\
\text { helps me } \\
\text { against } \\
\text { mosquito } \\
\text { bites }\end{array}$ & I feel fine \\
\hline 26. & $\begin{array}{l}\text { What things do } \\
\text { you consider } \\
\text { health risks in } \\
\text { your tribe or } \\
\text { culture? (i.e. } \\
\text { malaria, TB) }\end{array}$ & $\begin{array}{l}\text { Dumpsite, } \\
\text { dirty water } \\
\text { around }\end{array}$ & $\begin{array}{l}\text { Drainages } \\
\text {, wells, } \\
\text { unsafe } \\
\text { holes. }\end{array}$ & $\begin{array}{l}\text { Unclear } \\
\text { surroundings } \\
\text {, pit latrines, } \\
\text { open toilet } \\
\text { that attract } \\
\text { flies, dirty } \\
\text { water } \\
\end{array}$ & $\begin{array}{l}\text { Swamps and the } \\
\text { environment; we } \\
\text { don't have major } \\
\text { dumpsite in the } \\
\text { community, so } \\
\text { everyone dumps in } \\
\text { the nearby bush }\end{array}$ \\
\hline 27. & $\begin{array}{l}\text { How do you } \\
\text { treat health } \\
\text { risks in your } \\
\text { tribe or } \\
\text { culture? }\end{array}$ & $\begin{array}{l}\text { Take } \\
\text { preventive } \\
\text { measures }\end{array}$ & $\begin{array}{l}\text { City } \\
\text { governme } \\
\text { nt and } \\
\text { ngos are } \\
\text { working } \\
\text { on these }\end{array}$ & $\begin{array}{l}\text { Clean our } \\
\text { surroundings }\end{array}$ & $\begin{array}{l}\text { Sometimes we burn } \\
\text { or bury the dirt }\end{array}$ \\
\hline 28. & $\begin{array}{l}\text { How do you } \\
\text { communicate } \\
\text { health risk in } \\
\text { your tribe or } \\
\text { culture? }\end{array}$ & $\begin{array}{l}\text { Basically } \\
\text { within our } \\
\text { environment } \\
\text { we use radio } \\
\text { and word of } \\
\text { mouth. People } \\
\text { do so through } \\
\text { drama }\end{array}$ & $\begin{array}{l}\text { Phone } \\
\text { calls and } \\
\text { messenger } \\
\text { s (word of } \\
\text { mouth) }\end{array}$ & $\begin{array}{l}\text { Use } \\
\text { towncrier for } \\
\text { door to door } \\
\text { announceme } \\
\text { nt }\end{array}$ & $\begin{array}{l}\text { Through the } \\
\text { community or } \\
\text { block leader }\end{array}$ \\
\hline 29. & $\begin{array}{l}\text { Do you use } \\
\text { radio, } \\
\text { television, } \\
\text { flyer, or drum } \\
\text { to } \\
\text { communicate } \\
\text { health risks? }\end{array}$ & & & & \\
\hline
\end{tabular}




\begin{tabular}{|c|c|c|c|c|c|}
\hline 30. & $\begin{array}{l}\text { Explain some of } \\
\text { the difficulties in } \\
\text { communicating } \\
\text { health risks in your } \\
\text { tribe or culture? }\end{array}$ & & & & \\
\hline 31. & $\begin{array}{l}\text { How do the people } \\
\text { who distribute the } \\
\text { bed nets } \\
\text { communicate with } \\
\text { you? }\end{array}$ & & $\begin{array}{l}\text { Over } \\
\text { radio }\end{array}$ & & \\
\hline 32. & $\begin{array}{l}\text { Do you feel you } \\
\text { usually get clear } \\
\text { information on } \\
\text { malaria treatment } \\
\text { and health risks? }\end{array}$ & & & $\begin{array}{l}\text { Need more } \\
\text { sensitization } \\
\text { education. }\end{array}$ & clear \\
\hline 33. & $\begin{array}{l}\text { Whether you } \\
\text { answer yes or no, } \\
\text { how do you think } \\
\text { health risks should } \\
\text { be communicated? }\end{array}$ & $\begin{array}{l}\text { For our } \\
\text { environ } \\
\text { ment, it } \\
\text { should } \\
\text { be done } \\
\text { by radio } \\
\text { and } \\
\text { door-to } \\
\text { door }\end{array}$ & & $\begin{array}{l}\text { Should be by } \\
\text { radio, towncrier, } \\
\text { keep environment } \\
\text { clean. }\end{array}$ & $\begin{array}{l}\text { It should be done } \\
\text { firstly from your } \\
\text { own environment } \\
\text { because how you } \\
\text { will treat your yard } \\
\text { to keep it clear, } \\
\text { then you can tell } \\
\text { others. }\end{array}$ \\
\hline 34. & $\begin{array}{l}\text { Should your } \\
\text { language, culture, } \\
\text { or tradition matter } \\
\text { in communicating } \\
\text { health risks to } \\
\text { you? }\end{array}$ & yes & yes & Yes, certainly. & Yes, it’s important \\
\hline 35. & $\begin{array}{l}\text { If so, why and in } \\
\text { what ways? }\end{array}$ & $\begin{array}{l}\text { Importan } \\
\text { t because } \\
\text { they } \\
\text { should } \\
\text { do so } \\
\text { through } \\
\text { dialects } \\
\text { and } \\
\text { tradition } \\
\text { al plays }\end{array}$ & $\begin{array}{l}\text { It is } \\
\text { very } \\
\text { import } \\
\text { ant; } \\
\text { that } \\
\text { part of } \\
\text { culture } \\
\text {. It is } \\
\text { the } \\
\text { best } \\
\text { way to } \\
\text { have } \\
\text { someo } \\
\text { ne } \\
\text { comm } \\
\text { unicat } \\
\text { ed } \\
\text { with }\end{array}$ & $\begin{array}{l}\text { For example, the } \\
\text { Poro and Sande } \\
\text { institutions thrive } \\
\text { on the local } \\
\text { language }\end{array}$ & $\begin{array}{l}\text { The language we } \\
\text { speak you can } \\
\text { better explain to } \\
\text { the lower class and } \\
\text { they will better } \\
\text { explain; you may } \\
\text { not better explain } \\
\text { in English and the } \\
\text { people may not } \\
\text { better understand in } \\
\text { English }\end{array}$ \\
\hline 36. & If not, why not? & & & & \\
\hline
\end{tabular}




\begin{tabular}{|c|c|c|c|c|c|}
\hline 37. & $\begin{array}{l}\text { Do you } \\
\text { recommend any } \\
\text { changes in the way } \\
\text { information about } \\
\text { bed nets is } \\
\text { communicated? }\end{array}$ & $\begin{array}{l}\text { For our } \\
\text { level; I } \\
\text { think it is } \\
\text { sufficient }\end{array}$ & $\begin{array}{l}\text { It' } \\
\text { s } \\
\text { ok } \\
\text { ay }\end{array}$ & $\begin{array}{l}\text { Need to improve } \\
\text { on it, In the sense } \\
\text { that people use it } \\
\text { for fishing; } \\
\text { people should be } \\
\text { adequately } \\
\text { educated and } \\
\text { sensitive in use of } \\
\text { the net. The net is } \\
\text { not for fishing but } \\
\text { important to use } \\
\text { life }\end{array}$ & $\begin{array}{l}\text { Well, at this time I will } \\
\text { not say that it might be } \\
\text { changed, and I would } \\
\text { say it might not be } \\
\text { changed because while I } \\
\text { am saying that is that } \\
\text { Liberia is } \\
\text { underdeveloped. Like } \\
\text { developed countries, } \\
\text { you attach the base of } \\
\text { the mosquito. But here } \\
\text { we don't have anything } \\
\text { to attach the base of the } \\
\text { mosquito, which is the } \\
\text { swamp. To spray the } \\
\text { swamp and all those } \\
\text { things to get the } \\
\text { mosquito from the } \\
\text { swamps. But we are } \\
\text { only stopping them in } \\
\text { the room. But they are } \\
\text { still, they will still be } \\
\text { coming because they } \\
\text { will still be producing, } \\
\text { you see. But if you } \\
\text { attach the base that } \\
\text { means that the } \\
\text { production stops. But as } \\
\text { they are producing their } \\
\text { eggs and things, they } \\
\text { will still have to be } \\
\text { coming. But in the } \\
\text { meantime the net is is } \\
\text { alright because that's } \\
\text { the only thing that can } \\
\text { stop the mosquito urn } \\
\text { from the human being } \\
\text { now, but it cannot stop } \\
\text { the mosquito from the } \\
\text { country or from the city. } \\
\text { (tape } 7 \text { ) }\end{array}$ \\
\hline
\end{tabular}




\begin{tabular}{|c|c|c|c|c|c|}
\hline$\#$ & Question & $\begin{array}{c}\text { Participant } \\
\text { \#65 }\end{array}$ & $\begin{array}{c}\text { Participant } \\
\text { \#66 }\end{array}$ & $\begin{array}{c}\text { Participant } \\
\# 67\end{array}$ & $\begin{array}{c}\text { Participant } \\
\text { \#68 }\end{array}$ \\
\hline 1. & $\begin{array}{l}\text { Are you } 18 \text { or } \\
\text { older? }\end{array}$ & Older male & Older male & Older male & Older male \\
\hline 2. & $\begin{array}{l}\text { If so, are you } \\
\text { between ages } 18- \\
29,30-49,50-59 \text {, } \\
\text { or } 60 \text { and over? }\end{array}$ & 1940 & $30-49$ & $30-49$ & $30-49$ \\
\hline 3. & $\begin{array}{l}\text { Are you } \\
\text { traditional } \\
\text { leader/elder, } \\
\text { mother, young } \\
\text { adult (male or } \\
\text { female), or head } \\
\text { of household? }\end{array}$ & hoh & hoh & hoh & hoh \\
\hline 4. & $\begin{array}{l}\text { Do you live in } \\
\text { this city, village, } \\
\text { or community? }\end{array}$ & $\begin{array}{l}\text { Dark forest } \\
\text { field }\end{array}$ & $\begin{array}{l}\text { Dark forest } \\
\text { field }\end{array}$ & $\begin{array}{l}\text { Dark forest } \\
\text { field }\end{array}$ & $\begin{array}{l}\text { Gbediah } \\
\text { town, } \\
\text { Rivercess }\end{array}$ \\
\hline 5. & $\begin{array}{l}\text { If yes, how long } \\
\text { have you lived in } \\
\text { this city, village, } \\
\text { or community? }\end{array}$ & Since 2003 & & 20 yrs. & Born there \\
\hline 6. & $\begin{array}{l}\text { Which ethnic } \\
\text { group do you } \\
\text { belong to? }\end{array}$ & Bassa & Bassa & Bassa & Bassa \\
\hline 7. & $\begin{array}{l}\text { Which } \\
\text { language(s) do } \\
\text { you speak? }\end{array}$ & Bassa & & Bassa & Bassa \\
\hline 8. & $\begin{array}{l}\text { Which religion do } \\
\text { you belong to? }\end{array}$ & Christian & Christian & Christian & Christian \\
\hline 9. & $\begin{array}{l}\text { Have you ever } \\
\text { heard of malaria? }\end{array}$ & yes & yes & yes & yes \\
\hline 10. & $\begin{array}{l}\text { What is the name } \\
\text { for malaria in } \\
\text { your local } \\
\text { language? }\end{array}$ & Sun-nee & Sun-nee & Sun-nee & Sun-nee \\
\hline 11. & $\begin{array}{l}\text { Have you ever } \\
\text { suffered from } \\
\text { malaria? }\end{array}$ & yes & yes & yes & yes \\
\hline 12. & $\begin{array}{l}\text { If no, what would } \\
\text { you do if you got } \\
\text { malaria? }\end{array}$ & & & & \\
\hline 13. & $\begin{array}{l}\text { If yes, what kinds } \\
\text { of treatments do } \\
\text { take for malaria? }\end{array}$ & $\begin{array}{l}\text { Traditional } \\
\text { medicine. } \\
\text { Use tot, } \\
\text { plum bark, } \\
\text { sekou toure } \\
\text { root, dry } \\
\text { plaintainleaf, } \\
\text { put zarpor, } \\
\text { garzu, and } \\
\text { mix and } \\
\text { drink. }\end{array}$ & $\begin{array}{l}\text { Take western } \\
\text { medicine and } \\
\text { traditional } \\
\text { medicine. } \\
\text { Amodiaquine. } \\
\text { I know the } \\
\text { tree but I } \\
\text { don't know } \\
\text { the name }\end{array}$ & $\begin{array}{l}\text { Sometimes I } \\
\text { take } \\
\text { amodiaquine } \\
\text { and quinine. If } \\
\text { it doesn't then } \\
\text { I take tradition } \\
\text { medicine. Plum } \\
\text { leaves }\end{array}$ & $\begin{array}{l}\text { Go to } \\
\text { hospital and } \\
\text { traditional } \\
\text { treatment. } \\
\text { Amodiaquine } \\
\text { and quinine. } \\
\text { Bark of plum } \\
\text { tree and } \\
\text { other herbs }\end{array}$ \\
\hline
\end{tabular}




\begin{tabular}{|c|c|c|c|c|c|}
\hline 14. & $\begin{array}{l}\text { Do you use bed } \\
\text { nets to fight off } \\
\text { malaria? }\end{array}$ & yes & & yes & yes \\
\hline 15. & If not, why not? & & & & \\
\hline 16. & $\begin{array}{l}\text { What do you use } \\
\text { to fight off } \\
\text { malaria? }\end{array}$ & & & & \\
\hline 17. & Does it help? & & & & \\
\hline 18. & $\begin{array}{l}\text { How do you } \\
\text { know? }\end{array}$ & & & & \\
\hline 19. & $\begin{array}{l}\text { If yes, how did } \\
\text { you learn about } \\
\text { bed nets (i.e. } \\
\text { radio, word of } \\
\text { mouth, health } \\
\text { clinic)? }\end{array}$ & $\begin{array}{l}\text { Those who } \\
\text { share it }\end{array}$ & radio & $\begin{array}{l}\text { Health workers } \\
\text { and radio }\end{array}$ & $\begin{array}{l}\text { Word of } \\
\text { mouth }\end{array}$ \\
\hline 20. & $\begin{array}{l}\text { Which malaria } \\
\text { treatment program } \\
\text { did you get your } \\
\text { bed nets from? }\end{array}$ & Don't know & & $\begin{array}{l}\text { Government } \\
\text { hospital }\end{array}$ & $\begin{array}{l}\text { Those who } \\
\text { brought it }\end{array}$ \\
\hline 21. & $\begin{array}{l}\text { How long have } \\
\text { you used bed } \\
\text { nets? }\end{array}$ & & 3 yrs. & 4 yrs. & 4 \\
\hline 22. & $\begin{array}{l}\text { Did someone } \\
\text { teach you how to } \\
\text { use bed nets? }\end{array}$ & & $\begin{array}{l}\text { Those who } \\
\text { brought it }\end{array}$ & $\begin{array}{l}\text { Those who } \\
\text { brought it }\end{array}$ & $\begin{array}{l}\text { Yes, those } \\
\text { who brought } \\
\text { it }\end{array}$ \\
\hline 23. & $\begin{array}{l}\text { How many people } \\
\text { in your household } \\
\text { use bed nets? }\end{array}$ & 6 & 4 & $\begin{array}{l}11 \text { persons in } \\
\text { house }\end{array}$ & 6 \\
\hline 24. & $\begin{array}{l}\text { How do people in } \\
\text { your tribe or } \\
\text { culture feel about } \\
\text { using bed nets? }\end{array}$ & $\begin{array}{l}\text { We only use } \\
\text { it because } \\
\text { they brought } \\
\text { it for us to } \\
\text { use. Unless } \\
\text { you get } \\
\text { outside, } \\
\text { mosquito } \\
\text { will not } \\
\text { bother you }\end{array}$ & $\begin{array}{l}\text { They are very } \\
\text { happy about it }\end{array}$ & $\begin{array}{l}\text { We are happy } \\
\text { because it } \\
\text { makes } \\
\text { mosquito not to } \\
\text { burn out at } \\
\text { nigh }\end{array}$ & $\begin{array}{l}\text { Happy about } \\
\text { it }\end{array}$ \\
\hline 25. & $\begin{array}{l}\text { How do you feel } \\
\text { about using bed } \\
\text { nets? }\end{array}$ & It is okay & I feel happy & I feel happy & $\begin{array}{l}\text { I am very } \\
\text { happy also }\end{array}$ \\
\hline 26. & $\begin{array}{l}\text { What things do } \\
\text { you consider } \\
\text { health risks in } \\
\text { your tribe or } \\
\text { culture? (i.e. } \\
\text { malaria, TB) }\end{array}$ & $\begin{array}{l}\text { Don't drink } \\
\text { open well } \\
\text { water; dead } \\
\text { roaches, } \\
\text { frogs }\end{array}$ & & & \\
\hline 27. & $\begin{array}{l}\text { How do you treat } \\
\text { health risks in } \\
\text { your tribe or } \\
\text { culture? }\end{array}$ & & & & \\
\hline 28. & $\begin{array}{l}\text { How do you } \\
\text { communicate } \\
\text { health risk in your } \\
\text { tribe or culture? }\end{array}$ & $\begin{array}{l}\text { Walk to the } \\
\text { closest } \\
\text { people; word } \\
\text { of mouth }\end{array}$ & $\begin{array}{l}\text { Word of } \\
\text { mouth; I tell } \\
\text { my friends }\end{array}$ & Word of mouth & $\begin{array}{l}\text { Word of } \\
\text { mouth }\end{array}$ \\
\hline
\end{tabular}




\begin{tabular}{|c|c|c|c|c|c|}
\hline 29. & $\begin{array}{l}\text { Do you use radio, } \\
\text { television, flyer, } \\
\text { or drum to } \\
\text { communicate } \\
\text { health risks? }\end{array}$ & & & & \\
\hline 30. & $\begin{array}{l}\text { Explain some of } \\
\text { the difficulties in } \\
\text { communicating } \\
\text { health risks in } \\
\text { your tribe or } \\
\text { culture? }\end{array}$ & & & & \\
\hline 31. & $\begin{array}{l}\text { How do the } \\
\text { people who } \\
\text { distribute the bed } \\
\text { nets communicate } \\
\text { with you? }\end{array}$ & & & & \\
\hline 32. & $\begin{array}{l}\text { Do you feel you } \\
\text { usually get clear } \\
\text { information on } \\
\text { malaria treatment } \\
\text { and health risks? }\end{array}$ & & & & \\
\hline 33. & $\begin{array}{l}\text { Whether you } \\
\text { answer yes or no, } \\
\text { how do you think } \\
\text { health risks } \\
\text { should be } \\
\text { communicated? }\end{array}$ & & & & \\
\hline 34. & $\begin{array}{l}\text { Should your } \\
\text { language, culture, } \\
\text { or tradition matter } \\
\text { in communicating } \\
\text { health risks to } \\
\text { you? }\end{array}$ & yes & yes & yes & yes \\
\hline 35. & $\begin{array}{l}\text { If so, why and in } \\
\text { what ways? }\end{array}$ & $\begin{array}{l}\text { It is } \\
\text { important. I } \\
\text { am Bassa, } \\
\text { and I } \\
\text { understand } \\
\text { better if you } \\
\text { speak Bassa } \\
\text { to me than } \\
\text { English }\end{array}$ & $\begin{array}{l}\text { It is important } \\
\text { because it is } \\
\text { my } \\
\text { knowledge }\end{array}$ & $\begin{array}{l}\text { My language is } \\
\text { important } \\
\text { because that is } \\
\text { what I was } \\
\text { born in and } \\
\text { understand }\end{array}$ & $\begin{array}{l}\text { That's the } \\
\text { language I } \\
\text { born in and } \\
\text { understand }\end{array}$ \\
\hline 36. & If not, why not? & & & & \\
\hline 37. & $\begin{array}{l}\text { Do you } \\
\text { recommend any } \\
\text { changes in the } \\
\text { way information } \\
\text { about bed nets is } \\
\text { communicated? }\end{array}$ & & & & \\
\hline
\end{tabular}




\begin{tabular}{|c|c|c|c|c|c|}
\hline \# & Question & $\begin{array}{c}\text { Participant } \\
\# 69\end{array}$ & $\begin{array}{c}\text { Participant } \\
\text { \#70 }\end{array}$ & $\begin{array}{c}\text { Participant } \\
\# 71\end{array}$ & $\begin{array}{c}\text { Participant } \\
\text { \#72 }\end{array}$ \\
\hline 1. & $\begin{array}{l}\text { Are you } 18 \text { or } \\
\text { older? }\end{array}$ & Older female & Older female & Older female & Older female \\
\hline 2. & $\begin{array}{l}\text { If so, are you } \\
\text { between ages } \\
18-29,30-49 \text {, } \\
50-59 \text {, or } 60 \\
\text { and over? }\end{array}$ & $30-49$ & $30-49$ & $30-49$ & $30-49$ \\
\hline 3. & $\begin{array}{l}\text { Are you } \\
\text { traditional } \\
\text { leader/elder, } \\
\text { mother, young } \\
\text { adult (male or } \\
\text { female), or } \\
\text { head of } \\
\text { household? }\end{array}$ & Mother/ hoh & hoh & mother & mother \\
\hline 4. & $\begin{array}{l}\text { Do you live in } \\
\text { this city, } \\
\text { village, or } \\
\text { community? }\end{array}$ & District \#3c & $\begin{array}{l}\text { Dark forest } \\
\text { field }\end{array}$ & $\begin{array}{l}\text { Dark forest } \\
\text { field }\end{array}$ & $\begin{array}{l}\text { Dark forest } \\
\text { field }\end{array}$ \\
\hline 5. & $\begin{array}{l}\text { If yes, how } \\
\text { long have you } \\
\text { lived in this } \\
\text { city, village, or } \\
\text { community? }\end{array}$ & Born there & Don't know & $\begin{array}{l}\text { Don't know, } \\
\text { but I have } \\
\text { been here } \\
\text { very long }\end{array}$ & 18 yrs. \\
\hline 6. & $\begin{array}{l}\text { Which ethnic } \\
\text { group do you } \\
\text { belong to? }\end{array}$ & Bassa & Bassa & Bassa & Bassa \\
\hline 7. & $\begin{array}{l}\text { Which } \\
\text { language(s) do } \\
\text { you speak? }\end{array}$ & Bassa & Bassa & Bassa & $\begin{array}{l}\text { Bassa and } \\
\text { English }\end{array}$ \\
\hline 8. & $\begin{array}{l}\text { Which religion } \\
\text { do you belong } \\
\text { to? }\end{array}$ & Christian & Christian & Christian & Christian \\
\hline 9. & $\begin{array}{l}\text { Have you ever } \\
\text { heard of } \\
\text { malaria? }\end{array}$ & yes & yes & yes & yes \\
\hline 10. & $\begin{array}{l}\text { What is the } \\
\text { name for } \\
\text { malaria in your } \\
\text { local language? }\end{array}$ & Sun-nee & Sun-nee & Sun-nee & Sun-nee \\
\hline 11. & $\begin{array}{l}\text { Have you ever } \\
\text { suffered from } \\
\text { malaria? }\end{array}$ & yes & yes & yes & yes \\
\hline 12. & $\begin{array}{l}\text { If no, what } \\
\text { would you do } \\
\text { if you got } \\
\text { malaria? }\end{array}$ & & & & \\
\hline
\end{tabular}




\begin{tabular}{|c|c|c|c|c|c|}
\hline 13. & $\begin{array}{l}\text { If yes, what } \\
\text { kinds of } \\
\text { treatments do } \\
\text { take for } \\
\text { malaria? }\end{array}$ & $\begin{array}{l}\text { In the interior, } \\
\text { paupau root } \\
\text { and mix it with } \\
\text { leaves to drink }\end{array}$ & $\begin{array}{l}\text { Mixed. } \\
\text { Hospital and } \\
\text { traditional }\end{array}$ & $\begin{array}{l}\text { Mixed. } \\
\text { Hospital and } \\
\text { traditional. If } \\
\text { go hospital } \\
\text { and if it } \\
\text { doesn't work } \\
\text { then I take } \\
\text { traditional }\end{array}$ & $\begin{array}{l}\text { Mixed. } \\
\text { Western and } \\
\text { traditional. } \\
\text { Amodiaquine } \\
\text { and quinine. } \\
\text { Sekou toure } \\
\text { leave, plum } \\
\text { leave, paupau } \\
\text { leave, etc. }\end{array}$ \\
\hline 14. & $\begin{array}{l}\text { Do you use bed } \\
\text { nets to fight off } \\
\text { malaria? }\end{array}$ & yes & yes & yes & yes \\
\hline 15. & $\begin{array}{l}\text { If not, why } \\
\text { not? }\end{array}$ & & & & \\
\hline 16. & $\begin{array}{l}\text { What do you } \\
\text { use to fight off } \\
\text { malaria? }\end{array}$ & & & & \\
\hline 17. & Does it help? & & & & \\
\hline 18. & $\begin{array}{l}\text { How do you } \\
\text { know? }\end{array}$ & & & & \\
\hline 19. & $\begin{array}{l}\text { If yes, how did } \\
\text { you learn about } \\
\text { bed nets (i.e. } \\
\text { radio, word of } \\
\text { mouth, health } \\
\text { clinic)? }\end{array}$ & $\begin{array}{l}\text { Community } \\
\text { health workers }\end{array}$ & $\begin{array}{l}\text { Community } \\
\text { health workers }\end{array}$ & $\begin{array}{l}\text { Those who } \\
\text { brought it }\end{array}$ & $\begin{array}{l}\text { People who } \\
\text { shared it in the } \\
\text { community }\end{array}$ \\
\hline 20. & $\begin{array}{l}\text { Which malaria } \\
\text { treatment } \\
\text { program did } \\
\text { you get your } \\
\text { bed nets from? }\end{array}$ & Don't know & Don't know & Don’t know & $\begin{array}{l}\text { Government } \\
\text { hospital }\end{array}$ \\
\hline 21. & $\begin{array}{l}\text { How long have } \\
\text { you used bed } \\
\text { nets? }\end{array}$ & 3 yrs. & 3 yrs. & 4 yrs. & 4 yrs. \\
\hline 22. & $\begin{array}{l}\text { Did someone } \\
\text { teach you how } \\
\text { to use bed } \\
\text { nets? }\end{array}$ & $\begin{array}{l}\text { Yes, those who } \\
\text { brought it }\end{array}$ & $\begin{array}{l}\text { Yes, those who } \\
\text { brought it }\end{array}$ & yes & $\begin{array}{l}\text { Yes, those who } \\
\text { brought it }\end{array}$ \\
\hline 23. & $\begin{array}{l}\text { How many } \\
\text { people in your } \\
\text { household use } \\
\text { bed nets? }\end{array}$ & 8 persons & 10 persons & 2 & 10 \\
\hline 24. & $\begin{array}{l}\text { How do people } \\
\text { in your tribe or } \\
\text { culture feel } \\
\text { about using bed } \\
\text { nets? }\end{array}$ & $\begin{array}{l}\text { It is very good } \\
\text { for my people } \\
\text { and myself }\end{array}$ & $\begin{array}{l}\text { When you } \\
\text { sleep under the } \\
\text { net it is okay }\end{array}$ & $\begin{array}{l}\text { They say it is } \\
\text { fine }\end{array}$ & $\begin{array}{l}\text { They feel fine } \\
\text { because it } \\
\text { saves them } \\
\text { from the } \\
\text { mosquito } \\
\text { biting them }\end{array}$ \\
\hline 25. & $\begin{array}{l}\text { How do you } \\
\text { feel about } \\
\text { using bed nets? }\end{array}$ & $\begin{array}{l}\text { I'm very happy } \\
\text { about it }\end{array}$ & Happy about it & It is fine & $\begin{array}{l}\text { I'm happy } \\
\text { about it } \\
\text { because it } \\
\text { prevents } \\
\text { mosquito }\end{array}$ \\
\hline
\end{tabular}




\begin{tabular}{|c|c|c|c|c|c|}
\hline 26. & $\begin{array}{l}\text { What things do } \\
\text { you consider } \\
\text { health risks in } \\
\text { your tribe or } \\
\text { culture? (i.e. } \\
\text { malaria, TB) }\end{array}$ & & $\begin{array}{l}\text { Sun-nee, } \\
\text { typhoid, sore }\end{array}$ & & $\begin{array}{l}\text { Open toilet, } \\
\text { dumpsite, } \\
\text { children } \\
\text { toileting } \\
\text { everywhere }\end{array}$ \\
\hline 27. & $\begin{array}{l}\text { How do you } \\
\text { treat health } \\
\text { risks in your } \\
\text { tribe or } \\
\text { culture? }\end{array}$ & & & & \\
\hline 28. & $\begin{array}{l}\text { How do you } \\
\text { communicate } \\
\text { health risk in } \\
\text { your tribe or } \\
\text { culture? }\end{array}$ & Word of mouth & $\begin{array}{l}\text { Word of } \\
\text { mouth; } \\
\text { something like } \\
\text { that happy you } \\
\text { go and tell the } \\
\text { other people }\end{array}$ & $\begin{array}{l}\text { Word of } \\
\text { mouth }\end{array}$ & $\begin{array}{l}\text { Walking to the } \\
\text { various houses } \\
\text { and telling } \\
\text { them }\end{array}$ \\
\hline 29. & $\begin{array}{l}\text { Do you use } \\
\text { radio, } \\
\text { television, } \\
\text { flyer, or drum } \\
\text { to } \\
\text { communicate } \\
\text { health risks? }\end{array}$ & & & & \\
\hline 30. & $\begin{array}{l}\text { Explain some } \\
\text { of the } \\
\text { difficulties in } \\
\text { communicating } \\
\text { health risks in } \\
\text { your tribe or } \\
\text { culture? }\end{array}$ & & & & \\
\hline 31. & $\begin{array}{l}\text { How do the } \\
\text { people who } \\
\text { distribute the } \\
\text { bed nets } \\
\text { communicate } \\
\text { with you? }\end{array}$ & & & & \\
\hline 32. & $\begin{array}{l}\text { Do you feel } \\
\text { you usually get } \\
\text { clear } \\
\text { information on } \\
\text { malaria } \\
\text { treatment and } \\
\text { health risks? } \\
\end{array}$ & & & & \\
\hline 33. & $\begin{array}{l}\text { Whether you } \\
\text { answer yes or } \\
\text { no, how do you } \\
\text { think health } \\
\text { risks should be } \\
\text { communicated? }\end{array}$ & & & & \\
\hline
\end{tabular}




\begin{tabular}{|c|c|c|c|c|c|}
\hline 34. & $\begin{array}{l}\text { Should your } \\
\text { language, } \\
\text { culture, or } \\
\text { tradition matter } \\
\text { in } \\
\text { communicating } \\
\text { health risks to } \\
\text { you? }\end{array}$ & yes & yes & yes & yes \\
\hline 35. & $\begin{array}{l}\text { If so, why and } \\
\text { in what ways? }\end{array}$ & $\begin{array}{l}\text { Because it is } \\
\text { my native } \\
\text { language }\end{array}$ & $\begin{array}{l}\text { Because it is } \\
\text { my native } \\
\text { tongue and I } \\
\text { understand it } \\
\text { better }\end{array}$ & $\begin{array}{l}\text { Because I am } \\
\text { Bassa }\end{array}$ & $\begin{array}{l}\text { It is important } \\
\text { because they } \\
\text { understand it } \\
\text { best }\end{array}$ \\
\hline 36. & $\begin{array}{l}\text { If not, why } \\
\text { not? }\end{array}$ & & & & \\
\hline 37. & $\begin{array}{l}\text { Do you } \\
\text { recommend } \\
\text { any changes in } \\
\text { the way } \\
\text { information } \\
\text { about bed nets } \\
\text { is } \\
\text { communicated? }\end{array}$ & & & & $\begin{array}{l}\text { It's alright; } \\
\text { they explain it } \\
\text { better a } \\
\text { understand it }\end{array}$ \\
\hline
\end{tabular}




\begin{tabular}{|c|c|c|c|c|c|}
\hline \# & Question & $\begin{array}{c}\text { Participant } \\
\# 73\end{array}$ & $\begin{array}{c}\text { Participant } \\
\# 74\end{array}$ & $\begin{array}{c}\text { Participant } \\
\text { \#75 }\end{array}$ & $\begin{array}{c}\text { Participant } \\
\text { \#76 }\end{array}$ \\
\hline 1. & $\begin{array}{l}\text { Are you } 18 \text { or } \\
\text { older? }\end{array}$ & Older female & older male & older male & older male \\
\hline 2. & $\begin{array}{l}\text { If so, are you } \\
\text { between ages } \\
18-29,30-49 \text {, } \\
50-59 \text {, or } 60 \\
\text { and over? }\end{array}$ & $50-59$ & $30-49$ & $50-69$ & $30-49$ \\
\hline 3. & $\begin{array}{l}\text { Are you } \\
\text { traditional } \\
\text { leader/elder, } \\
\text { mother, young } \\
\text { adult (male or } \\
\text { female), or } \\
\text { head of } \\
\text { household? }\end{array}$ & Hoh/mother & hoh & $\begin{array}{l}\text { pastor and head } \\
\text { of household }\end{array}$ & hoh and pastor \\
\hline 4. & $\begin{array}{l}\text { Do you live in } \\
\text { this city, } \\
\text { village, or } \\
\text { community? }\end{array}$ & $\begin{array}{l}\text { Dark forest } \\
\text { field }\end{array}$ & $\begin{array}{l}\text { dark forest } \\
\text { field }\end{array}$ & $\begin{array}{l}\text { dark forest } \\
\text { field }\end{array}$ & $\begin{array}{l}\text { dark forest } \\
\text { field }\end{array}$ \\
\hline 5. & $\begin{array}{l}\text { If yes, how } \\
\text { long have you } \\
\text { lived in this } \\
\text { city, village, or } \\
\text { community? }\end{array}$ & 32 yrs. & 6 yrs. & 7 yrs. & 10 \\
\hline 6. & $\begin{array}{l}\text { Which ethnic } \\
\text { group do you } \\
\text { belong to? }\end{array}$ & Bassa & Bassa & Bassa & Bassa \\
\hline 7. & $\begin{array}{l}\text { Which } \\
\text { language(s) do } \\
\text { you speak? }\end{array}$ & Bassa & $\begin{array}{l}\text { Bassa and } \\
\text { English }\end{array}$ & Bassa & $\begin{array}{l}\text { Bassa and } \\
\text { English }\end{array}$ \\
\hline 8. & $\begin{array}{l}\text { Which religion } \\
\text { do you belong } \\
\text { to? }\end{array}$ & Christian & Christian & Christian & Christian \\
\hline 9. & $\begin{array}{l}\text { Have you ever } \\
\text { heard of } \\
\text { malaria? }\end{array}$ & yes & yes & yes & yes \\
\hline 10. & $\begin{array}{l}\text { What is the } \\
\text { name for } \\
\text { malaria in your } \\
\text { local language? }\end{array}$ & Sun-nee & Sun-nee & Sun-nee & $\begin{array}{l}\text { Sun-nee and } \\
\text { jarjar }\end{array}$ \\
\hline 11. & $\begin{array}{l}\text { Have you ever } \\
\text { suffered from } \\
\text { malaria? }\end{array}$ & $\begin{array}{l}\text { Yes, look it is } \\
\text { in me right } \\
\text { now }\end{array}$ & yes & yes & yes \\
\hline 12. & $\begin{array}{l}\text { If no, what } \\
\text { would you do if } \\
\text { you got } \\
\text { malaria? }\end{array}$ & & & & \\
\hline
\end{tabular}




\begin{tabular}{|c|c|c|c|c|c|}
\hline 13. & $\begin{array}{l}\text { If yes, what } \\
\text { kinds of } \\
\text { treatments do } \\
\text { take for } \\
\text { malaria? }\end{array}$ & $\begin{array}{l}\text { Jologbo, } \\
\text { sekou toure } \\
\text { leave, } \\
\text { traditional } \\
\text { medicine }\end{array}$ & $\begin{array}{l}\text { both western } \\
\text { and traditional. } \\
\text { Go to hospital } \\
\text { and take } \\
\text { amodiaquine, } \\
\text { take ganagana } \\
\text { in the bush }\end{array}$ & $\begin{array}{l}\text { plum bark, and } \\
\text { other leave. } \\
\text { Mixed. } \\
\text { Traditional and } \\
\text { western }\end{array}$ & $\begin{array}{l}\text { amodiaquine is } \\
\text { my best. Take } \\
\text { traditional } \\
\text { medicine at } \\
\text { times. Use yarn } \\
\text { leave. Boil it } \\
\text { and drink. Let } \\
\text { then five to } 6 \\
\text { months or } 1 \\
\text { year before you } \\
\text { get it }\end{array}$ \\
\hline 14. & $\begin{array}{l}\text { Do you use bed } \\
\text { nets to fight off } \\
\text { malaria? }\end{array}$ & yes & yes & yes & yes \\
\hline 15. & If not, why not? & & & & \\
\hline 16. & $\begin{array}{l}\text { What do you } \\
\text { use to fight off } \\
\text { malaria? }\end{array}$ & & & & \\
\hline 17. & Does it help? & & & & \\
\hline 18. & $\begin{array}{l}\text { How do you } \\
\text { know? }\end{array}$ & & & & \\
\hline 19. & $\begin{array}{l}\text { If yes, how did } \\
\text { you learn about } \\
\text { bed nets (i.e. } \\
\text { radio, word of } \\
\text { mouth, health } \\
\text { clinic)? }\end{array}$ & $\begin{array}{l}\text { Community } \\
\text { health } \\
\text { workers }\end{array}$ & radio & $\begin{array}{l}\text { community } \\
\text { health workers }\end{array}$ & $\begin{array}{l}\text { word of mouth, } \\
\text { health clinic, } \\
\text { and radio }\end{array}$ \\
\hline 20. & $\begin{array}{l}\text { Which malaria } \\
\text { treatment } \\
\text { program did } \\
\text { you get your } \\
\text { bed nets from? }\end{array}$ & Don't know & $\begin{array}{l}\text { government } \\
\text { hospital }\end{array}$ & don't know & $\begin{array}{l}\text { government } \\
\text { hospital }\end{array}$ \\
\hline 21. & $\begin{array}{l}\text { How long have } \\
\text { you used bed } \\
\text { nets? }\end{array}$ & 3 yrs. & 3 yrs. & 4 yrs. & 5 yrs. \\
\hline 22. & $\begin{array}{l}\text { Did someone } \\
\text { teach you how } \\
\text { to use bed nets? }\end{array}$ & $\begin{array}{l}\text { Yes, those } \\
\text { who brought } \\
\text { it }\end{array}$ & yes & $\begin{array}{l}\text { yes, those who } \\
\text { brought it }\end{array}$ & yes \\
\hline 23. & $\begin{array}{l}\text { How many } \\
\text { people in your } \\
\text { household use } \\
\text { bed nets? }\end{array}$ & 5 & 10 & 6 & 7 persons \\
\hline 24. & $\begin{array}{l}\text { How do people } \\
\text { in your tribe or } \\
\text { culture feel } \\
\text { about using bed } \\
\text { nets? }\end{array}$ & $\begin{array}{l}\text { They are } \\
\text { happy } \\
\text { because it can } \\
\text { protect the } \\
\text { mosquito }\end{array}$ & fine & they are happy & fine \\
\hline 25. & $\begin{array}{l}\text { How do you } \\
\text { feel about using } \\
\text { bed nets? }\end{array}$ & alright & I feel fine & $\begin{array}{l}\text { I am also } \\
\text { happy about it }\end{array}$ & fine \\
\hline 26. & $\begin{array}{l}\text { What things do } \\
\text { you consider } \\
\text { health risks in } \\
\text { your tribe or } \\
\text { culture? (i.e. } \\
\text { malaria, TB) }\end{array}$ & $\begin{array}{l}\text { Dirty water, } \\
\text { toilet, and } \\
\text { those bring } \\
\text { about illness }\end{array}$ & $\begin{array}{l}\text { open toilet, dirt } \\
\text { site, and other } \\
\text { things not good } \\
\text { in the } \\
\text { community }\end{array}$ & $\begin{array}{l}\text { open toilets } \\
\text { and wells, and } \\
\text { bathrooms }\end{array}$ & $\begin{array}{l}\text { plastic in toilet } \\
\text { throw around, } \\
\text { open well, and } \\
\text { dumping of dirt } \\
\text { everywhere }\end{array}$ \\
\hline
\end{tabular}




\begin{tabular}{|c|c|c|c|c|c|}
\hline 27. & $\begin{array}{l}\text { How do you } \\
\text { treat health } \\
\text { risks in your } \\
\text { tribe or culture? }\end{array}$ & & $\begin{array}{l}\text { cover open } \\
\text { toilet and clean } \\
\text { dumpsite }\end{array}$ & $\begin{array}{l}\text { word of mouth, } \\
\text { and walk house } \\
\text { to house }\end{array}$ & $\begin{array}{l}\text { advising } \\
\text { people and } \\
\text { cleaning my } \\
\text { community }\end{array}$ \\
\hline 28. & $\begin{array}{l}\text { How do you } \\
\text { communicate } \\
\text { health risk in } \\
\text { your tribe or } \\
\text { culture? }\end{array}$ & $\begin{array}{l}\text { word of } \\
\text { mouth, walk } \\
\text { to them }\end{array}$ & word of mouth & & \\
\hline 29. & $\begin{array}{l}\text { Do you use } \\
\text { radio, } \\
\text { television, } \\
\text { flyer, or drum } \\
\text { to } \\
\text { communicate } \\
\text { health risks? }\end{array}$ & & & & \\
\hline 30. & $\begin{array}{l}\text { Explain some } \\
\text { of the } \\
\text { difficulties in } \\
\text { communicating } \\
\text { health risks in } \\
\text { your tribe or } \\
\text { culture? }\end{array}$ & & & & \\
\hline 31. & $\begin{array}{l}\text { How do the } \\
\text { people who } \\
\text { distribute the } \\
\text { bed nets } \\
\text { communicate } \\
\text { with you? }\end{array}$ & & & & \\
\hline 32. & $\begin{array}{l}\text { Do you feel } \\
\text { you usually get } \\
\text { clear } \\
\text { information on } \\
\text { malaria } \\
\text { treatment and } \\
\text { health risks? }\end{array}$ & & & yes, clear. & yes \\
\hline 33. & $\begin{array}{l}\text { Whether you } \\
\text { answer yes or } \\
\text { no, how do you } \\
\text { think health } \\
\text { risks should be } \\
\text { communicated? }\end{array}$ & & & & $\begin{array}{l}\text { by using the } \\
\text { bed nets and } \\
\text { over radio, and } \\
\text { community } \\
\text { outreach from } \\
\text { house to house. } \\
\text { Use the } \\
\text { language for } \\
\text { each } \\
\text { community }\end{array}$ \\
\hline 34. & $\begin{array}{l}\text { Should your } \\
\text { language, } \\
\text { culture, or } \\
\text { tradition matter } \\
\text { in } \\
\text { communicating } \\
\text { health risks to } \\
\text { you? }\end{array}$ & yes & yes & yes & yes \\
\hline
\end{tabular}




\begin{tabular}{|c|l|l|l|l|l|}
\hline 35. & $\begin{array}{l}\text { If so, why and } \\
\text { in what ways? }\end{array}$ & $\begin{array}{l}\text { because she is } \\
\text { Bassa and that } \\
\text { the Bassa she } \\
\text { can } \\
\text { understand }\end{array}$ & $\begin{array}{l}\text { because it is } \\
\text { our mother } \\
\text { dialect and } \\
\text { where the } \\
\text { people can } \\
\text { understand In } \\
\text { communicating } \\
\text { the health risk } \\
\text { in the } \\
\text { community }\end{array}$ & $\begin{array}{l}\text { my language is } \\
\text { the best. } \\
\text { Because it is } \\
\text { my language I } \\
\text { born in and } \\
\text { understand }\end{array}$ & $\begin{array}{l}\text { because it is } \\
\text { our general } \\
\text { language in } \\
\text { this county } \\
\text { besides the } \\
\text { English }\end{array}$ \\
\hline 36. & $\begin{array}{l}\text { If not, why not? } \\
\text { recommend } \\
\text { any changes in } \\
\text { the way } \\
\text { information } \\
\text { about bed nets } \\
\text { is } \\
\text { communicated? }\end{array}$ & okay & it is okay & $\begin{array}{l}\text { yes I want to } \\
\text { recommend } \\
\text { this. The } \\
\text { mistake they } \\
\text { made is that } \\
\text { they don't } \\
\text { explain if we } \\
\text { should wash it } \\
\text { before use or } \\
\text { still just like } \\
\text { that. Create } \\
\text { doubt after } \\
\text { burning people } \\
\text { skin. Suggest } \\
\text { more education } \\
\text { on usage }\end{array}$ \\
\hline
\end{tabular}




\begin{tabular}{|c|c|c|c|c|c|}
\hline \# & Question & $\begin{array}{c}\text { Participant } \\
\# 77\end{array}$ & $\begin{array}{c}\text { Participant } \\
\text { \#78 }\end{array}$ & $\begin{array}{c}\text { Participant } \\
\text { \#79 }\end{array}$ & $\begin{array}{c}\text { Participant } \\
\text { \#80 }\end{array}$ \\
\hline 1. & $\begin{array}{l}\text { Are you } 18 \text { or } \\
\text { older? }\end{array}$ & older male & older female & older male & older male \\
\hline 2. & $\begin{array}{l}\text { If so, are you } \\
\text { between ages } \\
18-29,30-49 \text {, } \\
50-59 \text {, or } 60 \\
\text { and over? }\end{array}$ & $18-29$ & 1965 & 67 & $18-29$ \\
\hline 3. & $\begin{array}{l}\text { Are you } \\
\text { traditional } \\
\text { leader/elder, } \\
\text { mother, young } \\
\text { adult (male or } \\
\text { female), or } \\
\text { head of } \\
\text { household? }\end{array}$ & $\begin{array}{l}\text { young adult } \\
\text { male }\end{array}$ & $\begin{array}{l}\text { traditional } \\
\text { leader and } \\
\text { hoh }\end{array}$ & hoh & $\begin{array}{l}\text { hoh/ young } \\
\text { adult }\end{array}$ \\
\hline 4. & $\begin{array}{l}\text { Do you live in } \\
\text { this city, } \\
\text { village, or } \\
\text { community? }\end{array}$ & sanwein & sanwein & sanwein & $\begin{array}{l}\text { fairground } \\
\text { junction }\end{array}$ \\
\hline 5. & $\begin{array}{l}\text { If yes, how } \\
\text { long have you } \\
\text { lived in this } \\
\text { city, village, or } \\
\text { community? }\end{array}$ & 3 yrs. & 3 yrs. & $\begin{array}{l}\text { don't know, } \\
\text { but long } \\
\text { time }\end{array}$ & 10 yrs. \\
\hline 6. & $\begin{array}{l}\text { Which ethnic } \\
\text { group do you } \\
\text { belong to? }\end{array}$ & Bassa & $\begin{array}{l}\text { Rivercess } \\
\text { Bassa }\end{array}$ & Bassa & Bassa \\
\hline 7. & $\begin{array}{l}\text { Which } \\
\text { language(s) do } \\
\text { you speak? }\end{array}$ & $\begin{array}{l}\text { Bassa and } \\
\text { English }\end{array}$ & Bassa & Bassa & English only \\
\hline 8. & $\begin{array}{l}\text { Which religion } \\
\text { do you belong } \\
\text { to? }\end{array}$ & Christian & Christian & Christian & Christian \\
\hline 9. & $\begin{array}{l}\text { Have you ever } \\
\text { heard of } \\
\text { malaria? }\end{array}$ & yes & yes & yes & yes \\
\hline 10. & $\begin{array}{l}\text { What is the } \\
\text { name for } \\
\text { malaria in your } \\
\text { local language? }\end{array}$ & Sun-nee & Sun-nee & Sun-nee & Sun-nee \\
\hline 11. & $\begin{array}{l}\text { Have you ever } \\
\text { suffered from } \\
\text { malaria? }\end{array}$ & yes & yes & yes & yes \\
\hline 12. & $\begin{array}{l}\text { If no, what } \\
\text { would you do } \\
\text { if you got } \\
\text { malaria? }\end{array}$ & & & & \\
\hline
\end{tabular}




\begin{tabular}{|c|c|c|c|c|c|}
\hline 13. & $\begin{array}{l}\text { If yes, what } \\
\text { kinds of } \\
\text { treatments do } \\
\text { take for } \\
\text { malaria? }\end{array}$ & $\begin{array}{l}\text { country } \\
\text { medicine. } \\
\text { Sugarcane } \\
\text { dry, } \\
\text { German } \\
\text { plum bark, } \\
\text { reeves } \\
\text { leaves. } \\
\text { Take } \\
\text { western } \\
\text { medicine } \\
\text { once a } \\
\text { while but I } \\
\text { trust in my } \\
\text { country } \\
\text { medicine }\end{array}$ & $\begin{array}{l}\text { traditional } \\
\text { medicine } \\
\text { because the } \\
\text { tablets don’t } \\
\text { work on me } \\
\text { again. Tot in } \\
\text { the mud, } \\
\text { plum, paupau, } \\
\text { Christmas } \\
\text { leave, put } \\
\text { them together } \\
\text { and drive and } \\
\text { once the } \\
\text { stomach runs } \\
\text { she is well. } \\
\text { No I don't } \\
\text { take it } \\
\text { because it } \\
\text { doesn't work } \\
\text { on me }\end{array}$ & $\begin{array}{l}\text { traditional } \\
\text { medicine. I } \\
\text { take it but it } \\
\text { doesn't work } \\
\text { on me }\end{array}$ & $\begin{array}{l}\text { take ganagana. } \\
\text { Sometimes I } \\
\text { take } \\
\text { chlroquinne }\end{array}$ \\
\hline 14. & $\begin{array}{l}\text { Do you use bed } \\
\text { nets to fight off } \\
\text { malaria? }\end{array}$ & no & $\begin{array}{l}\text { yes, because } \\
\text { they give me } \\
\text { to use }\end{array}$ & & yes \\
\hline 15. & $\begin{array}{l}\text { If not, why } \\
\text { not? }\end{array}$ & & & & \\
\hline 16. & $\begin{array}{l}\text { What do you } \\
\text { use to fight off } \\
\text { malaria? }\end{array}$ & $\begin{array}{l}\text { country } \\
\text { medicine }\end{array}$ & & & \\
\hline 17. & Does it help? & yes & & & \\
\hline 18. & $\begin{array}{l}\text { How do you } \\
\text { know? }\end{array}$ & & & & \\
\hline 19. & $\begin{array}{l}\text { If yes, how did } \\
\text { you learn about } \\
\text { bed nets (i.e. } \\
\text { radio, word of } \\
\text { mouth, health } \\
\text { clinic)? }\end{array}$ & & $\begin{array}{l}\text { they come, } \\
\text { ask for our } \\
\text { names, and } \\
\text { ask us to get } \\
\text { in line to } \\
\text { receive } \\
\text { bednet and } \\
\text { hand them up. } \\
\text { We received } \\
\text { and hanged it } \\
\text { up }\end{array}$ & $\begin{array}{l}\text { community } \\
\text { health } \\
\text { workers to } \\
\text { our homes }\end{array}$ & radio \\
\hline 20. & $\begin{array}{l}\text { Which malaria } \\
\text { treatment } \\
\text { program did } \\
\text { you get your } \\
\text { bed nets from? }\end{array}$ & don't know & don’t know & ngo & usaid \\
\hline 21. & $\begin{array}{l}\text { How long have } \\
\text { you used bed } \\
\text { nets? }\end{array}$ & & & & 4 yrs. \\
\hline 22. & $\begin{array}{l}\text { Did someone } \\
\text { teach you how } \\
\text { to use bed } \\
\text { nets? }\end{array}$ & & & $\begin{array}{l}\text { yes, the ngo } \\
\text { set it up }\end{array}$ & yes \\
\hline
\end{tabular}




\begin{tabular}{|c|c|c|c|c|c|}
\hline 23. & $\begin{array}{l}\text { How many } \\
\text { people in your } \\
\text { household use } \\
\text { bed nets? }\end{array}$ & & $\begin{array}{l}3 \text { persons } \\
\text { because we } \\
\text { have } 3 \text { rooms }\end{array}$ & 6 persons & 12 \\
\hline 24. & $\begin{array}{l}\text { How do people } \\
\text { in your tribe or } \\
\text { culture feel } \\
\text { about using bed } \\
\text { nets? }\end{array}$ & $\begin{array}{l}\text { feel } \\
\text { reluctant } \\
\text { because } \\
\text { they } \\
\text { traditional } \\
\text { medicine } \\
\text { can help } \\
\text { them }\end{array}$ & $\begin{array}{l}\text { oh, we are } \\
\text { happy but } \\
\text { mosquito is } \\
\text { not really in } \\
\text { this area, } \\
\text { except where } \\
\text { storm comes }\end{array}$ & $\begin{array}{l}\text { they are } \\
\text { happy about } \\
\text { it }\end{array}$ & $\begin{array}{l}\text { feel happy to } \\
\text { use and some } \\
\text { people can use } \\
\text { it because of } \\
\text { the heat. The } \\
\text { other people } \\
\text { put it in the sun }\end{array}$ \\
\hline 25. & $\begin{array}{l}\text { How do you } \\
\text { feel about } \\
\text { using bed nets? }\end{array}$ & $\begin{array}{l}\text { I feel } \\
\text { reluctant } \\
\text { because my } \\
\text { country } \\
\text { medicine } \\
\text { can help me }\end{array}$ & $\begin{array}{l}\text { I think I feel } \\
\text { fine because } \\
\text { if white } \\
\text { people bring } \\
\text { something } \\
\text { you are not } \\
\text { supposed to } \\
\text { dispute it. }\end{array}$ & $\begin{array}{l}\text { I am happy } \\
\text { but the one I } \\
\text { have is now } \\
\text { rotten }\end{array}$ & I feel happy \\
\hline 26. & $\begin{array}{l}\text { What things do } \\
\text { you consider } \\
\text { health risks in } \\
\text { your tribe or } \\
\text { culture? (i.e. } \\
\text { malaria, TB) }\end{array}$ & & $\begin{array}{l}\text { sunnie, dirt, } \\
\text { dirty water, } \\
\text { don' t clean } \\
\text { the } \\
\text { environment }\end{array}$ & & \\
\hline 27. & $\begin{array}{l}\text { How do you } \\
\text { treat health } \\
\text { risks in your } \\
\text { tribe or } \\
\text { culture? }\end{array}$ & & $\begin{array}{l}\text { clean the } \\
\text { environment }\end{array}$ & & \\
\hline 28. & $\begin{array}{l}\text { How do you } \\
\text { communicate } \\
\text { health risk in } \\
\text { your tribe or } \\
\text { culture? }\end{array}$ & $\begin{array}{l}\text { use } \\
\text { towncrier }\end{array}$ & $\begin{array}{l}\text { word of } \\
\text { mouth }\end{array}$ & $\begin{array}{l}\text { word of } \\
\text { mouth }\end{array}$ & towncrier \\
\hline 29. & $\begin{array}{l}\text { Do you use } \\
\text { radio, } \\
\text { television, } \\
\text { flyer, or drum } \\
\text { to } \\
\text { communicate } \\
\text { health risks? }\end{array}$ & & & & \\
\hline 30. & $\begin{array}{l}\text { Explain some } \\
\text { of the } \\
\text { difficulties in } \\
\text { communicating } \\
\text { health risks in } \\
\text { your tribe or } \\
\text { culture? }\end{array}$ & & & & \\
\hline 31. & $\begin{array}{l}\text { How do the } \\
\text { people who } \\
\text { distribute the } \\
\text { bed nets } \\
\text { communicate } \\
\text { with you? }\end{array}$ & & & & \\
\hline
\end{tabular}




\begin{tabular}{|c|c|c|c|c|c|}
\hline 32. & $\begin{array}{l}\text { Do you feel } \\
\text { you usually get } \\
\text { clear } \\
\text { information on } \\
\text { malaria } \\
\text { treatment and } \\
\text { health risks? }\end{array}$ & & clear & & yes \\
\hline 33. & $\begin{array}{l}\text { Whether you } \\
\text { answer yes or } \\
\text { no, how do you } \\
\text { think health } \\
\text { risks should be } \\
\text { communicated? }\end{array}$ & & & & word of mouth \\
\hline 34. & $\begin{array}{l}\text { Should your } \\
\text { language, } \\
\text { culture, or } \\
\text { tradition matter } \\
\text { in } \\
\text { communicating } \\
\text { health risks to } \\
\text { you? }\end{array}$ & yes & yes & yes & yes \\
\hline 35. & $\begin{array}{l}\text { If so, why and } \\
\text { in what ways? }\end{array}$ & $\begin{array}{l}\text { my } \\
\text { language } \\
\text { and culture } \\
\text { important } \\
\text { because we } \\
\text { are the } \\
\text { bassolian } \\
\text { respect our } \\
\text { culture }\end{array}$ & $\begin{array}{l}\text { it's important } \\
\text { to speak in } \\
\text { my dialect; I } \\
\text { am a native } \\
\text { woman }\end{array}$ & $\begin{array}{l}\text { yes, I am } \\
\text { happy } \\
\text { because I } \\
\text { can } \\
\text { understand it } \\
\text { better. } \\
\text { Through } \\
\text { community } \\
\text { outreach }\end{array}$ & $\begin{array}{l}\text { culture is } \\
\text { important } \\
\text { because some } \\
\text { people can 't } \\
\text { understand } \\
\text { English and } \\
\text { when I speak } \\
\text { Bassa they } \\
\text { understand it } \\
\text { better }\end{array}$ \\
\hline 36. & $\begin{array}{l}\text { If not, why } \\
\text { not? }\end{array}$ & & & & \\
\hline 37. & $\begin{array}{l}\text { Do you } \\
\text { recommend } \\
\text { any changes in } \\
\text { the way } \\
\text { information } \\
\text { about bed nets } \\
\text { is } \\
\text { communicated? }\end{array}$ & & $\begin{array}{l}\text { the bednet I } \\
\text { have is old } \\
\text { and I want } \\
\text { new one. }\end{array}$ & & okay \\
\hline
\end{tabular}




\begin{tabular}{|c|c|c|c|c|c|}
\hline$\#$ & Question & $\begin{array}{c}\text { Participant } \\
\# 81\end{array}$ & $\begin{array}{c}\text { Participant } \\
\# 82\end{array}$ & $\begin{array}{c}\text { Participant } \\
\# 83\end{array}$ & $\begin{array}{c}\text { Participant } \\
\# 84\end{array}$ \\
\hline 1. & $\begin{array}{l}\text { Are you } 18 \text { or } \\
\text { older? }\end{array}$ & older male & older female & older male & older male \\
\hline 2. & $\begin{array}{l}\text { If so, are you } \\
\text { between ages } \\
18-29,30-49 \text {, } \\
50-59 \text {, or } 60 \\
\text { and over? }\end{array}$ & $50-59$ & $30-49$ & 62 & $18-29$ \\
\hline 3. & $\begin{array}{l}\text { Are you } \\
\text { traditional } \\
\text { leader/elder, } \\
\text { mother, young } \\
\text { adult (male or } \\
\text { female), or } \\
\text { head of } \\
\text { household? }\end{array}$ & hoh & $\begin{array}{l}\text { traditional } \\
\text { leader/mother }\end{array}$ & $\begin{array}{l}\text { hoh/traditional } \\
\text { leader }\end{array}$ & $\begin{array}{l}\text { young adult } \\
\text { male and } \\
\text { hoh }\end{array}$ \\
\hline 4. & $\begin{array}{l}\text { Do you live in } \\
\text { this city, } \\
\text { village, or } \\
\text { community? }\end{array}$ & sanwein & sanwein & sanwein & sanwein \\
\hline 5. & $\begin{array}{l}\text { If yes, how } \\
\text { long have you } \\
\text { lived in this } \\
\text { city, village, or } \\
\text { community? }\end{array}$ & 7 yrs. & born here & born here & 9 yrs. \\
\hline 6. & $\begin{array}{l}\text { Which ethnic } \\
\text { group do you } \\
\text { belong to? }\end{array}$ & Bassa & Bassa & Kru & Bassa \\
\hline 7. & $\begin{array}{l}\text { Which } \\
\text { language(s) do } \\
\text { you speak? }\end{array}$ & Bassa & Bassa & Kru and Bassa & Bassa \\
\hline 8. & $\begin{array}{l}\text { Which religion } \\
\text { do you belong } \\
\text { to? }\end{array}$ & Christian & Christian & Christian & Christian \\
\hline 9. & $\begin{array}{l}\text { Have you ever } \\
\text { heard of } \\
\text { malaria? }\end{array}$ & yes & $\begin{array}{l}\text { yes, they give } \\
\text { it to us }\end{array}$ & yes & yes \\
\hline 10 & $\begin{array}{l}\text { What is the } \\
\text { name for } \\
\text { malaria in your } \\
\text { local language? }\end{array}$ & Sun-nee & Sun-nee & Sun-nee & Sun-nee \\
\hline 11 & $\begin{array}{l}\text { Have you ever } \\
\text { suffered from } \\
\text { malaria? }\end{array}$ & yes & yes & yes & yes \\
\hline 12 & $\begin{array}{l}\text { If no, what } \\
\text { would you do } \\
\text { if you got } \\
\text { malaria? }\end{array}$ & & & & \\
\hline 13 & $\begin{array}{l}\text { If yes, what } \\
\text { kinds of } \\
\text { treatments do } \\
\text { take for } \\
\text { malaria? }\end{array}$ & $\begin{array}{l}\text { traditional } \\
\text { medicine. } \\
\text { Yes, but it } \\
\text { doesn't do } \\
\text { anything }\end{array}$ & $\begin{array}{l}\text { use western } \\
\text { medicine and } \\
\text { got tired, so I } \\
\text { used } \\
\text { traditional } \\
\text { medicine and } \\
\text { I get better. }\end{array}$ & $\begin{array}{l}\text { traditional } \\
\text { medicine }\end{array}$ & $\begin{array}{l}\text { traditional } \\
\text { medicine. } \\
\text { Take } \\
\text { western } \\
\text { medicine but } \\
\text { it doesn't' } \\
\text { work on me }\end{array}$ \\
\hline
\end{tabular}




\begin{tabular}{|c|c|c|c|c|c|}
\hline 14 & $\begin{array}{l}\text { Do you use bed } \\
\text { nets to fight off } \\
\text { malaria? }\end{array}$ & $\begin{array}{l}\text { yes, they } \\
\text { bring it and } \\
\text { we use }\end{array}$ & $\begin{array}{l}\text { yes, we never } \\
\text { had it but now } \\
\text { we get it so } \\
\text { we are using } \\
\text { it }\end{array}$ & & \\
\hline 15 & $\begin{array}{l}\text { If not, why } \\
\text { not? }\end{array}$ & & & & \\
\hline 16 & $\begin{array}{l}\text { What do you } \\
\text { use to fight off } \\
\text { malaria? }\end{array}$ & & $\begin{array}{l}\text { don't know } \\
\text { but an ngo }\end{array}$ & & yes \\
\hline 17 & Does it help? & & & & \\
\hline 18 & $\begin{array}{l}\text { How do you } \\
\text { know? }\end{array}$ & & & & \\
\hline 19 & $\begin{array}{l}\text { If yes, how did } \\
\text { you learn about } \\
\text { bed nets (i.e. } \\
\text { radio, word of } \\
\text { mouth, health } \\
\text { clinic)? }\end{array}$ & radio & radio & radio & radio \\
\hline 20 & $\begin{array}{l}\text { Which malaria } \\
\text { treatment } \\
\text { program did } \\
\text { you get your } \\
\text { bed nets from? }\end{array}$ & & & don't know & radio only \\
\hline 21 & $\begin{array}{l}\text { How long have } \\
\text { you used bed } \\
\text { nets? }\end{array}$ & 3 yrs. & & 4 yrs. & since 2005 \\
\hline 22 & $\begin{array}{l}\text { Did someone } \\
\text { teach you how } \\
\text { to use bed } \\
\text { nets? }\end{array}$ & & $\begin{array}{l}\text { yes, they } \\
\text { hanged it up } \\
\text { themselves }\end{array}$ & & $\begin{array}{l}\text { yes, those } \\
\text { who gave it } \\
\text { to me }\end{array}$ \\
\hline 23 & $\begin{array}{l}\text { How many } \\
\text { people in your } \\
\text { household use } \\
\text { bed nets? }\end{array}$ & 5 persons & 4 persons & 6 persons & 2 persons \\
\hline 24 & $\begin{array}{l}\text { How do people } \\
\text { in your tribe or } \\
\text { culture feel } \\
\text { about using bed } \\
\text { nets? }\end{array}$ & $\begin{array}{l}\text { they are } \\
\text { happy } \\
\text { because when } \\
\text { white people } \\
\text { say use it, } \\
\text { should we } \\
\text { have another } \\
\text { view of it? }\end{array}$ & they feel fine & $\begin{array}{l}\text { they are happy } \\
\text { and they said } \\
\text { it is fine }\end{array}$ & very fine \\
\hline 25 & $\begin{array}{l}\text { How do you } \\
\text { feel about } \\
\text { using bed nets? }\end{array}$ & $\begin{array}{l}\text { I feel very } \\
\text { fine because I } \\
\text { can sleep } \\
\text { sound if I } \\
\text { sleep under it }\end{array}$ & $\begin{array}{l}\text { myself, I am } \\
\text { happy } \\
\text { because } \\
\text { mosquito is } \\
\text { no longer } \\
\text { biting me }\end{array}$ & $\begin{array}{l}\text { I believe it is } \\
\text { good }\end{array}$ & fine for me \\
\hline 26 & $\begin{array}{l}\text { What things do } \\
\text { you consider } \\
\text { health risks in } \\
\text { your tribe or } \\
\text { culture? (i.e. } \\
\text { malaria, TB) }\end{array}$ & & & & \\
\hline
\end{tabular}




\begin{tabular}{|c|c|c|c|c|c|}
\hline 27 & $\begin{array}{l}\text { How do you } \\
\text { treat health } \\
\text { risks in your } \\
\text { tribe or } \\
\text { culture? }\end{array}$ & & & & \\
\hline 28 & $\begin{array}{l}\text { How do you } \\
\text { communicate } \\
\text { health risk in } \\
\text { your tribe or } \\
\text { culture? }\end{array}$ & $\begin{array}{l}\text { word of } \\
\text { mouth }\end{array}$ & $\begin{array}{l}\text { word of } \\
\text { mouth }\end{array}$ & $\begin{array}{l}\text { word of } \\
\text { mouth }\end{array}$ & $\begin{array}{l}\text { word of } \\
\text { mouth }\end{array}$ \\
\hline 29 & $\begin{array}{l}\text { Do you use } \\
\text { radio, } \\
\text { television, } \\
\text { flyer, or drum } \\
\text { to } \\
\text { communicate } \\
\text { health risks? }\end{array}$ & & & & \\
\hline 30 & $\begin{array}{l}\text { Explain some } \\
\text { of the } \\
\text { difficulties in } \\
\text { communicating } \\
\text { health risks in } \\
\text { your tribe or } \\
\text { culture? }\end{array}$ & & & & \\
\hline 31 & $\begin{array}{l}\text { How do the } \\
\text { people who } \\
\text { distribute the } \\
\text { bed nets } \\
\text { communicate } \\
\text { with you? }\end{array}$ & & & & \\
\hline 32 & $\begin{array}{l}\text { Do you feel } \\
\text { you usually get } \\
\text { clear } \\
\text { information on } \\
\text { malaria } \\
\text { treatment and } \\
\text { health risks? }\end{array}$ & & & & \\
\hline 33 & $\begin{array}{l}\text { Whether you } \\
\text { answer yes or } \\
\text { no, how do you } \\
\text { think health } \\
\text { risks should be } \\
\text { communicated? }\end{array}$ & & & & \\
\hline 34 & $\begin{array}{l}\text { Should your } \\
\text { language, } \\
\text { culture, or } \\
\text { tradition matter } \\
\text { in } \\
\text { communicating } \\
\text { health risks to } \\
\text { you? }\end{array}$ & yes & yes & yes & yes \\
\hline
\end{tabular}




\begin{tabular}{|c|c|c|c|c|c|}
\hline 35 & $\begin{array}{l}\text { If so, why and } \\
\text { in what ways? }\end{array}$ & $\begin{array}{l}\text { it is very } \\
\text { important in } \\
\text { my own } \\
\text { language. } \\
\text { Because it is } \\
\text { my dialect } \\
\text { and I can } \\
\text { understand it } \\
\text { well }\end{array}$ & $\begin{array}{l}\text { when they say } \\
\text { something in } \\
\text { our dialect } \\
\text { than we can } \\
\text { understand it } \\
\text { clear without } \\
\text { any doubt }\end{array}$ & $\begin{array}{l}\text { I am happy } \\
\text { when they } \\
\text { speak my } \\
\text { dialect. }\end{array}$ & fine \\
\hline 36 & $\begin{array}{l}\text { If not, why } \\
\text { not? }\end{array}$ & & & & \\
\hline 37 & $\begin{array}{l}\text { Do you } \\
\text { recommend } \\
\text { any changes in } \\
\text { the way } \\
\text { information } \\
\text { about bed nets } \\
\text { is } \\
\text { communicated? }\end{array}$ & & & & $\begin{array}{l}\text { change but } \\
\text { no reason. } \\
\text { The bednet I } \\
\text { have has } \\
\text { holes in it } \\
\text { and } \\
\text { mosquito } \\
\text { can } \\
\text { sometimes } \\
\text { penetrate it }\end{array}$ \\
\hline
\end{tabular}

